\title{
Curvotaxis \\ and \\ Pattern Formation in the Actin \\ Cortex of Motile Cells
}

\author{
Dissertation \\ for the award of the degree "Doctor rerum naturalium" \\ of the Georg-August-Universität Göttingen \\ within the doctoral program \\ Physics of Biological and Complex Systems \\ of the Georg-August University School of Science \\ (GAUSS)
}

submitted by
Christoph Blum
from Suhl
Göttingen, 2015 


\section{Thesis Committee:}

Prof. Dr. Eberhard Bodenschatz

Abteilung Hydrodynamik, Strukturbildung und Biokomplexität, Max-Planck-Institut für Dynamik und Selbstorganisation

Prof. Dr. Jörg Enderlein

Drittes Physikalisches Institut, Georg-August-Universität Göttingen

Prof. Dr. Annette Zippelius

Institut für Theoretische Physik, Georg-August-Universität Göttingen

\section{Members of the Examination Board:}

Referee: Prof. Dr. Eberhard Bodenschatz

Abteilung Hydrodynamik, Strukturbildung und Biokomplexität, Max-Planck-Institut für Dynamik and Selbstorganisation

2nd Referee: Prof. Dr. Jörg Enderlein

Drittes Physikalisches Institut, Georg-August-Universität Göttingen

\section{Further Members of the Examination Board:}

\section{Prof. Dr. Annette Zippelius}

Institut für Theoretische Physik, Georg-August-Universität Göttingen

Dr. Marco Tarantola

Abteilung Hydrodynamik, Strukturbildung und Biokomplexität, Max-Planck-Institut für Dynamik und Selbstorganisation

\section{Prof. Dr. Stephan Herminghaus}

Abteilung Dynamik komplexer Fluide,

Max-Planck-Institut für Dynamik and Selbstorganisation

\section{Dr. Michael Wilczek}

Max-Planck Forschungsgruppe "Theorie turbulenter Strömungen", Max-Planck-Institut für Dynamik and Selbstorganisation

Date of oral examination: September 16, 2015 
“I have a friend who's an artist and has sometimes taken a view which I don't agree with very well. He'll hold up a flower and say "look how beautiful it is," and I'll agree. Then he says "I as an artist can see how beautiful this is but you as a scientist take this all apart and it becomes a dull thing," and I think that he's kind of nutty. First of all, the beauty that he sees is available to other people and to me too, I believe. Although I may not be quite as refined aesthetically as he is ... I can appreciate the beauty of a flower. At the same time, I see much more about the flower than he sees. I could imagine the cells in there, the complicated actions inside, which also have a beauty. I mean it's not just beauty at this dimension, at one centimeter; there's also beauty at smaller dimensions, the inner structure, also the processes. The fact that the colors in the flower evolved in order to attract insects to pollinate it is interesting; it means that insects can see the color. It adds a question: does this aesthetic sense also exist in the lower forms? Why is it aesthetic? All kinds of interesting questions which the science knowledge only adds to the excitement, the mystery and the awe of a flower. It only adds. I don't understand how it subtracts."

Richard P. Feynman, 1981 



\section{Contents}

\begin{tabular}{lll}
\hline & Introduction & 7
\end{tabular}

2 Model Organism 11

$2.1 \quad$ Dictyostelium discoideum . . . . . . . . . . . . . . . . . . . . 11

2.1 .1 Life Cycle. . . . . . . . . . . . . . . 12

2.2 Cell Migration . . . . . . . . . . . . . . . . . . . . . . 13

2.2 .1 Cytoskeleton . . . . . . . . . . . . . 15

2.2 .2 Migration Mechanism . . . . . . . . . . . . 20

2.3 Chemotaxis . . . . . . . . . . . . . . . 25

2.3 .1 Chemotactic Signaling Network . . . . . . . . . . 25

2.3 .2 Chemotactic Migration . . . . . . . . . . . . . . . 29

2.3 .3 Genetic Mutations . . . . . . . . . . . . . 32

2.4 Theoretical Descriptions . . . . . . . . . . . . . . 33

2.4 .1 Activated Membrane Patches . . . . . . . . . . . . . . 33

2.4 .2 Statistical Geometrical Theories . . . . . . . . . . . . . 35

\begin{tabular}{lll}
\hline 3 & Experimental Setup and Methods & 37
\end{tabular}

3.1 Cell Culture and Genetics . . . . . . . . . . . . . . . . . 37

3.2 Methods . . . . . . . . . . . . . . . . . . . . . 40

3.2 .1 Microfluidics . . . . . . . . . . . . . . . . . 40

3.2 .2 Microscopy . . . . . . . . . . . . . . . . . 42 
3.2 .3 Experimental Setups $\ldots \ldots \ldots \ldots$. . . . . . . . . . 47

4 Curvotaxis 53

4.1 Related research. . . . . . . . . . . . . . . . . . . . . . 54

4.2 Curvature Definition and Analysis Techniques . . . . . . . . . 57

4.2 .1 Two-Dimensional Line Curvature . . . . . . . . . . . . 57

4.2 .2 Three-Dimensional Curvature . . . . . . . . . . . . 58

4.2 .3 Projections and Lengths $\ldots \ldots \ldots$

$4.2 .4 \quad$ Vector Decomposition . . . . . . . . . . . . . . . . . . 64

$4.2 .5 \quad$ Curvotactic Anisotropy Parameter . . . . . . . . . . . . 65

$4.2 .6 \quad$ PCA-Characterization of Trajectories with Convex Hulls . . 67

4.2 .7 Atomic Force Microscopy for Substrate Analysis . . . . . . 69

4.3 Curvotaxis on Cylinders $\ldots \ldots \ldots \ldots$

$4.3 .1 \quad$ Representative Single Experiment . . . . . . . . . . . 73

4.3 .2 Influence of the Experimental Setup . . . . . . . . . 76

4.3 .3 Curvature-Dependence of Curvotaxis $\ldots \ldots \ldots . . .484$

4.3 .4 Biochemical Origins of Curvotaxis . . . . . . . . . 85

4.4 Curvotaxis on Wrinkled Substrates . . . . . . . . . . . . . . . . 87

4.4 .1 Exemplary Curvotaxis Result on Wrinkles. . . . . . . . . 88

4.4.2 Dependence of Curvotaxis on Maximal Radius of Curvature of Wrinkles . . . . . . . . . . . . . . . . 993

4.5 Discussion: Cylinders vs. Wrinkles $\ldots \ldots \ldots \ldots$. . . . . . 97

5 Pattern Formation in the Actin Cortex 103

5.1 Related research. . . . . . . . . . . . . . . . . . . . . 104

5.2 Analysis Methods . . . . . . . . . . . . . . . . 106

5.2 .1 Active Contour Cell Outline Detection . . . . . . . . . . 106

5.2 .2 Spatiotemporal Cross-Correlation . . . . . . . . . . 110

5.3 Results of Confocal Laser Scanning Microscopy. . . . . . . . . . 112

5.3 .1 Results . . . . . . . . . . . . . . . . . . . . . 112

5.4 Fast Spinning Disc Confocal Laser Scanning Microscopy . . . . . 128

$5.4 .1 \quad$ Results for Wildtype Cells $\ldots \ldots \ldots \ldots$

$5.4 .2 \quad$ PTEN Knockout Cells . . . . . . . . . . . . . . . 138 
5.4 .3 Myosin-II Knockout Cells . . . . . . . . . . . . . . . . . 144

5.5 Discussion . . . . . . . . . . . . . . . . . . . . . . . . . . . . 148

6 Conclusion and Outlook 153

6.1 Curvotaxis . . . . . . . . . . . . . . . . . . . . . . . 153

6.2 Pattern Formation in the Actin Cortex . . . . . . . . . . . 154

6.3 Investigation of Signaling Dynamics in Curvotactic Migration . . 155

6.4 Outlook . . . . . . . . . . . . . . . . . . . . . . . . . 157

\begin{tabular}{ll}
\hline Bibliography & 159
\end{tabular}

List of Figures 172

\begin{tabular}{ll}
\hline Acknowledgments & 179
\end{tabular}

$\begin{array}{ll}\text { Curriculum Vitae } & 183\end{array}$ 
Contents 


\section{CHAPTER 1}

\section{Introduction}

In nature, spatiotemporal dynamics represent life. A world in equilibrium would be a dull and uninteresting world. That life is an out of equilibrium system was already discussed by the ancient greek philosophers Heraclitus and Plato in their

statement "Panta rhei' ${ }^{1}$ which means "Everything flows". One aspect of living dynamics is cell migration, which is known to be very similar in many different organisms. For instance, the amoeboid cell migration describes a type of cell motility that is conserved from amoeba to human immune cells. Neutrophil granulocytes, a kind of phagocytes of the mammalian immune system, migrate from blood vessels through tissue towards spots of inflammation. To find this inflammation, they use a chemical "compass" that contains a system to receive and process a chemical signal that guides the neutrophil to the inflammation by leading it towards higher concentration of a chemoattractant molecule. This process is known as chemotaxis. Like amoeboid cell migration, chemotaxis is a process found often and is widespread

\footnotetext{
${ }^{1}$ Idea of Heraclitus, used by Plato, wordly by Simplikios
} 
in nature. A well-known but unpleasant process where chemotaxis plays a major role is cancer metastasis. The social amoeba Dictyostelium discoideum (D.d.) is a commonly used model organism to study migration as well as chemotaxis. It will be the organism with which we study cell migration and chemotaxis in this thesis.

The amoeba $D . d$. has three different life cycles. There is a vegetative cycle, in which the amoebae live as individuals in the forest soil and prey on bacteria. As long as the amount of food is sufficient, the cells will grow and divide by mitosis into two genetically identical daughter cells. Depending on the environmental conditions, the cells will develop further either in the sexual cycle, in which $D$. $d$. cells are able to mate, or in the social cycle, where the cells are able to synchronize with each other and finally form a multicellular organism. $D . d$. cells enter this cycle if they are deprived of nutrients. Therefore, they express a completely novel machinery that was not needed before the starvation. As receptors for the chemoattractant cAMP (cyclic adenosine monophoshate), the machinery for signal transduction of this chemoattractant signal and the tools for secreting cAMP on their own are needed. Using these newly developed tools, the cells synchronize with each other, come together and form a multicellular organism composed of a large number of differentiated single cells. This multicellular organism has the evolutionary advantages of a faster movement and the exploration of larger surrounding area to find new food sources. In Figure 2.1, the social cycle can be seen in the lower left. In the beginning, single amoeboid cells hunt bacteria in the soil. For the transition between the vegetative cycle and the social cycle, the amount of bacterial prey is crucial. If the cells start to starve, the development of the cells begins. 
The natural environment for cell migration, whether these are immune cells, cancer cells or $D . d$., is a very complex geometry such as cylindrical blood vessels, living tissue or forest soil and leaf litter. Hence it is reasonable to assume that amoeboid migration is dependent on the properties of the surrounding geometry. Due to challenges of the experimental setups, most investigations of cell migration are still performed in two-dimensional setups, as for instance a microfluidic channel with glass slide at the bottom. The first part of this thesis is to investigate cell migration of $D . d$. on curved substrates. We developed two setups to examine the effect of curved substrates to amoeboid cell migration of $D$. $d$. As we were able to find directed the cell migration along high curvature, we called this effect "Curvotaxis".

A very interesting concept in modern science is pattern formation, which is also referred to as self-organization. The fact that nature produces structures and patterns puzzles scientists and philosophers since ancient greece. Modern philosophy is also concerned about self-organization as can be seen in Immanuel Kant's work from 1790 entitled "Kritik der Urteilskraft" about the aesthetics of patterns in nature. In terms of physics and computer science, the leading figure was Alan Turing with his groundbreaking 1952 paper "The Chemical Basis of Morphogenesis", in which he introduced a mathematical model that could explain pattern formation processes (78).

A special process of pattern formation is the directional solidification. It was found by Utter and colleagues $(\underline{81})$ that in directional solidification alternating tip splitting occurs, which is quite similar to the splitting of pseudopodia in $D$. $d$. cell migration. In directional solidification the reason of the split is a linear instability of the curved front. This leads to the question of the underlying mechanism of the splitting pseudopodia in D. $d$. 
In chapter five of this thesis, we want to study the process of $D . d$. cell migration, considering the geometrical shape-changes of single cells. We investigate the correlations of cell signaling dynamics, actin polymerization and cell geometry. The reason to study this problem is to confirm the cause-and-effect relation of the already known parts of the signaling cascades and the cell membrane curvature. We address the question of whether membrane curvature is responsible for a higher possibility for a pseudopod to start and hence to activate the signaling cascade.

The thesis starts with an introduction to the organism $D . d$. and the basis of chemotactic cell migration. Thereafter the materials and methods used for the work will be presented. Chapter 4 contains the results of the "Curvotaxis" chapter. The correlation of the signaling dynamics with the cell geometry is presented in Chapter 5. The work is concluded with a conclusion and an outlook. 


\section{CHAPTER 2}

\section{Model Organism}

\subsection{Dictyostelium discoideum}

The social amoeba $D . d$. is one of the most extensively studied model organisms for cell motility, amoeboid motion and chemotaxis. Despite its early evolution (it branched off the phylogenetic tree between plants and fungi) it shares many biochemical aspects with younger eukaryotic organisms, for example its dependence on the messenger cyclic adenosine monophosphate (cAMP). D. $d$. is a haploid organism with a fully sequenced genome (29) and therefore genetic modifications are comparatively easy. The ability to modify the organism allows for investigation of the biochemical networks and signaling cascades. The organism $D . d$. is studied due to the strong similarities in migration to human neutrophils or cancer cells, and also from a developmental biophysics point of view: its life cycle possesses three different parts, particularly the so-called social cycle is a fascinating mechanism 
rarely occurring in nature. If a single soil-living amoeba ceases to find sufficient food supplies, mainly bacteria, they start to synchronize with each other by emitting the signaling molecule cAMP. This cAMP activates a signaling pathway leading to chemotactic migration, i.e. directed motility in the direction of the maximal chemoattractant gradient. Additionally, cells that sense cAMP are also emitting cAMP and in this way all cell will enter a synchronized stage, where cAMP waves can travel through the complete population.

\subsubsection{Life Cycle}

$D . d$. possesses a life cycle with three different parts that fulfill different purposes, as shown schematically in Figure 2.1. In the vegetative cycle, $D$. $d$. cells face no food deprivation and therefore the cells can grow and divide by mitosis, leading to cell doubling every 8-9 hours and under best environmental conditions it can decrease to 4 hours (44).

Additionally, $D . d$. cells have the possibility to proliferate in a sexual cycle allowing gene exchange between individuals. This only occurs under special environmental conditions, including darkness, wet conditions, low phosphate ion concentrations and the presence of calcium ions (57), leading to a macrocyst (44, 74).

The social cycle is most relevant for this thesis as it relies on cAMP-chemotaxis. As soon as the cells are deprived of food, they enter this cycle. They then upregulate several genes that enable them to sense and produce a cAMP. The secreted cAMP forms a gradient towards which surrounding amoebas migrate via chemotaxis. After a streaming stage, where first cell-cell contacts are temporarily formed, the cells aggregate and form an collective organism called mound. The mound can subsequently 
form a so-called finger at its top, which may evolve into a slug-like stage that is able to migrate for some distance. The final goal of this multicellular organism, built from former individual cells is to form a fruiting body including spore cells, resting on its stalk. These spores can then be transported by wind, water as well as other soil organisms to a place where the cells may find improved living conditions. In this case the spores can disseminate to release single amoebas. Furthermore, $D . d$. exhibit the following fascinating phenomenon: to support the fruiting body some cells have to sacrifice themselves to build the stalk and hence to assure the survival of the colony (20). It was found that approximately $20 \%$ of the cells have to sacrifice themselves, while $80 \%$ of the cells will form spores.

\subsection{Cell Migration}

Migration is one of the classical properties of life. The variety of migrational modes is huge, like for example: swimming of bacteria, crawling of fish keratocytes, floating and rolling of white blood cells before leaving the human blood vessels. Here we will focus on amoeboid cell migration, which we will call cell crawling. Crawling cells need a supporting surface to migrate on. If we allow for a wider definition of the term crawling $D . d$. migration through a very viscous fluid (5) with the same geometrical properties as on a substrate may also be captured.

In the following section we will present the underlying structural network for cell migration, namely the cytoskeleton. Moreover we will discuss the general mechanism of cell migration and details of $D$. $d$. cell migration.

Migration towards environmental cues is a crucial part of $D . d$. motility. This not only includes chemical cues, as in the case of the well-studied chemotaxis. $D$. $d$. is 


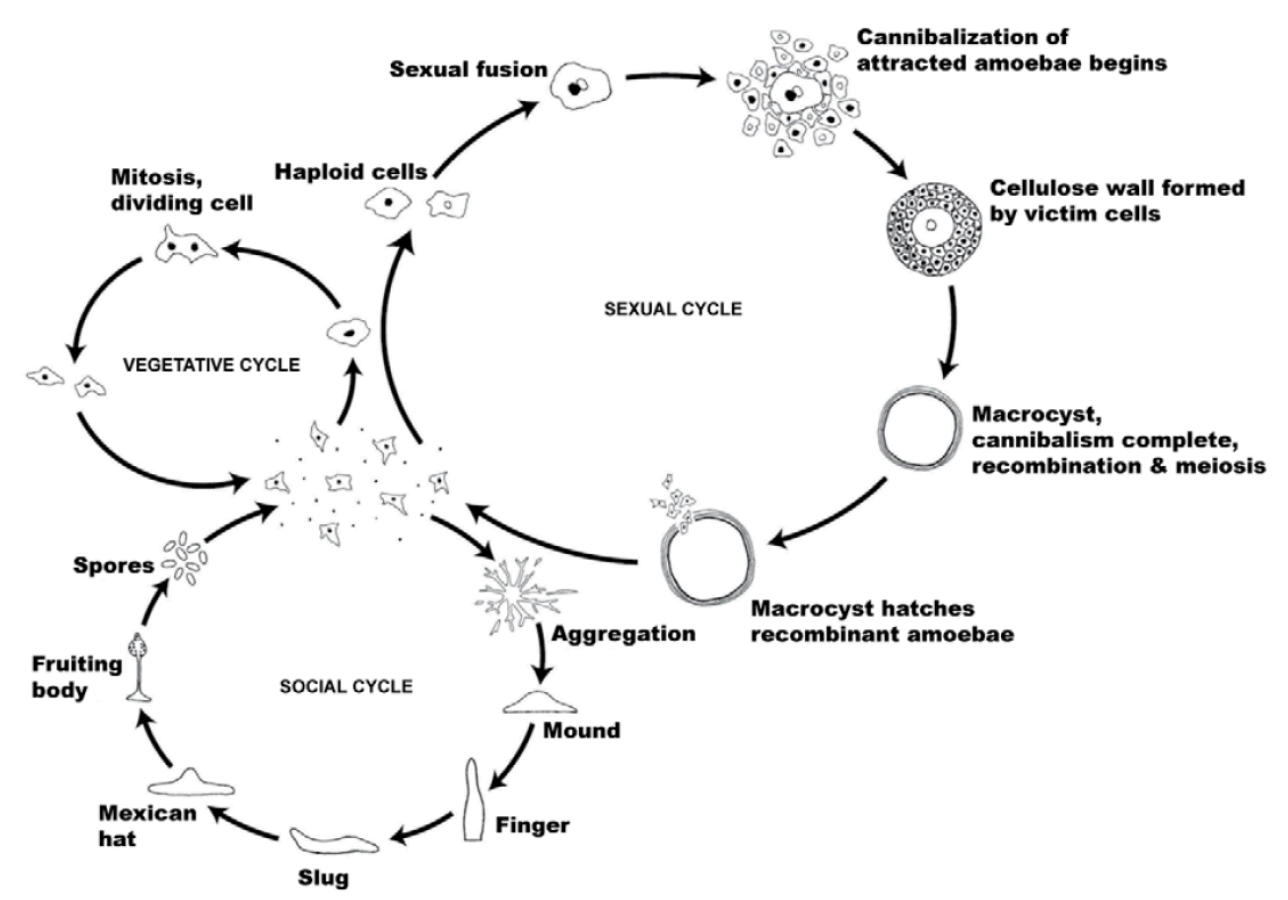

Figure 2.1: Overview of the three parts of the life cycle of $D . d$. In the vegetative cycle, the cells grow and divide by mitosis. In the sexual cycle, two haploid cells fuse and after several intermediate steps the recombinant cells leave the so-called marcocyst. The cycle of most interest in this thesis is the social cycle, where starved amoeba synchronize with each other to aggregate and form a multicellular organism with stalk and spore cells. Scheme taken from (33), Copyright 2010, D. Brown and J.E. Strassmann. Permission granted by the Creative Commons Attribution.

known to show phototaxis, thermotaxis, acidotaxis, mechanotaxis and electrotaxis. The directed migration is found either on the slug like stage or on the single cell stage:

- Slug stage: Phototaxis is the ability to migrate towards a light source and is observed in $D . d$. during the slug-like stage $(28,54)$. It was also observed that both the slug like stage and the single amoeba show directed migration in 
temperature gradients (thermotaxis) (38). Bonner and colleagues investigated in 1985 the dependence of the slug-like stage on $\mathrm{pH}$ gradients (11) and found that the slugs orient towards the more acidic side. Hence it was termed acidotaxis.

- Single cell stage: Mechanotaxis stands for the ability to migrate in response to mechanical cues. For instance $D . d$. is known to react to sufficiently high shear stress (23). The existence of an electric field has also been observed to influence the cell migration and it is refered to as electrotaxis (68).

Even though these various directional cues of $D . d$. cell migration are known, there is still more aspects of directed migration to be discover. We will present a new mode of guided migration we named curvotaxis in chapter 4

\subsubsection{Cytoskeleton}

The basis of cell morphology and migration is the cytoskeleton. The eukaryotic cytoskeleton consists of three main components, namely actin-filaments, intermediate filaments and microtubuli. These can be associated with motor-proteins, but also binding-proteins that create a stiff but still dynamic and flexible fibrous skeleton that gives the cell structure.

The structure of the cytoskeleton was found to be strongly conserved in most of eukaryotic cells (59). The main stabilizing constituents of the cytoskeleton are actin and myosin that also the building blocks of mammalian muscles, are at the center of the $D . d$. cell cortex, but also many other proteins play a role in the actin cytoskeleton (56). 


\subsubsection{Actin}

Actin molecules can be found in nearly every if not all eukaryotic cells. There is a huge variety in actin molecules. An intriguing fact is that, despite this variety, the actin isoforms are highly conserved, meaning that around $90 \%$ of the amino-acid sequence are identical (58).

Actin exists in the cells in a monomeric form called globular actin (G-actin) as well as in a polymeric, filamentous form which is called F-actin. Actin monomers bind to adenosine triphosphate (ATP), adenoside diphosphate (ADP), as they are the enzymes (pyrophosphatase) substrates and its cofactor and to magnesium cations $\mathrm{Mg}^{2+}$.

The main question regarding actin is how the dynamical change between monomeric G-actin and filamentous F-actin governs the cell morphology. As described in (59, 60, 61), this complex system needs several interacting proteins. Figure 2.2 shows the most important processes, starting with the processes in vitro to describe the process in controlled conditions:

1) The in vitro formation of a actin dimer from two actin monomers is an energetically unfavorable process that is unlikely to happen. Likewise the formation of a trimer from a dimer and a monomer is less likely than the back reaction in which a trimer dissociates. Going from trimers to fibers instead becomes energetically favorable. F-actin filaments are polarized, like their G-actin constituents. As they have an orientation that leads to different rate constants for growing and shrinking of the filaments. The growing end is called the barbed end, whereas the shrinking one is the pointed end. Apart from purified actin, also the presence of phosphate groups e.g. the activation state, thus ATP and ADP play a crucial role. The binding 


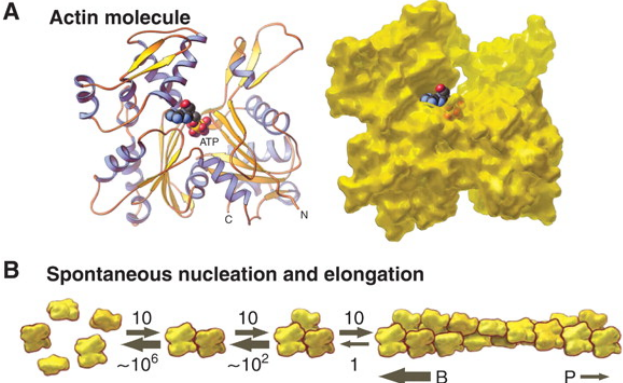

C

Monomer binding

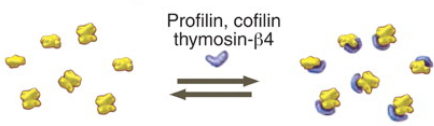

D Nucleation and elongation by formin

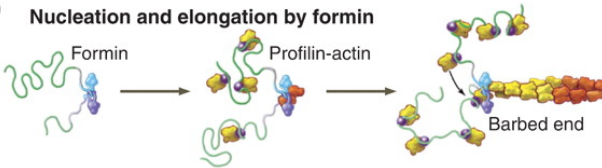

E Branching nucleation by Arp2/3 complex

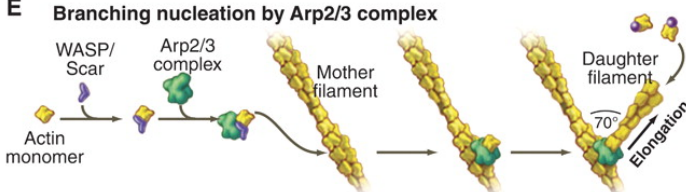

F Reactions of filaments

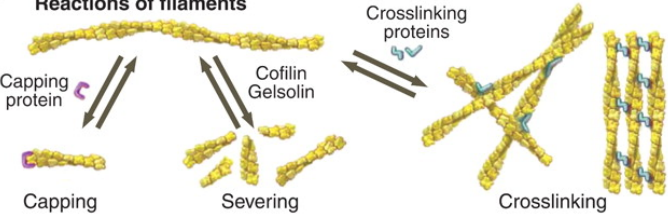

G Myosin-V walking on an actin filament
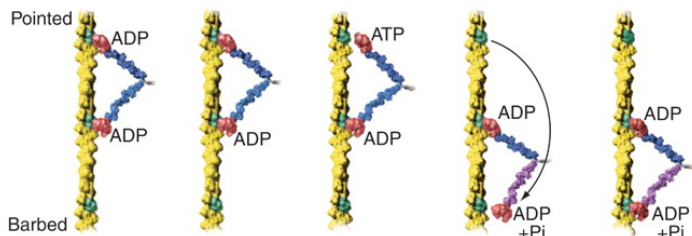

Figure 2.2: Main properties of actin. A shows a single actin monomer as a ribbon as well as space-filling models. In $\mathbf{B}$ the rate constants for spontaneous actin assembly and disassembly are shown. The effect of actin nucleation and elongation factors are shown in $\mathbf{C}$ and $\mathbf{D}$, namely formin, profilin \& cofilin. $\mathbf{E}$ describes how the proteins WASP and Scar activate the Arp2/3 complex that cause a branching of an actin filament. F Several possible ways how an actin filament can develop are shown. It can be capped, which leads to a stop of polymerization at the barbed end. It can be severed into smaller pieces and finally back into monomers, and can be cross-linked. G shows a myosin-V motor walking on an actin filament using ATP. Image taken from (61), Reprinted with permission from AAAS.

of ATP-actin has a different rate constant than the binding of ADP-actin. ATP-actin will be hydrolyzed to ADP and phosphate. This process has a half time of around 2 seconds $(\mathbf{5 8})$. The phosphate may now reversibly dissociate with a half time of 350 seconds. In the described orientation of the filament, the phosphate release is more probable at the pointed end. In steady state conditions this leads to a process which is known as treadmilling, where the length of the filament and hence the amount of actin molecules stays constant via adding molecules to the barbed end and releasing 
molecules from the pointed end, but during this the actin filament is displaced.

2) In vivo the nucleation process is governed by more than 60 protein families which are responsible for binding, capping, cross-linking etc.

One of these proteins is profilin, which binds actin monomers and also catalyzes the exchange of ADP to ATP. Additionally profilin inhibits the growth of the pointed end of the actin filament. This growth of the actin filament also involves formins that initiate the binding of free actin as profilin-actin binds to formin. With that the actin monomers are transfered on the barbed end of actin filaments (61).

$A D F / C o f i l i n$ is a family of proteins that are able to bind ADP-actin monomers and inhibit nucleotide exchange. Hence they play a role in severing ADP-actin filaments.

The influence of the Arp2/3 complex is essential in the formation of the actin cytoskeleton and thus the migration of cells. The Arp $2 / 3$ complex is responsible for the branching of actin filaments, as shown by the sketch in Figure 2.2 F. The complex binds to an existing actin filament and offers a binding site for actin monomers. Hence straight filaments can become a branched network due to the action of the Arp $2 / 3$ complex with an branching angle of $70^{\circ}$.

The SCAR/WAVE protein is responsible for the transduction of the external chemotactic signal that links the cell with the actin cytoskeleton and hence the migration. $S C A R / W A V E$ is the last step of the signaling cascade regulating the actin machinery, due to the activation of the Arp2/3 complex that enhances branching of actin filaments (see Figure 2.3). 


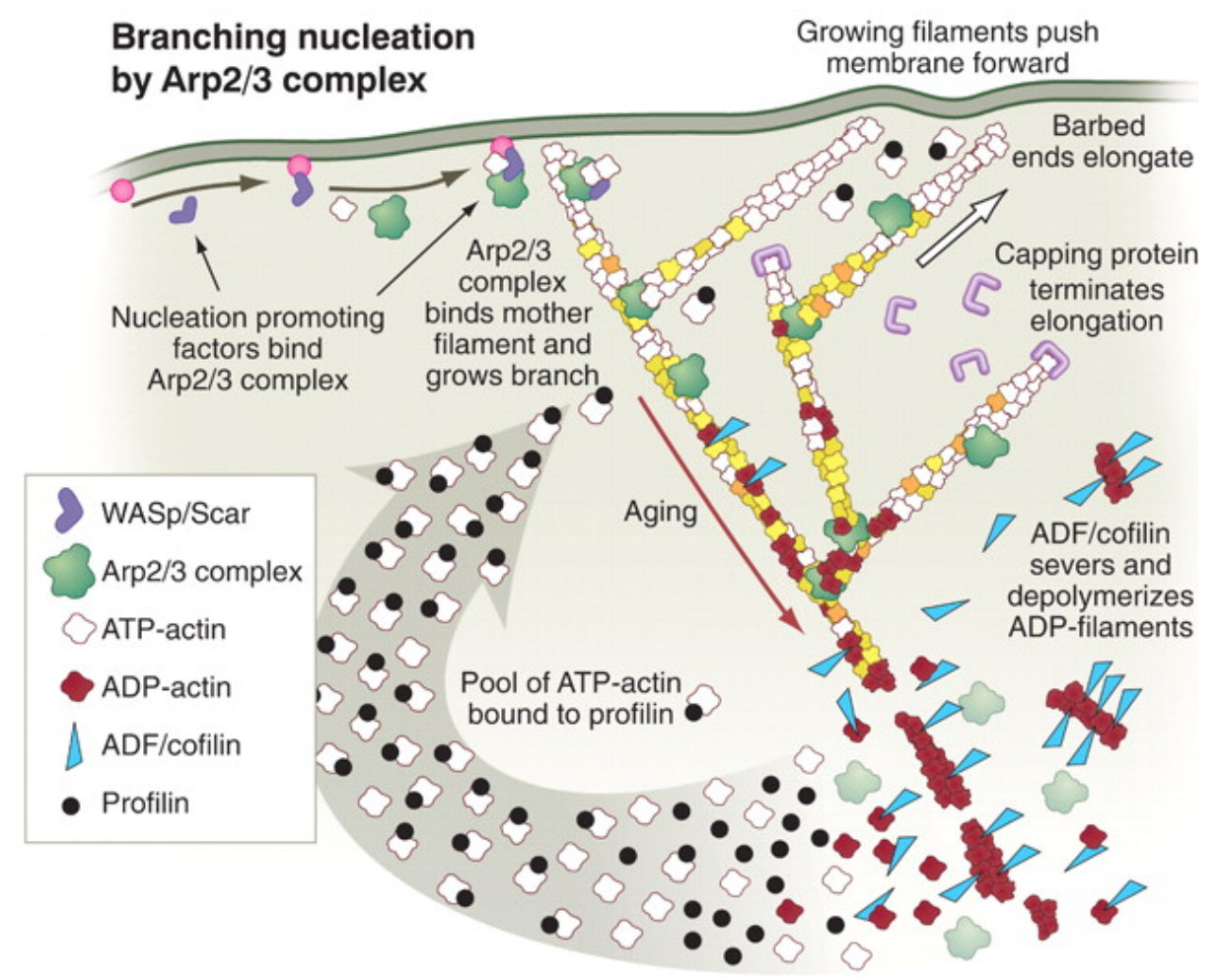

Figure 2.3: Sketch of the actin machinery. The extracellular stimuli are transformed to an intracellular signal and then amplified through a signaling cascade until they activate $W A S P / S C A R$, which activates Arp $2 / 3$ and leads to filament branching. The barbed ends of the filaments grow and push against the cell membrane leading to protrusion. Over time, some filaments are capped and the pointed ends of filaments age, meaning that the actin monomers are mainly bound to ADP. ADF/cofilin binds to ADP-actin and severs and depolymerizes ADP-actin filaments. The resulting ADP-actin monomers bind to profilin which catalyzes the exchange of ADP for ATP and hence allows the actin monomers to be incorporated into freshly polymerized actin-ATP filaments again. Image modified from (61), Reprinted with permission from AAAS.

\subsubsection{Myosin}

The role of myosin motors in the cytoskeletal dynamics is of great importance. The myosin motors can be viewed as the side of the coin opposite of actin in actomyosin 
based motility. As the dynamic processes of actin are necessary to push the plasma membrame forward of the cell membrane, there is a need to control the dynamics of the cell rear, also called uropod. This control is taken care of by myosin motors, especially myosin-II, that are responsible for the contractile forces at the cell back. In the actomyosin cortex of $D$. $d$. cells, this contraction secures the structural integrity of the cell and its pseudopod extensions.

In $D . d$. cytoskeleton myosin-II is the most important form of myosin studied in this thesis, as it is strongly involved in the cytoskeleton (86). It is composed by two essential light chains, two regulatory light chains and two myosin heavy chains (MHC), which itself possess a globular head, see Figure 2.4 .

The importance of myosin-II in the actin cytoskeleton is due to its function of being able to transform chemical energy into mechanical energy or force. In Figure 2.4 (b) the effective process of the myosin-II motor protein is shown. A bundle of two myosin-II proteins can shift actin filaments against each other. The motor domain allows the myosin-II to bind to actin and this actomyosin complex can contract by a hydrolization of ATP (14).

\subsubsection{Migration Mechanism}

Amoeboid migration is widely conserved over a huge variety of species. Its universality is an important aspect and allows us to study this general process with D. $d$.

The mechanism of cell migration consists of four main steps, namely attachment, protrusion, contraction and detachment. Below we will explain these individual steps 


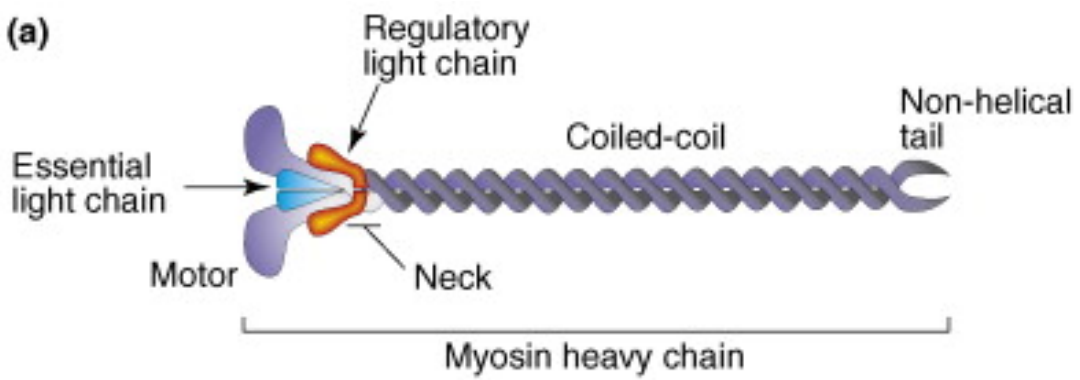

(b)

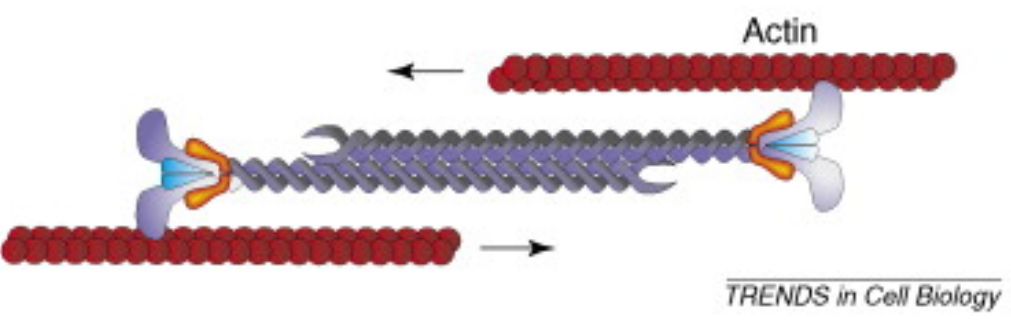

Figure 2.4: Sketch of myosin-II and its function. (a) Scheme of myosin-II: molecule is composed of two MHC having a coiled-coil structure, a non-helical tail and globular head group. The light chains are used to stabilize the neck for its use as a motor. (b) Function of a bipolar myosin-II filament bound to actin filaments that are able to create a pulling force relative to the actin filaments bt using the motor function of the myosin. Reprinted from (19), Copyright (2007), with permission from Elsevier.

in the order of appearance during cell migration and in Figure 2.5 we show a sketch of the process including the migration steps and modules needed, namely the actin cytoskeleton, myosin motors and adhesion areas.

We assume a cell sitting on a substrate (2.5 A). To start its migration, it begins to polymerize a branched actin network that is responsible for a local protrusion of the cell membrane (Figure 2.5 B). At this time the cell body that is adherent to the substrate and is barely moving. The protruding part of the cell - called the leading edge - contains a high concentration of freshly polymerized actin whereas the concentration of myosin-II motors is reduced.

After this actin-rich protrusion, also called the pseudopodium, has formed, it has to 
contact the substrate and form new adhesion sites (Figure 2.5 C). The actin cortex is now under a strong tension. This helps the cell to break the bonds of adhesion at the trailing edge (Figure 2.5D). Finally to shift the cell body, the cell needs to pull in the posterior, which is done by a contraction of myosin-II motors inside the actomyosin network. The sliding of the myosin-II motors against the actin filaments can, due to the connection of the actomyosin cytoskeleton to the adhesion sites, generate a traction force that allows the cell to migrate forwards (2). After this contraction, the cell has undergone a net displacement (Figure 2.5E) and is again in the same phase as in Figure 2.5 A. From here it can start polymerizing an actin network again. (Figure $2.5 \mathrm{~F}$ )

In amoeboid migration, the front at extension called the pseudopodium is essential for cell migration in $D . d$. In the following section, we will describe this crucial part of the migration machinery in more detail.

\subsubsection{Pseudopods}

Next to blebbing (80), pseudopods are the most prominent feature of amoeboid migration from a macroscopic point of view. Besides describing cell migration as a biochemical process, in which you identify every molecule and its interaction network, you can also start from geometrical optical observations. This way to describe amoeboid cell migration is also referred to the pseudopod-centered approach (82).

It is possible to distinguish between two types of pseudopods. Due to their origin, we name one kind splitting pseudopod, as it is formed close to an already existing pseudopod and usually alternating sides at an angle of about $55^{\circ}$. This zig-zagging 

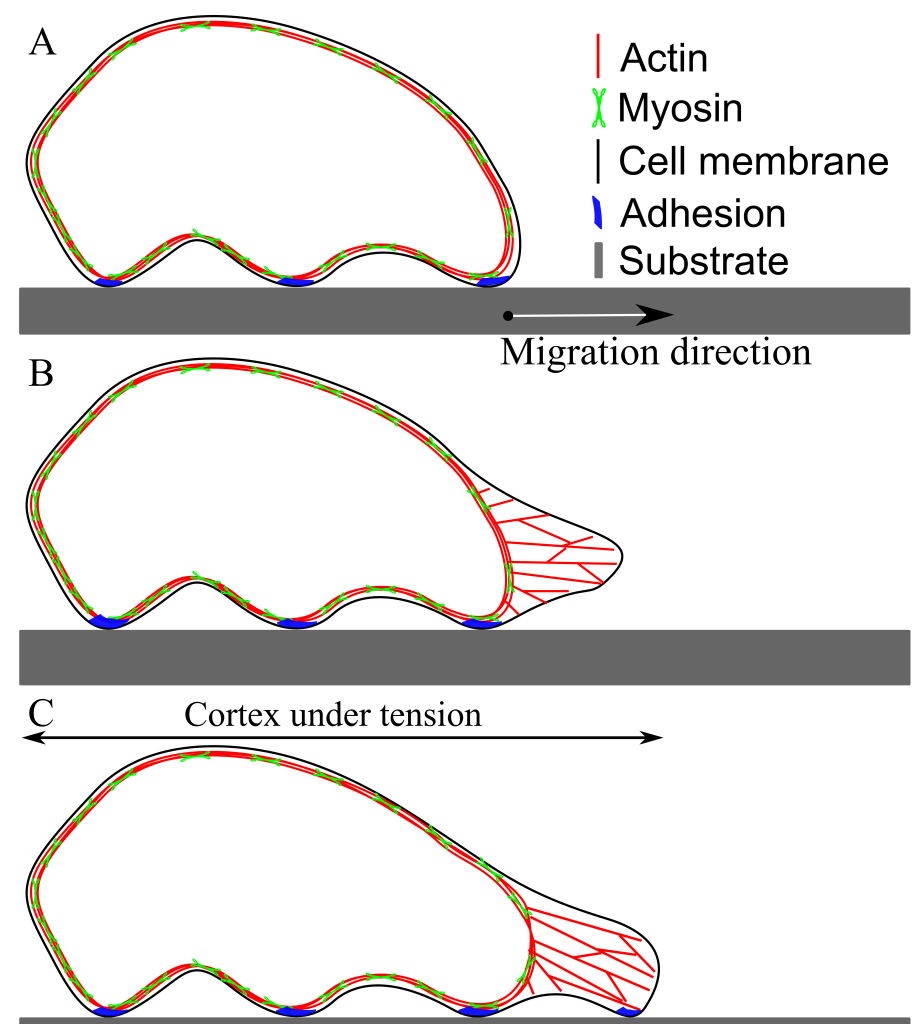

$\mathrm{D}$
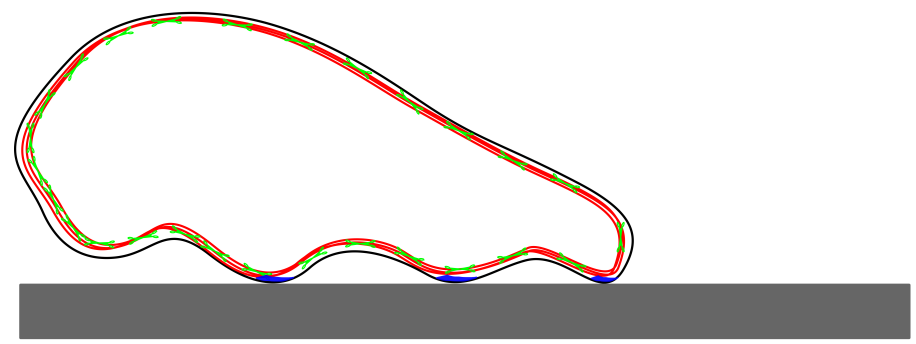

$\mathrm{E}$
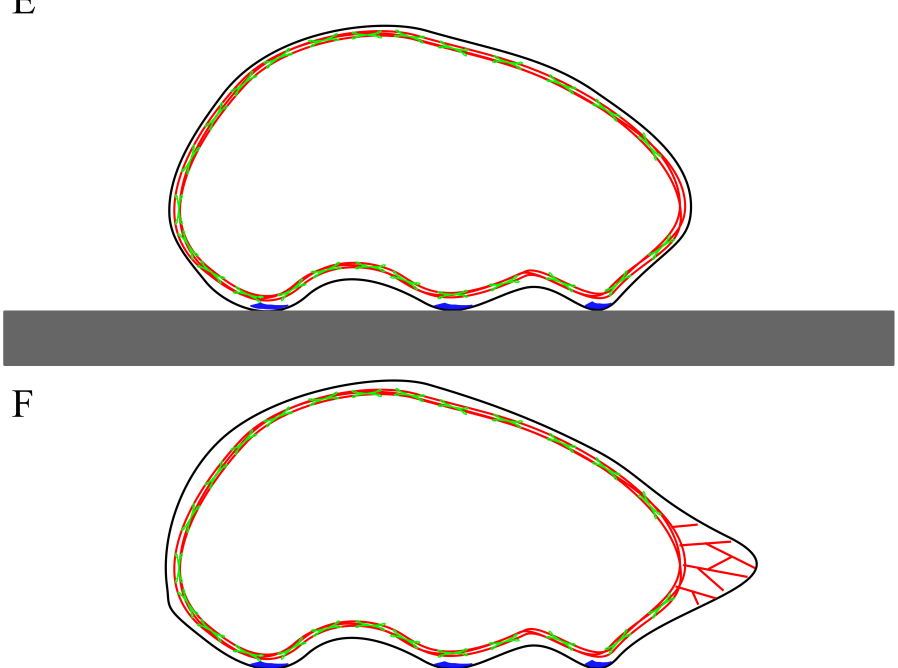

Figure 2.5: Migration mechanism of amoeboid cell. A Cell starts to migrate. B Pseudopod is formed due to branching of the actin network. The cell membrane at the leading edge is pushed forward. $\mathbf{C}$ The new pseudopod is adhering to the surface what creates a tension in the cell cortex. D Due to tension in the cell cortex adhesion bonds at the trailing edge break. E Myosin-II contraction pulls the trailing back inside. F Cell forms a new pseudopod. Sketch adapted from (1) and (2). 
manner of cell migration can be compared to ice-skating. The second type of pseudopod is the so-called de-novo pseudopod. In contrast to splitting pseudopods, the position of a newly formed de-novo pseudopod shows no angle-dependence (13). To clarify the differences between the two kinds of pseudopod formation, we have to discuss the migrational modes of the cells. It is well-known that the differences in chemical gradients and cell development cause differences in the cell migration. Regarding an unpolarized $D . d$. cell (vegetative or in early stage of development) that is able to migrate, we will find no clear front and back and it will extend pseudopodia in every direction (73). Apart from that, it will react to a chemoattractant gradient by forming a pseudopod in the direction of highest cAMP concentration. If the position of the chemoattractant will be changed, it will form a new pseudopod in the new direction.

If a $D . d$. cell is in its chemotactically active developed phase for a longer period, typically after 6 hours, it tends to be more polarized, hence it has a front to back asymmetry ( $(\underline{88})$. In a chemoattractant gradient the formation of pseudopods will still be at the leading edge, but they will orient in the gradient. Hence the cell will make a so-called u-turn, due to its already reached polarization. This polarization is produced by the symmetry breaking of signaling membrane lipids phosphatidylinositol (3,4,5)-trisphosphate (PIP3) and phosphatidylinositol 4,5-bisphosphate (PIP2) (84), which we will describe in more detail in section 2.3.1. 


\subsection{Chemotaxis}

The chemotactic migration of single amoeba during the aggregation of $D . d$. is a crucial part of its social cycle. Additionally, gradient sensing and amoeboid motion are of more general interest because of their importance for example in the human immune system as well as for the metastasis of cancer.

This very robust, evolutionary conserved mechanism of chemotaxis will be described in more detail in the following section and will be linked to the aforementioned migration modules.

\subsubsection{Chemotactic Signaling Network}

The chemotactic signaling cascade of $D . d$. is very complex and has been the subject of intensive research especially during the last two decades $(45,84)$. This research has unveiled four somewhat redundant signal modules merged into the chemotactic signaling system (73).

The main parts of the $D$. $d$. signaling cascade are shown as an overview in figure 2.6. To gain some understanding of the full complexity, more important modules for this thesis will be discussed in greater detail below.

The natural way to describe this complex and heretofore not fully understood pathway is to start at the receptor level. The chemoattractant cAMP can be sensed by $D . d$. cells with G-protein coupled receptors (GPCR), namely the four receptors of cAMP receptors cAR 1-4 (46). Details about the receptor affinities and the functionality of the four different receptors can be found in literature $(24,45)$.

As the name already explains, these receptors are coupled to heterotrimeric Gproteins that are responsible for transforming the exterior chemical signal to a 


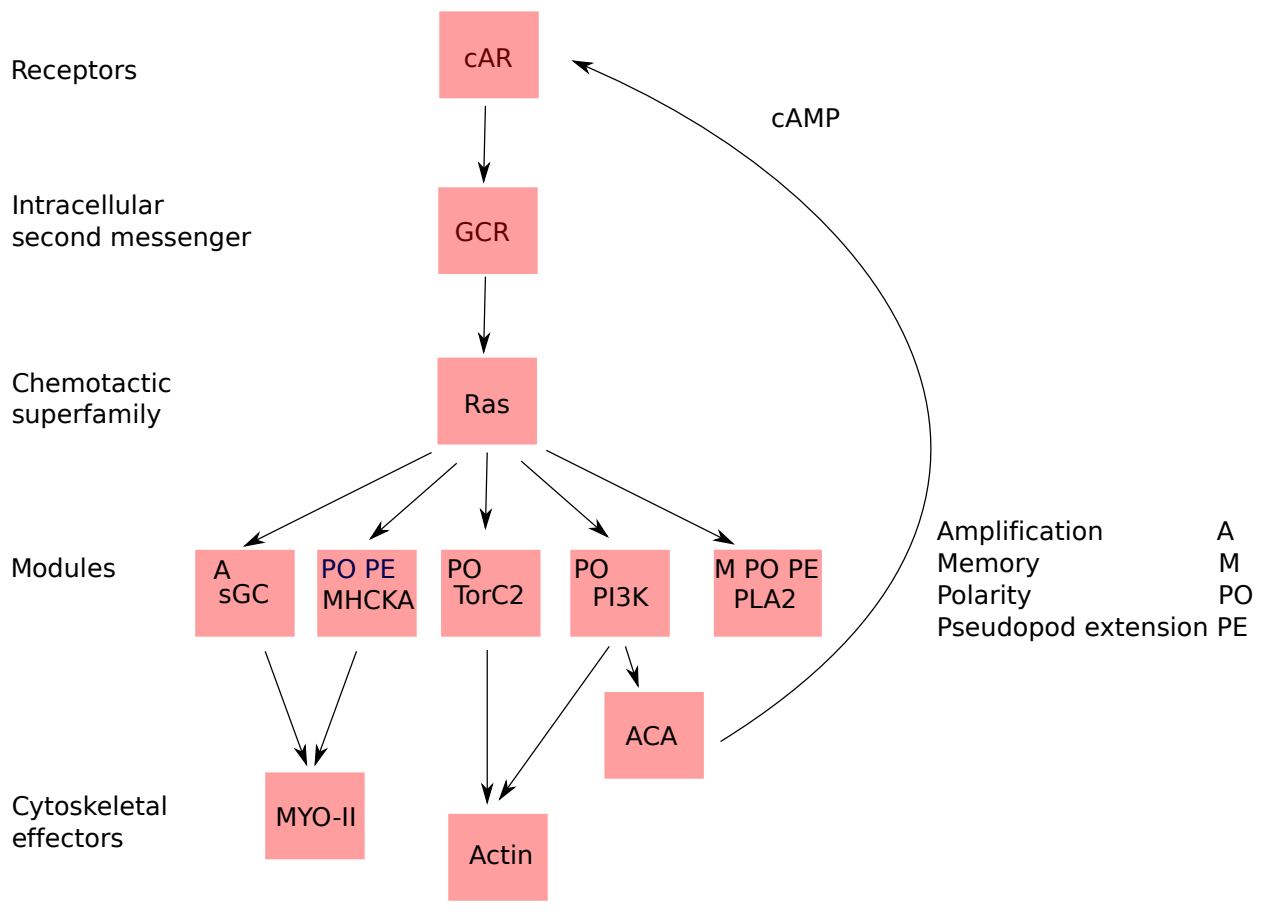

Figure 2.6: Overview of most important modules in $D$. $d$. chemotactic signaling cascade. cAMP binds to a cAR receptor that dissociates the G-protein G $\alpha 2$ GDP-G $\beta \gamma$ into its two parts, when occupied with a cAMP molecule. This Gproteins transfer the extracellular signal into an intracellular cascade activating several Ras proteins. These Ras proteins on their own activate several pathways that lead to symmetry breaking, actin polymerization, myosin-II contraction and cAMP production. Sketch redrawn according to (48).

cytoplasmic signal. To understand this process in more detail, we show a sketch of the signaling cascade from the cAR receptors to the $G \alpha 2-G T P$ in Figure 2.7. When a cAMP molecule binds to the cAR receptor, with highest affinity to cAR1, the G $\alpha 2$ GDP-G $\beta \gamma$ complex dissociates into G $\alpha 2$-GDP and $\mathrm{G} \beta \gamma$. Within this dissociation step, G $\alpha 2$-GDP is transferred to its active form G $\alpha 2-\mathrm{GTP}$. Both the G-proteins play important roles in the further downstream signal relay. The system controlling the occupancy of the cAR receptor involves the nonreceptor Guanine nucleotide Exchange Factor (GEF) and GTPase-Activating Proteins (GAP), the protein resistant 


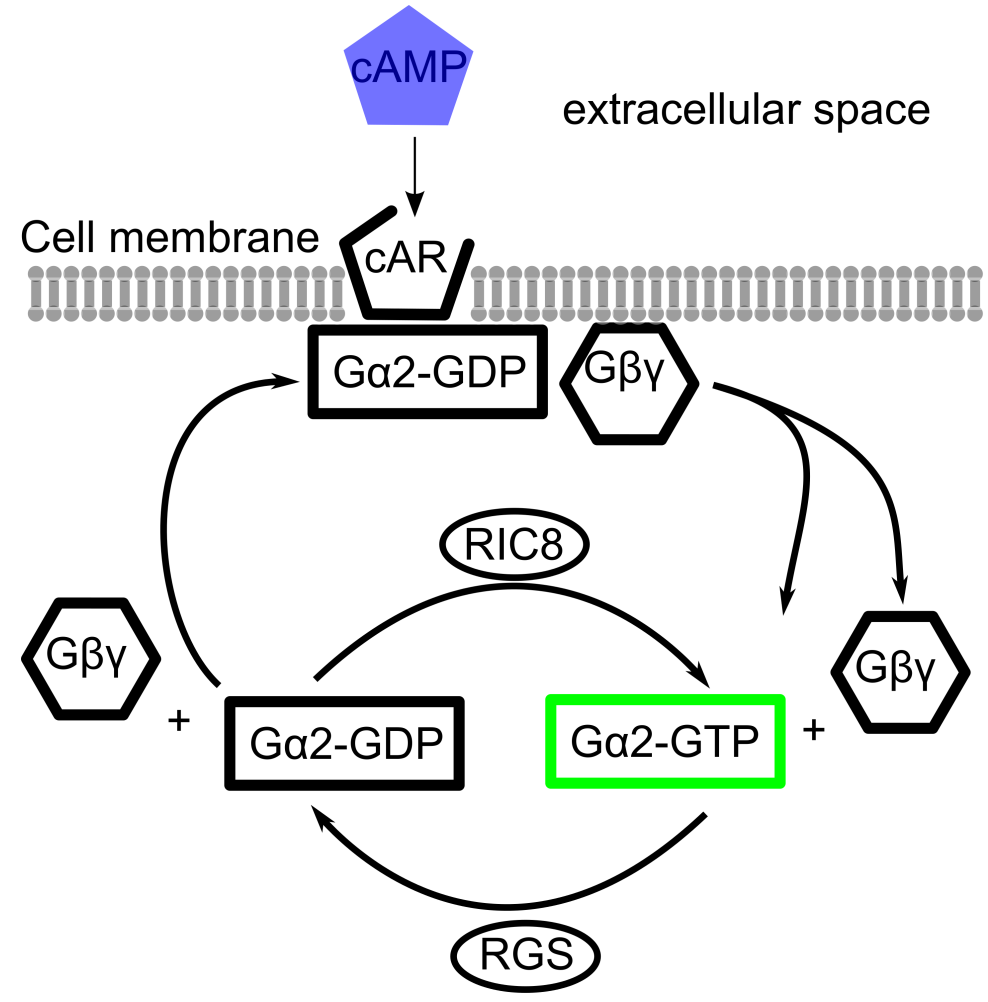

Figure 2.7: Sketch of transduction of cAMP-signal to Ras-GEF. The binding of the cAMP molecule to the cAR receptor induces dissociation of the coupled Gproteins G $\alpha 2$-GDP-G $\beta \gamma$. RIC8 and the RGS influence the amount of G $\alpha 2$-GTP that then is able to activate downstream Ras proteins. Active forms marked in green. Sketch redrawn according to (43).

to inhibitors of cholinesterase 8 (Ric8), and regulators of G-protein signaling (RGS proteins).

While Ric 8 is able to stimulate the conversion of $\mathrm{G} \alpha 2$ from its GDP into the GTP form, the RGS proteins assists the back reaction into the GDP form. More details on the general properties, like the protein structure of the G-proteins can be found in (53).

A class of second messenger proteins of main interest in the signaling cascade of $D$. $d$. is the Ras super family, which consists of 15 proteins, including 11 Ras, 3 Rap 
and one Rheb related protein (50). Both the $\mathrm{G} \alpha$ and $\mathrm{G} \beta \gamma$ are involved in activating different Ras proteins. Specific Ras proteins play a crucial role in basically every part of the chemotactic signaling pathway that is known so far $(50,73)$. We will focus on most important Ras proteins for chemotaxis, Ras-C and Ras-G are important in chemotaxis (10), Ras-G and unspecifically also Ras-C can be visualized with the fluorescent marker Raf1-RBD-GFP, that will be described in section 2.3.3.

The influence of Ras-G and Ras-C on $D . d$. chemotaxis is widely studied in the literature and demonstrates not only the importance but also the complexity and the various interactions of the different signaling pathways responsible for chemotaxis (10, 42, 47, 67), for instance it was shown that Ras-C-GTP is also necessary for the formation of cAMP (50). Ras-C-GTP activates cytosolic regulator of adenyl cyclase (CRAC) that itself regulates the adenyl cyclase (ACA), which produces cAMP (40). Research shows that both proteins have overlapping functions (50). The process of most interest for this thesis that involves Ras-G/C, concerns the symmetry breaking due to phosphatidylinositol (3,4,5)-trisphosphate (PIP3), as shown in Figure 2.9.

In this process of symmetry-breaking, there are four constituent of major importance. The membrane lipid PIP3 can be dephosphorylated by the phosphatase and tensin homolog (PTEN) into PIP2. The phosphorylation of PIP2 to PIP3 is assisted by the kinase phosphatidylinositol-4,5-bisphosphate 3-kinases (PI3K). In the chemotactic signaling cascade, the activated Ras-GTP forms regulate the PI3K activity (47).

PIP3 is able to activate cytoskeletal mediators like SCAR/WAVE. Thus the signaling cascade directly interacts with the actin cytoskeleton leading to its remodeling and finally to migration. 


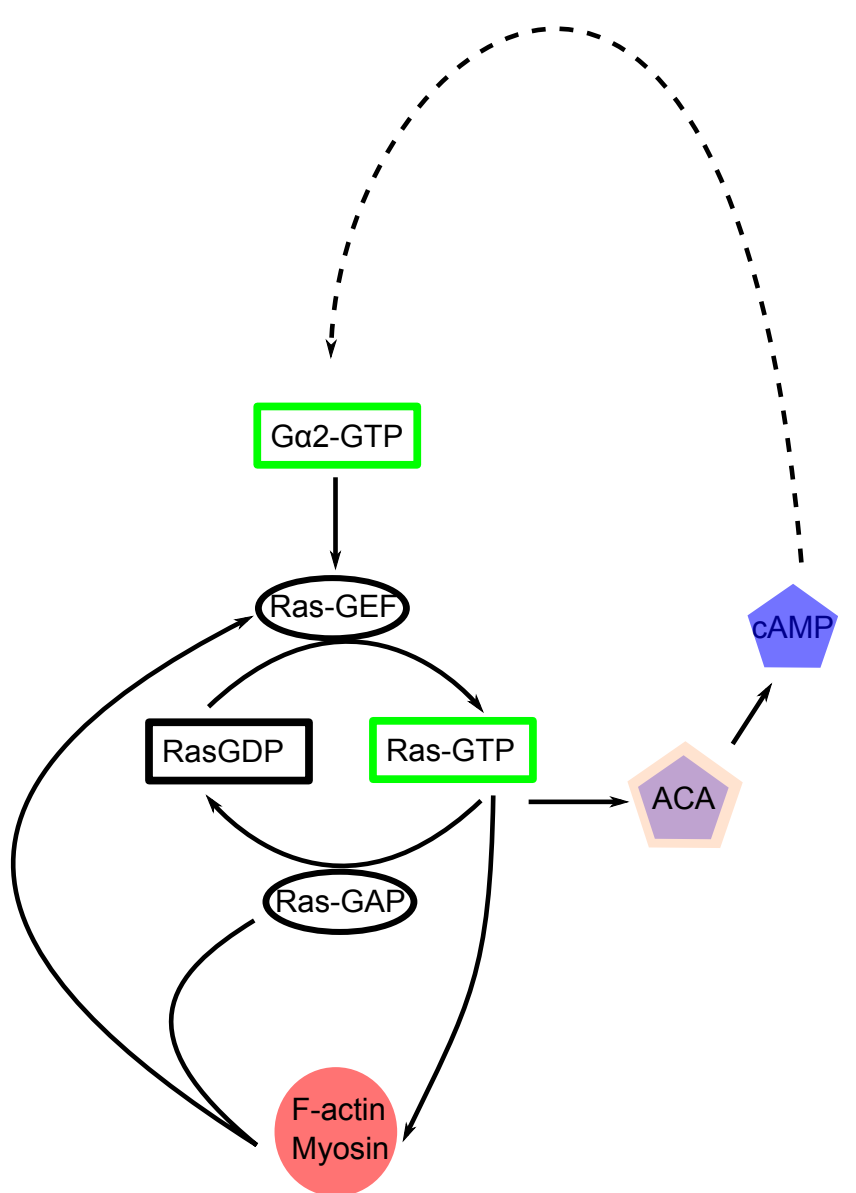

Figure 2.8: Sketch of transduction of signal from $G \alpha$ to actin polymerization via Ras. The upstream produced G $\alpha 2$-GTP activates Ras-GEFs that are able to catalyze the exchange of GDP for GTP in Ras proteins. The active Ras-GTP forms are able to activate downstream the production of cAMP due to ACA as well as actin polymerization and Myosin contraction. Active forms marked in green. Sketch redrawn according to (43).

\subsubsection{Chemotactic Migration}

The intent of this section is to combine the concepts introduced above into a consistent picture of chemotactic migration. A model of the cell that includes parts of the actomyosin machinery, parts of the signaling pathway and the cell geometry is expected to capture the complexity of this whole process and give an overview of the situation. 


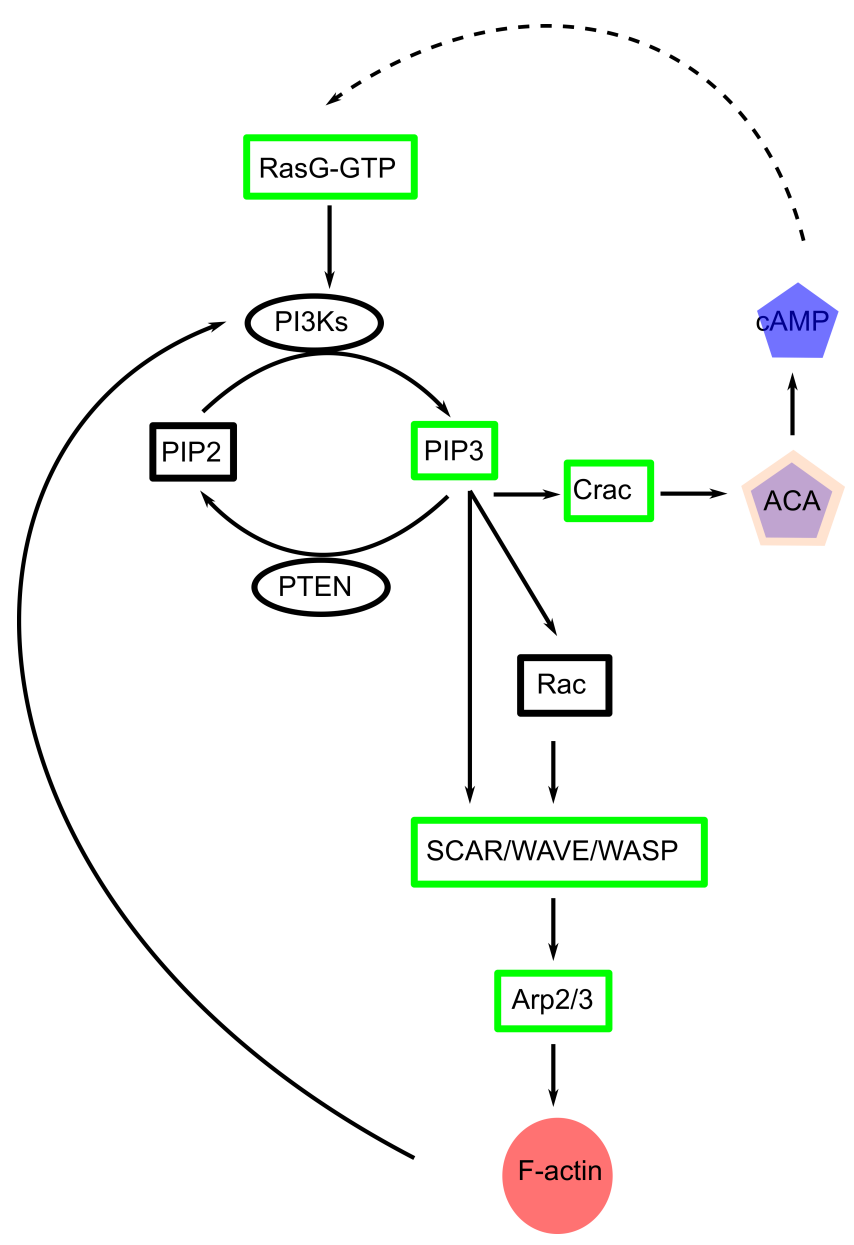

Figure 2.9: Sketch of signal transduction from RasG until actin polymerization via PIP3. RasG activates the PI3 Kinase leading to increase of PIP3. PIP3 itself can activate the cAMP production via CRAC and ACA and on the other hand it activates SCAR/WAVE as well directly as additionally via the Rac pathway. In such a way, the Arp $2 / 3$ complex is activated and a branched network of actin filaments will be the result. ACtive forms marked in green. Adapted from (47).

The cell is exposed to a gradient of the chemoattractant cAMP, as shown in Figure 2.10 by the blue pentagons that represent the cAMP molecules increasing in concentration from the right to the left. These molecules bind to membrane bound cAR-receptors and hence activate the cell's chemotactic signaling network. The extracellular signal is transferred into an intracellular signal by the G-protein-coupled 


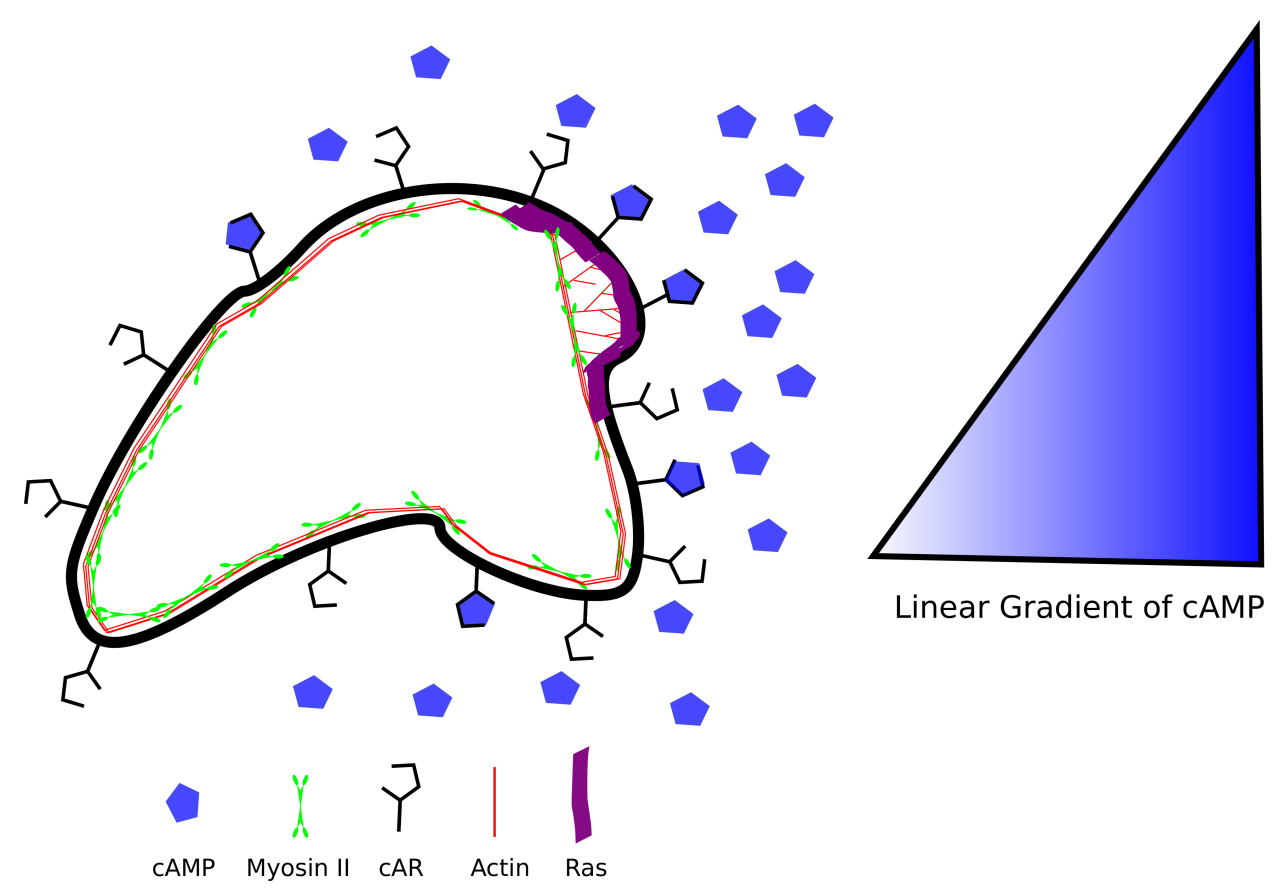

Figure 2.10: Sketch of chemotaxis in $D$. $d$.. As there are more cAMP molecules on the right-hand side more cAR receptors are occupied there and the cell is oriented in this direction. An active Ras patch is indicated at the pseudopod in the upper right side of the cell. The actin cytoskeleton is close to the membrane everywhere apart from the pseudopodium, where a branched actin network is formed to push put the cell membrane. In this so-called leading edge no myosin is incorporated in the actin cytoskeleton. Contrary to the rear of the cell, where it is responsible for the retraction of the back of the cell.

receptors cARs. The internal signal processing via the Ras protein superfamily (shown in purple) leads to a symmetry-breaking of the distribution of molecules involved in the chemotactic signaling cascade, especially the PIP2/PIP3 localization and the activation of the actomyosin cortex. Finally, the actomyosin machinery leads to membrane propulsion (pseudopodia) and cell migration. 


\subsubsection{Genetic Mutations}

A very important advantage of using $D . d$. is the knowledge of the fully sequenced haploid cell genome (29), leading to the capability of controlling specific genes. This control is the basis of understanding the dynamics of the signaling cascade as we are able to label specific proteins fluorescently genetically.

A way of interfering minimally with the intrinsic dynamics of $D . d$. cells while investigating them is to use indirect labels.

The two fluorescently labeled protein probes that are of utmost importance for this thesis are LimE and Raf1-RBD. The LimE protein colocalizes with freshly polymerized filamentous actin. It has two advantages: a direct labeling of all actin molecules would lead to a saturation and it would be challenging to obtain precise measurements of F-actin as the background of G-actin would lead to a poor signalto-noise-ratio. The more important effect considers the rate constants, such as the diffusion rate, as they may decrease if one would add a bulky fluorescence tag like a GFP protein on the actin monomers (15).

The other indirect fluorescent label that is used in this thesis is the Ras Binding Domain of human Raf1 tagged with GFP (Raf1-RBD-GFP) (91). This protein labels Ras-G and Ras-C as it colocalizes with Ras-G in its GTP-form. Therefore Ras- $\mathrm{G}$ is more specifically labeled and fluorescent signals correspond to more than $80 \%$ to Ras-G activity. Hence we can visualize the Ras proteins in their active form, during signaling. 


\subsection{Theoretical Descriptions}

The chemotactic signaling of $D . d$. has been a main research interest for many years as well with experimental techniques as with computational models. Step by step a wide knowledge of the biochemistry of chemotaxis in $D$. $d$. was gained. In this chapter we want to present the main results that are common knowledge in the literature along with the hypotheses that are widely used.

Since the first publication of a mathematical model, explaining pattern formation in morphogenesis by Turing in $1952(78)$, the approach to model biological systems with partial differential equations has widely been used (35, 90).

The theoretical descriptions of pseudopod formation is also heavily dependent on which aspect of the problem is regarded. As already mentioned in subsection 2.2.2.1. the models are either based on cell signaling dynamics or based on microscopy and pseudopod formation. Hence we will introduce two models that may explain the system of extending pseudopods in $D . d$. chemotaxis.

\subsubsection{Activated Membrane Patches}

A specific model commonly used to explain the pseudopod formation based on cell signaling was developed by Hecht and collaborators $(\underline{35})$. In this model where activated membrane patches are responsible for the formation of pseudopodia. The emergence of these patches is due to an reaction diffusion system. In the simulation, they link an external compass with an one-dimensional excitable medium on the cell membrane. The excited patches on this membrane regulate the actin polymerization and lead to the shape-changes and hence to the cell migration.

In order to describe the model in more details, we will show the equations in the 
following paragraph. It is an excitable reaction-diffusion model related to the cell membrane. The model is a spatially distributed Fitzhugh-Nagumo model that can lead to patches of the activator, dependent on the parameters.

$$
\begin{aligned}
& \frac{d a}{d t}=D_{a} \Delta a+\frac{1}{\epsilon}\left(a+b \cdot a^{2}-a^{3}-b\right)+\eta \\
& \frac{d b}{d t}=D_{b} \Delta b+a-\mu b+\beta
\end{aligned}
$$

The variables $a$ and $b$ are the activator and the inhibitor respectively, the concentrations of the two chemicals of the reaction-diffusion system, and $D_{a}, D_{b}$ are the diffusivities of $a$ and $b$. By $\epsilon, \beta$ and $\mu$ constants are denoted, while $\eta$ corresponds to a noise term.

The pseudopod formation is modeled by an activator dependent force, acting on the cell membrane. If the reaction-diffusion system leads to a patch of the activator, this force will induce a propulsion of the cell membrane:

$$
F_{t o t}=f_{p}(a)-\gamma\left(\kappa-\kappa_{0}\right)-C_{1}\left(A-A_{0}\right)-\lambda v
$$

In this force term, $\gamma$ corresponds to the membrane rigidity, $\kappa$ to the curvature, $A$ to the cellular area and $v$ to the membrane velocity. The function $f_{p}(a)$ models the protruding force and has the following functional dependence:

$$
f_{p}(a)=C\left(a-a_{0}\right) \cdot \theta(G)
$$

The function $f_{p}(a)$ is positive if the value of $a$ exceeds a mean value $a_{0}$ and is zero if there is no G-actin left at the membrane. Hence one can see the influence of G-actin in this function and this leads to a conservation law for actin that is shown in 
equations 2.5 and 2.6 .

$$
\begin{aligned}
\frac{\Delta F_{i}}{\Delta t} & =\Gamma v_{i} \Delta l_{i}-\delta_{f} F_{i} \\
\frac{\Delta G}{\Delta t} & =-\sum_{i} \Gamma v_{i} \Delta l_{i}+\sum_{i} \delta_{f} F_{i}
\end{aligned}
$$

$F_{i}$ describes the F-actin at each node $i, v_{i}$ the membrane speed, $\Delta l_{i}=\frac{r_{i-1, i+1}}{2}$ the membrane length, while $\Gamma, \delta_{f}$ are constants.

Finally the time evolution is thus given by:

$$
\frac{d v}{d t}=F_{t o t}
$$

To also include chemotaxis into this model Hecht et al. implemented an internal compass, where $\phi_{\text {int }}$ is the compass direction of the cell:

$$
\phi_{\text {int }}=\phi_{\text {ext }}+\eta_{\phi}
$$

Here, $\phi_{\text {ext }}$ models the external direction of the chemoattractant gradient and $\eta_{\phi}$ is a directional noise. Due to this definition $\phi_{\text {int }}$ is the leading edge of the cell. At this leading edge the choice of $\beta<0.6$ is made and hence the system at the leading edge is excitable, while the back of the cell is not.

\subsubsection{Statistical Geometrical Theories}

A statistical way to model the pseudopod formation was introduced by van Haastert in one of his publications ( $(83)$. In that work, statistical properties of the microscopic 
phenomena were used to describe the process and answer the question if statistics would be sufficient to explain the system.

This theoretical description of the pseudopod extension of $D . d$. basically regards the pseudopods as self-organized entities, being formed regularly. The position of a new pseudopod is calculated depending on its kind, being a splitting pseudopod or a de-novo pseudopod. Additionally, the existence and the strength of a chemoattractant gradient is also of great interest for the model. In case of a splitting pseudopod, the direction of the new pseudopod is chosen to be identical to the direction of the previous pseudopod. This direction will be modified by a bias imposed by the chemoattractant gradient. To include noise, the direction is the mean value of a von Mises distribution with variance $\sigma_{\phi}$. The new direction is chosen randomly within the range of the von Mises distribution.

In case of a de-novo pseusopod, the initial direction will be picked randomly between $\left[0^{\circ}, 360^{\circ}\right]$. The following steps are exactly the same as for splitting pseudopods.

With this model, using the statistical properties of the pseudopodia, it is possible to describe the system and as well allowing to make predictions about knock-out mutants $(12,13)$. 


\section{CHAPTER 3}

\section{Experimental Setup and Methods}

In this chapter we will focus on the experimental setups and the methods used to preform our experiments. We will start by presenting the protocols used prepare the cells for the experiments. The second part of this chapter will include details on the experimental setups, namely the microfluidic devices and the involved microscopy techniques.

\subsection{Cell Culture and Genetics}

$D . d$. exists as several cell strains that need to be treated differently. An important difference between strains is the ability to use different food supplies. The cell lines used in this thesis are all axenic, meaning that they are able to feed on medium (HL5 (Formedium, Norwich, England)) free of other organisms (87). A more recent protocol of $D . d$. cell culture can be found in $(\underline{31})$. Cells are stored either as spores or directly as cells at $-80^{\circ} \mathrm{C}$ for extended time periods. To use the cells for experiments, 
frozen stock is thawed at room temperature and afterwards they are cultured in HL5 medium on Petri dishes. The doubling time of the cells is between 8 to 9 hours at the optimal growing temperature of $21-23^{\circ} \mathrm{C}$. The cell culture has to be subcultured every 2-3 days, when the cells have become confluent on in the Petri dish. The passage number is increased by one each time for a new subculture and the cells will be discarded after passage 15 , due to the increasing probability for a genetic change of the culture.

To perform experiments, we use cells harvested in their exponential growth phase, which are due to the pulsing with cAMP in their highly chemotactic phase. The preparation of the cells starts one day before the experiment. The preparation process starts with pipetting $10^{6}$ cells into a flask with $25 \mathrm{ml}$ HL5 medium. This flask is cultivated on a shaking table at $22^{\circ} \mathrm{C}$ with 150 rotation per minute. On the day of the experiment, 7 hours prior to the start of the experiment, the cells are centrifuged and the medium is removed. The cells are washed with phosphate buffer and afterwards centrifuged again. The remaining pellet is diluted with $20 \mathrm{ml}$ phosphate buffer and is positioned on the shaking table at $22^{\circ} \mathrm{C}$, when every 6 minutes a pulse of cAMP (approx. $60 \mu \mathrm{l}$ with concentration $18 \mu \mathrm{Mol}$; Sigma-Aldrich) is dropped into the flask to increase the chemotactical activity of the cells as reported in (18).

We also used mutant $D$. $d$. cells. For the curvotaxis project, ACA-Null mutant cells were used, because cells lacking the aggregation stage adenylyl cyclase (ACA) the cells are not able to produce cAMP and hence they are missing the capability to aggregate. Those cells possess the ability to perform chemotaxis but only towards external cAMP as they cannot porduce it. This missing functionality enables us to investigate the effect of the complex geometry without the strong influence of chemotaxis. 
To investigate the signaling cascade and its influence on the cell geometry, we use myosin-II-null and PTEN-null knockout cells. The knockout of myosin-II strongly interferes with the cell cortex, which is visible for instance through the fact that myosin-II-null cells are not able to divide without being attached to a surface (21). Hence after the pulsing, there are many multinucleated cells. Those will divide as soon as they are adherent on a substrate. The PTEN-null knockout is as described in section 2.3.1 interfering with the symmetry breaking of the chemotactic cells mainly. As one of the enzymes for the process is knocked out, the fine-tuned process is disrupted. It leads to cells that have less control of the size and positioning of pseudopodia and show decreased chemotactic efficiency.

To investigate the role of the protein Ras during chemotaxis, we need to image its dynamics. We accomplish this goal via genetic labeling. A common way is to use a marker protein that only interacts with the GTP form. Also important is the fact that the fluorescent label is not tagged directly to the protein itself because otherwise it may change its dynamics.

We used different labels designed by the group of van Haastert (85) and Gerisch/MüllerTaubenberger (32). To maintain cell lines with comparable properties e.g. the amount of fluorophores, we had to electroporate fresh wildtype cells every three to four weeks with the expression plasmid. After several days of recovery these genetically modified cells could be used similarly as standard wildtype cells. 


\subsection{Methods}

\subsubsection{Microfluidics}

An important methodological objective of this thesis is to stimulate cells with welldefined chemical stimuli using a microfluidic gradient mixer described in (41) and improved in (71). This precise tool allows us to control the gradient that the cell is able to sense. The devices is built in a stepwise procedure explained in the following: A chrome mask is used to prepare a silicon wafer via soft lithography to get a negative form of the microfluidic channel; in a subsequent step Polydimethylsiloxane (PDMS (Sylgard 184, Dow Corning Europe SA, Houdeng-Goegnies, Belgium)) is cast to produce the microfluidic channel walls. The final step is the bonding of PDMS form to a glass cover slip to seal the microfluidic channel.

\subsubsection{Soft Lithography}

The basic idea of soft lithography is to create small scale devices with very high precision mold. Our protocol to create the microfluidic channels is very similar to the methods described in the reviews (41) and $(\underline{89})$ and the individual steps can be found in Figure 3.1. In our case we create microfluidic flow chambers to apply precise environmental conditions to our biological sample. We used a chrome/quartz mask that was build from a CAD file several years ago by Song (CNF - Cornell Nanoscale Science \& Technology Facility).

Based on this chrome/quartz mask, we create a reusable master silicon wafer. After baking off residual oil or organics from a polished silicon wafer, we deposit the SU-8 photoresist on the wafer with a spin coater allowing the precise control of 
Idea $\rightarrow$ Chrome mask $\rightarrow$ Silicon wafer $\rightarrow$ PDMS molding $\rightarrow$ microfluidic channel

Figure 3.1: Basic idea of soft lithography. After the idea and the concept, using a CAD program a chrome mask will be created. This chrome mask is used to produce a silicon wafer which is the negative image of the PDMS stamp. The PDMS mold is used to built the microfluidic channel.

the height of the photoresist precisely ( $2000 \mathrm{rpm}$ for $35 \mathrm{~s}$ ).

After spin coating, the wafer is placed on a hotplate for $10-15$ minutes at $95^{\circ} \mathrm{C}$ for the soft baking process. The subsequent step is the exposure of the wafer to UV radiation below $350 \mathrm{~nm}$ wavelength with an exposure energy dose of $150-250 \frac{\mathrm{mJ}}{\mathrm{cm}^{2}}$ to crosslink the polymer photoresist. In this process, only the areas that are exposed to the light will be cross-linked and thus become the negative form of the structure.

The next step of wafer production is the post-exposure-baking (PEB) that is composed of two steps. To reduce the stress of the cross-linked film, the wafer is placed on a hotplate at $65{ }^{\circ} \mathrm{C}$ for one minute. Second, the wafer is placed for 3-5 minutes on the hotplate at $95^{\circ} \mathrm{C}$.

In the final step we reveal the photoresist structure by placing the wafer in SU-8 developer for about 8 minutes. This helps to dissolve the non-crosslinked SU-8, leaving behind the pattern. After this development, the wafer will be rinsed with fresh developer, iso-propanol and dried with nitrogen.

The procedure to create the microfluidic channels with a newly built wafer is presented in the following: The wafer is cleaned by rinsing consecutively with acetone, isopropyl alcohol and distilled water. Afterwards, Sylgard 184 PDMS Elastomer is mixed with Sylgard 184 Curing Agent (Dow Corning Europe SA, Houdeng-Goegnies, Belgium) at the ratio of 10:1 and poured onto the wafer that is situated in a weighboat. The PDMS in the weighboat will be degassed in an 
desiccator until all air bubbles have vanished. Finally the PDMS is cured for 60-90 minutes at $75^{\circ} \mathrm{C}$.

The cured PDMS, which can easily be cut afterwards with a sharp knife, is freshly cleaved and - at the designated spots - holes are punched into the PDMS to create inlets and the outlet of the microfluidic channel.

To seal the microfluidic channel a glass cover slide is attached after plasma cleaning both the PDMS and the cover slide for 1-3 minutes. To maximize binding strength the device is afterwards stored for 5 minutes at $75^{\circ} \mathrm{C}$.

\subsubsection{Microscopy}

The experimental work in this thesis relies on the use of microscopy and as the examined effects are on small length scales, it is required to use high resolution microscopy techniques like Confocal Laser Scanning Microscopy (CLSM). CLSM is a standard microscopy techniques with a high resolution. As described in Abbe's Law, the minimal resolution $d$ of a microscope is

$$
d=\frac{\lambda}{2 N A}
$$

where $N A$ is the numerical aperture $A=n \cdot \sin \alpha$. The wavelength $\lambda$, the refrective index $n$ and the half opening angle of the cone of light $\alpha$ are the important quantities in this equation and all have are bound in the small interval. It is possible to overcome this limit with special techniques, as described in $(9,36,37,64)$. But even to approach this limit with traditional techniques is far from trivial. As for widefield epi-fluorescence microscopy, the whole specimen is illuminated, and thus 
fluorophores of different layers, the images will contain light from all layers, which will decrease the resolution. The CLSM used in this thesis is a method that overcomes this problem and is presented in the following chapter. An enhanced CLSM, namely the spinnning disc Confocal Laser Scanning Microscopy (sdCLSM) will be explained, because, due to its high temporal resolution, we performed experiments with this setup.

\subsubsection{Confocal Laser Scanning Microscopy}

The main idea of Confocal Laser Scanning Microscopy is the introduction of a pinhole in the light path of the excitation laser. In this way, the excited volume is reduced to, in principal, the minimal spot to which light is able to be focused. Its size is around $200 \mathrm{~nm}$ in $\mathrm{x}$ - and $\mathrm{y}$-direction and $500 \mathrm{~nm}$ in $\mathrm{z}$-direction. The image quality is enhanced as no fluorescence is excited and collected from other spots.

The set-up of this microscope type is shown in Figure 3.2 that can be found at the homepage www.microscopyu.com.

The light path starts with the laser that is restricted to a very small focus by the light source pinhole aperture. Through a dichromatic mirror, the laser light is reflected to the objective that focuses the light to a spot on the specimen. There it will excite fluorophores, that will emit photons of a different wavelength. These photons will be focused by the objective on the photomultiplier in such a way that out-of-focus fluorescence will be blocked by the detector pinhole aperture. Hence one gets a fluorescent signal of a single point of the specimen. By scanning the probe you are able to image everything or just a part, for instance, single confocal planes of it. 


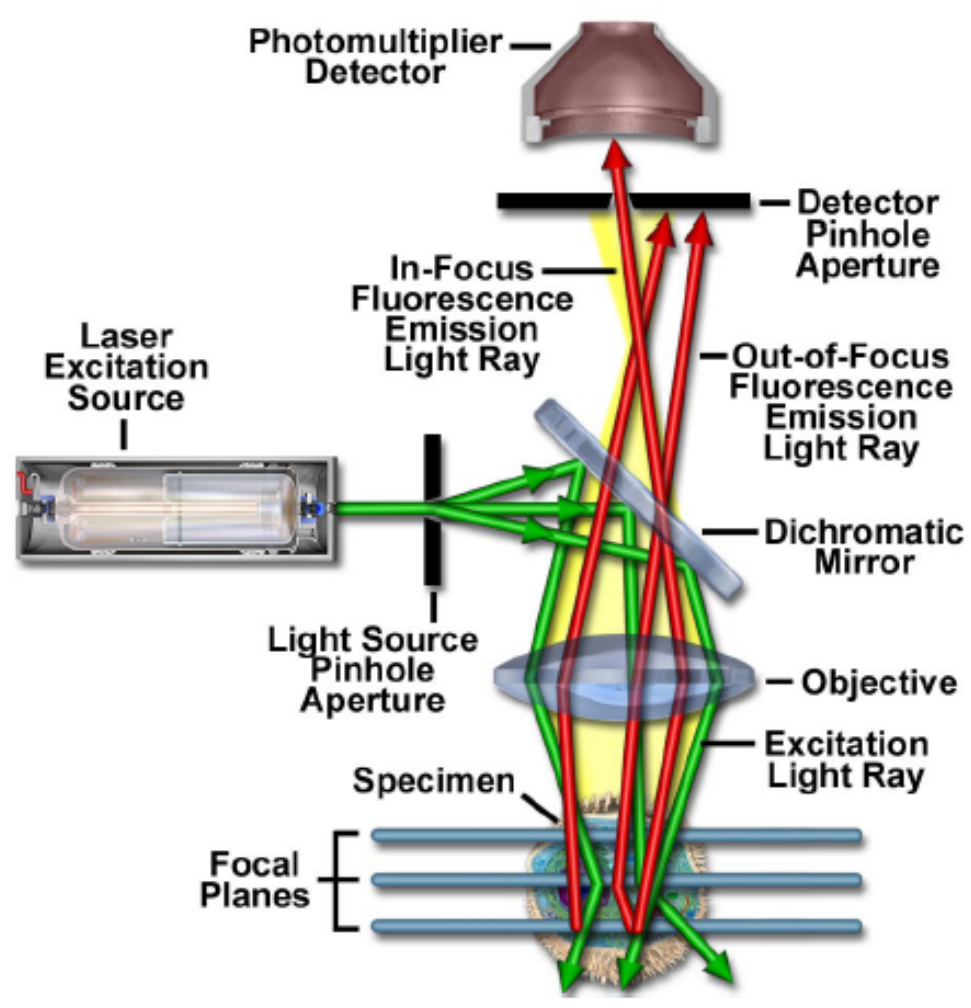

Figure 3.2: This sketch shows the lightpath of a laser scanning confocal microscope. The laser excitation source is shown on the left hand side. After passing a small pinhole the light is reflected by a dichromatic mirror through the objective onto the specimen. The fluorescent light, which is emitted by the specimen will be detected by a photomultiplier detector after having passed through the objective and the dichromatic mirror only if it matches with the detector pinhole aperture. That is if and only if it was emitted in the confocal plane that is visualized.

Taken from Nathan S. Claxton, Thomas J. Fellers, and Michael W. Davidson: http://www.microscopyu.com/articles/confocal/confocalintrobasics.html on July 21st, 2015. 


\subsubsection{Spinning Disc Confocal Laser Scanning Microscopy}

The sdCLSM is one special form of the confocal microscopy aimed at optimizing temporal resolution. The main difference between this technique and conventional CLSM is that a highly efficient camera is used to create the micrograph instead of the photomultiplier detector. To retain the confocality due to the precisely placed pinholes, the sdCLSM possesses two rapidly rotating discs, one incorporated with microlenses and the other one with pinholes $(72,76)$. In Figure 3.3 a sketch of the sdCLSM is shown which is taken from the web page of Zeiss-Campuss.de. The illumination of the specimen is done by focussing the laser with the microlenses of the first rotating disc to the pinholes of the second rotating disc and afterwards through the objective. Thus the confocality is obtained similarly as for the conventional CLSM. The emitted fluorescence will be focused through the objective and the pinholes, but in contrast to the excitation light reflected by the dichromatic mirror onto the CCD camera.

\subsubsection{Differential Interference Contrast Microcopy}

Within the variety of different microscopy techniques, differntial interference contrast microscopy (DIC), is a brightfield microscopy technique. The advantage of the DIC microscopy is that objects with volume-like cells can be imaged in a way that the height of the object can be visualized with a grey scale. On a DIC micrograph, one gets a pseudo three-dimensional image.

To accomplish this impression of three-dimensional imaging, the technique uses the interference of light due to a path differences while passing the probe, see Figure 3.4. Therefore the light has to go through a polarizer in the beginning. The polarized 


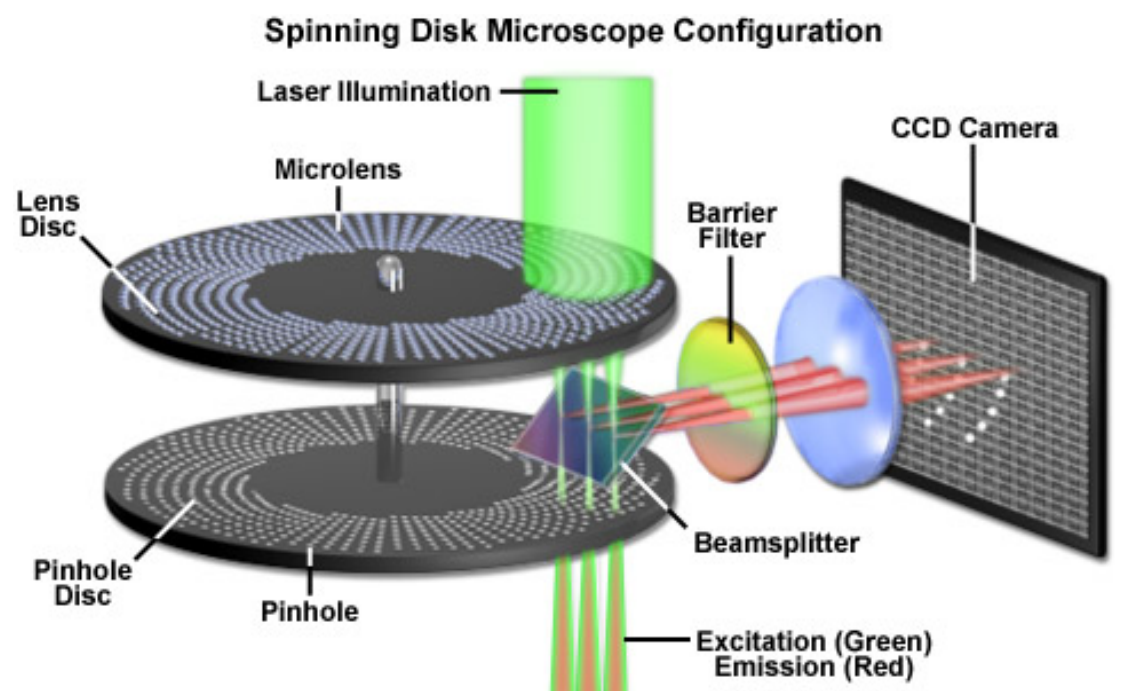

Figure 7

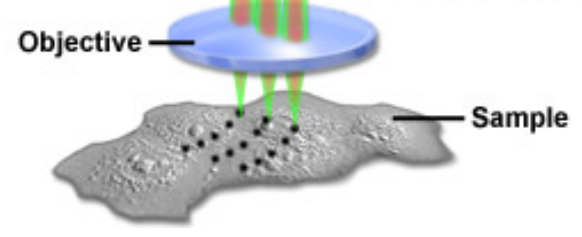

Figure 3.3: Sketch of Spinning Disc operation mode. The laser light is focused by the microlenses of the rotating lens disc through the pinholes of the rotating pinhole disc. Via the objective the specimen is illuminated. The emitted fluorescence of the sample takes the light path through the objective and pinhole disc before being reflected by a beamsplitter onto a CCD camera.

Taken from http://zeiss-campus.magnet.fsu.edu/articles/spinningdisk/introduction.html on July 21st, 2015.

light will be split by a Wollaston (Nomarsky) prism into two light paths. Both light paths will illuminate the specimen after a condenser. Due to the objective the two light paths will be merged into a second Wollaston (Nomarsky) prism and there they will interfere with each other. Dependent on the difference of the optical path which the light had to take, it will be constructive or destructive interference. Hence the image will be brighter or darker. As last step before the eze piece or the camera detector there is a analyzer that assures only correctly polarized will be detected. 


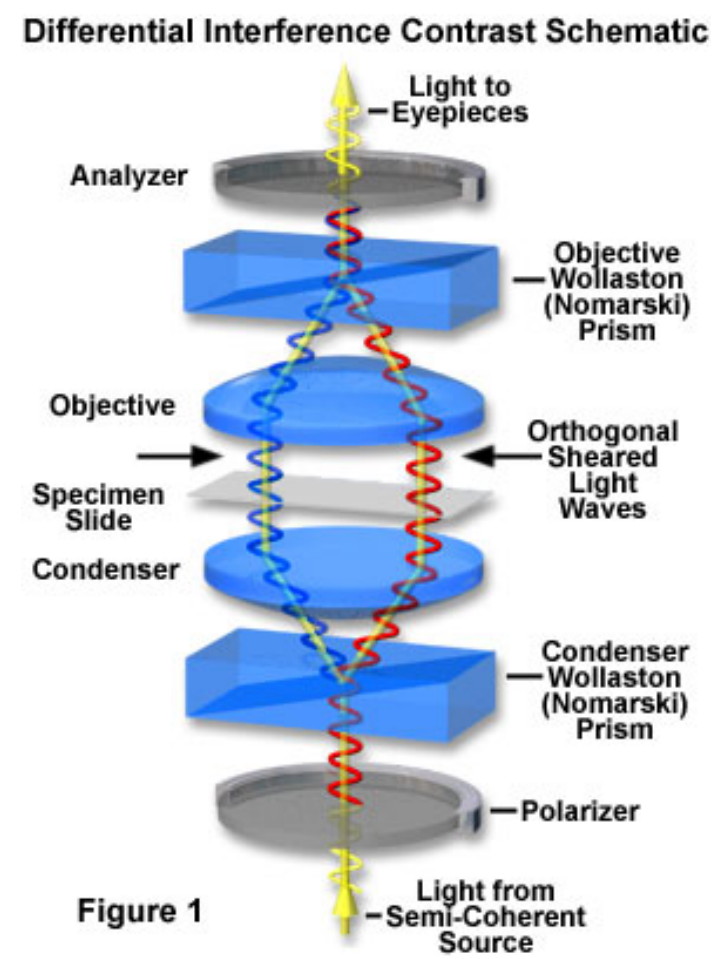

Figure 3.4: Sketch of DIC light path. Light is polarized and then splitted by a Wollaston(Nomarsky) prism. After the actual imaging of the probe with a condensor and an objective with a second Wollaston(Nomarsky) prism the two light paths are merged again and can interfere. Due to the analyzer only the correctly polarized light can pass to the ezepieces or the camera.

Taken from http://www.olympusmicro.com/primer/techniques/dic/dicoverview.html on July 21 st, 2015

\subsubsection{Experimental Setups}

For the different aspects of the experimental questions we want to address in both parts of this thesis, we have highly specialized experimental setups. To probe the signaling of chemotactically migrating $D . d$. cells, we use a diffusive mixer developed in Whitesides group several years ago (41) and was improved for the use with $D . d$. in our group (71).

Furthermore we established an experimental approach to study cell migration on curved substrates. Therefore we use an approach based either on optical fibers or - 
in cooperation with Marco Rivetti and Oliver Bäumchen - wrinkled substrates made of soft polystyrene films supported by PDMS layers. In the following these setups will be introduced in more detail.

\subsubsection{Curvotaxis Setup}

To find a more realistic description of geometrical effects underlying amoeboid cell motion and the associated pseudopod dynamics, it is necessary to investigate the cellular behavior in more complex environments than flat glass surfaces. A straightforward way to increase the complexity of the system is to consider three dimensions, albeit keeping the structure simple. Until now, experiments from migration literature were mostly restricted to flat surfaces. Unfortunately, this setting does not resemble the real environment of cells. When $D$. $d$. aggregate or prey on bacteria in the soil, they face a porous, often fluid filled, anisotropic and heterogeneous environment and hence have to crawl over curved surfaces, a situation distantly related to the one a neutrophil experiences in blood vessels at their curved interfaces. Therefore, in cooperation with Humboldt-Bessel-Fellow Oliver Steinbock (UF Tallahassee), we started to analyze $D . d$. cells migration on glass capillaries as done before for fibroblasts (63). To exclude chemotactical migratory effects we place the optical fibers in a perfusion chamber (RC-27, Large Bath Chamber, Warner Instruments, Hamden, CT, USA) on a glass spacers to allow a fluid flow around the fiber which washes away the cAMP molecules secreted by the cells. A sketch of this setup can be seen in Figure 3.5. We can image the fiber surface facing $D . d$. in this device with an inverted optical setup as well as with a top-view configuration. We use a peristaltic pump (RP-1 Peristaltic Pump, Mettler Toledo Inc., Columbus, Ohio USA) to create 
a fluid flow with a mean flow speed in the chamber of $v_{\text {flow }}=167 \frac{\mu \mathrm{m}}{\mathrm{s}}$. To investigate the actual velocities of the fluid flow close to the fiber, we used fluorescently labeled polymer beads Duke 36-6 (Polystyrene Divinylbenzene (PS-DVB), Duke Scientific Corporation, California, USA), with a diameter of $33 \mu \mathrm{m}$. The mean bead velocity close to the fiber was $v=10 \frac{\mu \mathrm{m}}{\mathrm{s}}$. Hence we can be sure that the velocity in the setup is not inducing migration of the $D . d$. cells but is still high enough to flush away cAMP, compare the velocity in other commonly used microfluidic devices as the gradient mixer $\left(v=650 \frac{\mu \mathrm{m}}{\mathrm{s}}\right)(71)$ or the linear microfluidic channel used for flow photolysis $\left(v=67 \frac{\mu \mathrm{m}}{\mathrm{s}}-110 \frac{\mu \mathrm{m}}{\mathrm{s}}\right)(\underline{8})$.

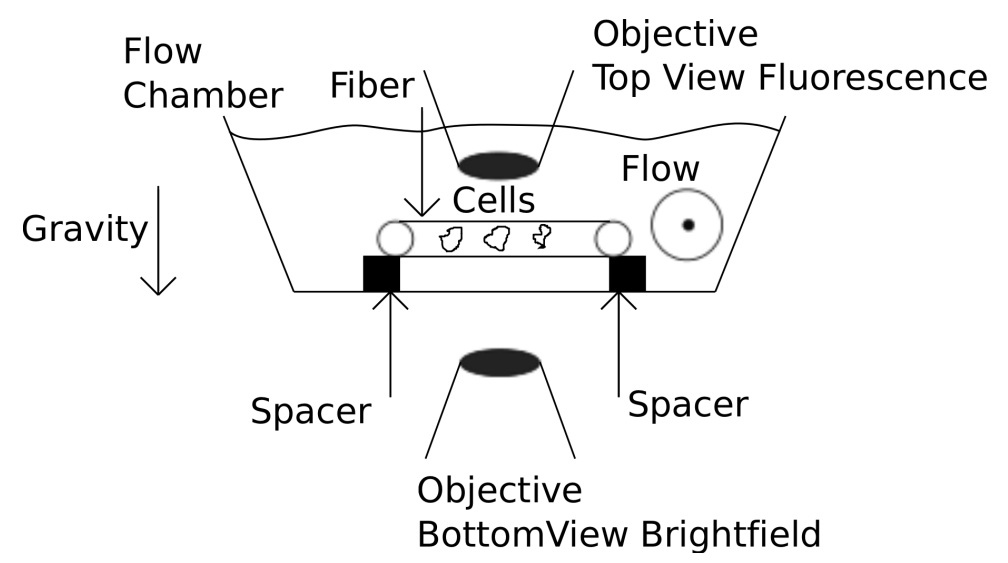

Figure 3.5: Sketch of the curvotaxis setup. The optical fiber is placed on two glass spacers inside the perfusion chamber. The perfusion chmaber is connected to a perfusion pump. Cells are added to the fiber from the top. Imaging can be done as well with inverted as with top-view optical setups.

The drawbacks of this setup are the lensing effect of the fiber and also the small area we are limited to, including problems arising with confocality of the used CLSM setup and the fluorescence readout. In cooperation with Oliver Bäumchen and Marco Rivetti, from the Department of Complex Fluids of Prof. Herminghaus, we designed wrinkled patterns to have a large area device with controlled amplitudes 


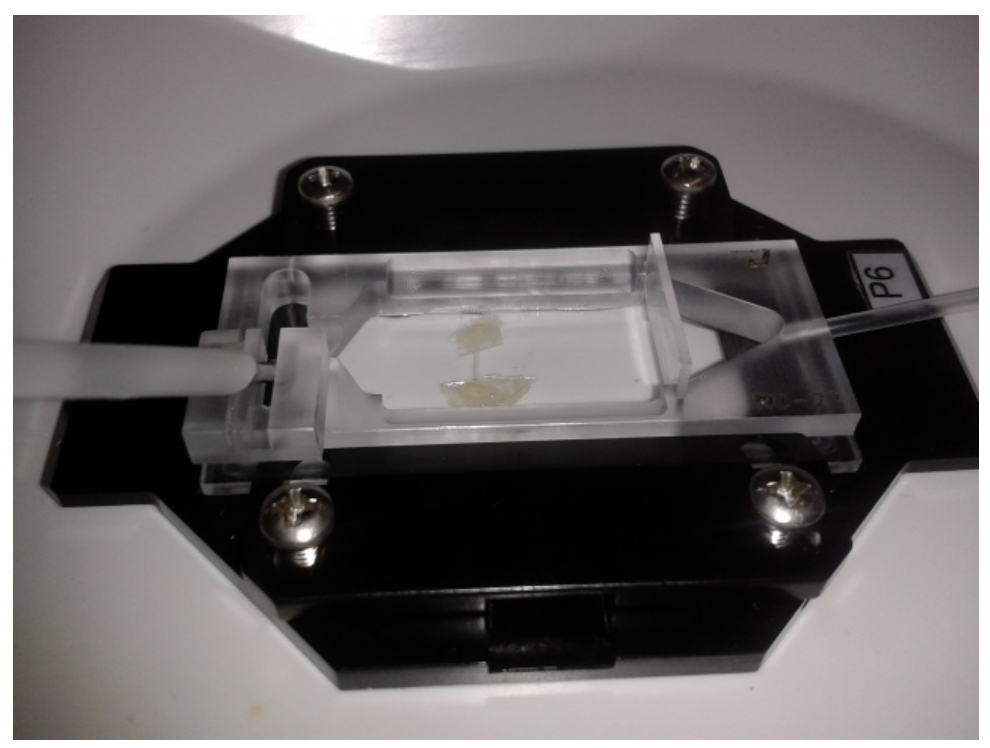

Figure 3.6: Photograph of Perfusion chamber, fiber fixed on spacers perpendicular to flow direction. The dimensions of the chamber are $5.1 \mathrm{~cm}$ in length and $2.6 \mathrm{~cm}$ in width. The flow is applied with a peristaltic pump to pump fluid in on the right side and suck fluid out on the left side.

and wavelengths, hence also curvatures. For this purpose we use a technique first described by Cerda and Mahadevon (16). We bind a thin, stiff polystyrene layer to a thick, soft PDMS gel that is under tension. After the two layers are bond to each other, the bending stress is released causing a wrinkling instability in the system. As calculated by Cerda and Mahadevan (16), the wavelength of the wrinkles is proportional to the bending stiffness over an effective elastic foundation of stiffness to the power one fourth. The amplitude of the wrinkles $A$ is proportional to the wavelength $\lambda$ and the imposed compressive strain $\frac{\Delta}{W}$, which is the fraction of the imposed compressive transverse displacement $\Delta$ divided by the width of the sheet $W$. The wavelength itself is dependent on the bending stiffness $B$ and the effective elastic 


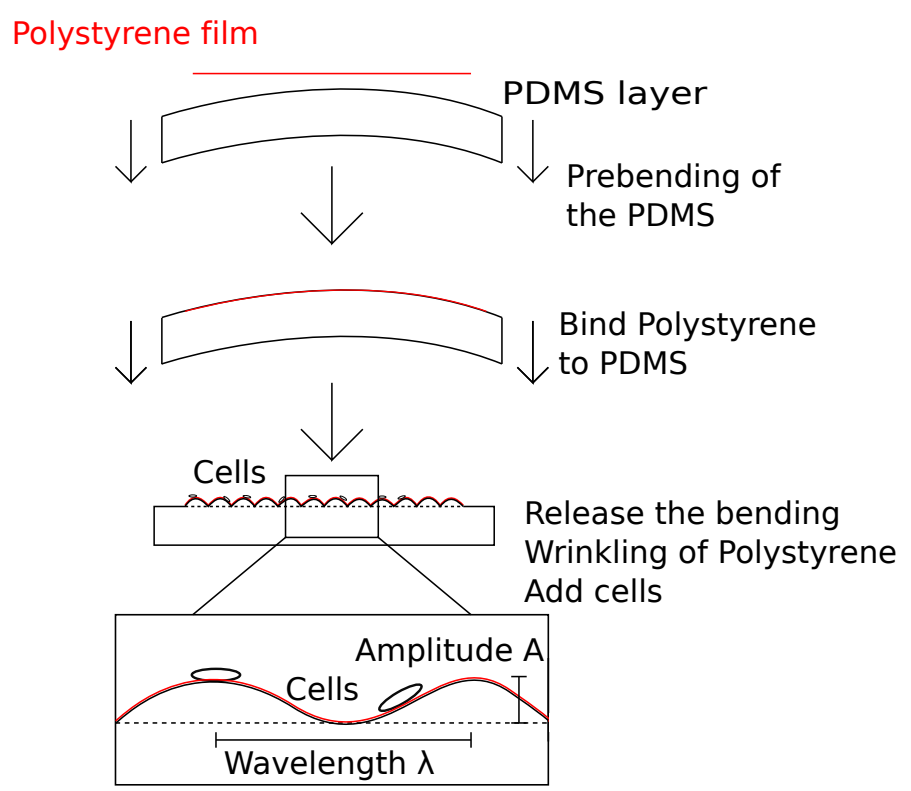

Figure 3.7: Sketch of the construction method of the wrinkled Curvotaxis device. The PDMS layer is first hold under tension. Afterwards the thin polystyrene film will be bound to the PDMS. After releasing the tension of the PDMS the wrinkling instability causes periodic waves.

foundation of stiffness $K$. Both dependencies are shown in the following equations:

$$
\begin{aligned}
& \lambda \propto\left(\frac{B}{K}\right)^{\frac{1}{4}}, \\
& A \propto\left(\frac{\Delta}{W}\right)^{\frac{1}{2}} \lambda .
\end{aligned}
$$

The resulting wrinkles range from scales of a wavelength of few micron to several hundred micron. Hence we obtain a cell compatible substrate with defined curvature, on that we can image tens to hundreds of cells. 


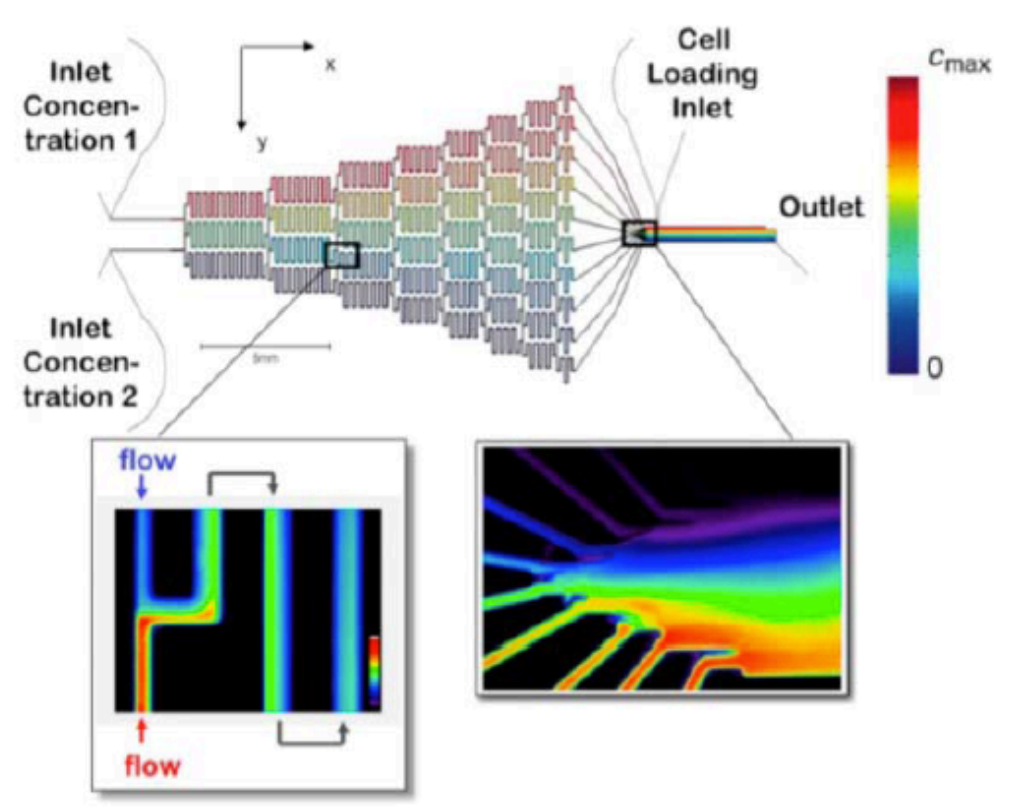

Figure 3.8: Sketch of the gradient mixer. Fluid with two different concentrations is filled in the two inlets. Due to diffusive mixing the fluid will be mixed in several steps until there are ten different concentrations that will be incorporated in the main channel. Hence the $D . d$. cells will be placed in a linear gradient of cAMP. Graph taken from (77) based on (71).

\subsubsection{Microfluidic Gradient Mixer}

For the chemotactical migration stimulation, we use a microfluidic diffusive mixer, as described by Song and colleagues (71), which is based on the work of Jeon and coworkers (41). With this gradient mixer, we achieve a well-controlled linear gradient of cAMP in an extended micro-channel. Hence it is a ideal tool to study chemotaxis in $D$. $d$. The cAMP concentrations $\left(c_{\max }=200 \mathrm{nM}\right.$, corresponding to $\Delta c=0.4 \frac{\mathrm{nM}}{\mu \mathrm{m}}$ ) we use are in the range maximal chemotactic velocity of the cells observed in the work of Song and colleagues (71) and consistently in the diploma thesis of Theves (77). In contrast to previous studies, we investigate the migration at the single cell level and not on the population level. 


\section{CHAPTER 4}

\section{Curvotaxis}

The forest soil amoebae $D . d$. live in a very complex three-dimensional environment. This is in contrast to the laboratory experiment which takes place on uniform flat glass surfaces. Hence we may ask the question, are these experiments the most appropriate way to understand the mechanism of amoeboid cell migration in their typical natural environment?

On our path to understanding of the full complexity of amoeboid cell motion, we leave the simple and nicely accessible, two-dimensional flat glass surfaces to arrive at the next step of complexity in introducing the third dimension of substrate complexity to cell migration by using a curved, yet still two-dimensional surface. A appropriate setup to study this question are optical fibers, as they are easy to get and to handle and they are curved in one direction while in the perpendicular direction their curvature is zero. We find that $D . d$. cells prefer to migrate in the curved direction of optical fibers. We call this anisotropic cell migration towards the curved direction "Curvotaxis". 
As further step towards higher complexity, we also introduce cell migration on wave-like structures. The cell behavior as well as the substrate are more complex, the cells migrate mostly in a direction in between the the curved and the non-curved direction.

In this chapter, we will start by introducing the aspects of curvature and the tools for analyzing the anisotropic cell migration. Then we will present the experimental results sorted by the following categories:

- Fluid flow direction

- Measurement chamber

Afterwards we analyze the dependencies of the curvotactic migration on the radius of curvature of the geometries. Finally, we will compare the hallmarks of curvotaxis on both substrates.

\subsection{Related research}

In recent research, the investigation of cell behavior in complex environments has increased. There are many approaches to address the question of more complex as well as more realistic experimental setups. Despite the fact that there are other setups where cells change their behavior due to a geometrical guidance from an environmental cue, curvotaxis can be distinguished from those effects. A close relation to other experiments is found in recent literature (4, 25, 26, 34, 55). In all these publications, $D . d$. cells are migrating on a more complex geometry than just a flat surface. Those substrates are different from our curvotaxis setup. In the case of $(4,34)$, the cells migrate on flat PDMS, but on the surface there are small 
PDMS pillars of the height $10-12 \mu \mathrm{m}$ and a diameter of $4 \mu \mathrm{m}$. Inside this array of micropillars, the cells are facing the flat surface of the bottom layer, as well as the curved surface of the pillars. These publications are concerned with cell migration as a function of different pillar densities, but Gorelashvili et al. also report that "cells in directed motion states sense the pillars as attractive topographic stimuli" (34). Although a fiber with radius of $2 \mu \mathrm{m}$ could not be used in our curvotaxis setup, this finding indicates that the curvotaxis effect may still be valid for smaller length scales. Their analysis shows that cells lacking myosin-II are not attracted to the pillars (4), which agrees nicely with our finding of cells lacking myosin-II activity not showing any curvotaxis.

A more confined experimental setup to investigate the influence of the topography on $D . d$. cell migration is shown in the joint work of the Beta and Losert groups in (55). In order to create a confined migration device that is similar to narrow interstitial spacings, cells may have to squeeze through, they designed a small linear microfluidic channel with height $20 \mu \mathrm{m}$ and width of $10 \mu \mathrm{m}$. In this way the symmetry-breaking of a single confined cell can be investigated, as it may only walk in on direction, either to the right or to the left. Nagel and his colleagues find that the migration mechanism is unaffected, as the pseudopod extension, even in this confined setup, shows still the typical alternating manner of splitting.

Another way to analyze migration in a complex environment is shown in (25), where $D . d$. cells can eventually migrate up a ramp and may come close to a cliff. At this cliff the cells mainly orient along the edge of the cliff and migrate towards the needle of the cliff. Hence again, the cells react to the complex environment in a distinct way. The Losert group investigates whether this migration is induced by contact guidance, which is known for other cell types to take place when the cells 
face tiny structures like nanometer-sized fibers. Hence they produced a grating that includes parallel nanoridges with a width of about $250 \mathrm{~nm}$ and a height of a few hundred nanometers. Those ridges posses a constant spacing that is varied. They found that most of the cells orient and migrate along these ridges, as it is supposely the mechanism relevant for contact guidance. Contact guidance is a phenomenon known from mammalian cells, as they migrate in vivo on an extracellular matrix or in experiments on fibers (27) or in 3D gels ( 62$)$, for instance made of collagen fibers. In these experiments the cells orient and migrate along these small structures. The mechanisms of contact guidance were already investigated in more detail (3). The BAR domain superfamily is presented to play a crucial part in the curvature sensing and membrane deformation on the nanometer scale (65). The member IBAR of this family was also found by the Faix group to have functions, like in filopodium formation and in cytokinesis in $D . d$. (51). Thus state-of-the-art knowledge indicates that these known mechanisms only describe curvature sensing in the range from several nanometer up to several hundreds of nanometer. Additional sensing of curvatures of several hundred micrometers by this mechanism can be excluded due to the size of IBAR. As the radii of curvature in our experimental studies range between $15 \mu \mathrm{m}$ and $150 \mu \mathrm{m}$ the mechanism of IBAR is not likely to describe this effect. 


\subsection{Curvature Definition and Analysis}

\section{Techniques}

In the overall scope of this thesis, curvature plays a crucial role both on the level of substrates as well as for the membrane geometry. Therefore we need to introduce the concept of curvature in the two-dimensional planar environment as well as in the three-dimensional environment.

\subsubsection{Two-Dimensional Line Curvature}

To relate curvature to chemotactic migration, biochemical signaling and cell shape, we use the line curvature of the cell membrane as a measure for cell contour. The two-dimensional line curvature of a mathematical curve is a relatively simple concept. The curvature is only dependent on the second derivatives.

\section{Definition:}

Let $c: I \rightarrow \mathbb{R}^{2}$ be a planar curve with a domain $I$, that is parameterized by the arc length. Then we shall call the function

$$
\kappa: I \rightarrow \mathbb{R}^{2}, \kappa(t):=\|\ddot{c}(t)\|
$$

the curvature of the curve $c$ at a time $t$.

In this thesis, this concept is needed to calculate the curvature of the two-dimensional projections of $D$. $d$. cells that are recorded with the confocal laser scanning microscope.

The result of this imaging technique is a two-dimensional image. Hence we have 


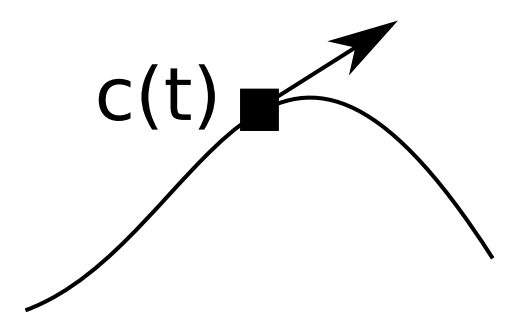

Figure 4.1: Sketch of a planar curve $c$ with its first derivative.

made a crude simplification by looking at a three-dimensional surface as a closed curve in two dimensions. However, up to this point, a more realistic description, as for instance three-dimensional image stacks of the cells, suffers from very poor temporal resolution. Hence using the curvature of a confocal plane projection of the cell membrane is the best measure we can rely on so far.

\subsubsection{Three-Dimensional Curvature}

While migrating in natural environments, as the forest soil and leaf litter, $D . d$. cells are sitting on a two-dimensional surface embedded in three-dimensional space and may be exposed to huge variety of possible curvatures. To characterize the curvature a cell is exposed to at a specific point in time we use the path along which the cell migrates and calculate the curvature of this path.

The concepts of curvature in three dimensions are more diverse than the twodimensional line curvature and need to be explained in more detail. A very important fact is that the curvature of a single point on a surface is not unique. This difference between the two-dimensional and three-dimensional cases have strong implications. A first important step is to distinguish between the possible concepts of curvature in three spatial dimensions. Firstly there is the straight forward extension of line curvature to three dimensions. Secondly we can investigate the curvature of a two- 

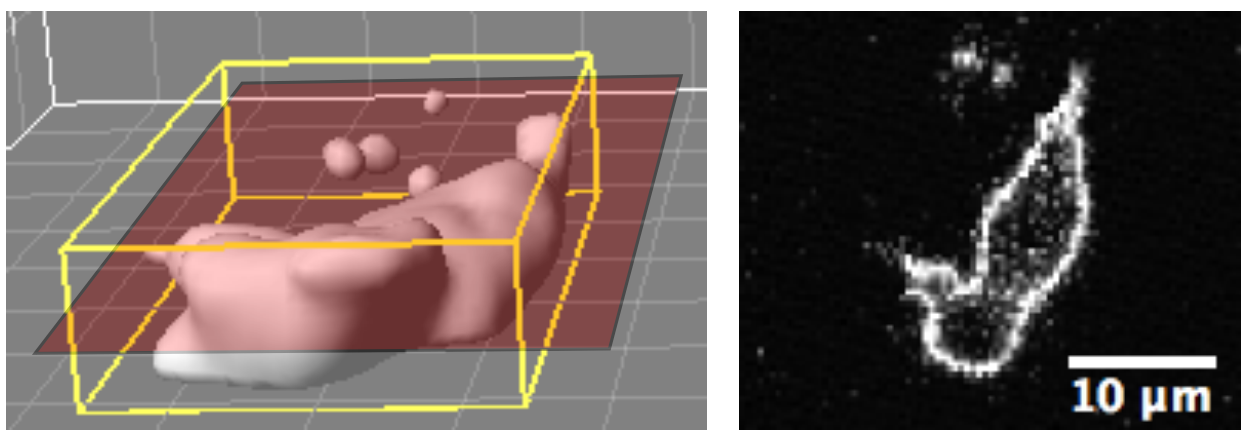

Figure 4.2: Using two-dimensional projection as measure for three-dimensional morphology. On the left-hand side, we can see a three-dimensional rendering of a $D . d$. cell labeled with a membrane associated label. This rendering is calculated from a stack of two-dimensional confocal fluorescence images. The red plane in the image corresponds to a projection of the cell in this plane, which is shown on the right-hand side.

dimensional smooth surface embedded in the three-dimensional space. To quantify the curvature of such spaces we need some more mathematical tools, which will not be explained in this thesis as it is of no practical use here. A deep mathematical introduction can be found in (즈). For such a surface you can find the two principal curvatures for any point on the surface and with simple arithmetic you can extract the Gauss curvature and the mean curvature of each point. As we are interested in curvature of a cell on the surface along its migration path the curvature of a space curve in three dimensions is a more suitable approach to our problem and we do not have to deal with the more complex three-dimensional concepts.

\section{Definition:}

Consider $c: I \rightarrow \mathbb{R}^{3}$ a spatial curve that is parameterized by the arc length. Then we will call the function

$$
\kappa: I \rightarrow \mathbb{R}^{3}, \kappa(t):=\|\ddot{c}(t)\|
$$

the curvature of curve $c$ at time $t$. 


\subsubsection{Projections and Lengths}

A novel challenge of cell visualization in three dimensions is the calculation of distances. Basically all microscopy techniques, including the different techniques introduced earlier in part 3.2.2, visualize two-dimensional sections of the imaged sample. Thus the recorded images of our experimental setup will always be the projection of the three-dimensional reality into the two-dimensional image. A visualization of this challenge can be seen in Figure 4.3. A cell that migrates along a perfect half-circle perpendicular to the fiber axis of the optical fiber with radius $r$, covers a distance of $d_{\text {real }}=\pi \cdot r$. In contrast, the distance we can measure on our microscopic image is $d_{\text {projected }}=2 \cdot r$. That trivially leads to the fact that the distance and hence also the velocity of the cell along its trajectory has to be corrected by the correction factor $c_{c o r r}=\frac{\pi}{2}$.

As a circle is a highly nonlinear geometric entity, this correction factor is only correct for trajectories of a full half-circle. If one has a trajectory with the projected length of $d_{\text {projected }}<2 \cdot r$ one will find infinite amount of correction factors. As an example, we show the traveled distances of three trajectories with a constant projected distance $d_{\text {projected }}=\frac{r}{2}$.

We start with the trajectory that starts on the top of curved surface and is shown in red in Figure 4.4. The end point is denoted with $x_{1}=\frac{r}{2}$. 


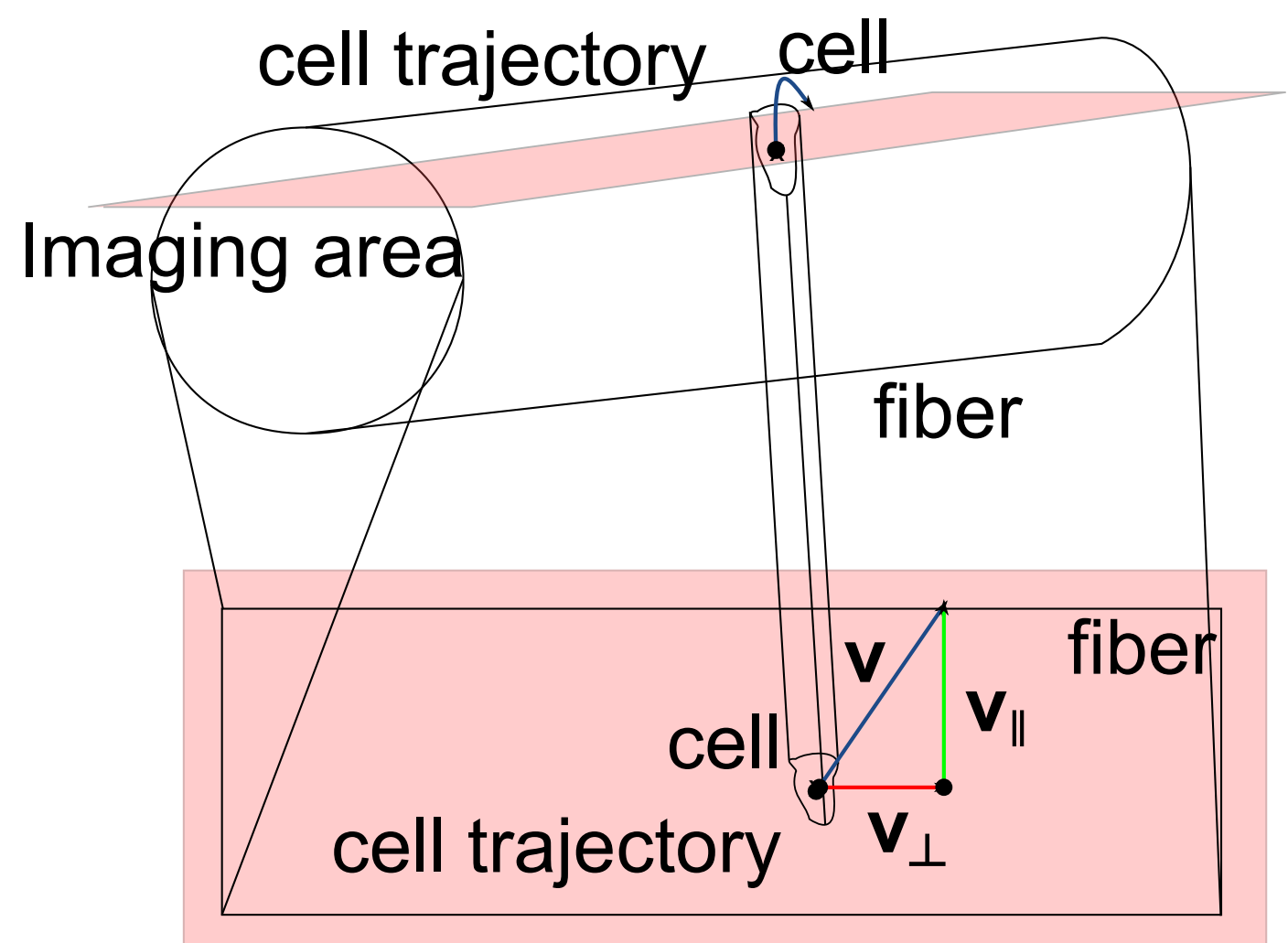

\section{Imaging area}

Figure 4.3: Sketch of projection from three-dimensional fiber to two-dimensional microscope image. The red plane represents the projected plane that is visible in the lower part of the figure, which we call Imaging area. The cell trajectory shown in blue can be seen in the upper part and its projection is the straight blue line in the lower part. The velocity can be decomposed into a component parallel to the curvature direction $v_{\|}$and another component perpendicular to it $v_{\perp}$. 


$$
\begin{aligned}
d_{\text {projected,red, } 1} & =\frac{r}{2}, x_{1}=\frac{r}{2} \\
\Rightarrow y_{1} & =\sqrt{r^{2}-x_{1}^{2}}=\sqrt{r^{2}-\frac{r^{2}}{4}}=\sqrt{3} \cdot \frac{r}{2} \\
\text { As } \tan \phi_{1} & =\frac{x_{1}}{y_{1}} \Rightarrow \phi_{1}=\tan ^{-1}\left(\frac{x_{1}}{y_{1}}\right)=\tan ^{-1}\left(\frac{\frac{r}{2}}{\sqrt{3} \frac{r}{2}}\right)=\tan ^{-1}\left(\frac{1}{\sqrt{3}}\right)=\frac{\pi}{6}(4.3) \\
\Rightarrow d_{\text {real }} & =2 \pi r \cdot \frac{\phi_{1}}{2 \pi}=\frac{\pi \cdot r}{6} \approx 0.524 \cdot r>\frac{r}{2} \\
c_{\text {corr }, 1} & =\frac{d_{\text {real }, 1}}{d_{\text {projected }, 1}}=\frac{\frac{\pi r}{6}}{\frac{r}{2}} \approx 1.0472
\end{aligned}
$$

For the second trajectory we use the endpoint of the first trajectory as the starting point $x_{1}=\frac{r}{2}$. We choose the maximal position of the fiber to be the end point of the trajectory $x_{2}=r$.

$$
d_{\text {projected }, \text { blue }, 2}=\frac{r}{2}, x_{2}=r
$$

As easily seen in Figure 4.4, the angle $\phi_{2}$ is strongly coupled to $\phi_{1}$ via $\phi_{1}+\phi_{2}=\frac{\pi}{2}$

$$
\begin{aligned}
\Rightarrow \phi_{2} & =\frac{\pi}{2}-\frac{\pi}{6}=\frac{\pi}{3} \\
\Rightarrow d_{\text {real }} & =2 \pi r \cdot \frac{\phi_{2}}{2 \pi}=\frac{\pi \cdot r}{3} \approx 1.047 \cdot r \gg \frac{r}{2} \\
c_{\text {corr }, 2} & =\frac{d_{\text {real }, 2}}{d_{\text {projected }, 2}}=\frac{\frac{\pi r}{3}}{\frac{r}{2}} \approx 2.0944
\end{aligned}
$$

The third trajectory passes the top of the curved substrate. The easiest way to calculate the angle $\phi_{3}$ and respectively the real travelled distance $d_{\text {real,3 }}$ is by dividing the path into the two symmetric parts with $d_{\text {projected }, 3}=\frac{r}{4}$ and treat them analogously to the first case. 


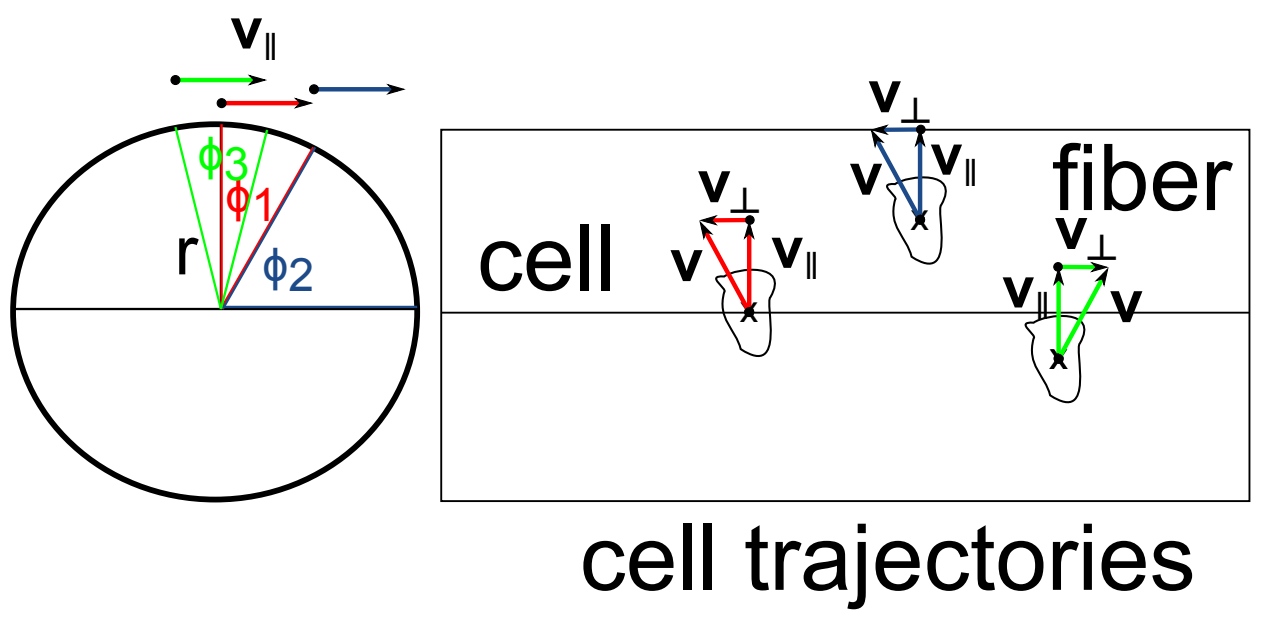

Figure 4.4: Sketch of three different exemplary trajectories with identical $d_{\text {projection }}$. On the projected micrographs all lengths in y-direction are equal, even though the actual migrated distances on the optical fiber differ strongly. The real traveled distances $d_{\text {real }}$ are segments of a circle, and therefore it is necessary to calculate the angle $\phi$ to determine those distances.

$$
\begin{array}{r}
d_{\text {projected,green }, 3}=\frac{r}{2}, x_{3}=\frac{r}{4}(4.10) \\
\Rightarrow y_{3}=\sqrt{r^{2}-x_{3}^{2}}=\sqrt{r^{2}-\frac{r^{2}}{16}}=\sqrt{15} \cdot \frac{r}{4}(4.11) \\
\text { As } \tan \phi_{1}=\frac{x_{1}}{y_{1}} \Rightarrow \frac{\phi_{3}}{2}=\tan ^{-1}\left(\frac{x_{3}}{y_{3}}\right)=\tan ^{-1}\left(\frac{\frac{r}{4}}{\sqrt{15} \frac{r}{4}}\right)=\tan ^{-1}\left(\frac{1}{\sqrt{15}}\right) \approx 0.2527(4.12) \\
\Rightarrow d_{\text {real }}=2 \pi r \cdot \frac{\phi_{3}}{2 \pi} \approx 0.505 \cdot r>\frac{r}{2} c_{\text {corr }, 3}=\frac{d_{\text {real }, 3}}{d_{\text {projected }, 3}} \approx \frac{0.505 \cdot r}{\frac{r}{2}} \approx 1.01(4.13)
\end{array}
$$

In summary this means that depending on the cell position, the difference of the traveled distance may be very small. In the third case it is as few as $1 \%$, as well as intermediate as it is in the first case about $5 \%$, and it may also be huge as in the second case more than $109 \%$. Therefore, we need a way to correct for these strong differences. 


\subsubsection{Vector Decomposition}

We decompose the displacement in a component that is parallel to the curved direction and another component that is parallel to the flat direction, the axis of the optical fiber, or in other words, perpendicular to the curved direction.

The fiber is a three-dimensional object with a two-dimensional surface on which the cells can migrate. Mathematically speaking, the cells migrate on two-dimensional surfaces embedded in the three spatial dimensions. The principle curvatures of the

surface are $\frac{1}{R_{\text {fiber }}}$ and zero. Therefore the maximal curvature is $\frac{1}{R_{f i b e r}}$ and this is the curvature the cells "feel" if they migrate tangentially on the cylinder. Along a straight path the curvature is always constant.

\subsubsection{Special Case for Wrinkles}

In the case of the sinusoidal surface created by the wrinkling instability of thin polystyrene film bound to PDMS used in the present study, the curvature changes depending on the position on the substrate and the direction in which the cells migrate.

In the case of the wrinkled substrate, the analysis of the traveled distance differs slightly as the surface is sinusoidal. Hence the calculation changes, but the general property that the migrated distance is larger than the projected value is conserved.

We assume that we can approximate the surface $S$ of the wrinkled substrate by a sinusoidal waveform. We can write it as

$$
S:(x, y) \longmapsto(x, y, A \sin (k \cdot y+\phi)) .
$$


To achieve the same vector decomposition as for the fibers on the wrinkled substrates, we will calculate the actual migrated distance extracting the collected projection from the micrograph. Here we cannot use the trivial calculation of the fraction of a full circle to extract the real migrated distance as arc length of the migrated curve. A more reasonable way is to calculate the arc length as learned in the vector analysis for a function $f(\varphi)$ sfrom position $\varphi_{1}$ to position $\varphi_{2}$ :

$$
\begin{aligned}
s\left(\varphi_{1}, \varphi_{2}\right) & =\int_{\varphi_{1}}^{\varphi_{2}} \sqrt{1+f^{\prime}(\varphi)^{2}} \mathrm{~d} \varphi \\
& =\int_{\varphi_{1}}^{\varphi_{2}} \sqrt{1+(A \cos (\varphi))^{2}} \mathrm{~d} \varphi .
\end{aligned}
$$

\subsubsection{Curvotactic Anisotropy Parameter}

For $D . d$. chemotaxis, a measure of how well cells migrate in a the chemical gradient is the chemotactic efficiency that is defined by the velocity in gradient direction $v_{\text {grad }}$ divided by the total velocity $v$.

$$
C E=\frac{\left\langle v_{\text {grad }}\right\rangle}{\langle v\rangle}
$$

In this work we define a parameter to measure the anisotropy of the migration in a complex geometry. As we are interested in the different behaviour of the cells according to the complex environment, we define the Curvotactic Anisotropy Parameter $(C A P)$ as the absolute value of the velocity $\left|v_{\|}\right|$in the curved direction 


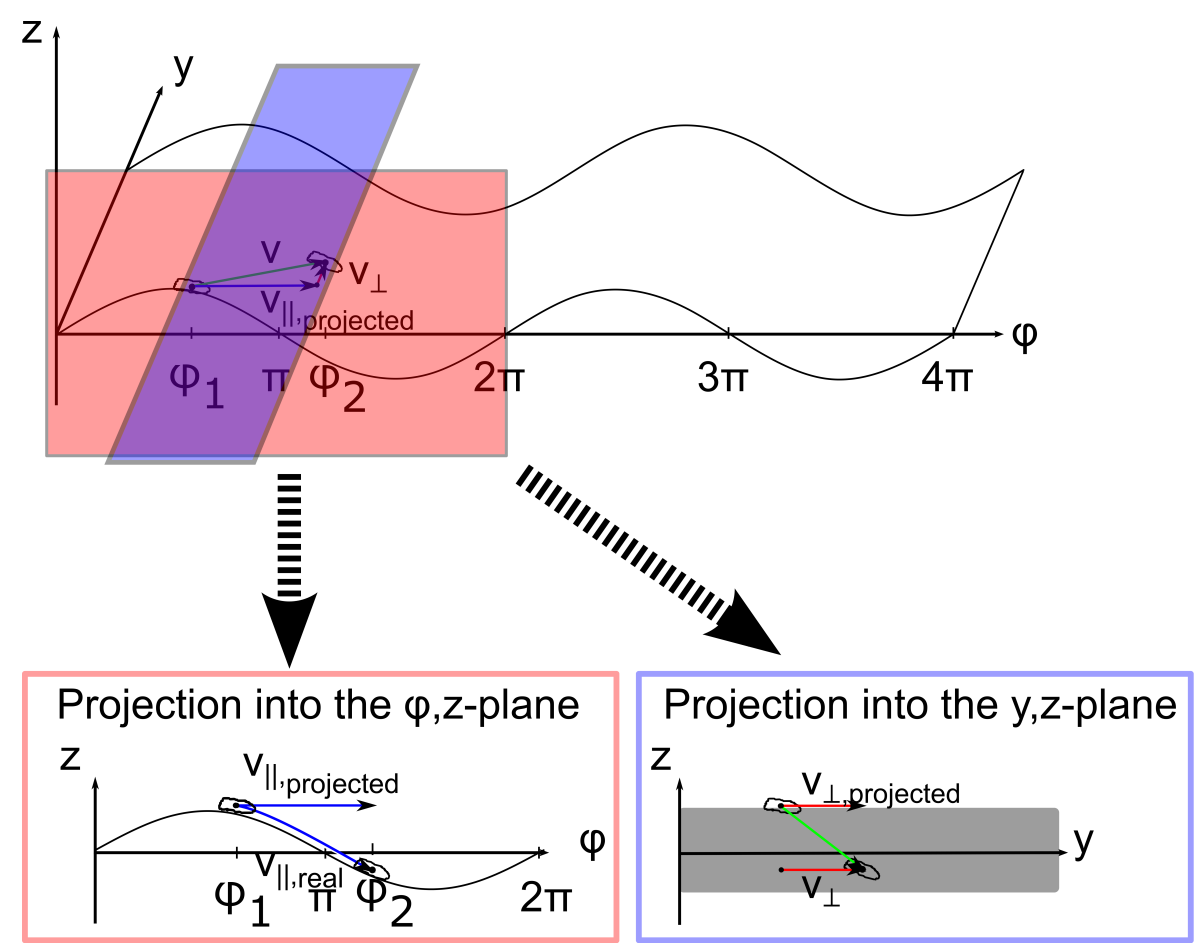

Figure 4.5: Sketch of a migrating cell and its trajectory in direction perpendicular to the wrinkles. The projection of the migrated trajectory into the $\phi, z$-plane has a different length than the actual curve. Hence the length of the trajectory has to be corrected accordingly via the arc length of the function the trajectory. The projection into the $y, z$-plane does not have a different length than the real curve, hence there is no need for a correction.

divided by the absolute value of the velocity in the perpendicular direction $\left|v_{\perp}\right|$ :

$$
C A P=\frac{\left\langle\left|v_{\|}\right|\right\rangle}{\left\langle\left|v_{\perp}\right|\right\rangle}
$$

In contrast, to the chemotactic efficiency $C E$, where the target set is $[-1,1]$, the target set of the curvotactic efficiency is $[0, \infty)$. Hence a value of one for the curvotactic anisotropy parameter corresponds to a setting where the velocity is the same in both directions e.g. a random walk. A higher the $C A P$ corresponds to the faster cells migration in the direction of the curvature. 


\subsubsection{PCA-Characterization of Trajectories with Convex}

\section{Hulls}

Another measure to describe the anisotropy of the cell migration is to use the convex hull of each trajectory; such principal component analysis (PCA) have been used to analyze $D . d$. shape in various gradients of cAMP (79).

For this reason we construct a convex hull around a trajectory. Hence we choose all points of the trajectory such that all convex combinations of the points contained in the trajectory are included in the polygon of the convex hull. As an illustration of the advantages using the convex hull analysis, see Figure 4.6. In mathematical terms, we have to introduce the terms convex set and convex hull as:

Definition: A set $S$ is convex, if for all vectors $x$ and $y$ in $S$ and $0 \leq \lambda \leq 1$, the point $t=\lambda x+(1-\lambda) y$ is also in $S$.

$$
S \subset V=\mathbb{R}^{2} \text { convex if } \forall x, y \in S \Rightarrow t=\lambda x+(1-\lambda) y \in S
$$

Definition: The convex hull $\operatorname{co}(S)$ of a set $S$ contained in a vector space $V$ is defined as the intersection of all convex sets $U$ in $V$ containing $S$.

$$
\operatorname{co}(S)=\bigcap_{\substack{U \text { convex } \\ S \subset U \subset V}} U
$$

Besides this set theoretical definition one can also write a more geometrical one 


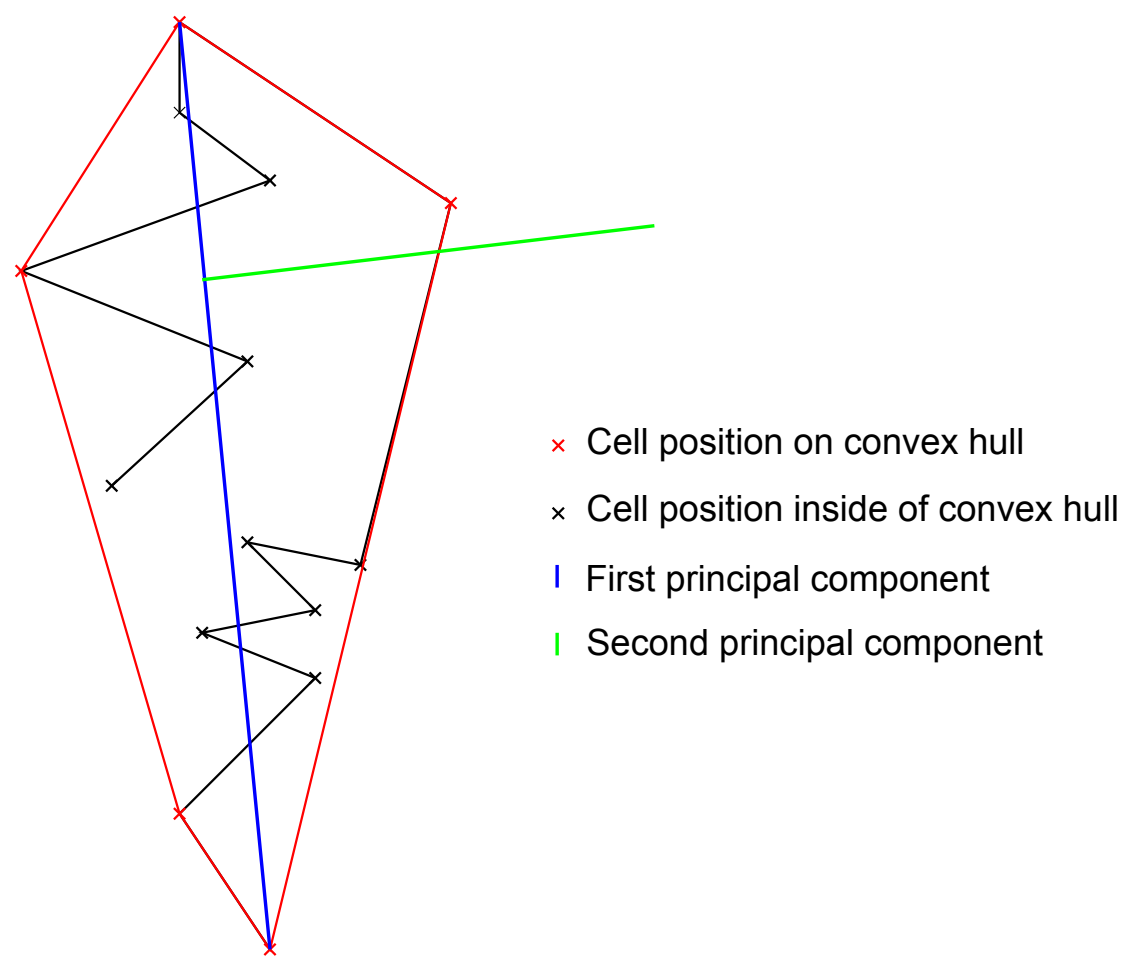

Figure 4.6: Sketch of a trajectory and in red included the convex hull and all the points of the hull. The blue line denotes for the first principal component. It has a distinct direction and length. The second component is shown in green. It is perpendicular to the first component and it is shorter then the first component.

using the convex combinations of the elements of $S$.

$$
\operatorname{co}(S)=\left\{\sum_{i=1}^{n} \alpha_{i} s_{i} \mid s_{i} \in S, n \in N, \sum_{i=1}^{n} \alpha_{i}=1, \alpha_{i} \geq 0\right\}
$$

It is clearly visible in Figure 4.6 that the convex hull of a set of points in two dimensions defines the polygon surrounding all points and including every line segment between two of the points crossed while migrating.

After we have constructed the convex hull, we can use its geometry to analyze the properties of the cell trajectory that defines it. We use the principal component 
analysis of the constructed two-dimensional polygon. The results of this analysis are the two vectors corresponding to the first and second principal components. With the two vectors, we can on the one hand classify the different convex hulls into elongated hulls where the first principal component is much bigger than the second and amorph hulls where both principal components have about the same length. If a cell trajectory leads to an elongated convex hull, the cell had one direction in which it explored much more distance than in all other directions. As visible in Figure 4.6, the cell may as well walk back and forth in this direction.

On the other hand we extract the directionality of the convex hull and know precisely the principal direction. That means that we obtain a new measure to quantify the anisotropy of cell migration. In particular, the effect of single small steps decreases dramatically in this analysis.

Using a global measure of the cell migration like the convex hull, minimizes the influence of the error due to manually tracking the $D$. $d$. cells.

\subsubsection{Atomic Force Microscopy for Substrate Analysis}

As there are several studies that report on the influence of nano-sized patterns on the migration of motile cells, we investigated whether there is any nanostructure on either of the two presented substrates. Hence we did experiments with Atomic Force Microscopy to characterize the properties of the substrates in the nanometer regime. In case of the wrinkled substrates, there is an important question that the Atomic Force Microscope (AFM) is able to answer. The height of the sinusoidal wave-like structure can be measured by the AFM experiment.

The results of the AFM experiment on the cylinders are shown in Figure 4.7. The 
graph on the left shows an image of $40 \mu \mathrm{m} \times 40 \mu \mathrm{m}$ of imaging mode of the AFM. The cylinder axis (cylinder radius 80 microm) is placed at around $20 \mu \mathrm{m}$ and the height decreases both to the right and to the left. There is no additional structure besides the change due to the curvature of the cylinder. If one calculates the height difference using the assumption that the fiber is right in the center, this value is $2.3 \mu \mathrm{m}$ and in perfect agreement of the shown result of the graph. On the right hand side graph of Figure 4.7 the deflection is shown. A structured nanometer-sized pattern is not found. Therefore we can use the cylinders to study cell migration on curved structures without worrying about an influence of contact guidance.
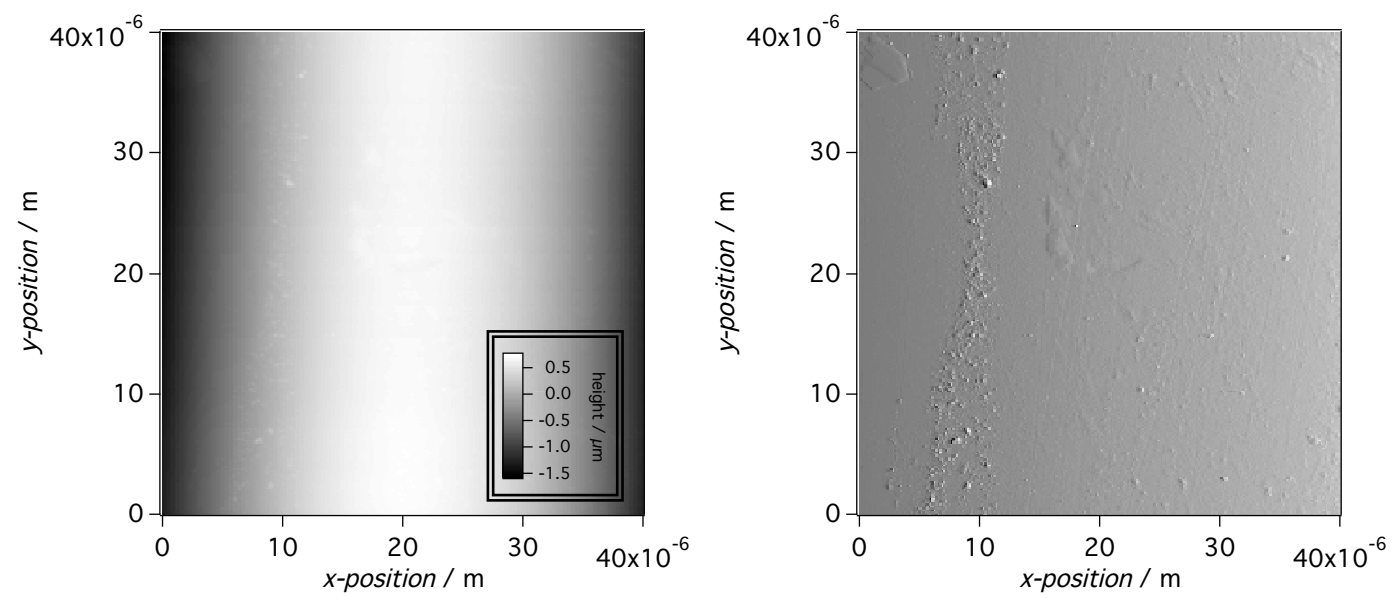

Figure 4.7: Experimental results of AFM measurements. The graph on the left hand side shows the height profile of the optical fiber measured with the AFM. At a position of $20 \mu \mathrm{m}$ there is the maximum of the fiber and to the right and to the left the height decreases. The graph on the right hand side shows the deflection of the cantilever as a gray scale. It describes the changes of the parameters of the control circuit and is a measure for roughness of the sample. On this image there is no structure visible, besides some dirt, especially no nanometer-sized structure in curvature direction which could influence the cells via contact guidance.

An image of the height profile of a wrinkled substrate measured with atomic force microscopy is shown in Figure 4.8. The wrinkles possess a wave length of $80 \mu \mathrm{m}$ and 


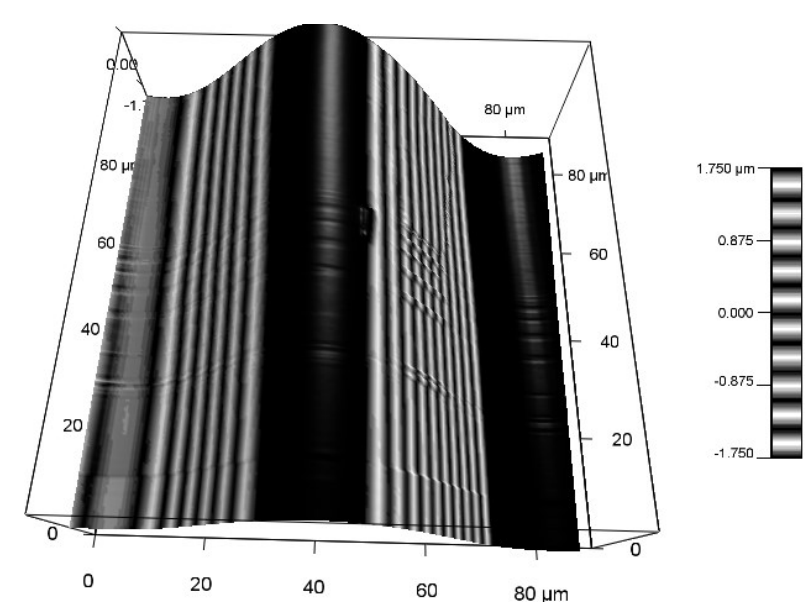

Figure 4.8: Height profile of a single wrinkle wave. The color code uses black and white stripes for visualizing height differences. The amplitude of the wave is $1.5 \mu \mathrm{m}$ and its wave length is $80 \mu \mathrm{m}$.

a height of $1.5 \mu \mathrm{m}$. The black and white stripes on the picture are the color code to visualize the height differences. The few small stripes in the middle of the substrate perpendicular to the wrinkles are artefacts of the cantilever due to the controlling feedback.

In Figure 4.9, we show an overlay of the the height profile (small inlay) and the top view bright field image, where one can see the wrinkled substrate as well as the cantilever. In this way, we can correlate the geometrical property of the substrate with the bright field image.

As a result, we could not find any evidence that there is any nanometer-sized pattern on either of the substrates. We conclude that the process investigated in this thesis is not influenced by the process known as contact guidance. 


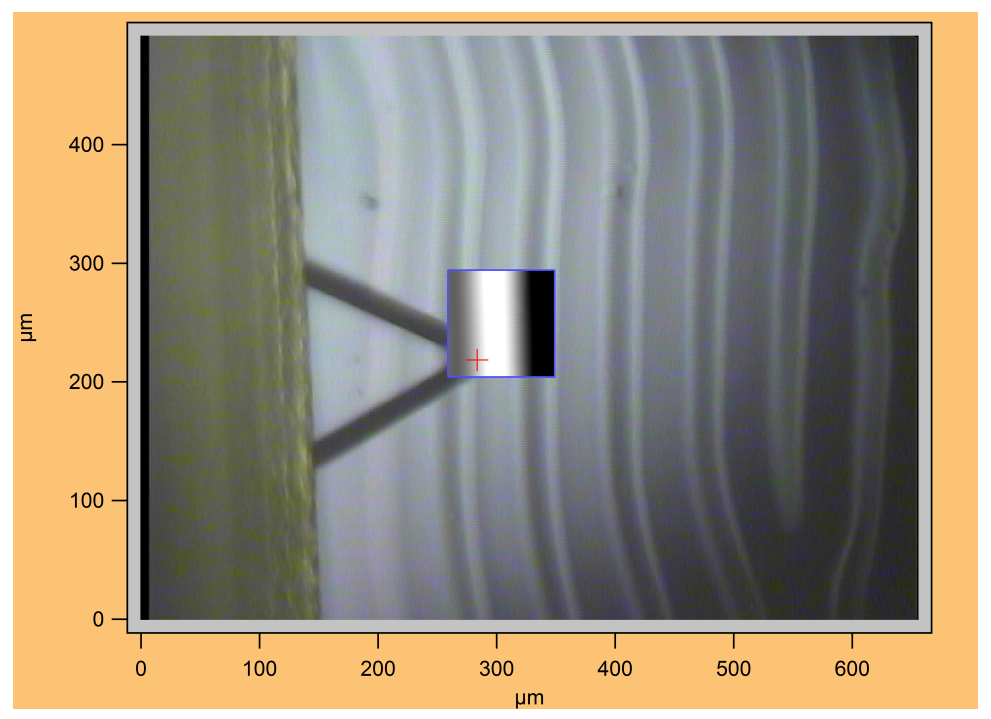

Figure 4.9: Overlay of the height profile (black and white inlay) and the top view bright field image with a gray scale in arbitrary units. The cantilever of the AFM is visible on the left hand side. The wrinkled structure of the substrate is also visible. In the inlay, the cantilever position is marked by the red cross. The height profile inside the inlay is color-coded with white for the maximal height and black for the minimal height. The image is the same as in Figure 4.8.

\subsection{Curvotaxis on Cylinders}

The results of curvotactic migration of $D$. $d$. cells on cylinders, i.e. optical fibers are shown in this section. We used different fiber diameters between $28 \mu \mathrm{m}$ and $260 \mu \mathrm{m}$ to investigate the influence of the curvature on the migration of the cells.

In Figure 4.10 a typical DIC micrograph of the a single experiment is shown with $D$. $d$. cells on the optical fiber including the cell trajectories. At a first glance, it is already apparent that the cells seem not to move isotropically. This effect is even stronger than the impression that is visible by eye, as the migrated distance in the direction parallel to the curvature is always larger than the distance we can measure in our projected micrograph, as shown in part 4.2.3. This impression will 
be confirmed in the following section by a rigorous analysis. We then divide the results in different categories for different cell lines and different experimental setups. Finally, we will show the combined data from all categories.

\subsubsection{Representative Single Experiment}

In this section, we will present a single exemplary experiment, including the details of the results and the analysis to emphasize on properties as the cell-to-cell variability.
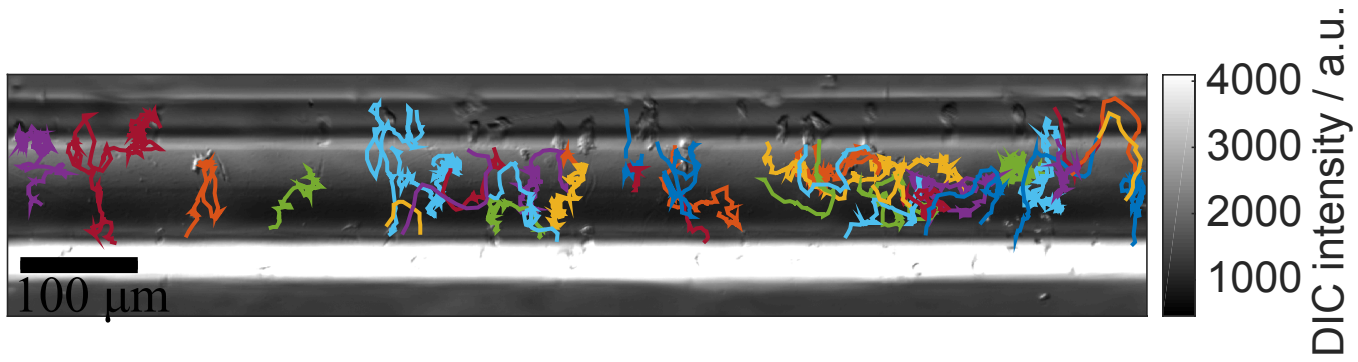

Figure 4.10: Overlay of the first micrograph of a time series of migrating cells and the corresponding 43 trajectories extracted from this series. We used AX2 cells labeled with LimE-GFP. The diameter of the optical fiber is $160 \mu \mathrm{m}$ and the images recorded with an Olympus confocal laser scanning microscope Fluoview 1000 in the DIC mode. The different colours of the trajectories are used to distinguish between individual trajectories. Gray scale represents the DIC intensity in a.u., while the black scale bar corresponds $100 \mu \mathrm{m}$.

This example stems from a microfluidic flow experiment with AX2 cells including the filamentous actin marker LimE-GFP. The raw data in Figure 4.10 show the initial image of the image series in gray scale. All 43 trajectories of migrating cells are shown overlaying in different colors to distinguish between individual trajectories more easily. The trajectories are noisy and more oriented in the curved direction.

Figure 4.11 shows a histogram of the migration angle of the cells. It includes all 


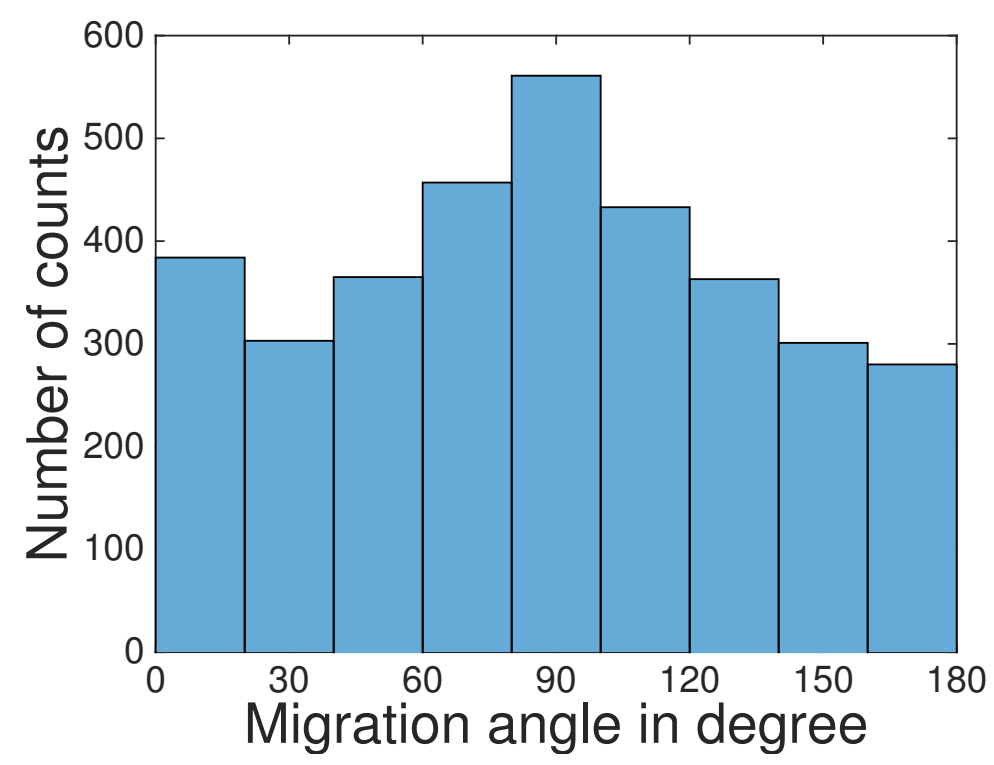

Figure 4.11: Histogram of migration angles of 42 motile $D . d$. cells on the fiber shown in Figure 4.10 for all steps of the trajectories that migrate a minimum distance of $10 \mu \mathrm{m}$. A very distinct peak at a migration direction of $90^{\circ}$ is found.

migration angles of all trajectories that are found for motile cells. Being motile is defined as migrating at least one cell length $(10 \mu \mathrm{m})$. Thus we exclude cells that do not move, being unhealthy, or even dead. We find cell trajectories durations ranging from length from $12.5 \mathrm{~min}$ to $120 \mathrm{~min}$, what was the total duration of the experiment. We find the base line of the histogram at around 300 counts with a clear peak at a migration angle of $90^{\circ}$ including more than 560 counts. Moreover, the bins closest to the peak are substantially higher than the base line. Hence most of the migrational steps are in the curved direction.

Another way to confirm the anisotropy of the $D . d$. cell migration on the curved substrate is the convex hull analysis introduced in section 4.2.6. The results are shown in Figure 4.12 as a histogram of the direction of the first principal component of the elongated trajectories. Here we exclude all trajectories where the main component 


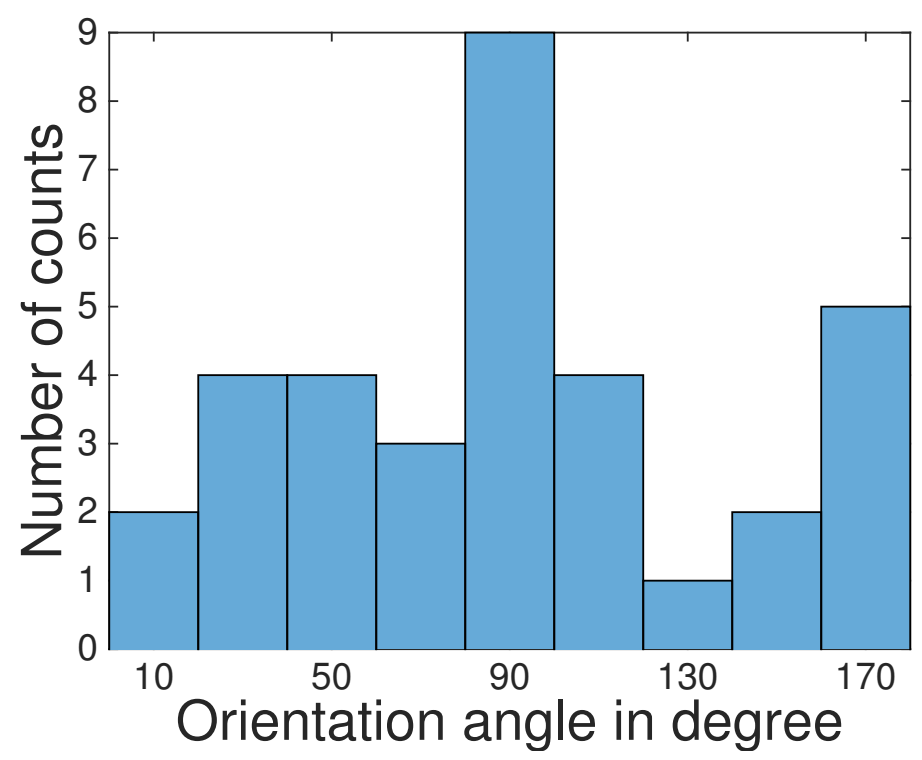

Figure 4.12: Histogram showing the orientation of 35 motile $D . d$. cell trajectories on an optical fiber which are elongated (absolute value of first component twice as high as absolute value of second component). The orientation is defined by the direction of the convex hull compared to the axis of the optical fiber, where $0^{\circ}$ corresponds $0^{\circ}$ of the migration angles, or in other words along the fiber axis. The peak of the orientation of the trajectories is at $90^{\circ}$ corresponding to the curved direction.

is less than two time the smaller component. As the number of trajectories is much smaller than the number of migration steps, the sampling of the histogram is not as good as in Figure 4.11. The orientation angle of a complete trajectory is, however, a much more robust way to quantify the anisotropy. At the level of up to $150 \mu \mathrm{m}$ migrated distance the error made by manually tracking the cells is negligible compared to the same error made at a single step that may be on the order of one micrometer. The histogram in Figure 4.12 shows a clear peak at an orientation angle of $90^{\circ}$, corresponding perfectly to the result for the migration angle. We additionally investigate the dependence of the magnitude of the migrational velocity 
on the migration direction. The result for the exemplary experiment is shown in Figure 4.13. We binned the data as in the orientation histograms in Figure 4.11 and 4.12 and show the mean and the standard deviations of each bin. In direction of the curvature, the main velocity of the cells is maximal and more than $40 \%$ higher than as for the migration along the flat direction. Finally we can investigate the velocity parallel and perpendicular to the curvature and compare them to the total velocity, and thus calculating the curvotactic efficiency introduced earlier (Section 4.2.5). For this experiment, we find the following average velocities.

$$
\begin{aligned}
<\left|v_{\perp}\right|> & =3.5 \frac{\mu \mathrm{m}}{\mathrm{s}} \\
<\left|v_{\|}\right|> & =4.7 \frac{\mu \mathrm{m}}{\mathrm{s}} \\
<|v|> & =6.6 \frac{\mu \mathrm{m}}{\mathrm{s}} \\
\Rightarrow C A P & =1.36
\end{aligned}
$$

The velocity parallel to the curvature $v_{\|}$is $36 \%$ higher than the perpendicular velocity $v_{\perp}$. This leads to a curvotactic anisotropy parameter of $C A P=1.36$.

All introduced measures thus confirm the anisotropy of the migration on a curved substrate.

\subsubsection{Influence of the Experimental Setup}

In this section, we look at different experimental setups with their different properties to apply the application of fluid flow relative and to thereby exclude the influence of chemotactic signaling due to cells secreting cAMP.

The experimental setup contains four different specific designs. In the simplest 


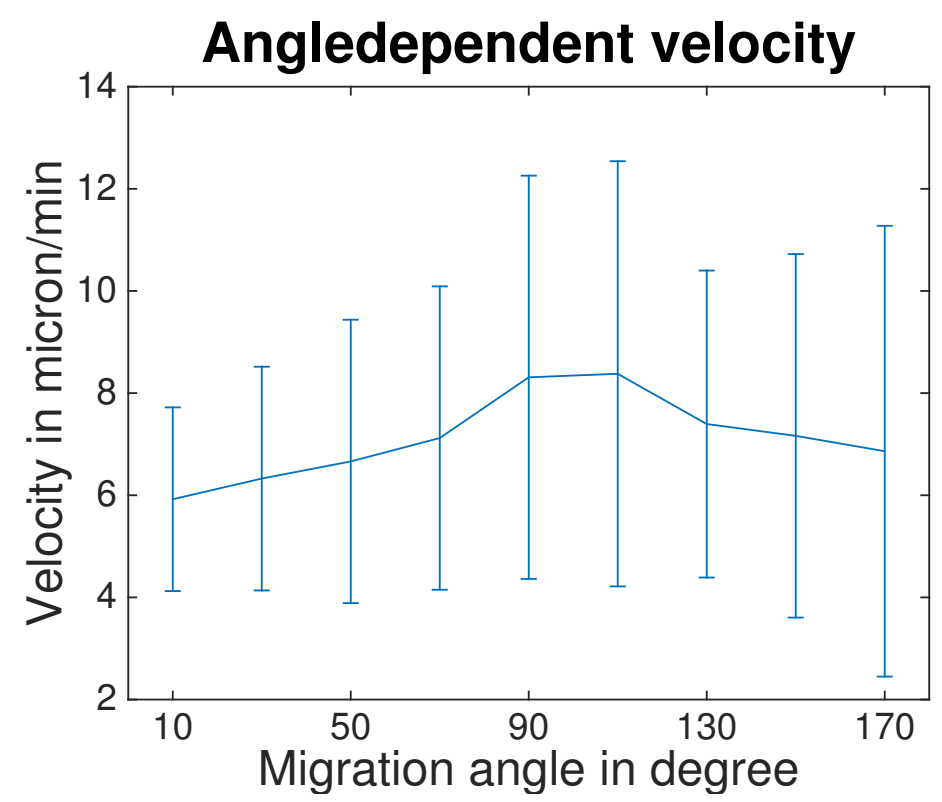

Figure 4.13: This plot shows the migration velocity of $D$. $d$. cells dependent on the migration direction of the cells for the exemplary microfluidic experiment. It shows that the cells that migrate in the direction parallel to the curvature are quicker than cells that migrate in different directions.

possible setup, we placed the optical fibers in a petri dish, in which we cannot control the fluid flow. This setup is very easy to handle. The drawback is that the cells might communicate with each other by cAMP signaling. To address this, we devised alternate experimental setups, which contain a controlled fluid flow to remove cAMP and allow to distinguish between the mechanisms due to chemotaxis and curvotaxis. One way to achieve a fluid flow around the fiber is to place it in a microfluidic channel. To achieve that, we need to use a channel that is bigger than the fiber diameter. The only way to place the fiber reasonably well in the microfluidic channel, is to align it with the microchannel. Another possible design is to place it in a perfusion chamber (described in section 3.2.3.1). This design allows to have the flow either parallel or perpendicular to the fiber axis. A drawback of placing the fiber parallel to the fluid flow is that cAMP produced by cells upstream in the 
device may influence cells downstream. This can be prevented by positioning the fiber perpendicular to the fluid flow. By having fluid transport around the fiber and by not imaging of cells downstream in the device, we can exclude the influence of chemotaxis.

We investigated different fluorescently labeled cell lines, namely RBD-GFP, LimEGFP, cAR1-GFP and cytosolic GFP HG-1692. Due to the imaging through the optical fiber, it is difficult to obtain clear fluorescent images. The fluorescence intensity we could gather for each of the cell lines was not sufficient to get an image where individual cells could be visualized. Therefore, the DIC module of the Olympus microscope software FV1000 was used to image the cell migration.

We extract the cell trajectories from the raw data manually as it is more stable and reliable than automated tracking due to the noisiness of the data.

As in the preceding section (4.3.1), we will show the four different measures of anisotropy, namely migration direction of cells, orientation of trajectories, angle dependent velocity and curvotactic efficiency. The different measures will be combined for all experimental setups in individual figures.

We begin with the plot of histograms of the migration angles corresponding to all four different experimental setups in Figure 4.14. In these histograms, we plot all migration steps of all migrating cells for the different categories. This means that we first divide all cells into migrating and non-migrating. We defined migrating cells as cells that migrate at least a distance of $10 \mu \mathrm{m}$ during their whole trajectory. As $D . d$. cells migrate in chemotactic gradients with an average velocity of around $10 \frac{\mu \mathrm{m}}{\mathrm{min}}$, we only exclude very slow or dead cells. In this analysis, the fraction of non-migrating cells was as small as $6 \%$. Additionally, we only plot the steps where the cells actually move. If a migrating cells is not moving from one timestep to the next, we do not use 


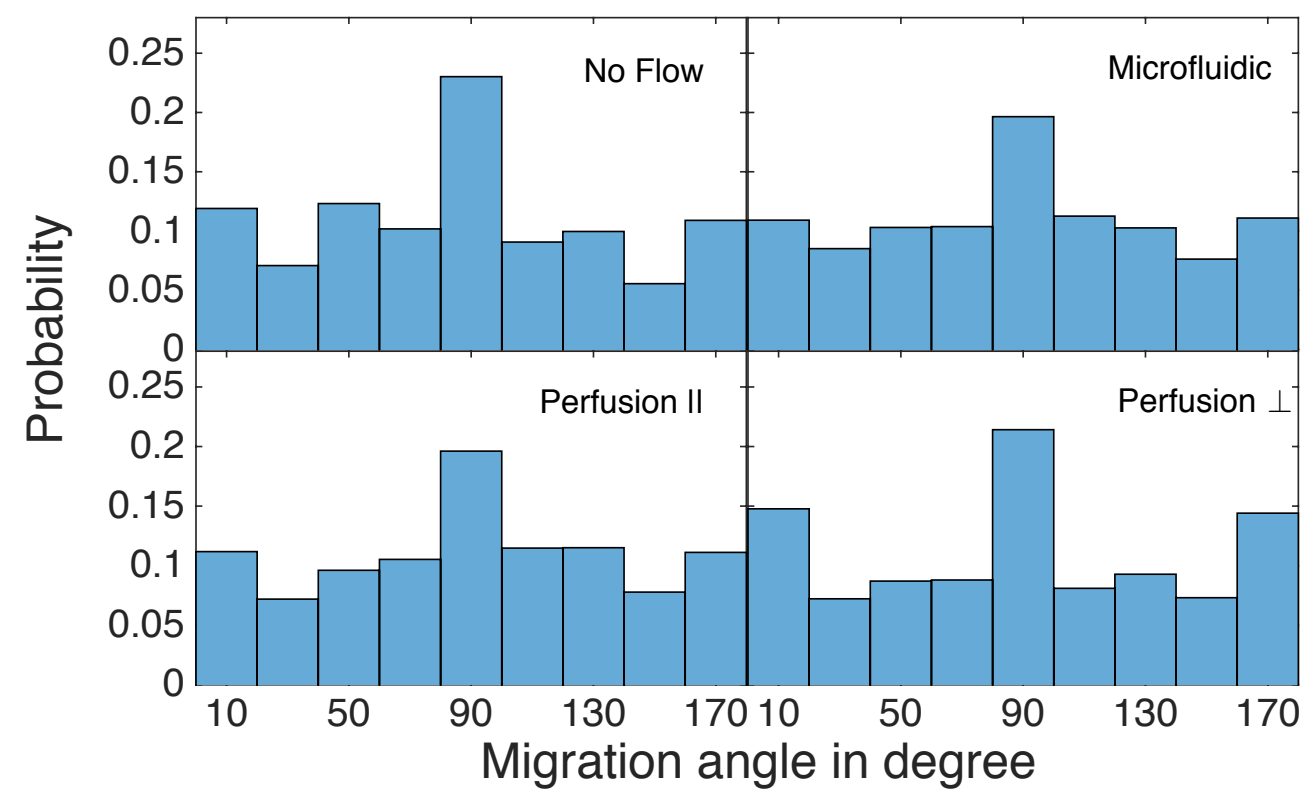

Figure 4.14: Migration angle histogram for all experimental setups. All migration step for cells that migrate at least 10 micron are used for the histogram. All four histograms show a clear peak at the migration angle of $90^{\circ}$. There are basically no differences between the histograms.

this step for the migration angle histogram. All histograms show a clear peak at $90^{\circ}$, which indicates a migrations along the y-direction or in other words the curvature direction. For all cases the peaks are for all cases at least $60 \%$ higher than the second largest peak and more than twice as high as the average of all other peaks. Thus we found an anisotropy of the cell migration in curvature direction.

Now we show, in Figure 4.15, the directionality of the convex hulls for the different experimental setups. The convex hulls were analyzed by a principal component analysis (PCA) as described in section 4.2.6. The two parameters that describe the two-dimensional shape of the convex hull in terms of a PCA are the orientation and the elongation. The components of a two-dimensional shape are two vectors $\mathbf{p}_{1}, \mathbf{p}_{2}$ which are orthogonal to each other: $\mathbf{p}_{1} \perp \mathbf{p}_{2}$. Therefore we can use the fraction of 


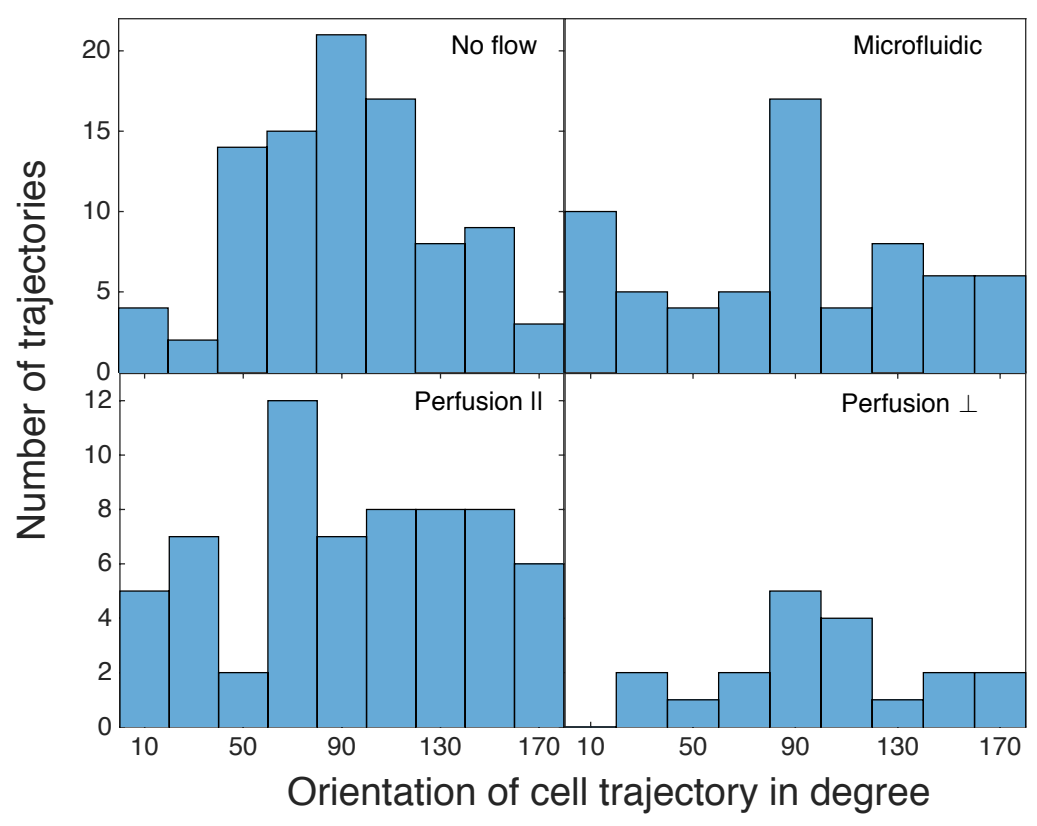

Figure 4.15: Histogram showing the orientation of $D . d$. cell trajectories on optical fibers of all four different experimental setups with fiber radius $r=80 \mu \mathrm{m}$. The orientation is defined by the direction of the convex hull compared to the axis of the optical fiber, where $0^{\circ}$ corresponds $0^{\circ}$ of the migration angles, or in other words, along the fiber axis. Besides the setup in the perfusion chamber with flow parallel to the fiber axis $\left(70^{\circ}\right)$, for all setups the highest peak is found at $90^{\circ}$.

the lengths of $\mathbf{p}_{\mathbf{1}}$ and $\mathbf{p}_{\mathbf{2}}$ as measure of the elongation of the convex hull.

$$
\text { elong }=\frac{\left\|\mathbf{p}_{1}\right\|}{\left\|\mathbf{p}_{\mathbf{2}}\right\|}
$$

For the histogram, we use the direction of the first component of convex hulls with an elongation higher than two, elong $>2$. In all categories, we can see the trend that more trajectories have directions close to $90^{\circ}$. In the histogram corresponding to $\mathrm{No}$ flow conditions, the peak at $90^{\circ}$ is surrounded by high values in the bins of $70^{\circ}, 110^{\circ}$ and $130^{\circ}$. The results of the microfluidic channel experiment show a very clear peak 


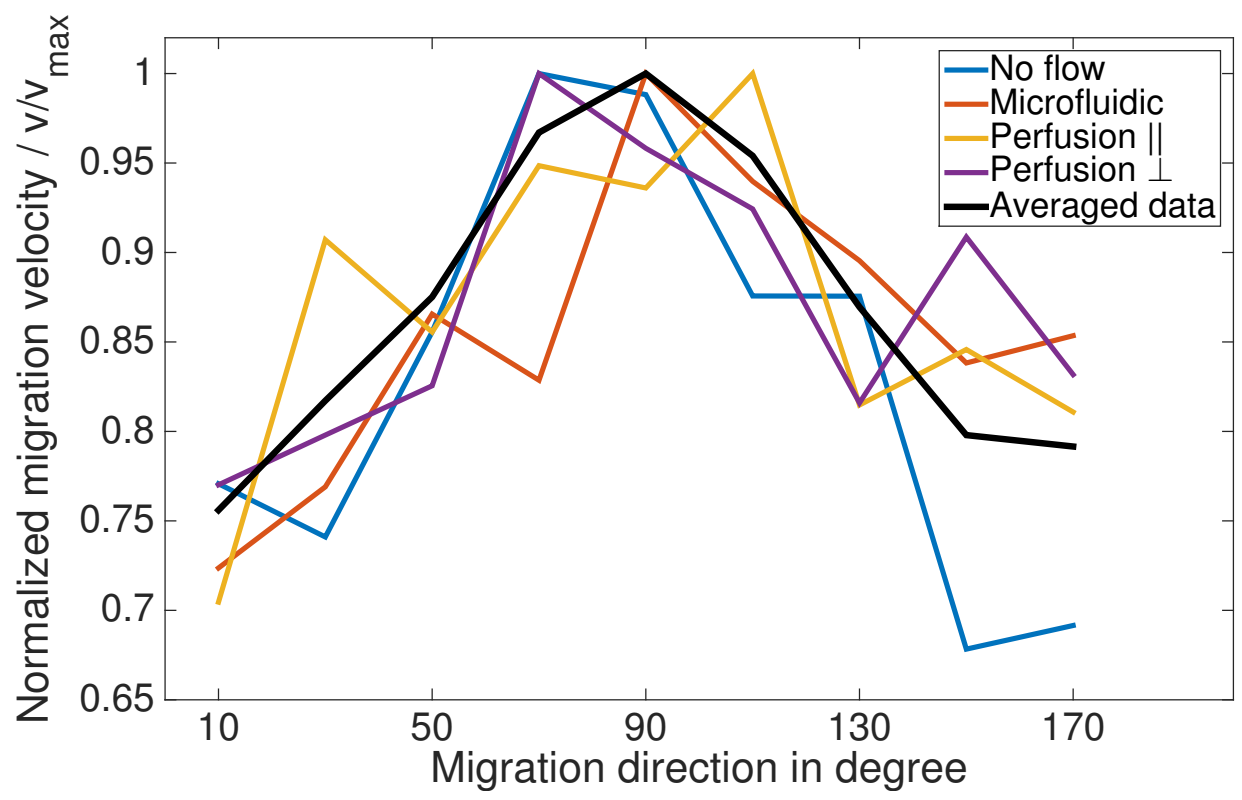

Figure 4.16: Angle dependence of cell migration velocity of all different experimental setups. The velocities have all the tendency to be higher in the curvature direction, with the maximal velocities found in the directions between $70^{\circ}$ to $110^{\circ}$.

at $90^{\circ}$. In the histogram concerning the perfusion setup with fluid flow parallel to the optical fiber we find the peak at $70^{\circ}$ and additionally high values for all bins up to $150^{\circ}$. This may be due to the experimental setup. In this setup there is still a chemotactic signaling possible for cells downstream on the fiber,as this analysis shows that several cell trajectories are shifted from $90^{\circ}$. Finally we observe that the maximum for perfusion flow experiment with the flow direction perpendicular to the fiber axis is also at $90^{\circ}$. Here the overall number of trajectories is small compared to the other data sets.

Thus, we can show the anisotropy of the cell migration, or the curvotaxis, with this measure for all experimental setups.

Besides the migration and the orientation angles of the trajectories, the migration 
velocity of the cells is also found to be maximal along the curved direction as shown in Figure 4.16. In this plot we show the velocities of cells averaged in bins of $20^{\circ}$ of their migration direction and normalized by the maximum of each curve. We plot the normalized migration velocity against the center of the bins for all different setups in different colors. Additionally we plot the average of all data in black. We observe that the curves for each category are noisy. The maxima for all curves are reached either for $70^{\circ}, 90^{\circ}$ or $110^{\circ}$ and the minima are reached at the borders. The absolute values of the migration velocities are changing with the experimental setup, why we normalize them. This change is due to the cell-to-cell variability and due to a rather small set of cells in the case of the perfusion setup with perpendicular flow. For no flow conditions and the perfusion setup with parallel flow the velocity towards the curved direction is with about $12 \frac{\mu \mathrm{m}}{\mathrm{min}}$ comparable to typical chemotactic average velocities. For the microfluidic channel setup as well as for the perfusion setup with perpendicular flow the maximal velocities are slightly below the average velocity of chemotaxis. Cells that migrate along the direction of curvature are also migrating faster than cells that migrate in any other direction. Hence we have yet another confirmation of the curvotaxis.

Now we present the average velocities $v_{\perp}, v_{\|}, v_{\text {total }}$ of the different experimental setups in Figure 4.17. The mean velocities differ between the different experimental setups. The smallest velocities are found for the experiments in the perfusion chamber with fluid flow perpendicular to the fiber axis. On the one hand this set of cells (23) is the smallest, and cell-to-cell variability can play still a role here. On the other hand in this setup, chemotaxis is excluded strictly. This indicates that the possible chemotaxis in the other setups could have still influenced the migration speed. 


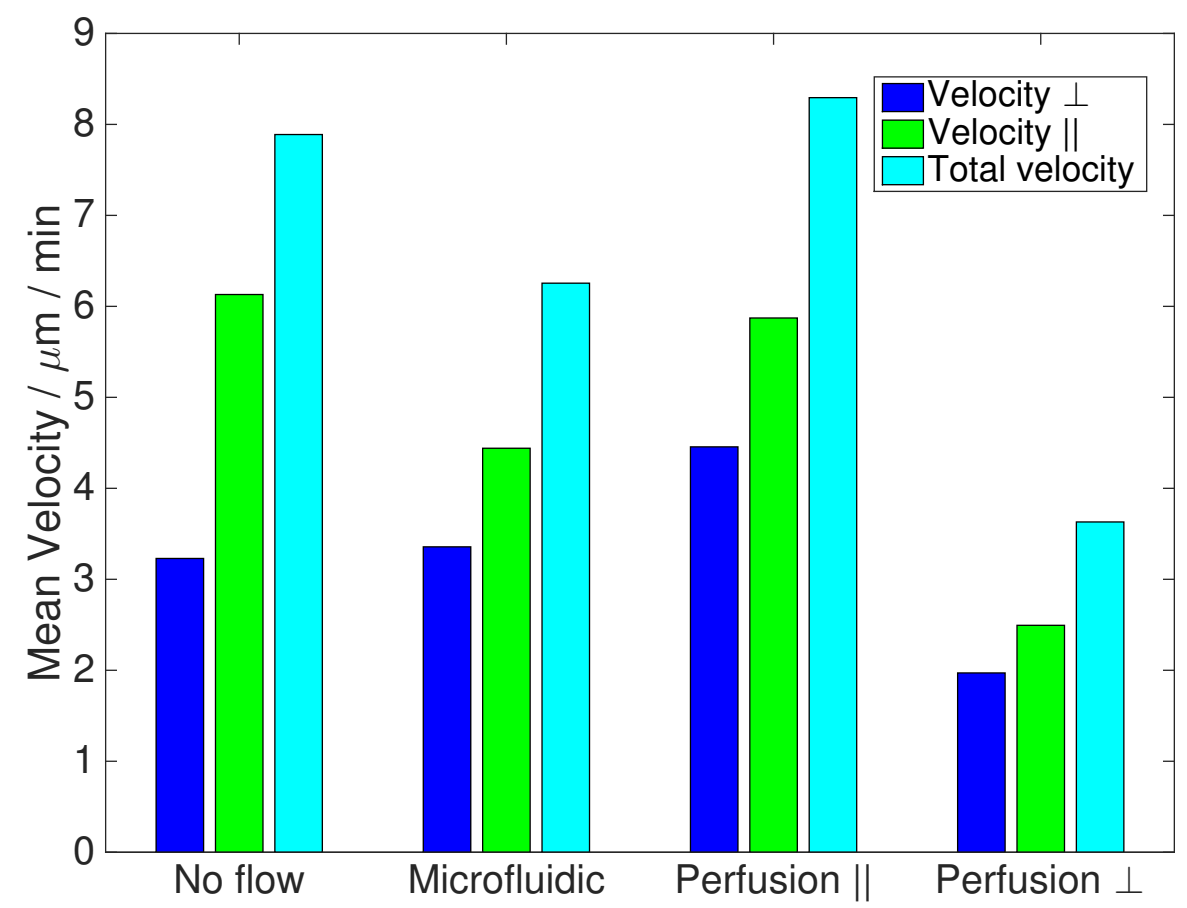

Figure 4.17: Velocities $v_{\perp}, v_{\|}$, and $v_{\text {total }}$ for all four experimental setups. The velocity in curvature direction $v_{\|}$is always higher than the velocity in fiber direction $v_{\perp}$. The velocities differ between the setups and are smallest for the perfusion setup with flow perpendicular to the fiber axis.

Additionally we plot in Figure 4.18 the Curvotactic Anisotropy Parameter $(C A P)$ corresponding to the category as defined in section 4.2.5. The $C A P$ is very useful to characterize the cell migration and its anisotropy. The values of the $C A P$ are between $C A P_{\text {noflow }}=1.90$ and $C A P_{\text {Perfusion } \perp}=1.27$. The effect of curvotaxis is visible for all setups. The comparison with a random walk (red dashed line in Figure 4.18) shows that even the smallest value of the $C A P, C A P_{P e r \perp}=1.27$, has an anisotropy in the cell migration velocity of more than $27 \%$. This clearly shows that the curvotaxis is taking place without influence of cell chemotaxis.

Hence we find for all four different experimental setups an anisotropy towards the 


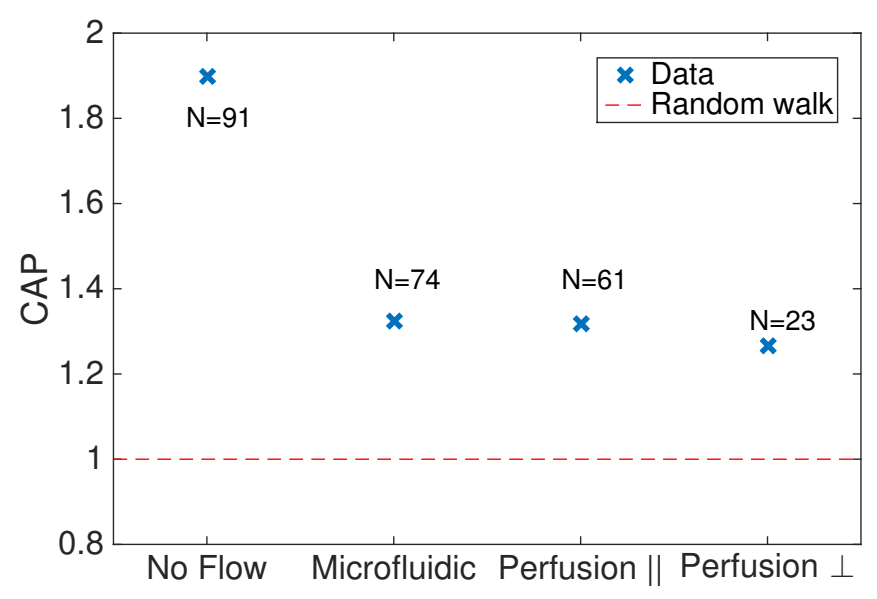

Figure 4.18: For the different experimental setups the values of $C A P$ differ. In no flow conditions the anisotropy is most pronounced. In the case of fluid flow parallel to the cylinder axis, the anisotropy is lower and consistent for both setups. In the setup with fluid flow perpendicular to the cylinder axis the anisotropy measured by $C A P$ is smallest, but still $20 \%$.

curved direction. The mechanism that directs the $D$. $d$. cells to migrate in a high curvature direction is still working if we prevent biochemical signaling via diffusing cAMP.

\subsubsection{Curvature-Dependence of Curvotaxis}

The effect of the fiber radius on the curvature induced migration of $D . d$. cells was also investigated. Therefore we used optical fibers and self-pulled glass capillaries. The investigated fiber radii range between $25 \mu \mathrm{m}$ and $80 \mu \mathrm{m}$. A reasonable way to analyze the differences is the curvotactic anisotropy parameter $C A P$. The experimental setup in this section is exclusively the perfusion chamber in which the fiber was placed perpendicular to the fluid flow as shown in Figure 3.5. The cell line used for all these experiments was ACA-null cells. 
In Figure 4.19, the $C A P$ is shown as a function of the fiber radius in $\mu \mathrm{m}$. All values for the $C A P$ are above 1 , hence they show an anisotropy in the curvature direction.

There is a trend, suggesting that the curvotactic efficiency decreases for higher fiber radii as shown by the regression that is plotted in Figure 4.19 as the blue, dash-dotted line. To gain more results for higher radii is very challenging as the imaging becomes difficult due to increasing fiber radii. The fit has the following functional form:

$$
\begin{aligned}
m_{W T, \text { Cylinders }} & =4.2 \cdot 10^{-3} \frac{1}{\mu \mathrm{m}} \pm 7.0 \cdot 10^{-3} \frac{1}{\mu \mathrm{m}} \\
c_{W T} & =1.55 \pm 0.49
\end{aligned}
$$

The errors correspond to the $95 \%$ confidence interval of the fit. The y-interception of this fit is $c_{W T}=1.55$ and still bigger than one for the maximal error. The error of the slope has a confidence interval is slightly bigger than its value.

\subsubsection{Biochemical Origins of Curvotaxis}

To determine the mechanism that leads to curvotaxis we also investigated mutant cell lines, namely Myosin-II-null, lacking contractile forces, and PTEN-null cells, which are unable to polarize. As mentioned in section 2.3.3, the two cell lines show hampered chemotaxis. Myosin-II-null cells show reduced rates of cell division and migration velocities, but they still migrate in a chemotactic gradient. In PTEN-null cells, the chemotactic signaling equilibrium between front polarization via PI3K and 


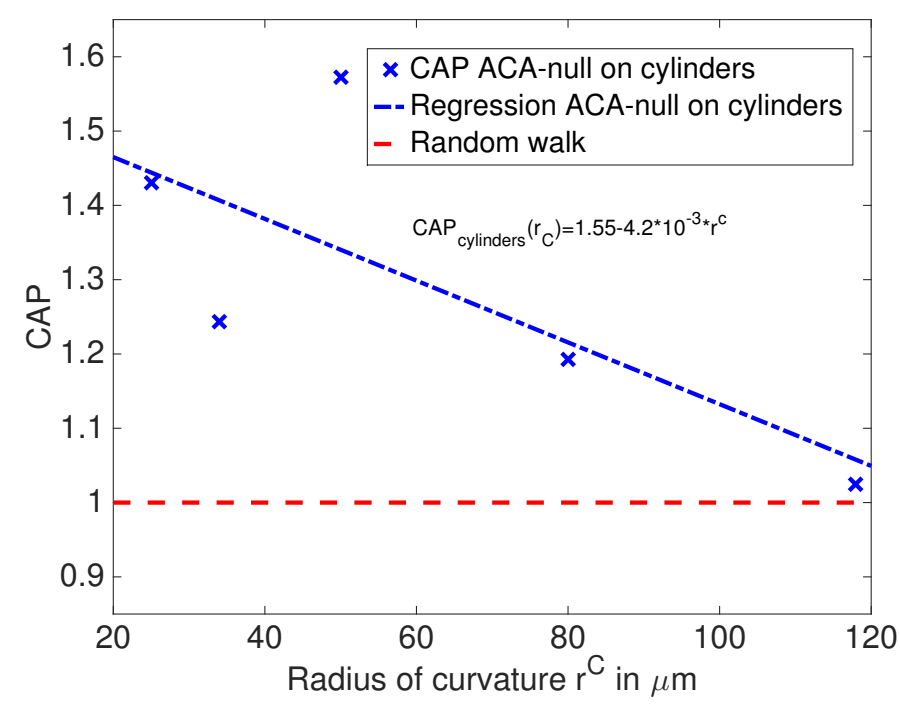

Figure 4.19: Plot of the Curvotactic Anisotropy Parameter $(C A P)$ against the fiber radii of optical fibers. The values of the curvotactic efficiency are between 1.5729 and 1.0243. There is a small trend visible that towards higher radii the $C A P$ is decreasing. The regression leads to a dependence which is also shown.

back polarization via PTEN is disturbed. Also these cells are able to migrate in a chemotactic gradient.

In our experiments we could not find any curvotactic cell migration of either of the two cell types on a fiber placed inside the perfusion chamber with fluid flow perpendicular to the fiber axis. As a further check in addition to the genetic mutations we also used chemical inhibitors for Myosin-II (blebbistatin $c=100 \mu \mathrm{M}$ ) or respectively PTEN (LY29004 $c=50 \mu \mathrm{M}$ ). In those experiments, we found the cells to migrate properly until we added the drug. After the drug was added, we could not find any migration anymore. The cells were only wriggling around but there was no net displacement, instead of performing a random motion as would be suggested on a flat surface. Hence we conclude that a contractile cytoskeleton and the abiltity to polarize are both necessary for curvotaxis. 


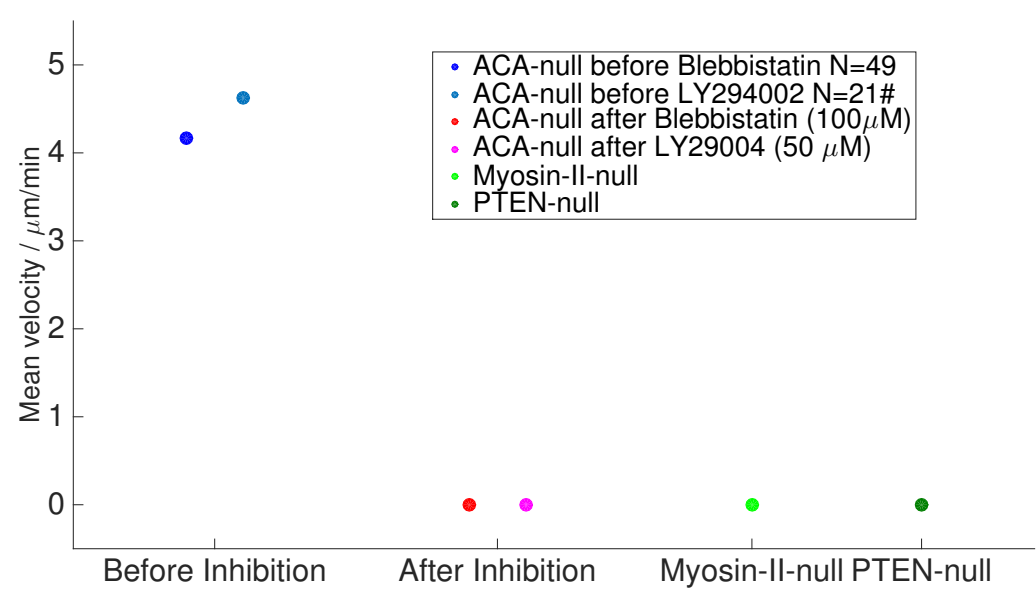

Figure 4.20: Mean migration velocity of cells before and after chemical inhibition and knockout mutants.

\subsection{Curvotaxis on Wrinkled Substrates}

To understand $D . d$. migration on curved substrates, we were also investigating the effect of negatively curved geometries. Therefore we first investigated cell migration inside of glass capillaries. These investigations indicated that the migration in negatively curved regions also appears to be anisotropic. Because of several difficulties in the experimental setup with the capillaries, like destruction due to bubbles and cell death due to a lack of oxygen, we decided to take a step towards more complex geometries and started to investigate the migration of $D . d$. cells on a sinusoidal wave-like geometry. It is produced by a wrinkling instability in a composite material made of PDMS and polystyrene as described in section 3.2.3.1. The advantage of such a substrate is that the cells are facing both positively as well as negatively curved regions. This leads to a situation for the cells that more likely reflects the natural environment. 
To compare the cell migration on this wave-like substrate, we calculate the radius of curvature for each device. The main analysis of the cell migration on the wave-like substrates is done according to the analysis of curvotaxis on the cylinders, namely the migration angle distribution, the track orientation angle distribution, the angle dependent cell velocity and the curvotactic anisotropy parameter. Additionally we include the cell orientation angle distribution and split the migration direction into positive and negative curvature depending on the position of the cell.

As above for the curvotaxis on cylinders, one examplary substrate will be shown in detail before the data presentation for different radii of curvature.

\subsubsection{Exemplary Curvotaxis Result on Wrinkles}

In this section we show an exemplary result of cells migrating on a wrinkled substrate. In this case, the wavelength of the pattern is measured optically. We find the wavelength $\lambda=71.3 \mu \mathrm{m} \pm 3.0 \mu \mathrm{m}$. The amplitude of the wrinkles have been measured in several experiments to be very consistently at approximately $A=1.5 \mu \mathrm{m}$. Hence the maximal radius of curvature reached in the extrema of the wave-like structure is $r_{\text {max }}^{c}=85.8 \mu \mathrm{m} \pm 7.4 \mu \mathrm{m}$. Preventing the chemotactic signaling of $D$. $d$. cells on a wrinkled substrate is more difficult than on the cylinders, as the dimensions of the wrinkled substrates are in the order of a few millimeters squared. Instead of having to deal with the challenge to flush away the cAMP the cells produce, we decided to use a cell line that is unable to secrete cAMP (ACA-null) - but apart from this defect - retains all functionality of wild-type $D . d$. cells. Hence we can use a very simple setup without any fluid flow for the experiments with wrinkled substrates. We simply place the wrinkled device on a glass bottom petri dish. In the following, 


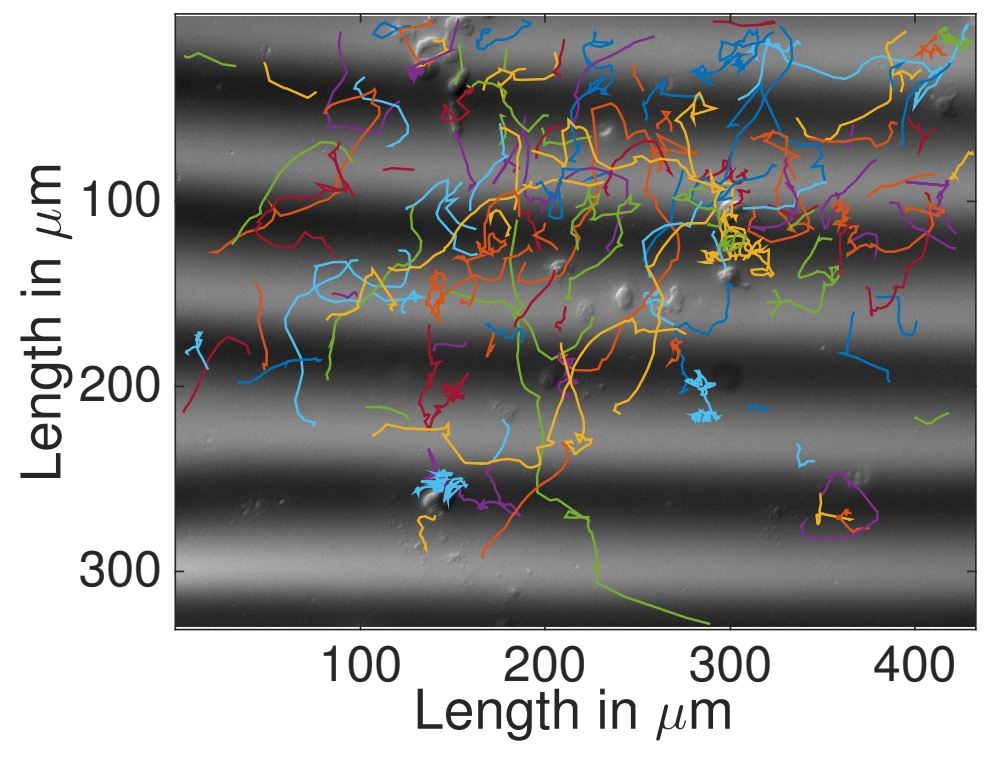

Figure 4.21: $D$. $d$. cells migrating on polystyrene wrinkles. The graphic shows an overlay of the initial DIC image of the experiment which is overlayed by all trajectories with an displacement of at least $10 \mu \mathrm{m}$ in either $\mathrm{x}$ - or y-direction. In this way we exclude non-migrating or dead cells. The geometry of the sinusoidal wave-like structure can be seen due to the differences in the brightness of the DIC image.

we show the results of the migration angles, track orientation angles, cell orientation angles and migration velocities for the wrinkled substrate with $r_{\text {max }}^{c}=85.8 \mu \mathrm{m}$.

As a starting point in Figure 4.21 we plot a DIC image of the wrinkled substrate overlayed by all trajectories found by the tracking algorithm that have a minimal displacement of $10 \mu \mathrm{m}$ that corresponds approximately to one cell length. Compared with the curvotaxis experiments on cylinders, there is no immediately obvious preferred migration direction. The statistical analysis of the migration directions is shown in Figure 4.22. The histogram of all migration angles of the specific device is 


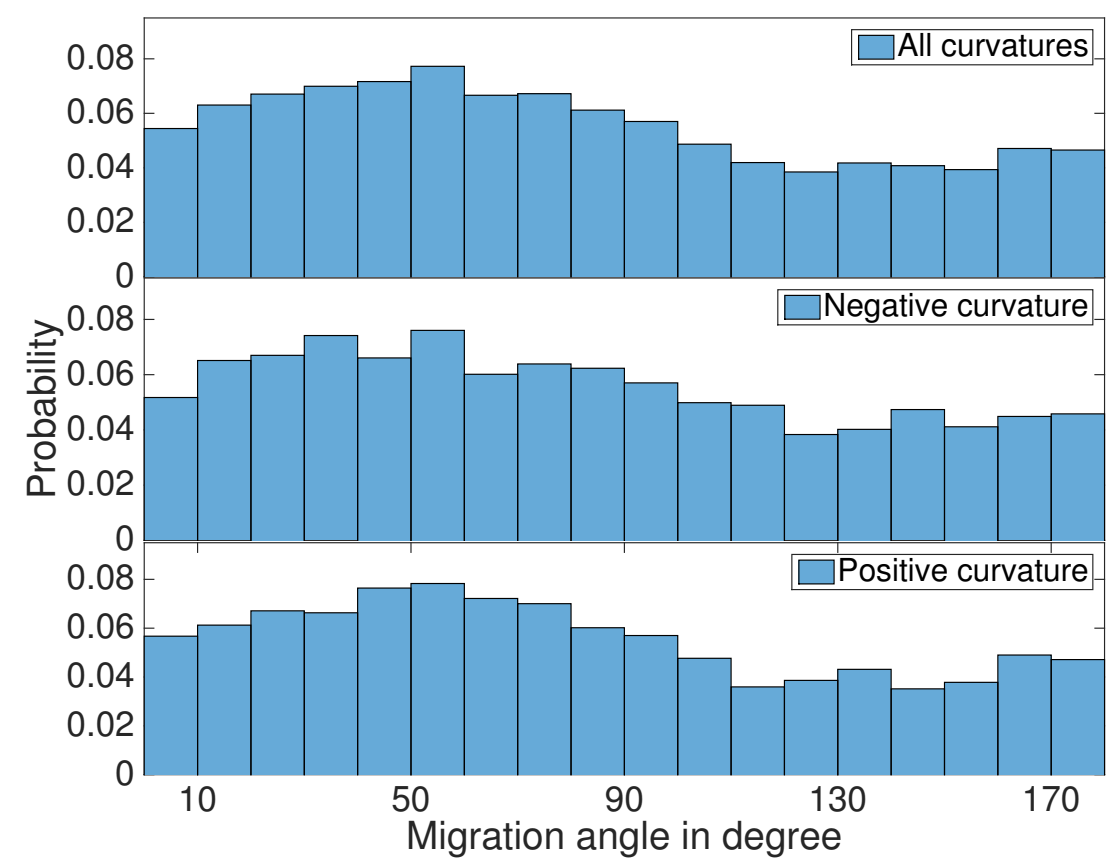

Figure 4.22: Histograms of migration angles on wrinkles with $r_{\max }^{c}=85.8 \mu \mathrm{m}$ for all cells and divivded in migrating steps for cells in negatively respectively positively curved regions. We exclude cells that do not migrate at least $10 \mu \mathrm{m}$ as well as single steps where the total velocity is zero. We find no differences in the distribution of the migration direction dependent on whether the cells are migrating in negatively or positively curved regions.

shown on the top. The peak of a wide range of higher values is in the bin between the migration direction of $50^{\circ}$ and $60^{\circ}$. In addition, we find high values between $0^{\circ}$ and $100^{\circ}$ and smaller values between $110^{\circ}$ and $180^{\circ}$. Dividing the cell migration in the negatively or positively curved areas of the substrate, this leads to the result shown in the histograms in the middle and at the bottom. Besides some minor details, the structure of these plots is very similar to each other and therefore also to the histogram at the top which is the combination of both. Hence we do not find a strong difference between positively and negatively curved regions. 


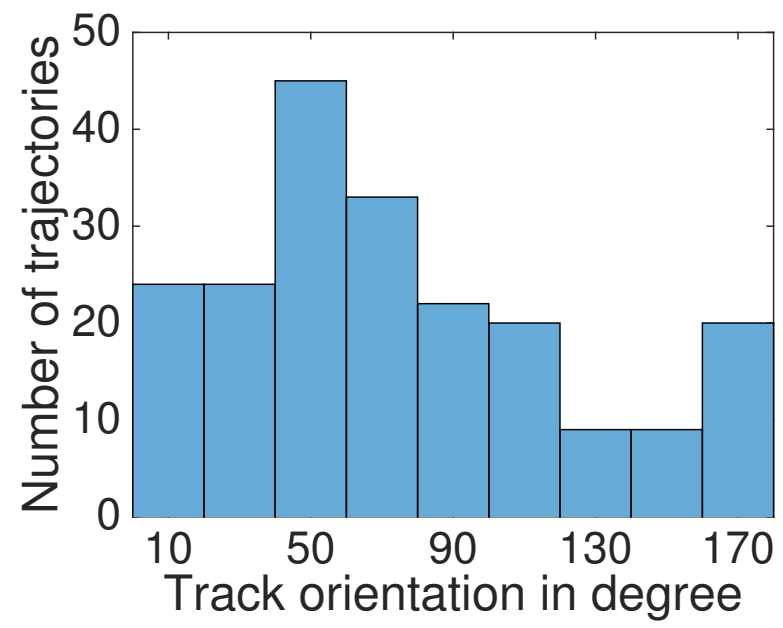

Figure 4.23: Histogram showing the orientation of $D$. $d$. cell trajectories on a wrinkled substrate with a maximal radius of curvature $r_{\text {max }}^{c}=85.8 \mu \mathrm{m}$. The orientation is defined by the direction of the convex hull compared to the axis of the optical fiber, where $0^{\circ}$ corresponds $0^{\circ}$ of the migration angles. The orientation direction of the trajectories shows a peak at $50^{\circ}$.

The second parameter to quantify the anisotropy of the $D . d$. curvotactic migration is the orientation of the cell trajectories. We access this orientation as the direction of the first principal component of the convex hull of motile and directed trajectories. We call a trajectory directed if its first principal component is at least twice as high as the second component. The definition of motility is the same as above. The histogram in Figure 4.23 shows the directions of the convex hulls. The peak of the distribution is consistently in the bin between $40^{\circ}$ and $60^{\circ}$ to the peak of migration angle distribution. Hence the anisotropy of the track orientation is similar to the anisotropy of cell migration at the single step level.

The use of the automated tracking provides a more precise and objective way to determine the cell migration. Additionally, the chosen algorithm finds and tracks the cells automatically, not only the cell positions but also the cell shapes are known. The 


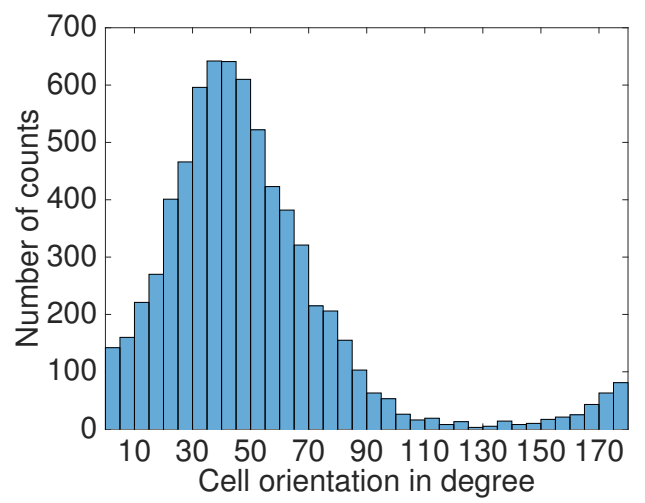

(a) Histogram of the cell orientation angle (b) of $85.8 \mu \mathrm{m}$ radius of curvature wrinkles. The tracked cells are mostly oriented in the direction of $40^{\circ}-50^{\circ}$. The very low values between $110^{\circ}-150^{\circ}$ is an image processing artefact due to deformation of cells.

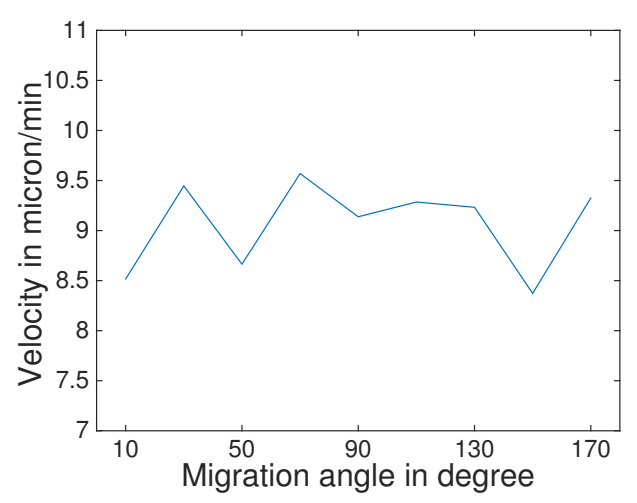

(b) This plot shows the migration velocity of $D$. $d$. cells dependent on the migration direction of the cells for the cells migrating on wrinkles with $85.8 \mu \mathrm{m}$ radius of curvature. The velocity shows no peak for any migration direction.

Figure 4.24: Cell orientation and direction dependence of velocity

manual extraction of cell shapes from the experiments on cylinders is not feasible as it would increase the time to analyze the data many-fold. We use the cell shapes in each timestep to analyze the cell orientation in this step. As polarized cells usually migrate in the direction in which they are polarized, thus we gain another measure for the anisotropy of the cell migration. The results of the experiments with a maximal radius of curvature of $r_{\text {max }}^{c}=85.8 \mu \mathrm{m}$ are shown in Figure 4.24(a).

Investigating the velocity and the $C A P$ of this experiment, we find that the velocity in along the curved direction is higher than the velocity along the flat direction. In this case the $C A P$ shows just a 5\% anisotropy, meaning it is much smaller than for the cylinder experiments, but still shows the anisotropic migration. 


$$
\begin{aligned}
<v_{\perp, 85.8}> & =4.7 \frac{\mu \mathrm{m}}{\mathrm{s}} \\
<v_{\|, 85.8}> & =4.9 \frac{\mu \mathrm{m}}{\mathrm{s}} \\
<v_{85.8}> & =7.6 \frac{\mu \mathrm{m}}{\mathrm{s}} \\
\Rightarrow C A P_{85.8} & =1.05
\end{aligned}
$$

\subsubsection{Dependence of Curvotaxis on Maximal Radius of Curvature of Wrinkles}

To investigate the influence of the geometric properties of the wrinkled substrates, we used substrates with different maximal radii of curvature. In the following Figures 4.254 .29 , we show the same properties as in the section above for four different radii of wrinkled substrates.

The migration angle histogram in Figure 4.25 shows the histogram of directions of motile cells for every step. The maxima of the cell migration histogram are consistently peaked between $40^{\circ}$ and $60^{\circ}$ for three different radii. Only for the case of $r_{\text {max }}^{C}=137 \mu \mathrm{m}$ we find no maximum in this region, instead a small peak at $90^{\circ}$.

The second parameter to quantify the anisotropy of cell migration is the orientation of cell trajectories (Figure 4.26). The results of these histograms are very consistent for the first three cases with the directions of the cell migration histograms. In those cases the peaks of the histograms are at the bin with center of $50 \mu \mathrm{m}$. In the case for $r_{\text {max }}^{C}=137 \mu \mathrm{m}$ we find a peak at $70^{\circ}$.

As further confirmation of the migration anisotropy we plot the cell orientation 


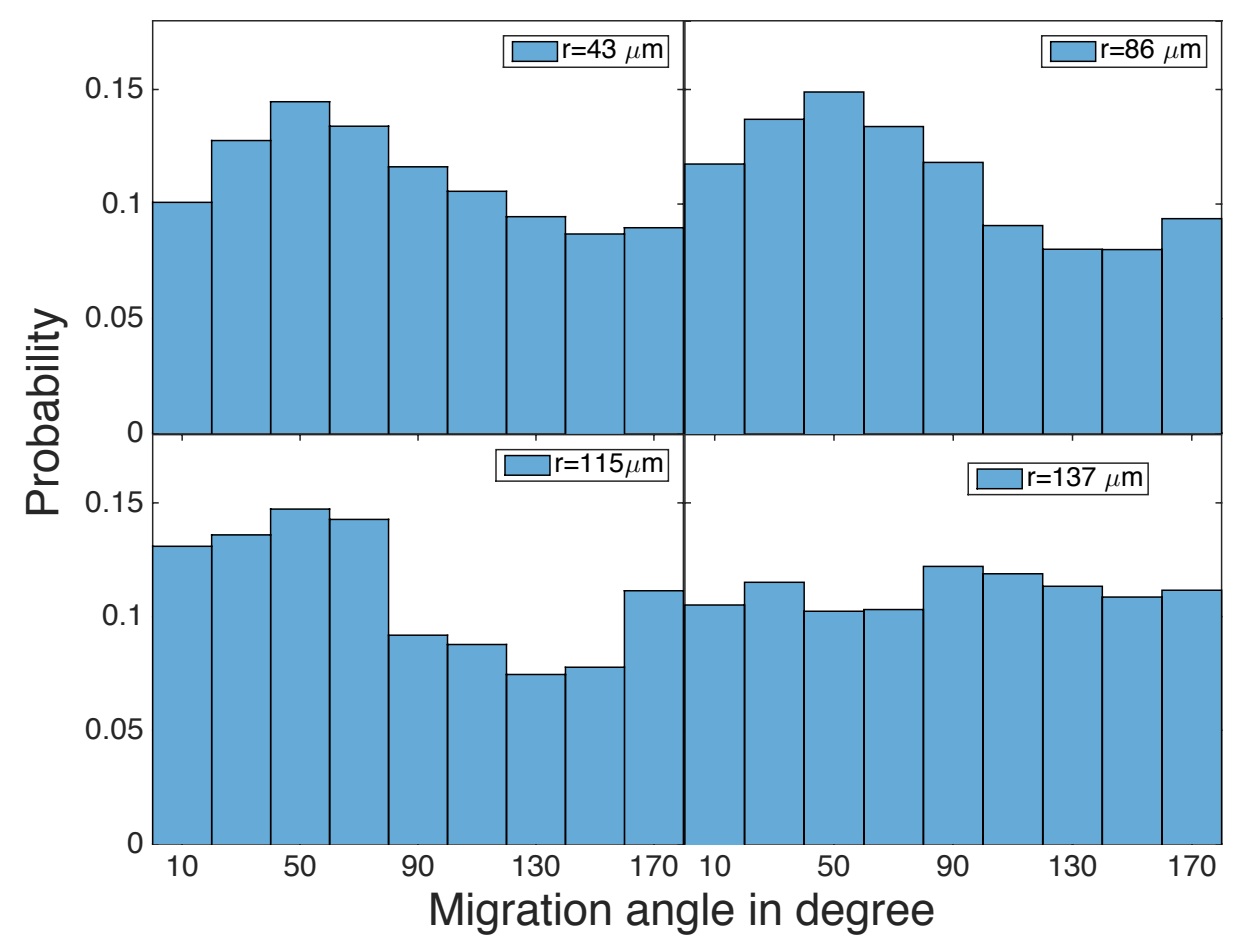

Figure 4.25: Histogram of migration angle for motile cells for all different wrinkled radii. In the first three cases $r_{\text {max }}^{C}=\{43 \mu \mathrm{m}, 85.8 \mu \mathrm{m}, 115 \mu \mathrm{m}\}$ the maxima are at the bin at $50^{\circ}$. For the biggest radius $r_{\text {max }}^{C}=137 \mu \mathrm{m}$ we find only a very small maximum at $90^{\circ}$.

with different radii of curvature. In all cases the maxima of the histogram lies between $30 \chi$ and $60^{\circ}$. The values of the maxima are also consistent with the maximal values of the track orientation histograms in Figure 4.26 .

The angle dependence of the migration velocity is shown in Figure 4.28, There is no trend visible for either of the radii. To quantify the anisotropy of the cell migration on the wrinkled substrates, we again use the curvotactic anisotropy parameter $(C A P)$. The small trend can be quantified by calculating the regression line. This line shows 


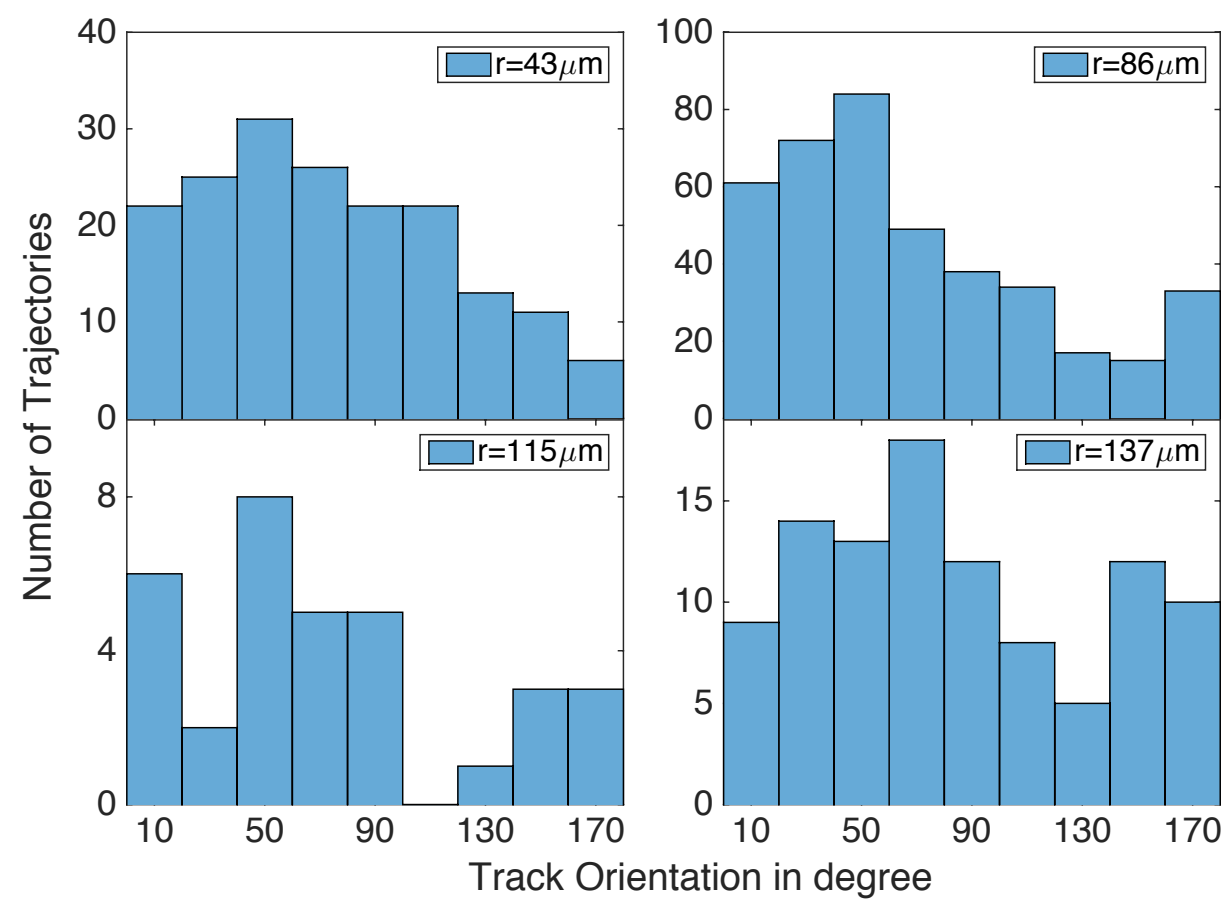

Figure 4.26: Histogram showing the orientation of $D$. $d$. cell trajectories on all wrinkled substrates. The orientation is defined by the direction of the convex hull compared to the axis of the optical fiber, where $0^{\circ}$ corresponds 0degree of the migration angles. The orientation direction of the trajectories shows peaks at $50^{\circ}$ for the three smallest radii, and $70^{\circ}$ for the biggest radius.

a decline. Its functional dependence is:

$$
C A P_{\text {wrinkles }}\left(r^{c}\right)=\left(9.5 \cdot 10^{-4} \frac{1}{\mu \mathrm{m}} \pm 1.7 \cdot 10^{-3} \frac{1}{\mu \mathrm{m}}\right) \cdot r^{C}+1.16 \pm 0.18
$$

Hence for bigger radii of curvature, the anisotropy decreases. For the biggest maximal radius of curvature $r_{\text {max }}^{C}=137 \mu \mathrm{m}$ the anisotropy is only $2 \%$. The errors correspond to the $95 \%$ confidence interval of the fit. 


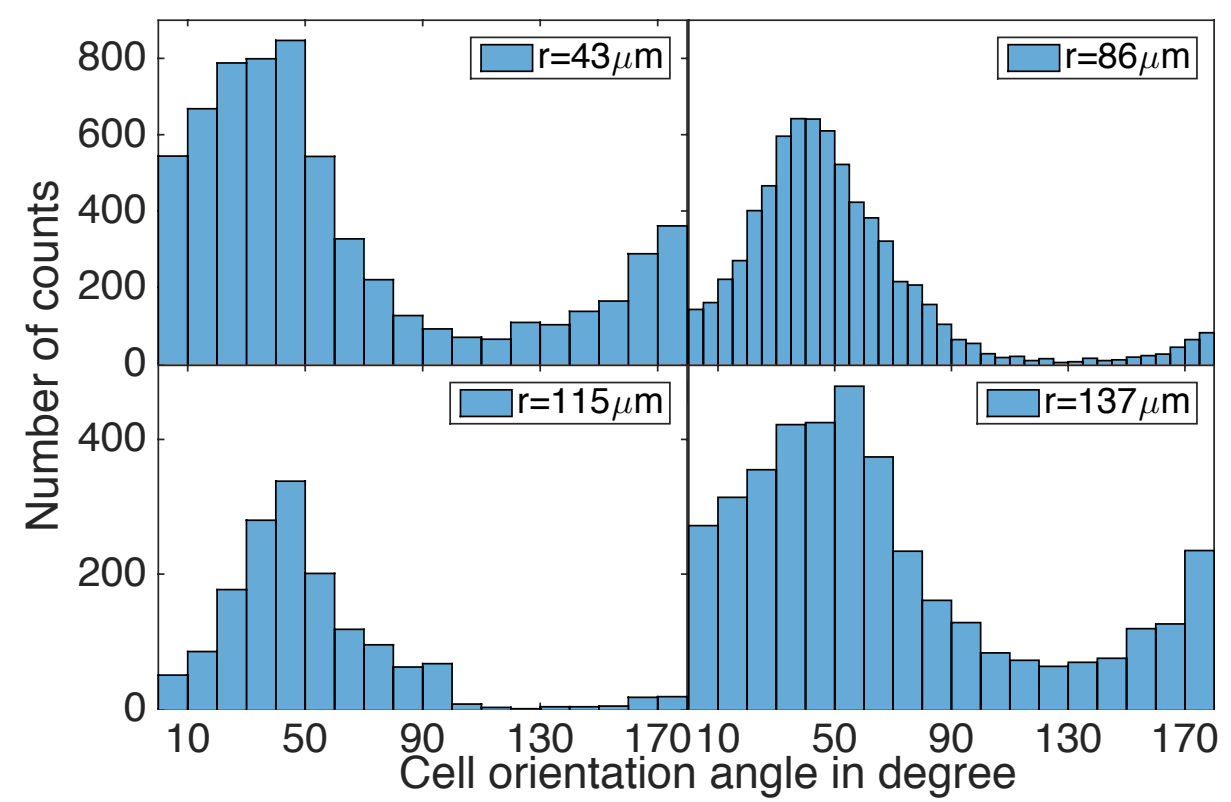

Figure 4.27: Histogram of the cell orientation angle of all different wrinkled substrate. The tracked cells are mostly oriented in the direction of $40^{\circ}$ and $60^{\circ}$. The very low values between $110^{\circ}$ and $150^{\circ}$ are an artefact of the image processing.

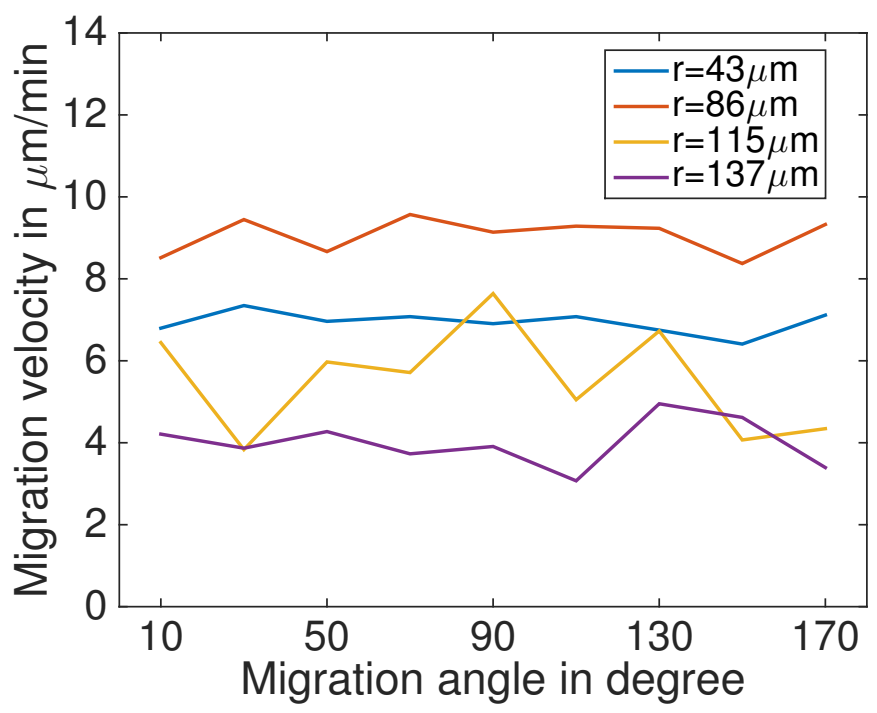

Figure 4.28: This plot shows the migration velocity of $D$. $d$. cells dependent on the migration direction of the cells for the cells migrating on all wrinkles substrates. The velocity shows no directional dependence. 


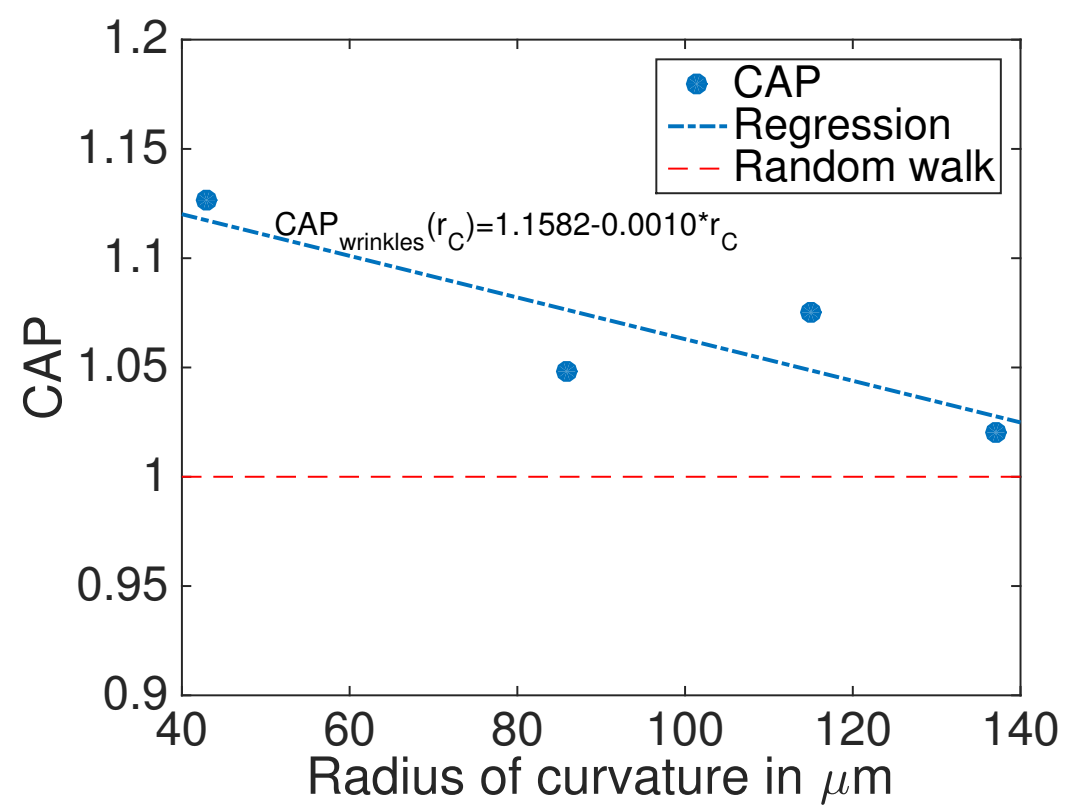

Figure 4.29: Plot of the Curvotactic Anisotropy Parameter $(C A P)$ against the radii of maximal curvature of the wrinkled substrates. The values of the curvotactic efficiency are between 1.12 and 1.02. There is a small trend visible that towards higher radii the $C A P$ is decreasing. The dependence of the fit is $C A P_{\text {wrinkles }}\left(r^{c}\right)=\left(9.5 \cdot 10^{-4} \frac{1}{\mu \mathrm{m}} \pm 1.7 \cdot 10^{-3} \frac{1}{\mu \mathrm{m}}\right) \cdot r^{C}+1.16 \pm 0.18$.

\subsection{Discussion: Cylinders vs. Wrinkles}

Finally we compare the curvotactic cell migration of the two main different experimental setups and discuss the results. Generally speaking, the main difference of the systems is that the curvature changes for the wrinkled substrates, while the curvature is constant on the cylinders. In the case of the curvotaxis on a cylinder, we find a clear anisotropy in all measures. The migration angle, the cell trajectory orientation and cell migration velocity all show maxima at $90^{\circ}$, respectively parallel to the curvature. Hence for the cell migration on cylinders we have several proofs that confirm the ansisotropic cell migration, hence curvotaxis. 


\section{Shapes (with threshold 3)}

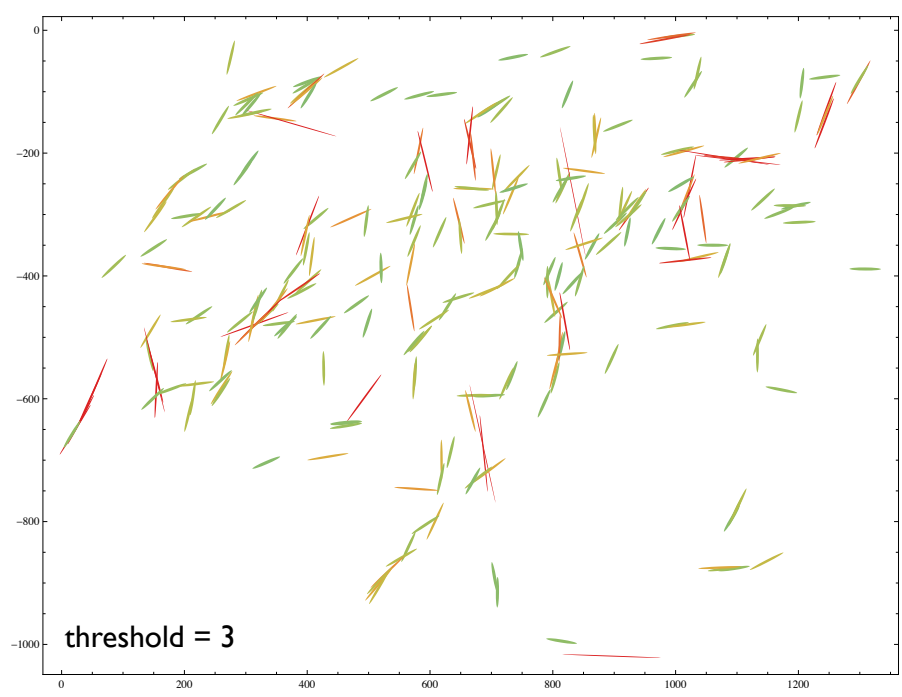

Statistics
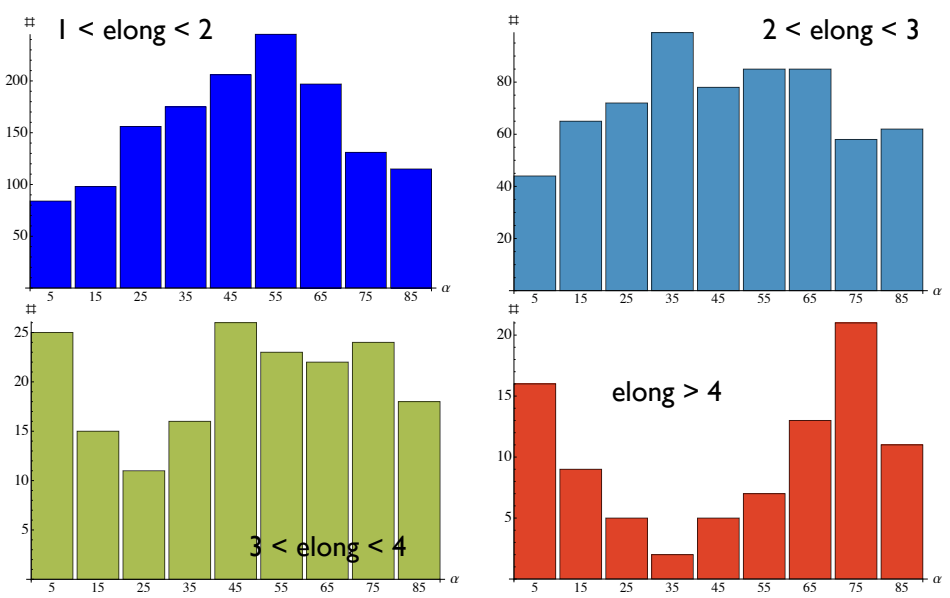

Figure 4.30: The left hand graph shows orientation and elongation of cells migrating on a wrinkled substrate. For each cell that has a elongation of at least 3 the center of mass position and an ellipse is plotted. The color code of the ellipse corresponds to its elongation (green: small elongation, red strong elongation) and the orientation of the ellipse corresponds to the orientation of the cell. The right hand graph shows histograms of the cell orientation binned for the cell elongation. For higher elongation the probability for the cells to migrate in the direction of maximal curvature and maximal curvature gradient increases. Graphs with permission of Marco Rivetti. 
Regarding the wrinkle curvotaxis, the results are different. The cell velocity does not show an angle dependence on the wrinkled substrates. The other measures are consistent and show an anisotropy in direction of about $50^{\circ}$. This result is surprising as we would then also assume that there is a peak around $135^{\circ}$ due to symmetry. A possible explanation for this broken symmetry could be the ice-skating like migration already discussed in section 2.2.2.1.

The mechanism with which the cell chooses the migrational direction is presumably actively controlled by the cell and not just a pure consequence of the surface geometry. This was proved earlier in this thesis in section 4.3.4, where without a properly working cytoskeleton no curvotactic migration was found. The cytoskeleton was manipulated in three ways, namely a knockout of the heavy chain of myosin-II, by disabling the functionality of the myosin-II head group via chemical inhibition with blebbistatin, and by dissembling actin filaments using the drug Latrunculin A. The signaling pathway known from chemotaxis is also important, as at least the polarization controlled by PI3K and PTEN were shown to be necessary for curvotactic migration. As PI3K and PTEN are responsible for the symmetry breaking of the cell, we recently started to analyze how the cell polarization affects its directionality. In the left hand graph of Figure 4.30 the positions, directions and elongations of cells from the experiment shown in section 4.4.1 are shown, starting at a threshold of 3. Here the elongation is shown in the geometry of each ellipse as well as in its color (green: small elongation, red: strong elongation). In the histograms on the right hand side of Figure 4.30, the directionality of the cells is shown in four different bins for the elongation of the cells. For the two smallest elongation bins the maxima of the cell orientation directions are at $55^{\circ}$ respectively at $35^{\circ}$, which is consistent with Figure 4.24(a). The higher the elongation is, the more pronounced the value at very 
high and very small orientation directions gets. In the case of cell elongation larger than 4 , we can observe a biphasic behavior with one peak at $5^{\circ}$ and a second and higher peak at $75^{\circ}$. That suggests that more elongated cells are more likely to choose the direction with highest curvature and highest curvature gradient.

Apart from these findings, we additionally found a new cell behavior that we call probing. A cell was still adherent at the cell rear, but with the cell front it was probing the substrate in various directions before it chose the its new migration direction.

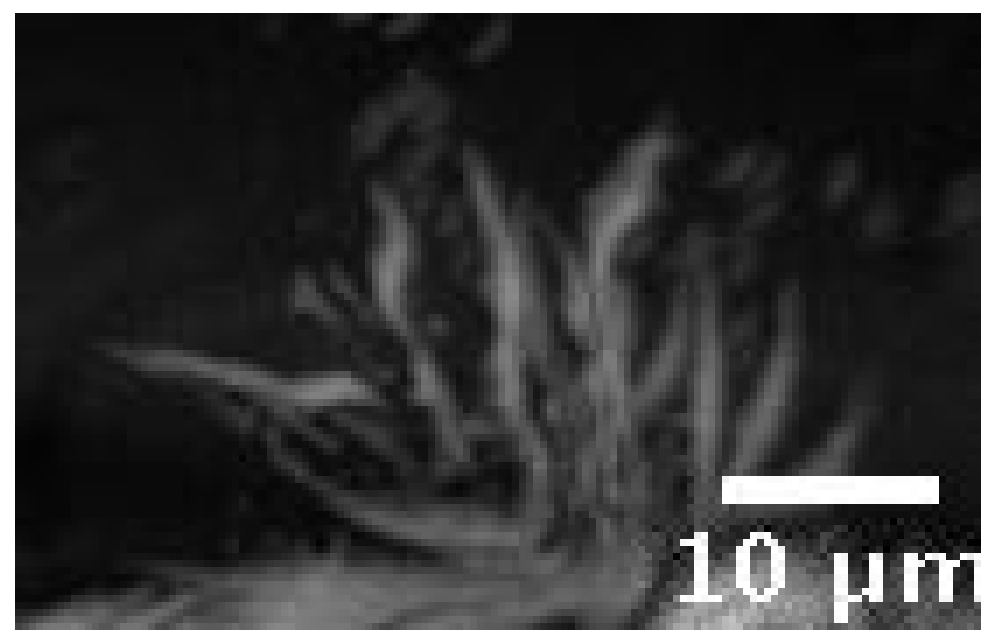

Figure 4.31: $D$. $d$. cell probing the direction to take on wrinkled substrate. Due to the use of substrating image technique we can visualise the positions the cell took for several timesteps in bright colour, while the backround is black. Hence we can see that the basal part of the cell was staying fixed while the front part was probing several direction, before it chose a direction to move further.

This can be seen in Figure 4.31 on a wrinkled substrate. The positions of the cell trajectory are shown in such a way that all the area the cell has explored is highlighted in white, while the substrate is dark. This was achieved by subtracting the average image from the image series and afterwards plotting the maximum intensity. This behavior was also seen in the experiments on the cylinders, but less frequent than in 
the experiments on the wrinkled substrates. This may be due to the more complex geometry and the changing curvature which the cell is exposed to. A cell may be exposed to positive and negative curvature when being close to the turning point of the wave-like structure. It would be reasonable for the cell to prevent this to happen by choosing the direction in this probing manner. Also the preferred migration direction could be due to the fact that the change of the curvature the cell is exposed to, is less steep than for migration exactly parallel to the curved direction.

As final result, we show the results of the cylinder data for ACA-null cells together with the results of the experiments on the sinusoidal substrate in Figure 4.32. As already mentioned in section 4.4.1, ACA-null cells need to be used for the wrinkled substrates as they lack the ability to produce cAMP.

If we compare the two different setups with each other we realize that the slope of the fitted line for the experiments on wrinkles $m_{\text {Wrinkles }}=9.5 \cdot 10^{-4} \frac{1}{\mu \mathrm{m}} \pm 1.7 \cdot 10^{-3} \frac{1}{\mu \mathrm{m}}$ is only around one quarter of the value for the cylinder experiments $m_{\text {Cylinders }}=4.2$. $10^{-3} \frac{1}{\mu \mathrm{m}} \pm 7.0 \cdot 10^{-3} \frac{1}{\mu \mathrm{m}}$. The intercept of the fitted line with the $\mathrm{y}$-axis $c_{A C A \text {, wrinkles }}=$ $1.16 \pm 0.18$ is also very small compared to the results of the cylinder data. It suggests a maximal anisotropy of around $16 \%$ compared to $50 \%$ in the case of cylinder curvotaxis $c_{C y l i n d e r s}=1.55 \pm 0.49$. We find on both experimental setups a curvature dependent $C A P$, the influence of the curvature and the value of the $C A P$ is higher on the cylinders than on the sinusoidal substrates. The importance of the actomyosin cortex as well as the importance of cell polarization was shown due to the mutant experiments with (Myosin-II-null and PTEN-null) on the cylinders. 


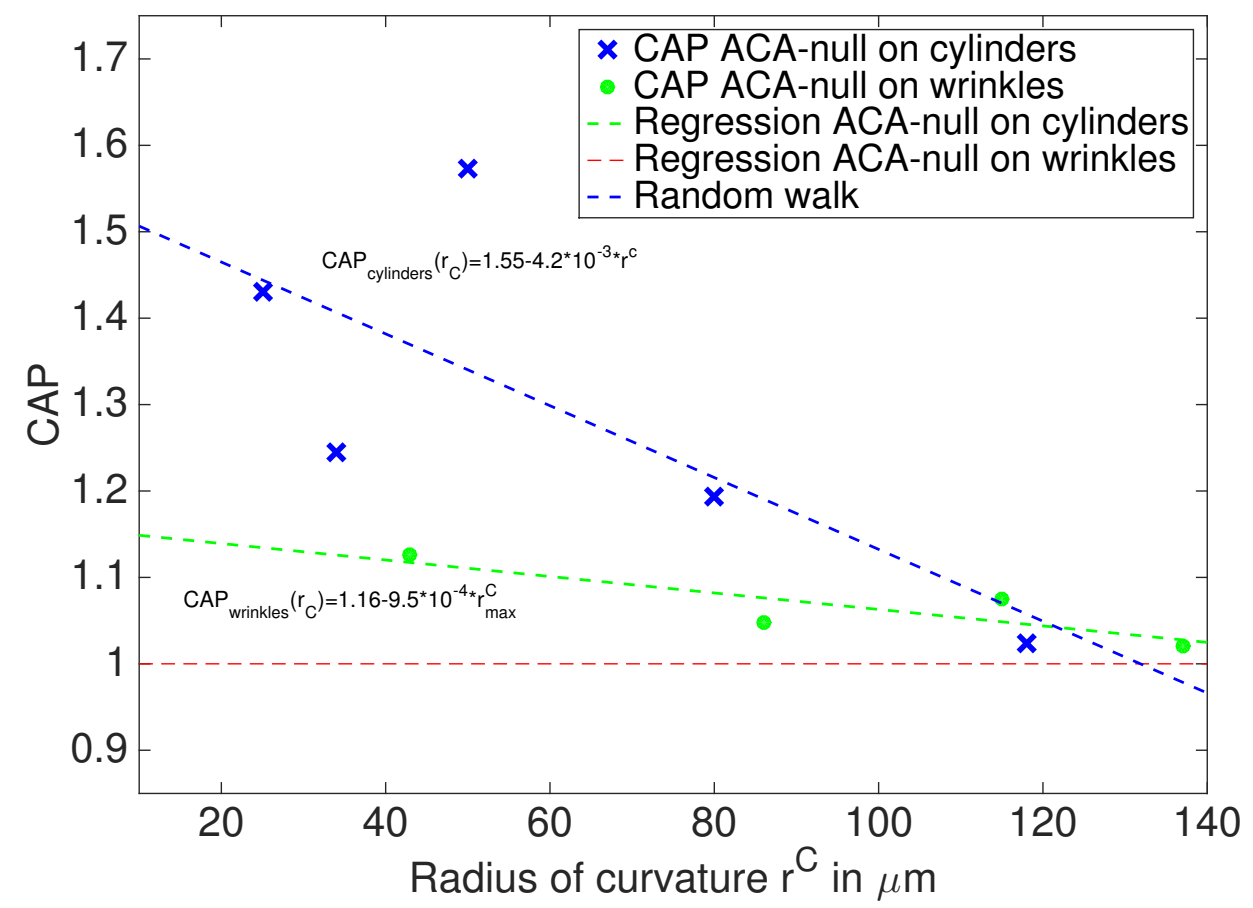

Figure 4.32: Plot of the Curvotactic Anisotropy Parameter $(C A P)$ against the radii of maximal curvature of the wrinkled substrates or fiber radius. The blue crosses show the ACA-null cells on cylinder data, while the green circles denote for the ACA-null data on wrinkled substrates. The values of the $C A P$ are between 1.5729 and 1.0205. There is a trend visible that towards higher radii the $C A P$ is decreasing. The regression leads to a dependence which is also shown. The slope of the regression lines for the cylinder data is $\left(m_{A C A-n u l l, C y l i n d e r s}=\right.$ $4.2 \cdot 10^{-3} \frac{1}{\mu \mathrm{m}} \pm 7.0 \cdot 10^{-3} \frac{1}{\mu \mathrm{m}}$, while the slope from the wrinkled substrate data is just one third of it $m_{A C A-n u l l, w r i n k l e s}=9.5 \cdot 10^{-4} \frac{1}{\mu \mathrm{m}} \pm 1.7 \cdot 10^{-3} \frac{1}{\mu \mathrm{m}}$. The interception with the $\mathrm{y}$-axis is different for all cases $c_{A C A-n u l l, C y l i n d e r s} 1.55 \pm$ $0.49, c_{A C A-\text { null,wrinkles }}=1.158 \pm 0.18$. The errors are corresponding to the $95 \%$ confidence intervals of the fits. 


\section{CHAPTER 5 \\ LPattern Formation in the Actin Cortex}

The second research chapter of the thesis is concerned with the signaling pathway of $D . d$. cells and its interplay with the cell geometry. We investigate the curvature of the cell membrane. The cross-correlation of cell geometry and the activity of the important Ras-G protein will be a central part of the study. We are seeking to find out, if cell geometry is a consequence of actin polymerization caused by the chemotactic signaling cascade of the cell or if geometry itself forces the cells to recruit the actin polymerization at the leading edge.

In fluorescent micrographs of $D . d$. cells labeled with Raf1-RBD-GFP (label for Ras-G in its activated form (Ras-G-GTP), sometimes we will refer with Ras to the Raf1-RBD-GFP fluorescence or Ras-G, as it is the Ras protein we focus on, see subsection 2.3.3) and LimE-mRFP (label for filamentous actin, see subsection 2.3.3), we can image the biochemical signals of the signaling cascade. These fluorescent micrographs can be used to extract the cell contour and, hence, the curvature of the cell membrane and the cell cortex. In this chapter, curvature always means 
two-dimensional line curvature of the cell perimeter in a micrograph, as explained in section 4.2.1. This curvature is a useful and reliable measure for the existence, formation and splitting of pseudopods. A more detailed analysis should include the three-dimensional curvatures of the pseudopod. The imaging technique for three dimensions suffers from a poor temporal resolution, as the dynamic changes of the pseudopodium are faster than the scanning rate for conventional confocal laser scanning microscopy (CLSM), which made imaging of a pseudopodium in three dimension impossible so far. A possible way to increase the temporal resolution is to use a Spinning Disc Confocal Laser Scanning Microscope (sdCLSM).

In this chapter, we will start with shortly presenting the literature of research related to this project to present its different aspects. An introduction about the used analysis tools will be given in section 5.2. Afterwards, the results for the Confocal Laser Scanning Microscope are shown, followed by the results gathered with the very high temporal resolution data of the Spinning Disc Confocal Laser Scanning Microscope (sdCLSM) gathered in cooperation with the group of Prof. Enderlein at University of Göttingen.

\subsection{Related research}

The Ras superfamily is focus of very intense research in mammalian cells and $D . d$, especially after development of an indirect marker for activated forms via Raf1-RBD (7). In the case of $D . d$. the interactions of activated Ras-G with filamentous actin was investigated and a positive feedback loop of Ras, PI3K and actin was already discussed in 2004 by the group of Firtel (67). Further studies by several groups (Devreotes, van Haastert/Kortholt and Firtel) proved the connection of the cAMP 
receptor cAR1, Ras, PI3K, PIP 3 and actin at the leading edge of a cell (39, 43, 47, 91).

Recent literature is strongly highlighting the crucial importance of Ras activation for the process of chemotaxis, as well for cAMP chemotaxis as for folate chemotaxis (17). The activation of Ras has become a marker for symmetry breaking and hence directional sensing and chemotaxis of the chemotactic $D$. $d$. cells $(48,49)$.

Ras plays also a very important part in adaptation and memory of $D . d$. in cAMP gradients. The process of adaptation that is important in chemoattractant gradients with high mean value as well as in the so called back-of-the-wave problem. The backof-the-wave problem is the effect that $D . d$. cells only migrate in one direction when a cAMP wave is moving over them. Even though they are facing the same gradient twice, once for the arriving wave and once for the departing wave, the cells only react once, to the arriving wave gradient. This problem is under strong investigation and the hypothesis is that Ras-G plays a crucial part in the incoherent feedforward loop that controls this process of adaption (75). This hypothesis is strengthened by work of Skoge et al. (69), where both experimental as theoretical work is consistent with this hypothesis. For the experimental investigation, the Rappel group used a microfluidic wave generator that allows to sweep focussed pulses of cAMP of about $700 \mathrm{nM}$ through the channel. The theoretical model of adaption they propose contains an incoherent feedforward loop that regulates RasGTP similar as described above (75).

The alternating splitting process is known to be mediated by PLA2 (12). The different migration modules of splitting pseudopods and de-novo pseudopods are also mediated by Ras. Hence the most logical line of research is to use the techniques shown in this thesis to investigate de-novo pseudopods and the differences between de-novo and splitting pseudopods. 
In addition the Rappel group and the Devreotes group have performed simulations where the influence of Ras to the chemotactic cell migration has been investigated (35, 90). Especially Hecht et al. (35) investigated as well theoretically as experimentally the interplay of Ras and pseudopod formation, but even though they could find high correlations of Ras in pseudopodia, they were not able to make any statements about the spatiotemporal distribution and cause-and-effect relations. Contrarily I could find with the results presented in this thesis temporal anisotropies between the signals of activated Ras-G and filamentous actin. I found that the LimE is lagging behind the activated Ras-G and could first answer to this questions.

\subsection{Analysis Methods}

To analyze the above mentioned experiments, we need ways to quantify the results. The specific details of the methods to quantify the results will be discussed in this section. At first, we will show the main method to extract the data, namely the cell geometry, from micrographs. In the second part of this section, we will describe the method to correlate the different signals to learn more about their dependencies.

\subsubsection{Active Contour Cell Outline Detection}

As measure of the cell geometry, we use the cell membrane curvature from quasi-2D slices of the cell measured with CLSM (approximately $0.5 \mu \mathrm{m}$ high, usually just above the glass slide ). To calculate the curvature of the cell membrane, we need a way to find the cell circumference. This non-trivial task can be realized using the micrographs that are grey-scale images with a specific amount of pixels. The pixel 
size is dependent on the parameters of the setup, e.g. the microscope objective. In our experiments, the pixel size was between $133 \mathrm{~nm}$ and $400 \mathrm{~nm}$. Hence to find the cell membrane, respectively the cell contour, we need a more elaborate method than simple thresholding. We decided to use the active contour algorithm from Debreuve. It uses a discrete variational approach to determine the cell outline via the shape gradient and is presented in (22) in greater detail.

This variational approach can be formulated as a minimization problem of an effective energy, dependent on properties of the cell. This energy may be written as a sum of a domain integral and a boundary integral as

$$
E(\Gamma)=\int_{\Omega} \phi_{f}(\Gamma, x) \mathrm{d} x+\int_{\Gamma} \varphi_{f}(s) \mathrm{d} s
$$

In this equation, $\Omega$ corresponds to an area that is in mathematical terms an open set in $\mathbb{R}^{2}$. The oriented boundary of that area is characterized by $\Gamma$ and $s$ is its arc-length parameterization. The key feature of the energy is its property of having a unique global minimum which is denoted with $\Omega^{*}$, corresponding to the cell domain.

The minimization of this energy is possible to achieve by starting with an initial contour and deforming it iteratively in a way that causes the energy to decrease in every step. This process is known as active contour method. To find the actual boundary of the cell this algorithm uses the shape gradient of the image.

\subsubsection{Kymographs}

After the position of the cell circumference is found, we can use this position to extract the cell curvature directly from this data. Additionally, we can use the cell circumference to find the fluorescence intensity of the investigated labels in the 


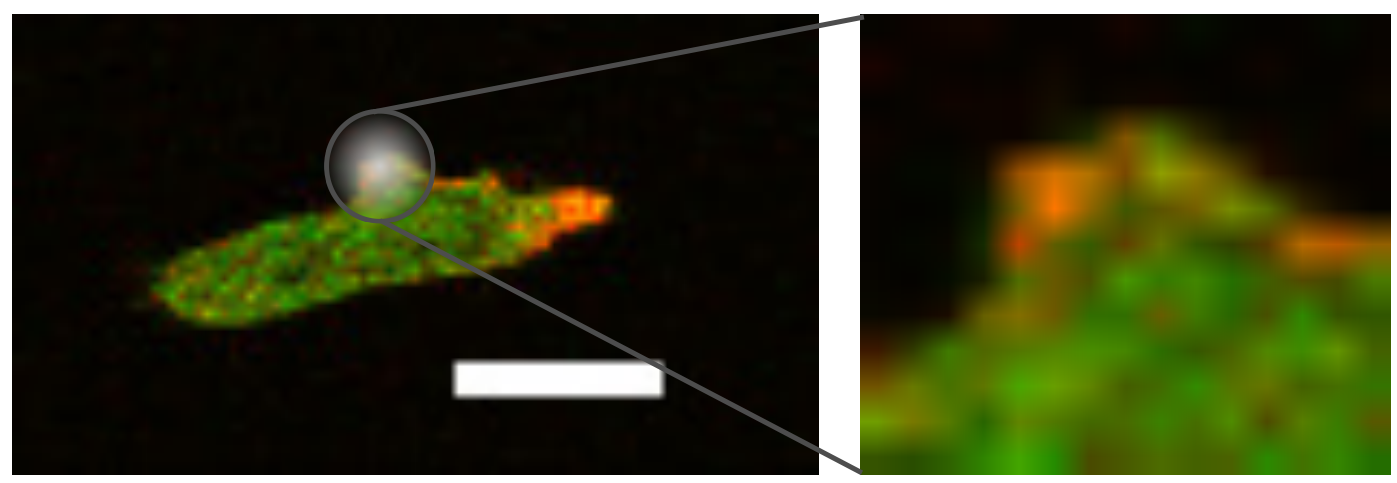

Figure 5.1: Zoom on overlay of two colors channel real raw data to visualise the pixel at cell membrane. Both fluorescent labels are visualized by a false-color grey scale and afterwards overlayed, where green corresponds to the Raf1-RBDGFP and red corresponds to LimE-mRFP. The raw data consists of grey scale pixels. For the visualisation we include colours of different intensity depending on the intensity measured by the photo diode of the microscope and saved in the data image. Hence the cell outlines in the raw data is defined by bright pixels close to darker pixels. To extract a smooth cell outline from this discrete pixels with eventually strongly different intensities we use an active contour algorithm based on (22). Scale bar corresponds to $10 \mu \mathrm{m}$.

cytoskeleton. Hence, we find for each position around cell circumference values of the fluorescence intensities. A commonly used way to plot values like this with a time dependancy are kymographs. The values for curvature and fluorescence intensity are incorporated in two-dimensional maps, in which one axis corresponds to the position around the cell circumference, while the second axis corresponds to the time position. The y-axis corresponding to the position on the cell circumference is normalized by the arc length of the cell circumference, as its length can change over time. Hence we choose a specific amount of points (200) and interpolate such that we have equally spaced points around the whole circumference. This results into a range of the $y$-axis curves from 0 to 1 , where 1 corresponds to the length of the cell circumference. In such a way one can see in these diagrams the full spatio-temporal 


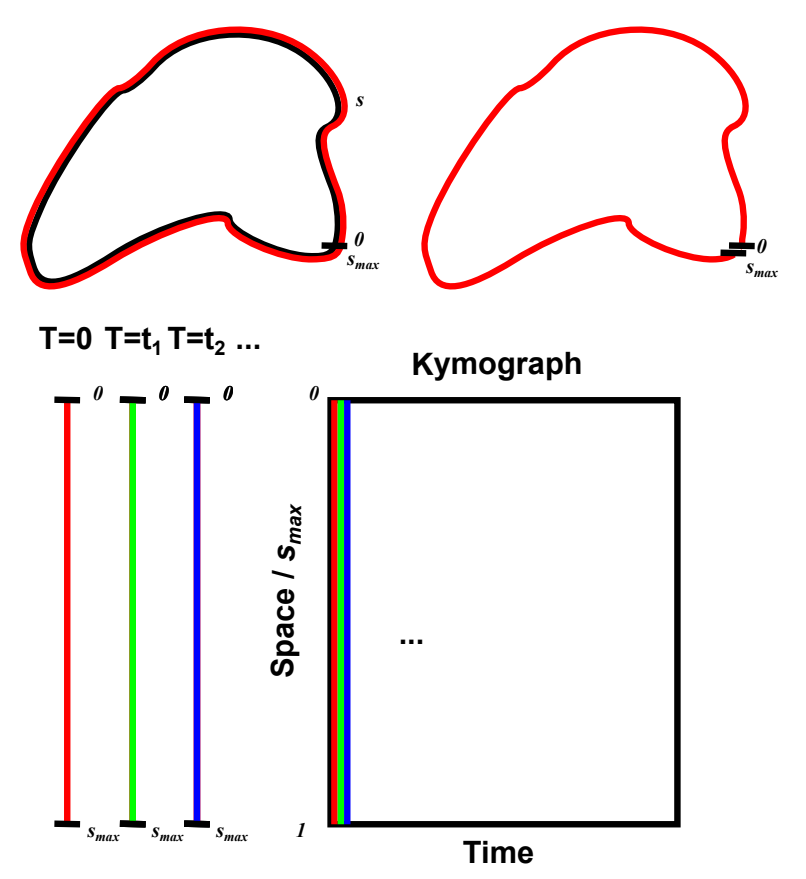

Figure 5.2: Sketch of the kymograph construction. For the construction of the kymograph we extract from every frame of the time series the data around the whole cell circumference (curvature or fluorescence intensity). Afterwards this signal is plotted as a line into a map, where the y-axis corresponds to the position around the cell perimeter. The data of the next timestep is plotted next to it. This results in a map where the $\mathrm{x}$-axis corresponds to the time and the $y$-axis to the position around the cell. To have the same amount of points in each time step we divide the cell circumference into a constant number of points in every frame.

behavior of the signal. Particularly changes in shape or intensity are visible in these graphics.

Additionally we have to consider the effect of photobleaching, described for instance by Song and coworkers in (70). Due to illumination a fluorophore may be destroyed and stop to emit fluorescent light. This effect should be decreased in living cell fluorescence microscopy, as, due to the genetic labeling, the cell is able to produce the fluorescent label. If the rate of photobleaching is higher than the rate of production of the fluorescent protein inside the cell, the fluorescent signal will 
decrease over time. This effect is significantly smaller in the the experiments using CLSM than for the experiments performed with with the sdCLSM. To correct for this effect, we use a linear detrending of the data in time, where we calculate the mean value of each timestep, use these mean values for the linear detrending and substract the detrended mean from the fluorescent signal at each timestep.

\subsubsection{Spatiotemporal Cross-Correlation}

A reasonable way to calculate dependencies of two spatiotemporal signals as the kymographs from the contour analysis are two-dimensional cross-correlations. Those spatiotemporal cross-correlation coefficients can be calculated by the following expression that is basically an extension of the one-dimensional Paerson correlation coefficient. If we have to signal $f(x, y)$ and $g(x, y)$, we can calculate the correlation coefficients for shifts $\tau, \rho$ in both variables $x, y$ with the equation:

$$
r_{f, g}(\tau, \rho)=\frac{\sum_{x, y}(f(x, y)-<f>)(g(x+\tau, y+\rho)-<g>)}{\sqrt{\sum_{x, y}(f(x, y)-<f>)^{2} \sum_{x, y}(g(x, y)-<g>)^{2}}} .
$$

For ideal agreement of the signals, we would find a correlation coefficient $r_{f, g}(0,0)=$ 1 and decreasing values for other values of $\tau$ and $\rho$. As an example, we show a curvature kymograph and its two-dimensional cross-correlation map in Figure 5.3. 

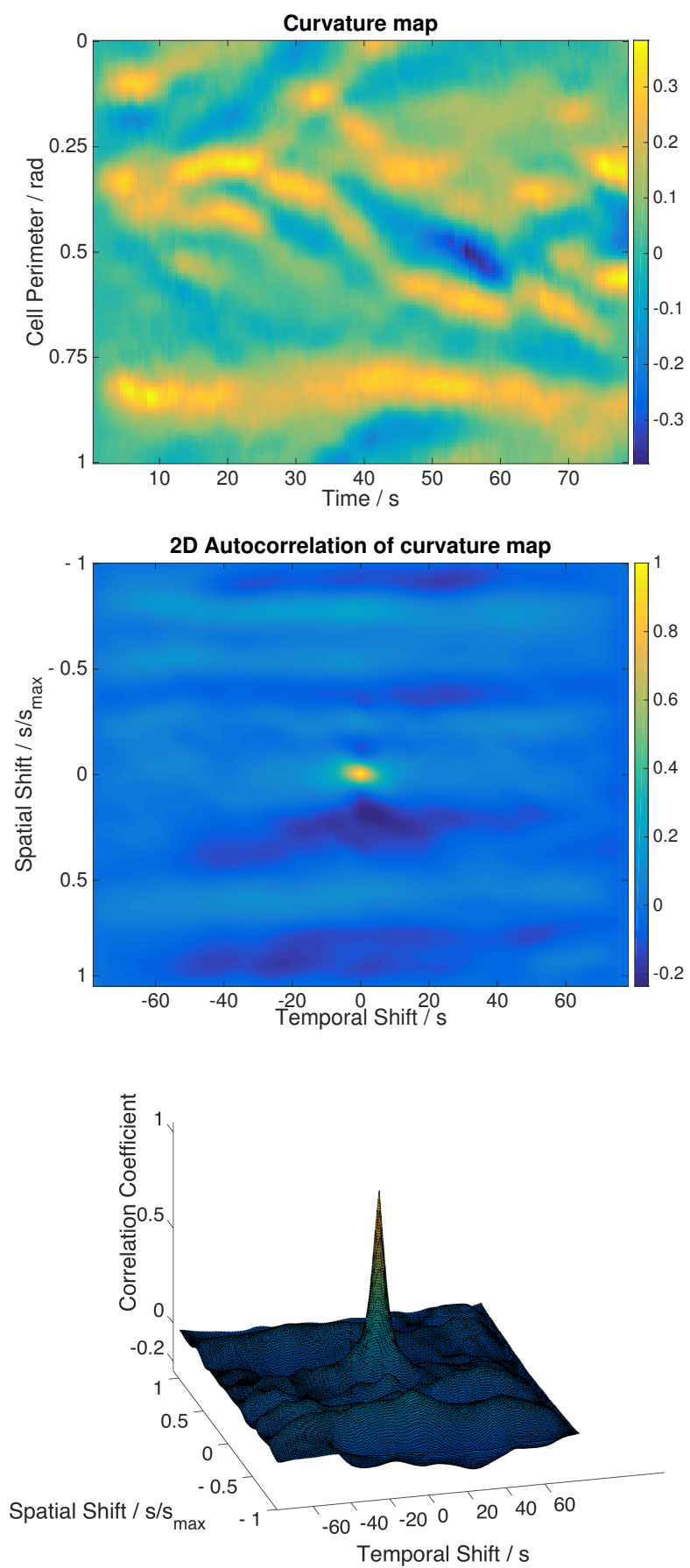

Figure 5.3: Sketch of principle of two-dimensional cross-correlation. On the right hand side we show a kymograph of the result data (see 5.5). The graphs in the middle and on the right both show the two-dimensional autocorrelation of this kymograph, once with a two-dimensional color plot and once with a three-dimensional surface plot. In the very center we find a narrow peak that has the maximum at one. 


\subsection{Results of Confocal Laser Scanning}

\section{Microscopy}

In this section we will describe the results of the experiments performed with the Olympus FV10 Confocal Laser Scanning Microscope (CLSM). We will divide the results of this setup into whole cell data, where we are using the full kymograph gathered, and splitting pseudopodia, for which we extract the events of curvature change out of the kymographs.

\subsubsection{Results}

The analysis of the coupling of geometry, signaling cascade and the actin cortex begins at the level of the whole cell. After labeling the $D$. $d$. cells with the two labels, Raf1-RBD-GFP as a label for Ras-G and LimE-mRFP as label for filamentous actin, we starve them for six hours according to the pulsing protocol described in section 3.1. These cells are very motile and chemotactically active after the pulsing. They were placed in a microfluidic gradient mixer, as shown above in subsection 3.2.3.2. in which they are exposed to a linear, stable and well-controlled chemoattractant gradient. The imaging process requires to image one or a few cells to be placed in a rather small imaging area. We choose the imaging area as small as possible to achieve a high temporal resolution, i.e. minimizing the scanning time of the CLSM. Yet it has to be large enough for the cell to stay inside during the imaging time. Hence, we have to find a compromise in the size of the imaging area.

After we defined the $\mathrm{x}$ - and $\mathrm{y}$-position of the imaging area, we have to specify the z-position. As we want to investigate the influence of the signaling protein Ras, 
we choose the z-position in such a way that the intensity of the Raf1-RBD-GFP label is maximal. For most of the cells, this leads to imaging areas that are slightly above the surface $(1 \mu \mathrm{m}$ to $2 \mu \mathrm{m})$ of the glass slide. An exemplary time series of a splitting pseudopod is shown in Figure 5.4. A $D . d$. cell with the above mentioned labels is visualized during the whole splitting process of a pseudopod inside the microfluidic gradient mixer with the concentration gradient of $0.4 \frac{\mathrm{nM}}{\mu \mathrm{m}}$ towards the top of the images. As the imaging is done with a oil immersion 60x objective (Olympus UPLSAPO 60x), we were not able to measure the position of the cell in the gradient, as we would have to use smaller magnification for that. In the beginning of the shown series at the very right of the cell high fluorescent signals are visible as well as in top middle. The high fluorescence intensity on the right hand side is due to a pseudopod, while the higher fluorescence in the center is a developing pseudopod in the following. At $T=4.53 \mathrm{~s}$, this pseudopod has already formed and is the only position where a high fluorescence signal can be found. Starting with the third image with time stamp $T=9.06 \mathrm{~s}$, the pseudopod splitting event becomes visible. Until $T=18.12 \mathrm{~s}$ both new pseudopods are growing and show high fluorescence intensity in both channels. In contrast, at $T=22.65 \mathrm{~s}$, the fluorescence at the pseudopod on the right-hand-side is not above the average anymore. This pseudopod will be retracted subsequently, while the one at the left hand side proceeds growing until $T=36.42 \mathrm{~s}$.

The microscopic image series like the one shown above in Figure 5.4 are analyzed as described in section 5.2.1 by an active contour algorithm to extract the cell circumference as well as the curvature. To correlate this geometric property of the cells with the signaling protein Ras- $\mathrm{G}$ and the actin cortex, we define a mask for the cell cortex based on the extracted cell circumference. Within this mask, 

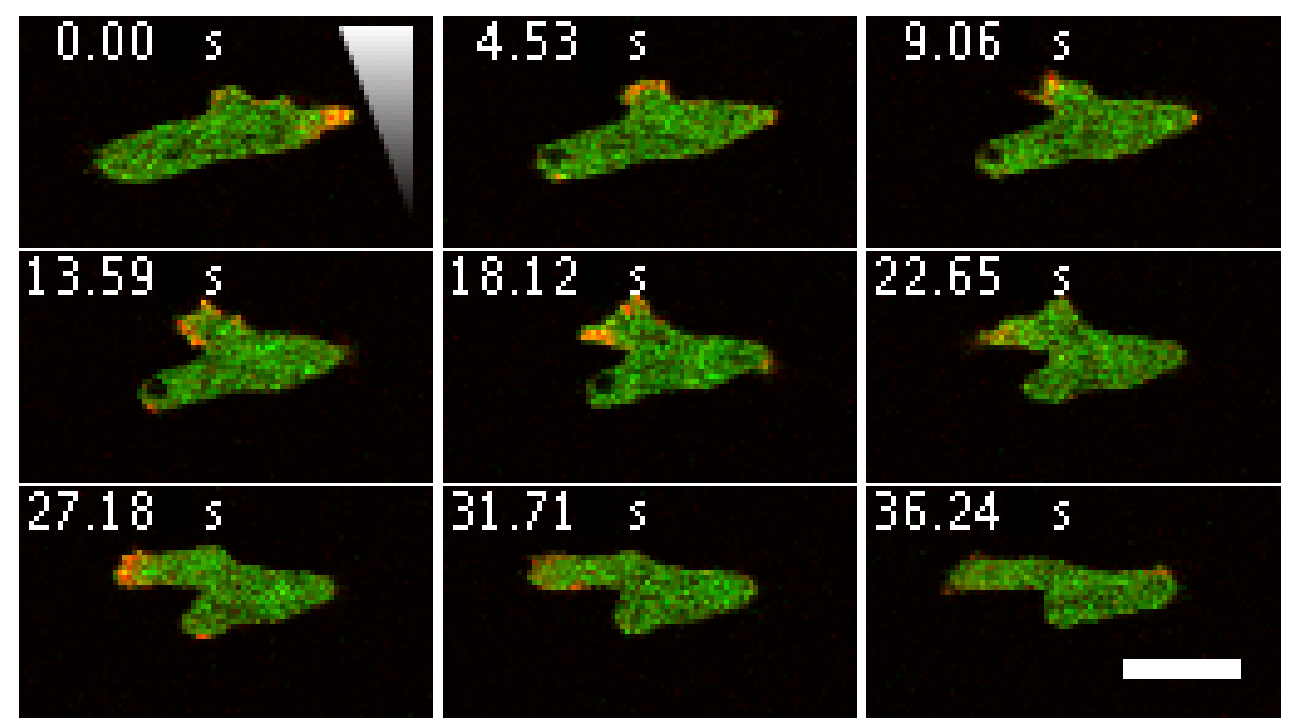

Figure 5.4: A single cell showing a pseudopod splitting. Cell migrates in a microfluidic gradient mixer with a gradient of $0.4 \frac{\mathrm{nM}}{\mu \mathrm{m}}$ that is to the top. Time between the shown images is $4.53 \mathrm{~s}$. Scale bar corresponds $10 \mu \mathrm{m}$. The green colour is a colour representation of the grey scale fluorescence signal of Raf1RBD-GFP and the red colour accordingly represents the fluorescence signal of LimE-mRFP, both in arbitrary units. At the upper left one can read a time stamp in seconds. The fluorescent signals show maxima at the leading edge of pseudopods for both channels.

we extract the fluorescence intensities of both channels. Hence, we can construct kymographs for every frame of the three time series. An example of these results is shown in Figure 5.5. The top figure shows the curvature of a migrating $D . d$. cell in a microfluidic gradient mixer. The curvature is shown for about one and a half minute. At the cell perimeter of around $0.75 s_{\max }$, the back side of the cell is visible. The positive curvature of the convex cell back is virtually constant over the whole imaging time. There are few changes in the geometry at the back, as expected. On the upper part of the Figure the strong dynamical changes at the leading edge of the cell is clearly visible. Various splits of the high curvature areas can be seen. On the second image, the fluorescence intensity of the Raf1-RBD-GFP is shown. The 
fluorescence intensity kymograph has a similar structure to the curvature kymograph. At the cell back there is no strong signal of the Ras protein. In the upper part, this is significantly different. There are, as for the curvature signal, various splitting events. In a detailed examination it becomes apparent that the higher intensities for the Raf1-RBD-GFP signal are located closely to the highest curvatures. To be more precise, the strongest signals are located at the locations of the highest curvatures. The fluorescence signal of the LimE-mRFP kymograph shown in Figure 5.5 at the bottom differs from the other two kymographs. In this case, the high fluorescence patches are much smaller and less branched. Apparently the process of actin polymerization is a process that is acting in a local way and there are huge differences between high intensity patches and low or no intensity regions.

\subsubsection{Curvature Dependence on the Fluorescent Patches}

We investigate the dependence of the curvature on protein activity, visualized by fluorescence of 31 D. $d$. cells (AX-3 WT with Raf1-RBD-GFP and LimE-mRFP labels) inside microfluidic gradient mixer with a concentration gradient of $0.4 \frac{\mathrm{nM}}{\mu \mathrm{m}}$. Hence we isolate the fluorescent patches by a thresholding of the kymographs. We define the fluorescent patches as all areas where the fluorescence intensity is higher than a percentage of the maximal fluorescence intensity of the normalized signal. We start with $10 \%$ of the maximal fluorescence and increase the value up to $90 \%$. In the following graphs, we investigate the influence of the threshold on the distributions of the two other signals. In Figure 5.6, we show the different distributions of the curvatures at Ras and LimE patches on the upper images, while in the second row the mean values of the fluorescence intensities are shown in dependence of 

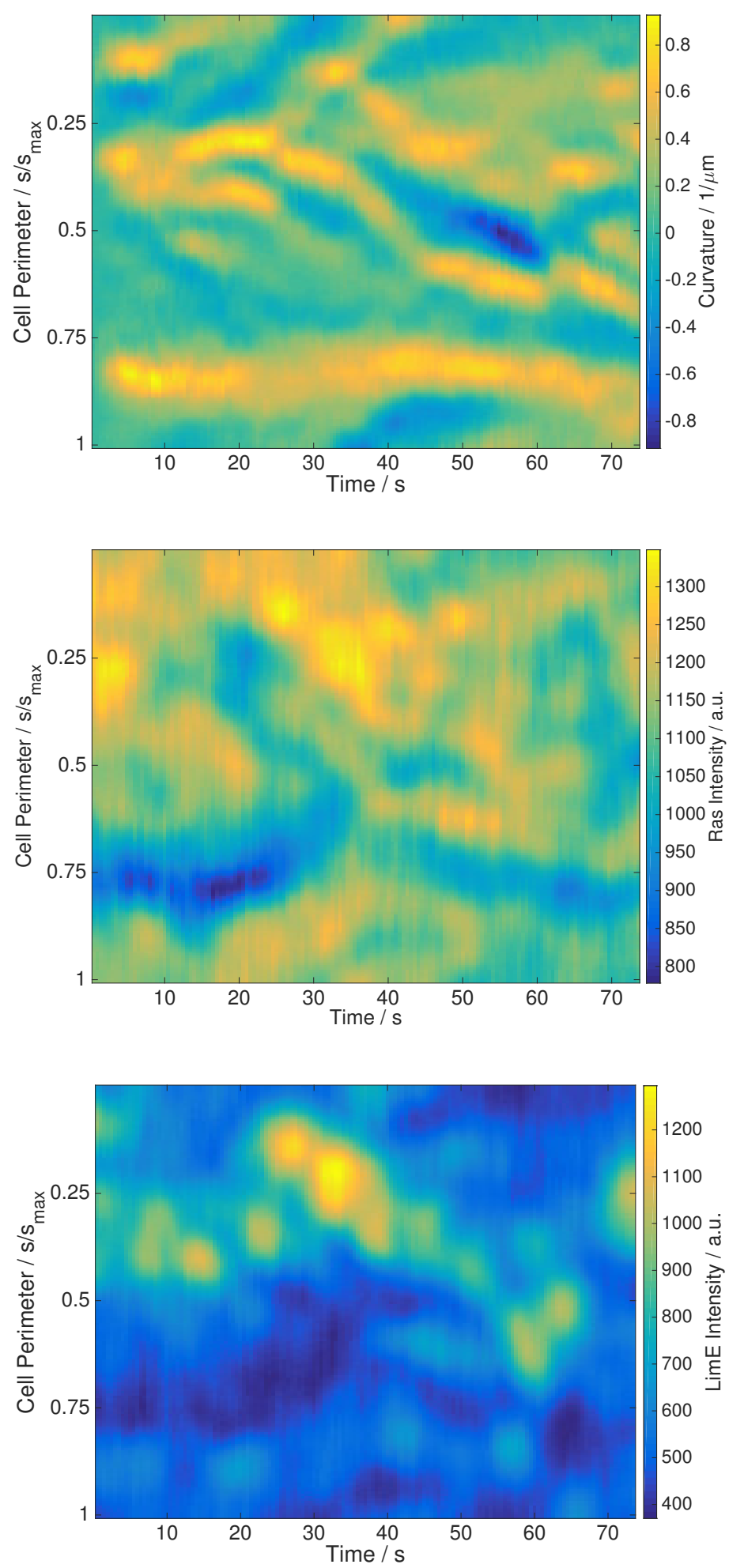

Figure 5.5: Kymographs of Curvature, Ras-Fluorescence and LimE-Fluorescence of an exemplary cell. On the curvature kymograph we can see a very dynamic change of the curvature due to shape changes on the leading edge, while on the bottom the retracting back is very uniform. On the kymograph in the middle we visualize the fluorescence intensity of Ras-G via Raf1-RBD-GFP. Here we can find strong patches at the upper part, while close to the retracting back there is a minimum of intensity. Qualitatively this is similar on the lower kymograph of the LimE-GFP fluorescence intensity, which corresponds to actin polymerization. The patches of high fluorescence activity are much more confined than for the case of Ras. 
the patch threshold of the other label. The errorbars correspond to the standard deviation of the distribution. A general feature of all four graphs is that the mean curvature as well as the fluorescence intensities increase with increasing threshold of the fluorescent patches. In addition we find that the errors increase for the last two points at thresholds of 0.8 and 0.9 . The increasing standard deviations are due to the decreasing amount of points. The mean value of the LimE fluorescence intensity at Ras patches seems to saturate. As we find a steady increase of all curves and only for the LimE fluorescence at Ras patch a saturation at a threshold of 0.7, we decided to use this threshold value for both fluorescent labels. The fact that the signals monotonously increase with higher patch threshold, shows a strong correlation of the three signals, Ras, LimE and curvature. The saturation of LimE fluorescence at Ras patches can be explained with the stronger localization of LimE fluorescence, even at Ras patches with less high intensity the full LimE patch is already found.

At these patches (defined by $70 \%$ of the maximal intensity), we investigate the curvature values. We can immediately see that the distribution of the curvature values at Ras patches is shifted as well as skewed. The distribution of all curvature values is shown in the bottom graph of Figure 5.7, while the distribution of curvature values at LimE-mRFP and Ras-GFP patches are shown above. The distributions can be fitted reasonably well by lognormal distributions, which are also shown in Figure 5.7 .

The lognormal distribution is defined by

$$
f(x)=\frac{1}{x \sigma \sqrt{2 \pi}} \exp -\frac{(\ln x-\mu)^{2}}{2 \sigma^{2}}, x>0 .
$$

To fit our curvature distribution with a lognormal distribution, we have to shift the data to positive values, as the lognormal distribution is only defined for positive 
values. After the fit, we have to shift the fit and the data back to its original values.

We find the mean of all curvature value to be $0.176 \frac{1}{\mu \mathrm{m}}$, while the mean of the fit is $0.177 \frac{1}{\mu \mathrm{m}}$. The standard deviation of the data is $0.238 \frac{1}{\mu \mathrm{m}}$ while the standard deviation of the fit is $0.253 \frac{1}{\mu \mathrm{m}}$. The main properties are reasonably well reflected by the fit.

The parameters of the fit of all curvature values, without any dependence on the fluorescence, are

$$
\begin{aligned}
& \mu=0.2481[0.2478,0.2484] \text { and } \\
& \sigma=0.1918[0.1916,0.1921],
\end{aligned}
$$

where the $95 \%$ confidence intervals are shown in brackets. The mean value of the distribution is $0.176 \frac{1}{\mu \mathrm{m}}$ while the fitted curve has a mean at $0.178 \frac{1}{\mu \mathrm{m}}$. To compare the fitted distribution with the data of the histogram, we investigate the mean value and the standard deviation of both distributions. The curvature distribution at Ras patches is shown on the graph in the middle of Figure 5.7 and the fit parameters are shown below:

$$
\begin{aligned}
\mu_{\text {Ras }} & =-0.62[-0.64,-0.60] \text { and } \\
\sigma_{\text {Ras }} & =0.57[0.56,0.59] .
\end{aligned}
$$

The parameters of the fit are in good agreement, the mean of the data being $0.48 \frac{1}{\mu \mathrm{m}}$ and the mean of the fit $0.51 \frac{1}{\mu \mathrm{m}}$. The same holds for the standard deviations, where the std value for the data is $0.26 \frac{1}{\mu \mathrm{m}}$ and the value for the fit is $0.39 \frac{1}{\mu \mathrm{m}}$.

The curvature distribution at the LimE patches is fitted with a lognormal distribu- 
tion with the following parameters:

$$
\begin{aligned}
\mu_{\text {LimE }} & =0.080[0.077,0.082] \text { and } \\
\sigma_{\text {LimE }} & =0.268[0.266,0.269] .
\end{aligned}
$$

We find that both values are in very good agreement as the mean value of the data is $0.434 \frac{1}{\mu \mathrm{m}}$ and the mean of the fit is $0.439 \frac{1}{\mu \mathrm{m}}$ and as well the standard deviation of the data is 0.265 and the standard deviation of the fit is 0.306 .

The distributions of the curvatures at the patches are both in good agreement for both the mean values and the standard deviations. The two distributions differ from the distribution of all curvatures. The mean value of the he distribution of curvatures is $0.18 \frac{1}{\mu \mathrm{m}}$. The mean of the distribution of the curvature values at Ras-Patches is at $0.48 \frac{1}{\mu \mathrm{m}}$ and hence at a value more than twice as big as for all curvatures. The distribution of curvature values at LimE patches is peaked at similar high value at $0.43 \frac{1}{\mu \mathrm{m}}$. Hence we can tell that the curvature is higher at the spatial positions where the fluorescence intensity is high. 


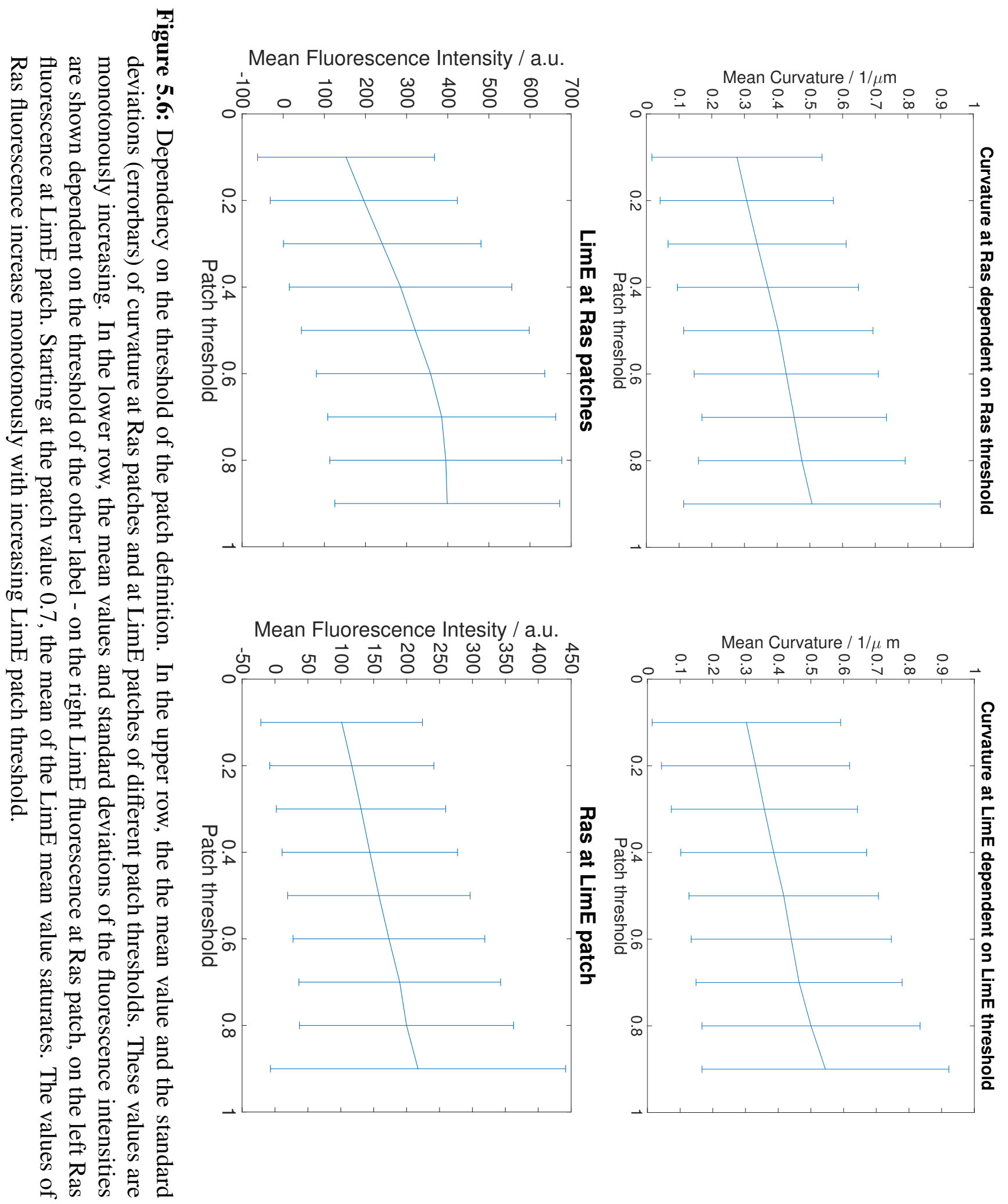



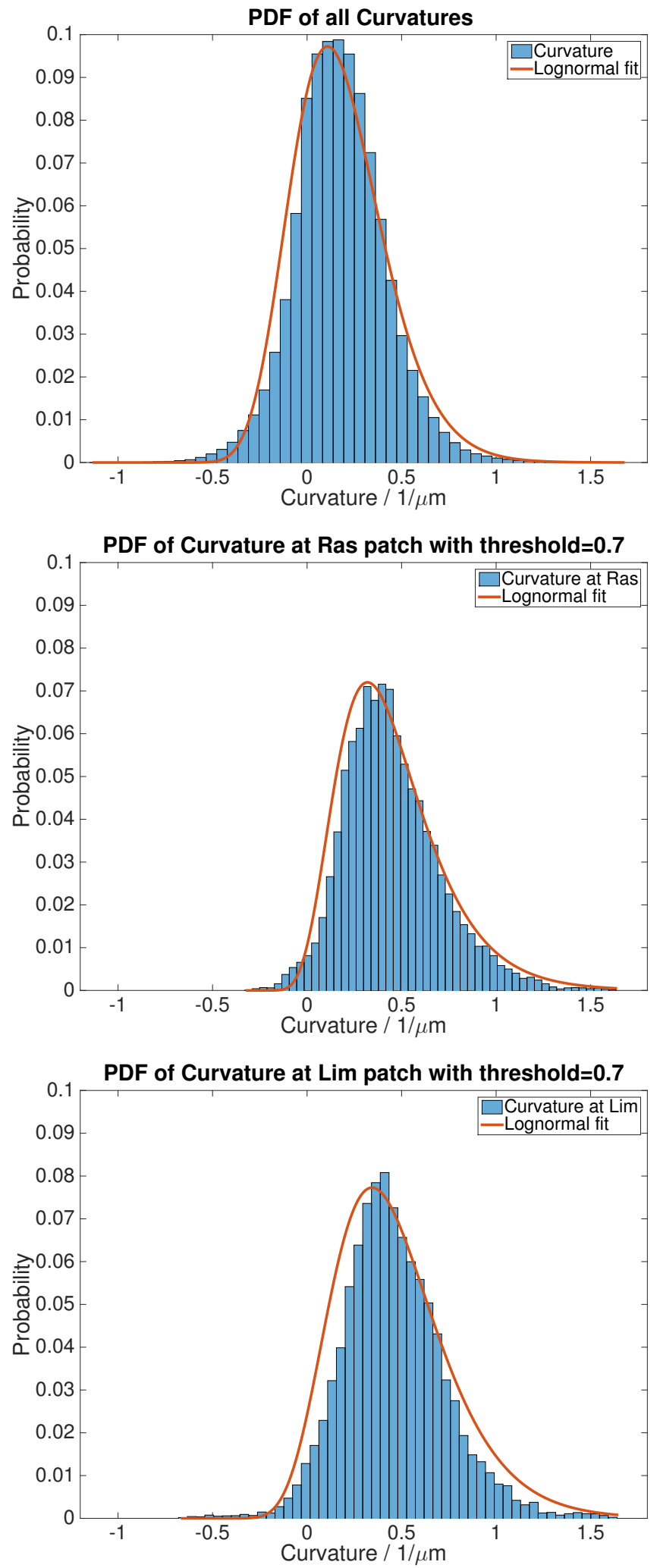

Figure 5.7: Distributions of curvature. On the upper graph, the distribution of all curvature values is shown. The red line corresponds to the lognormal fit. The distribution of the patches of high Ras intensity is shown in the graph in the middle. The graph at the bottom shows the distribution of the curvature values at LimE patches and the lognormal fit. The distribution of the curvatures at fluorescent patches are both strongly shifted compared to the distribution of all curvatures. 


\subsubsection{Cross-Correlations of Kymographs}

We investigate the cross-correlations of these signals as two-dimensional crosscorrelation functions of the kymographs. Hence, the cross-correlation functions can tell us details about spatio-temporal shifts of the data signals. We are interested in spatial shifts, as they may provide details of the geometrical distribution of the proteins on to the cell circumference. The temporal shift may lead to a deeper understanding in the processes of protein recruitment and pseudopod formation, as it can help to distinguish a cause-and-effect chain.

To obtain results of the cross-correlation that include more than one cell, we calculate the cumulated cross-correlation function of various cells (31 WT Raf1RBD-GFP+LimE-mRFP). To achieve this, we resample the data in terms of the time steps in such a way that all experiments we compare have the same time step between to frames, namely $t=0.5 \mathrm{~s}$. Then we average the cross-correlation functions. We use the temporal data of $\pm 15 \mathrm{~s}$ time-shifts. In Figure 5.8, those two-dimensional cumulated cross-correlation functions are shown. As described in section 2.3.1 the current understanding of the chemotactic signaling cascade suggests that the activity of Ras-G should activate the actin polymerization and that this polymerization is responsible for the morphologic change of the cell.

In Figure 5.8 the cumulated cross-correlation functions of curvature, Ras and LimE are shown. In the top row, the cross-correlations of curvature and Ras is shown, visualized in three different ways. On the right graph, the cross-correlation is plotted as a color plot and in the middle the same data is plotted as a colored surface plot for easier visualization. On the left graph, we show a measure of asymmetry. To calculate this measure, we substract the values of positive direction 
(right part of the cross-correlation function) from the negative direction (left part of the cross-correlation function). Hence, a small value this measure corresponds to a small asymmetry, while a high positive value corresponds to larger values in the negative direction and a high negative value corresponds to larger values in the positive direction.

The results of the series of experiments performed with 31 migrating cells inside a microfluidic gradient mixer using a conventional CLSM. The cross-correlation coefficients are maximal at the no-shift position for all three cases. The values for the maximal cross-correlation is medium in the two cases where curvature is involved (0.4), but rather high (0.7) for the correlation of the two fluorescent labels. The cross-correlation is very strongly dependent on spatial shift and confined in an area of small shifts. This is also visible in the three-dimensional surface plots. If we focus on the temporal axis of the correlation functions we realize very clearly a shift towards negative time for the cross-correlation functions of curvature and Ras, respectively LimE, we find a different behavior to the cross-correlation function of Ras and LimE. We find the shift towards negative times of about one quarter of the maximal cross-correlation coefficient for curvature and Ras as well as for curvature and LimE. In the case for the cross-correlation of Ras and LimE, there is no shift visible, even though the cross-correlation is very high. These shifts found for the cross-correlations of curvature and Ras, respectively curvature and LimE, imply that curvature is lagging behind the biochemical signals in the cell, hence the hypothesis that the chemotactic signaling cascade is responsible for the cell geometry change is strengthened by this finding. 

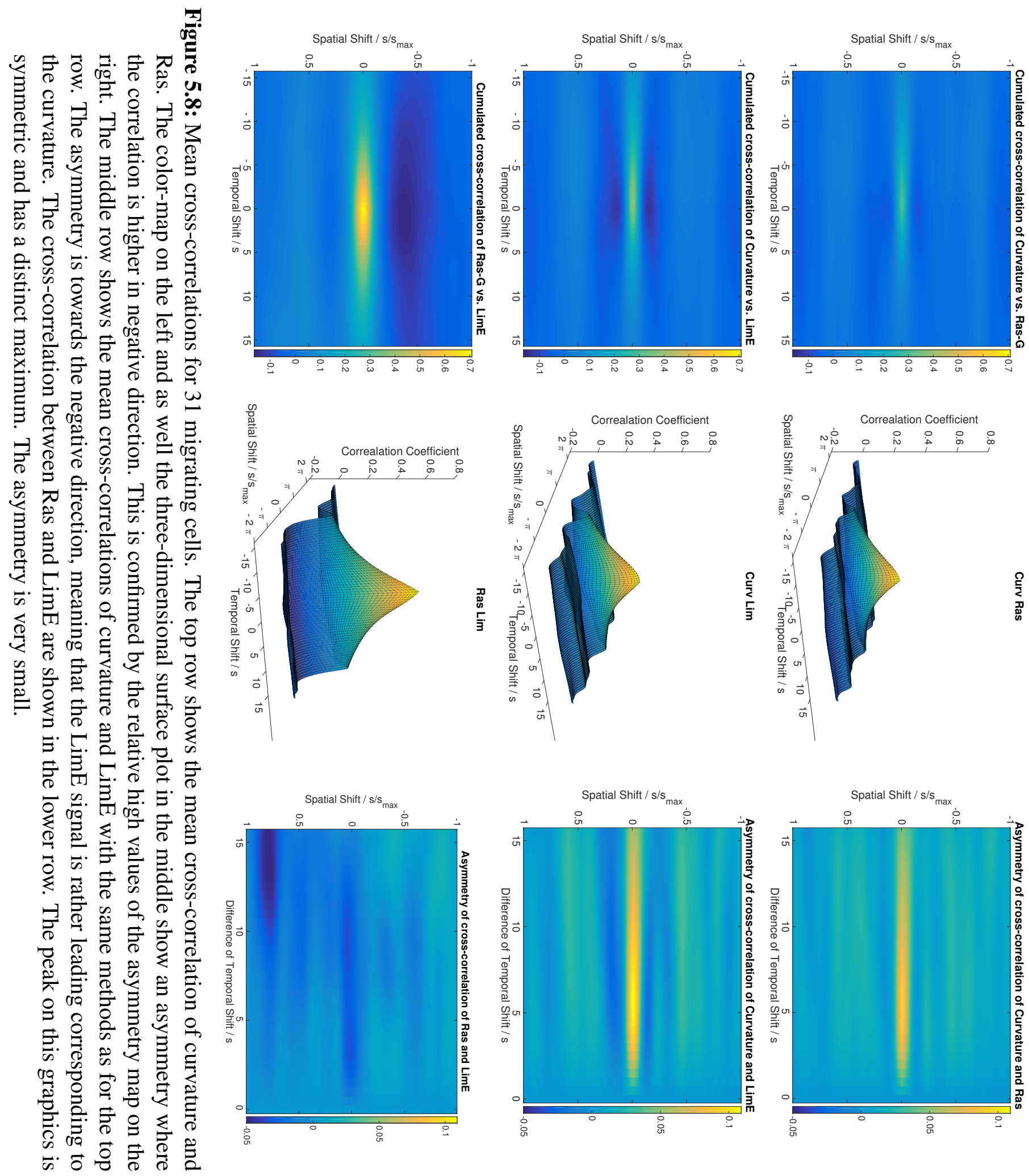


\subsubsection{Cross Correlations at Pseudopod Level}

In the kymograph analysis of the whole cell, the curvature at the cell back is always positive, but, as it is the cell back, no fluorescence activity is found. The highly positive curvature at the retracting back faces no fluorescence signal. Hence the cross-correlation of the kymograph decreases. As we are interested in the dynamics at the leading edge and especially in the formation of pseudopodia, we will now investigate the splitting of pseudopodia in greater detail. Therefore, we also choose to do the cross-correlations on the level of single pseudopods at their splitting events. From the kymographs of the aforementioned 31 migrating cells, we could extract 121 pseudopod splits.

To define a splitting event, we are using the curvature kymograph. In a spatiotemporal neighborhood where a positive curvature (convex part of the cell - hence a propulsion) splits into two positive curvature areas and a negative curvature area in between, we will define as a pseudopod split. The investigation shows that in order to characterize the whole process in this way, we need to define these neighborhoods spatial extension as a quarter of the total cell outline. The temporal extension of the pseudopod split is chosen to be $15 \mathrm{~s}$. This choice is reasonable in concern to the measured data and also suitable compared to the usual pseudopod extension time which is known from the literature to be $12 \mathrm{~s} \pm 5 \mathrm{~s}(\underline{13})$.

Regarding the images of the mean cross-correlation functions, we observe several properties. The property of most importance is that there is asymmetry towards negative time. This finding strongly indicates that the biochemical signaling cascade is responsible for pushing out the membrane leading to a higher membrane curvature (pseudopod). This result does not show evidence for the hypothesis, that the curved 

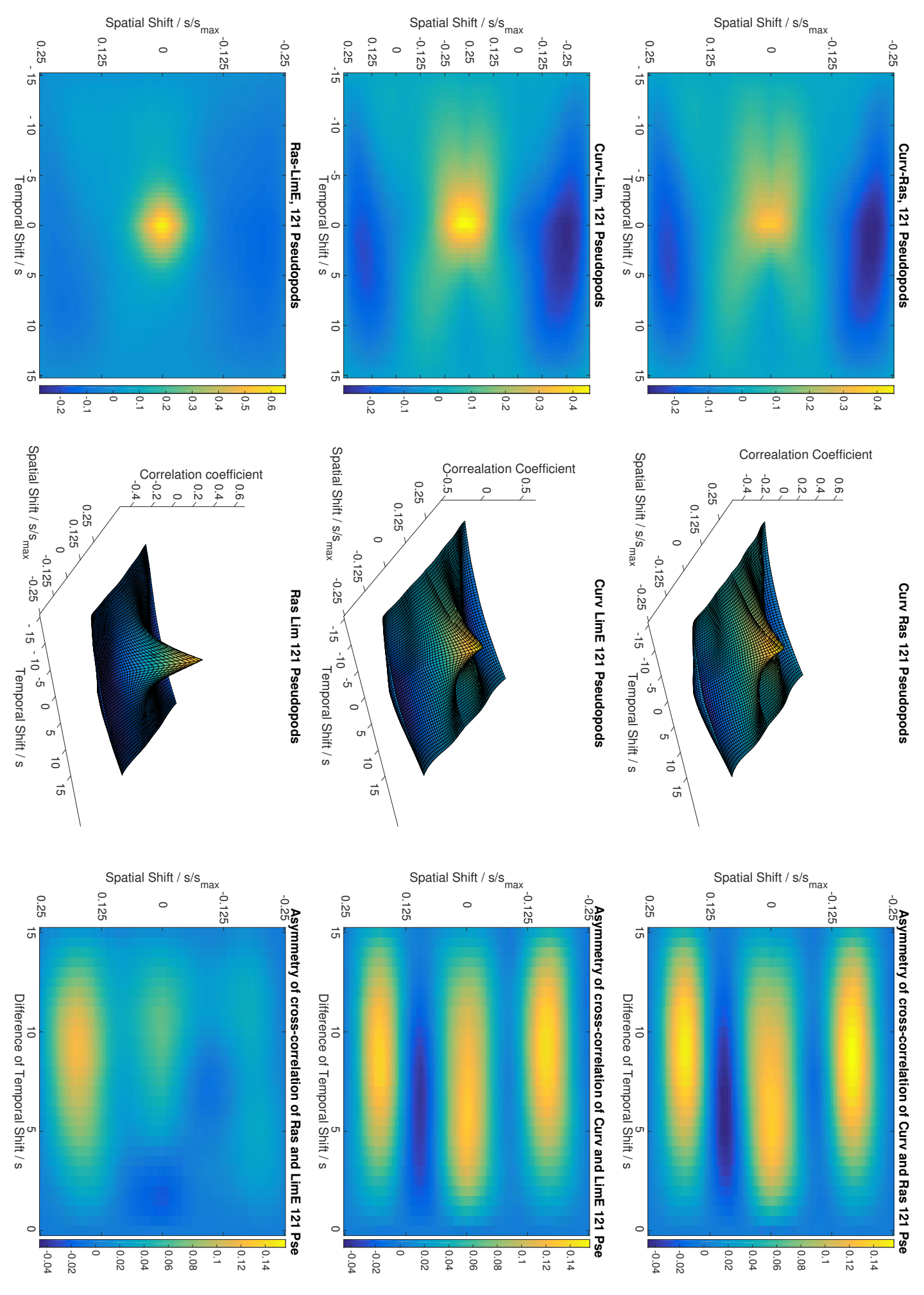
membrane is the origin of pseudopod formation. Additionally, we find that there regions of small positive correlation slightly shifted in space, as well in negative time shifts as even smaller for positive time shifts. This spatial shifts correspond to the geometry of the splitting pseudopodia. Out of one positively curved region, there will be two positively curved regions with a negatively curved part in between of these two. The results show that the spatial dependence is higher for negative times and there cross-correlation is higher. The results for the cross-correlations of both fluorescent signals with the curvature are very similar.

In contrast to this, the cross-correlation of the two fluorescent intensities is a very uniform symmetric function with a peak at no-shift position, indicating that the signals are very similar. Within this analysis we are able to tell that Ras and LimE are visible before the curvature, but we can not tell a temporal difference between Ras and LimE. To answer this important question in our analysis of the problem, we started to increase the temporal resolution. 


\subsection{Fast Spinning Disc Confocal Laser}

\section{Scanning Microscopy}

As all the results from the ordinary CLSM showed the strongest correlations without any temporal and spatial shifts, we start to perform experiments with a higher temporal resolution. To specify whether this is an effect of the low time resolution of $0.5 \mathrm{~s}$ we decided to increase the temporal resolution by changing the experimental setup to a spinning disc CLSM (sdCLSM). The frame rate of the sdCLSM was in most of our experiments 16.8979 frames per second. Besides the eightfold incrase of the frame rate, also the imaging area is increased and hence it is possible to image several cells similarly.

\subsubsection{Results for Wildtype Cells}

In this section we will similar to the results of section 5.3 analyze the spatiotemporal dynamics of $D . d$. wild type cells first at the whole cell level and then at the pseudopod level. We use the same cell line (AX3 WT Raf1-RBD-GFP+LimE-mRFP) and the same microfluidic setup as in the section before (microfluidic gradient mixer with a concetration gradient of $0.4 \frac{\mathrm{nM}}{\mu \mathrm{m}}$ ) and investigated 46 cells in total.

\subsubsection{Resolution Increase Due to Setup}

To show the immensely increased temporal resolution in Figure 5.10, the curvature and the Ras-G kymograph of an experiment with the sdCLSM is plotted on top of a zoom-in of the Figure 5.5 with the same length in time. The curvature map in the top graph possesses much more details than the map from the bottom. This is even 
more pronounced in the case of the Ras-G kymograph. The increase of the temporal resolution is the main aspect we were aiming for and in Figure 5.10, we see two curvature kymographs where in the one extracted from conventional CLSM (low graph) the frame rate is 2 frames per second, while in kymograph of the sdCLSM (top graph) the frame rate is 16.8979 frames per second. Hence we reach more than an 8-fold increase of the temporal resolution by using the sdCLSM. Furthermore the sdCLSM setup in the Enderlein Departement at University of Göttingen possess a “100x UPlanSApo, 100x magnification, NA 1.4, Olympus” objective, leading to an additional increase of the spatial resolution. At the conventional CLSM we are able to reach a pixelsize of $207 \mathrm{~nm}$ (in Figure 5.10 it was $414 \mathrm{~nm}$ to increase the temporal resolution), while for the sdCLSM a pixelsize of $133 \mathrm{~nm}$ is reached. Additionally we do not have to reduce the region of interest of the confocal scan for the sdCLSM, hence we are able to image more cell at a time. 

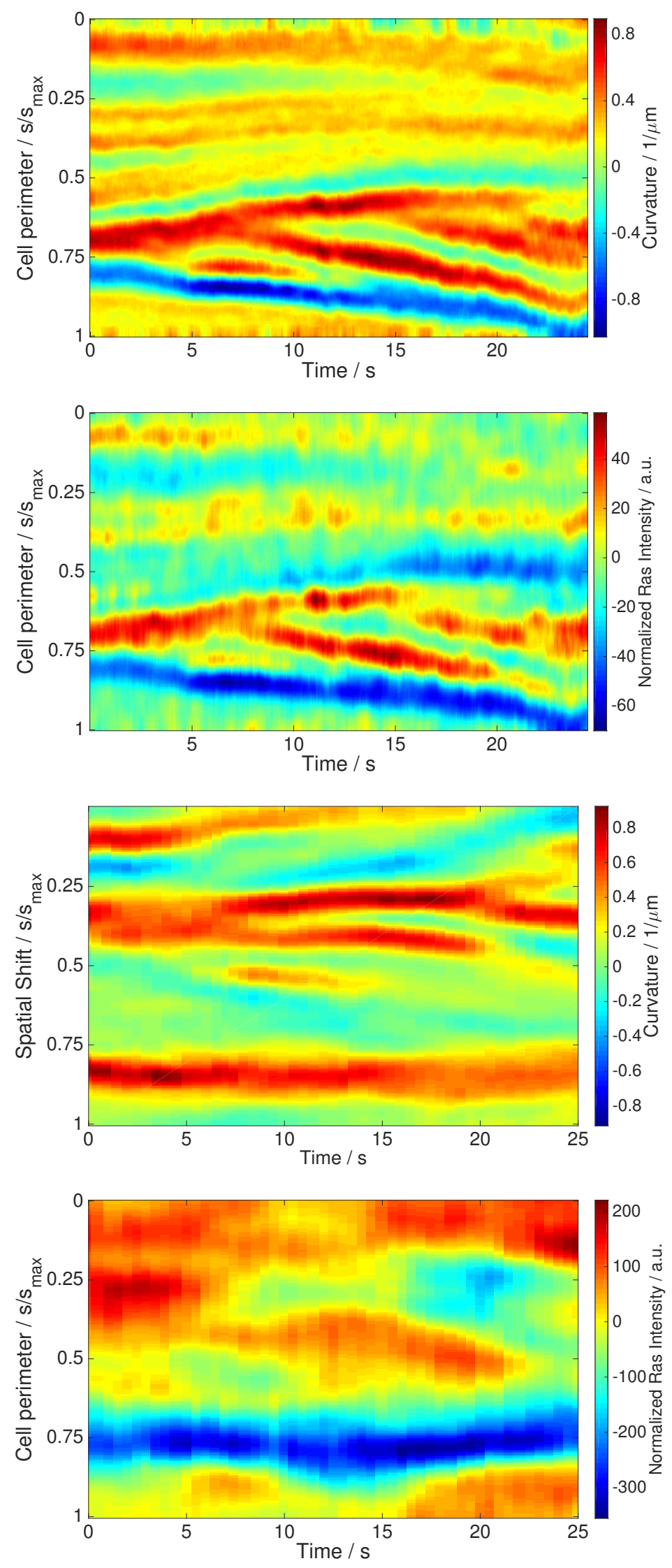

Figure 5.10: Curvature and Ras kymographs of different WT cells with sdCLSM and CLSM. Top images shows a curvature and a Ras-G activity kymograph of a WT cell with double label of Ras-GFP and LimE-mRFP imaged with sdCLSM at frame rate of $16.8979 \mathrm{~Hz}$. On the bottom images a curvature and a Ras-G activity kymograph of another WT cell is shown for the same time as the top image with frame rate $2 \mathrm{~Hz}$. 


\subsubsection{Dependence on Fluorescence Patches}

As for the conventional CLSM we also analyze the dependencies of the distributions of the lognormal fits of curvature and fluorescence intensities on the fluorescent patches. The setting is exactly the same as before in subsection 5.3.1.1, besides the higher resolution due to the sdCLSM setup.

The curves on Figure 5.11 are all monotonously increasing for increasing value of the patch threshold. This holds for the curvature distributions for both conditions (Ras patch or LimE patch) and as well for the distributions of the fluorescence intensities conditioned of the other fluorescent patch. In the case of the Ras patches, it seems that there is a change in the small change in the steepness of the curve at a threshold of 0.7 in for the curvature distribution and for 0.8 for the LimE distribution. There is no plateau in the plot for the LimE distribution at Ras patches.

In addition, we realize in general, but especially for the LimE intensity at Ras patches, that the standard deviations of the fitted curves are bigger than for CLSM data presented in Figure 5.6. This may be caused by the smaller number of cells and especially by the strongly reduced number of pseudopods due to the measuring technique as the imaging time per cell is drastically reduced.

\subsubsection{Cross-Correlations of Kymographs}

The kymographs shown in Figure 5.10 with high temporal resolution will be investigated as in the preceeding section with the two-dimensional cross-correlation. As result we gain the following graphs shown in Figure 5.12. All graphs consist of the average cross-correlation functions of $46 D$. $d$. cells that were labeled with Raf1RBD-GFP and LimE-mRFP, as described earlier. In general, all cross-correlation 
maps look very similar. We find the strongest correlation coefficients without spatial shift and without temporal shift.

Regarding the dependencies of the two axes, it is clear that the spatial shift has a much stronger effect than the temporal shift. Even for small spatial shifts to either direction the correlation coefficients drops to or even slightly below zero. That means that the signals are not correlated, if the spatial shifts At fixed position for no spatial shift, the correlation also decreases strongly for temporal shifts. We find a small shift in the asymmetry plot of Figure 5.12 towards the negative time for the mean cross-correlation functions of curvature and Ras as well as for curvature and LimE. This anisotropy is maximal without a spatial shift and is on the order of $10 \%$ of the cross-correlation coefficients. In the case of the cross-correlation of Ras and LimE, we find the correlation function is again peaked sharply at no-shift position. Nevertheless, the anisotropy graph is quite different. At first the anisotropy is maximal at a shift of half a cell perimeter. In addition the anisotropy is smaller compared to the higher correlation functions and finally at no spatial shift position the anisotropy is slightly negative, corresponding to a potential mismatch of the timing of Ras and LimE, where LimE lags behind Ras.

The fact of the relatively high values at no spatial shift may be explained with the fact that we observe rather few pseudopods in the data. As due to imaging reasons, we observe any cell maximally for about one minute. On this time-scale, many cells have a conserved cell shape. Additionally, we plot here the cross-correlation only with temporal shifts of $-5 \mathrm{~s}$ to $5 \mathrm{~s}$ as we could include all 46 cells in this analysis. This can also be seen in the amount of pseudopods we show in the next section. 


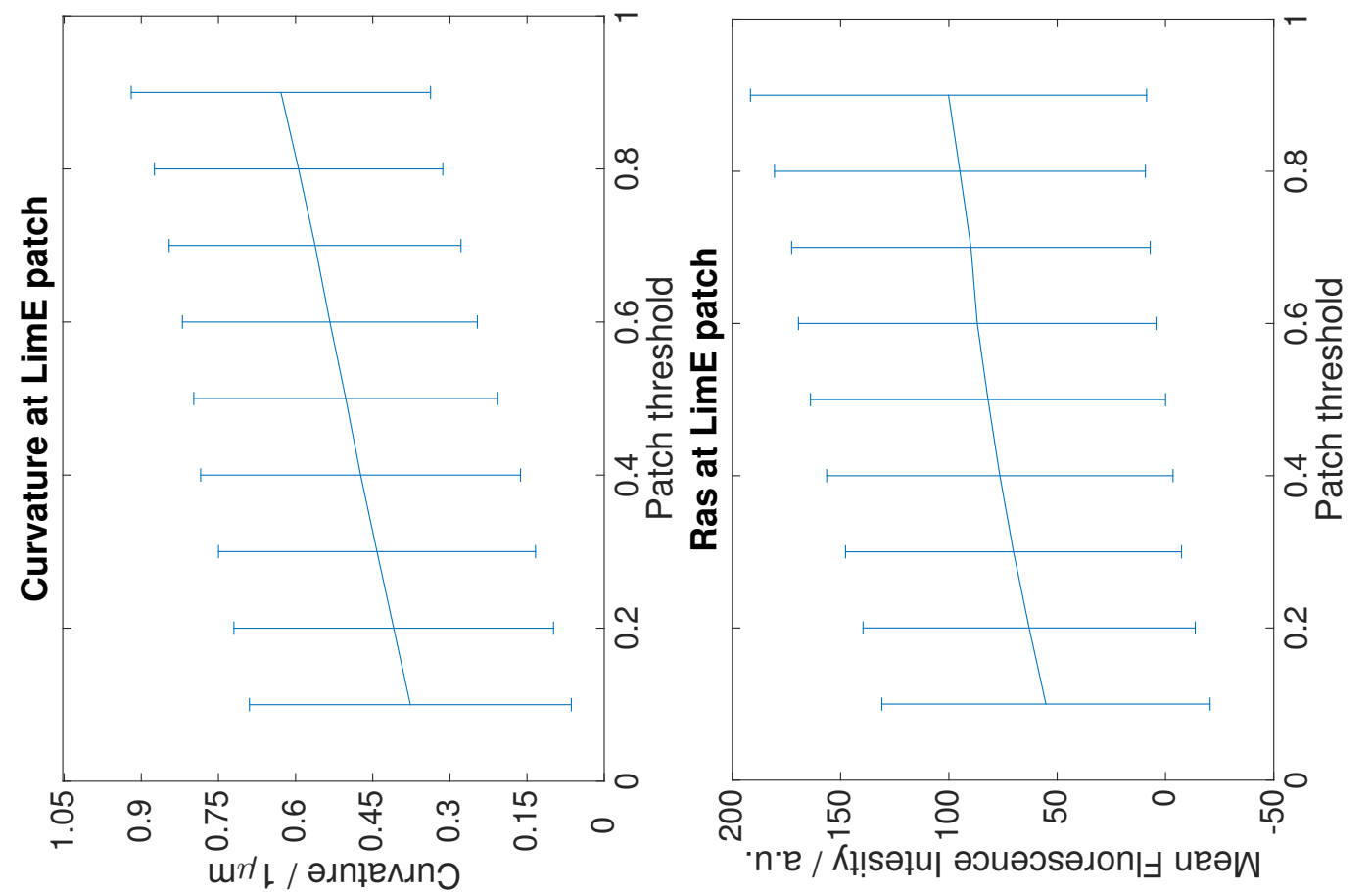

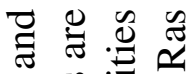

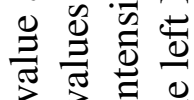

$\approx .0 \pm$

Ф્

\& $\dot{0} 0$

응

$\cong$ जि

完至

둔

可

늘 芒

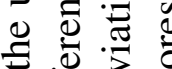

$\Xi$ 过

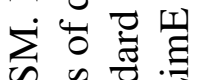

थ के च

च

क त.

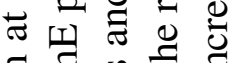

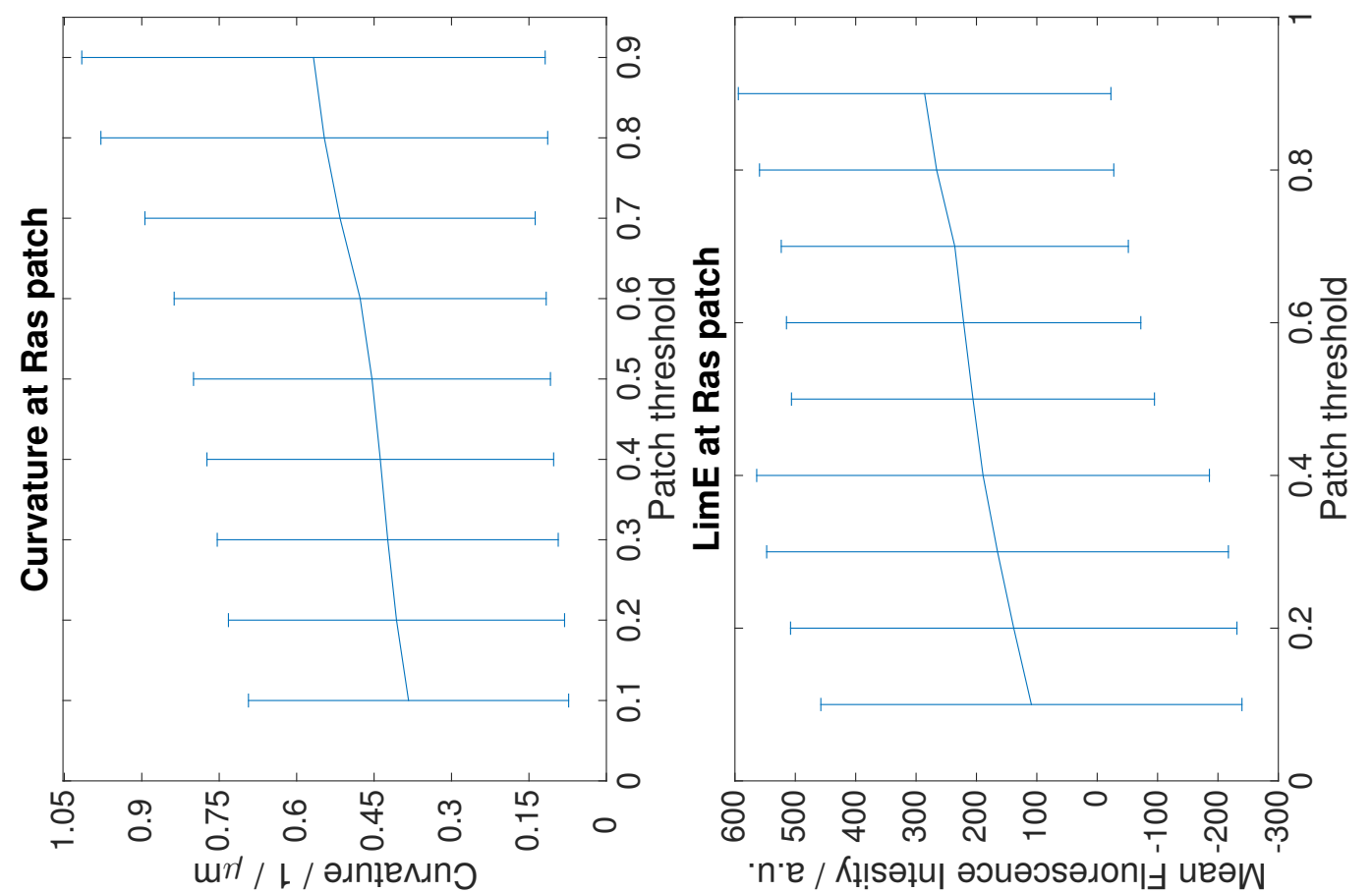

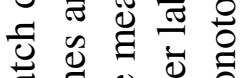
芩

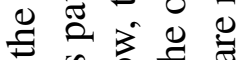

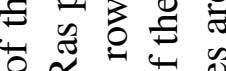
둥 \% 零 记 ญ \& $\Xi \Xi$ I ธี ๒ $\cong ี$ ㅎํ월

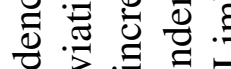

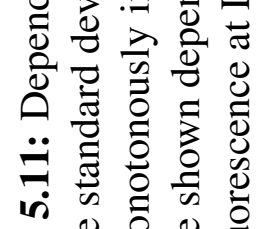
๖ 巳

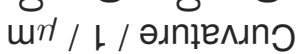

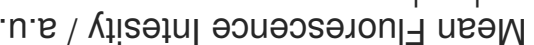




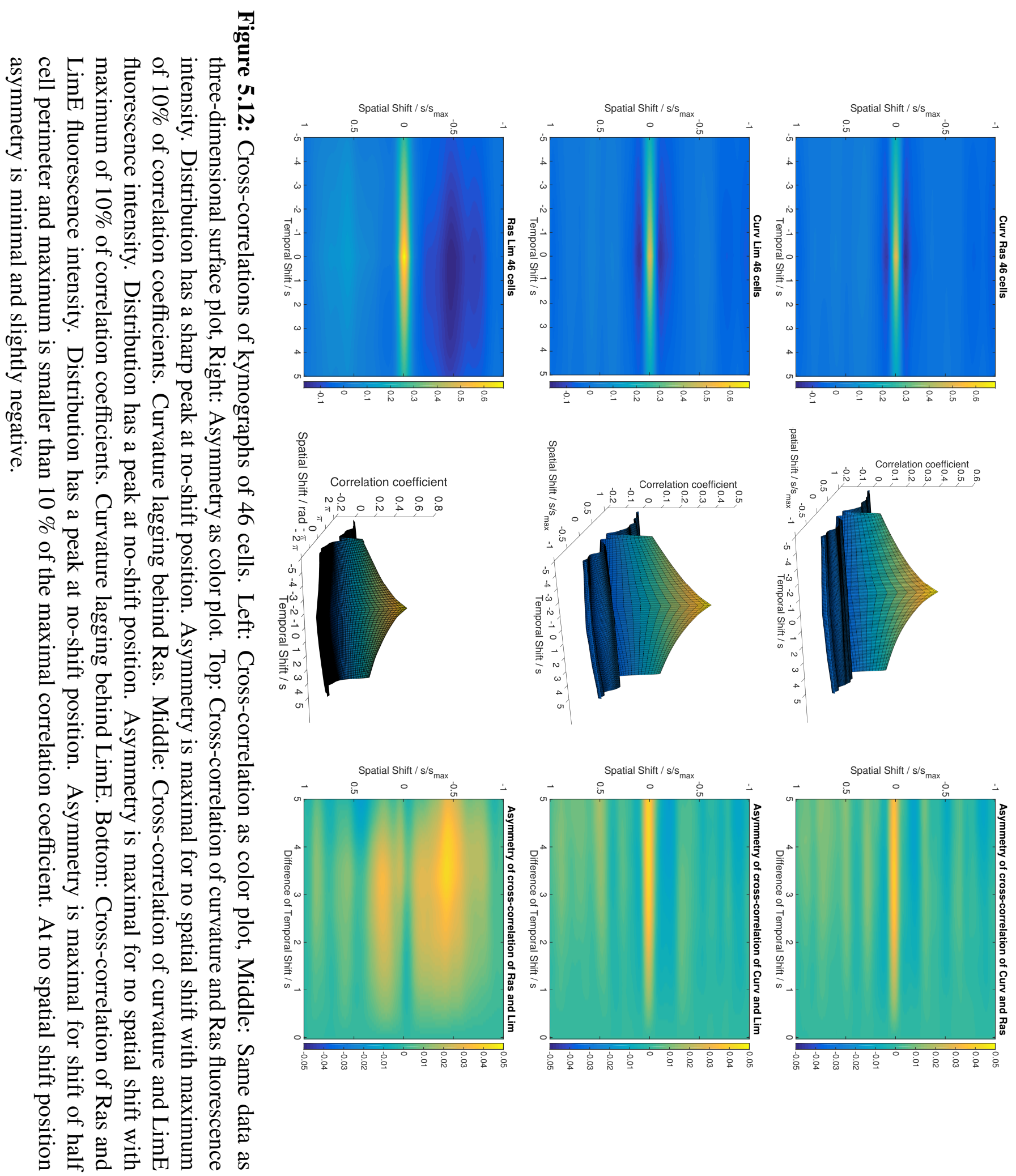




\subsubsection{Cross-Correlations of Pseudopod-Kymographs}

The analysis of the pseudopod splits is done exactly as for the case of the conventional CLSM. A general problem of the fast imaging, we can achieve with the sdCLSM is that the due to the high intensities of the excitation laser that is illuminating the whole cells all the imaging time. Hence, the cells were behaving reasonably only up to 2000 frames, corresponding, at a frame rate of 16.8979 frames/s, to less than 2 minutes. After that they show severe damage. on the one hand there is a strong reduction of fluorophores caused by photobleaching, on the other hand also the cells stop migrating and sometimes even release from the substrate. This can also be observed for conventional CLSM, but usually only for imaging times of the cells above 10 minutes. Therefore it was much more challenging to gain data for splitting pseudopodia. This is the reason why from 46 cells that could be investigated, we could extract only 33 pseudopodia, compared to the case of the conventional CLSM, where we extracted 121 pseudopodia from the data of 31 cells.

Via the two-dimensional cross-correlation of the curvature, Ras and LimE kymographs, we nevertheless could calculate the average cross-correlation functions of those 33 pseudopodia. The results are qualitatively very similar to those of the conventional CLSM in Figure 5.9. We can again find the maximal peak of the correlations at the exact no-shift position in all three cases. In the case of the correlation of curvature with one of the fluorescent labels an asymmetry with higher correlation to the negative times is visible especially in the asymmetry graph and its maximum has a value of about 0.8 , hence more than $10 \%$ of the original correlation coefficients. This is consistent with Figure 5.9 and with the literature. The small spatial dependencies, as seen above, are again visible but not as pronounced as before. The 
correlation of the two fluorescent labels does not show a strong asymmetry, but at no-shift position and for a time difference smaller than $2 \mathrm{~s}$ we can see the minimum of the asymmetry at -0.04 . This corresponds to a $7 \%$ negative asymmetry that indicates that the LimE signal is lagging against the Ras signal. 

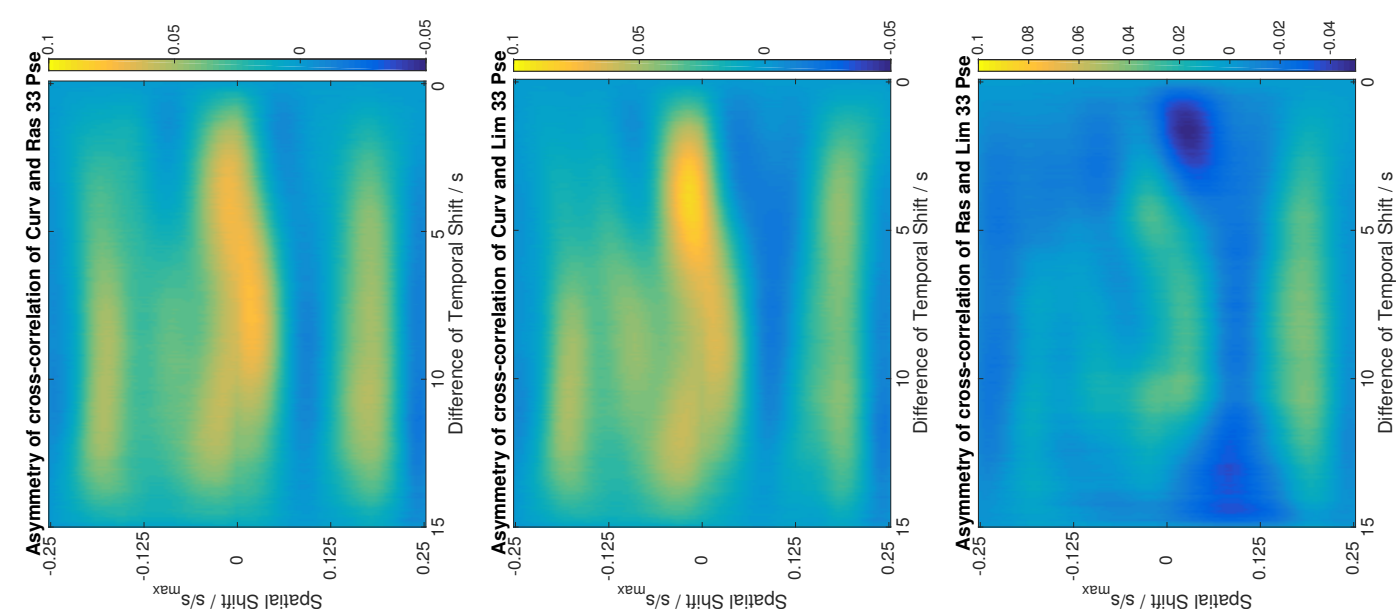

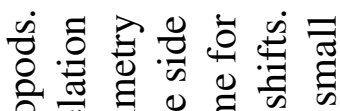

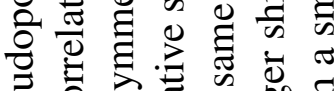

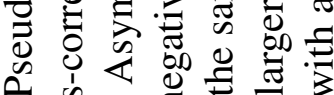

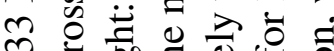

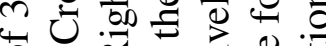

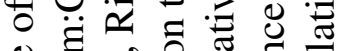
过 ए . 당

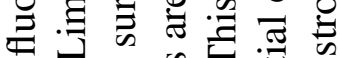
प्र 娄 可言 . ส

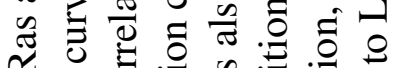
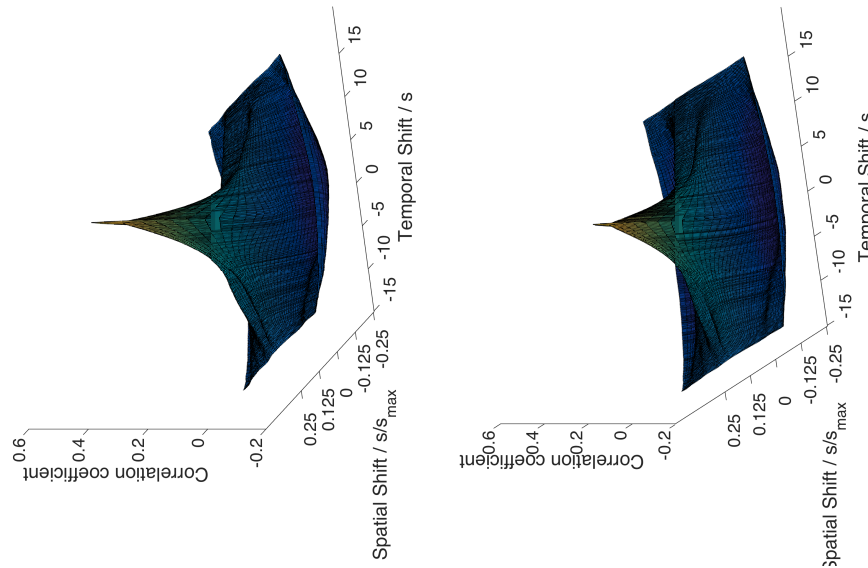

$\simeq$

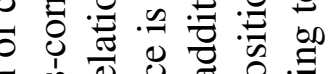

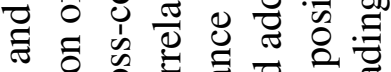

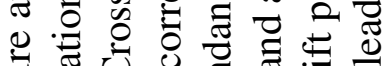
盯U U U ¿ $\exists$ J ญ

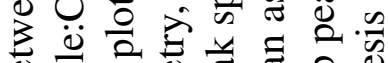

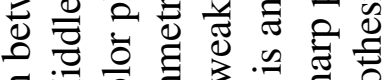

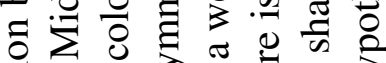
ن

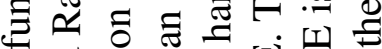

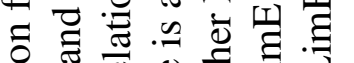

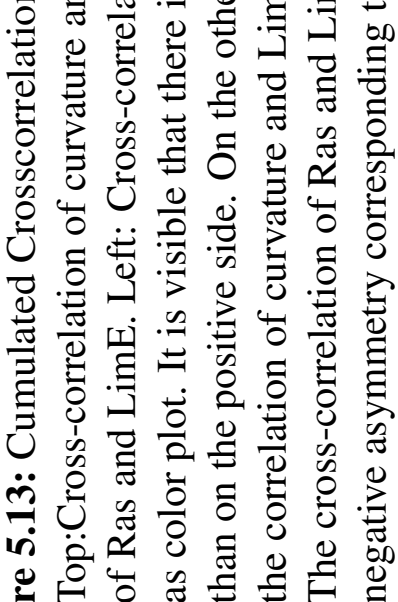
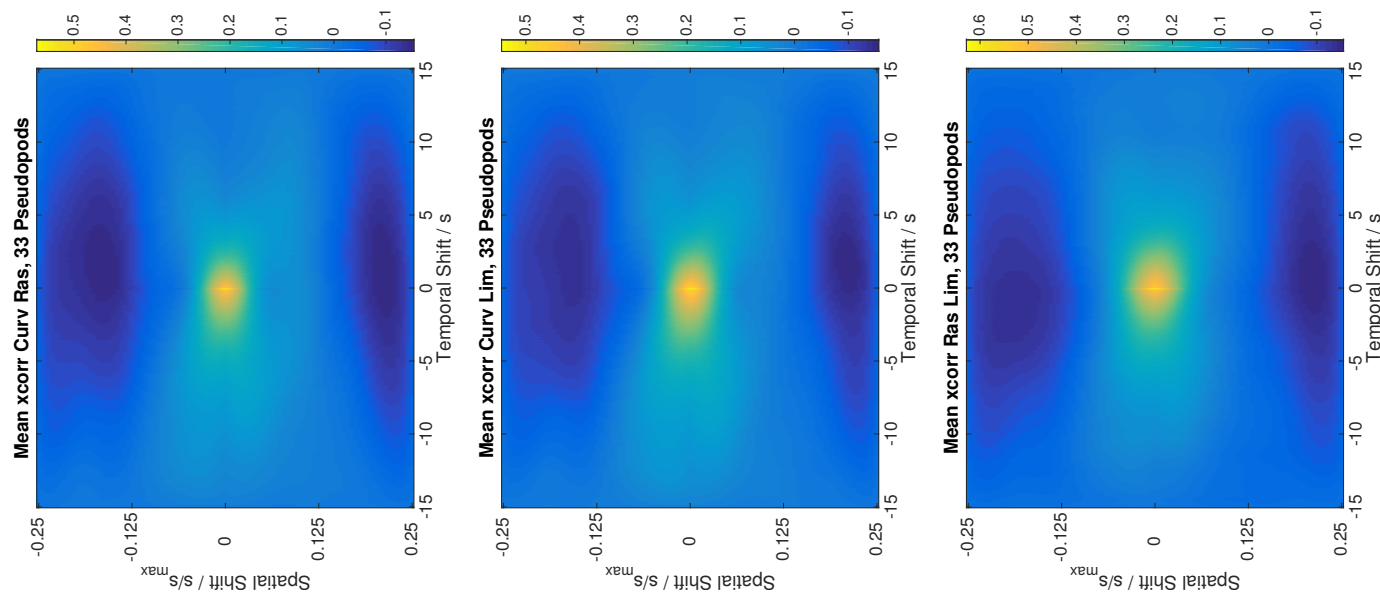

包 


\subsubsection{PTEN Knockout Cells}

To investigate the influence of the signaling cascade to the correlation between cell shape and cell signaling dynamics, we performed experiments with $D . d$. cells in which PTEN was knocked out. As described earlier in subsection 2.3.1, PTEN is a regulator of the reaction of PIP2 to PIP3. Hence it has a strong influence on the cell geometry and especially its symmetry breaking.

\subsubsection{Dependence on Fluorescence Patches}

As before for wild type cells, we also analyze the dependencies of the distributions of the lognormal fits of curvature and fluorescence intensities on the fluorescent patches for the knockout mutants where PTEN is disabled. The setting is exactly the same as before in subsection 5.3.1.1, besides the different cell line. We investigated 11 cells on three experimental days.

The curves on Figure 5.14 are all monotonously increasing for increasing value of the patch threshold. This holds for the curvature distributions for both conditions (Ras patch or LimE patch) as well as for the distributions of the fluorescence intensities conditioned of the other fluorescent patch. For all curves but LimE at Ras patches, the mean values start to saturate at a threshold of 0.7 . In contrast, the mean value of the LimE distribution at Ras patches is increasing in steepness at a threshold of 0.7 .

In addition we realize that the standard deviations of the fitted curves are increasing with increasing patch threshold. This may be due to the smaller number of cells and especially due to the strongly reduced number of pseudopod splits (one) because of the measuring technique and cell line. The PTEN-null cells generally show less polarization and hence fewer splitting pseudopods than wild type cell lines. 


\subsubsection{Cross-Correlations of Kymographs}

The results obtained with the mutant cells are shown in Figure 5.15. The data analysis is the same as described before for the wild type cells. Also the results are very similar to those of wild type cells shown in Figure 5.13. We find relatively high correlation for all temporal shifts when the spatial shift is close to zero. In all cases the maximal correlation coefficient is around 0.7 . In addition there is no asymmetry visible on neither of the asymmetry maps. The reason for these findings is the same as for the wild type cells. There are only very few shape changes occurring. Within the 29 kymographs gathered from 11 cells, we could only find a single pseudopod split and the stable cell curvature and hence kymographs with horizontal stripes are leading to this strong dependency of the spatial shift.

For the experiments with the PTEN-null cells we could not find any temporal asymmetry in neither of the cross-correlations. This may be explained by the fact, that there is only very few active migration for this cells. These cells are known to be less motile, form less pseudopods and show less splitting of pseudopod. Additionally the analysis to extract the cell outline is more difficult, as the cell outline is less regular for this cell type.

\subsubsection{Cross-Correlations at Pseudopod Level}

The analysis of the pseudopod splits is done exactly as for the wild type cells. As explained above, the PTEN-null cells tend to form fewer pseudopods as the wild type cells. As the sdCLSM only allows for small imaging times, the probability to find a pseudopod splitting is significantly reduced.

From 11 PTEN-null cells measured on three experimental days we could only 
extract a single split of a pseudopod. Via the two-dimensional cross-correlation of the curvature, Ras and LimE kymographs we nevertheless could calculate the cross-correlation functions of this pseudopod. The results are clearly showing that for a single pseudopod, the cross-correlation functions do not have this high values for all values close to a spatial shift of zero but a much more distinct peak. In addition, we see that the spatial axis possesses an asymmetry, which is due to the asymmetric splitting process, where one of the two pseudopods survives and the other one dies out. Still, we find the maximal peak of the correlations at the exact no-shift position in all three cases. But here we find a strong anti-correlation at a negative spatial shift between $-0.25 s_{\max }$ and $-0.125 s_{\max }$. In contrast to the earlier presented data, the temporal asymmetry plays is rather negligible. 


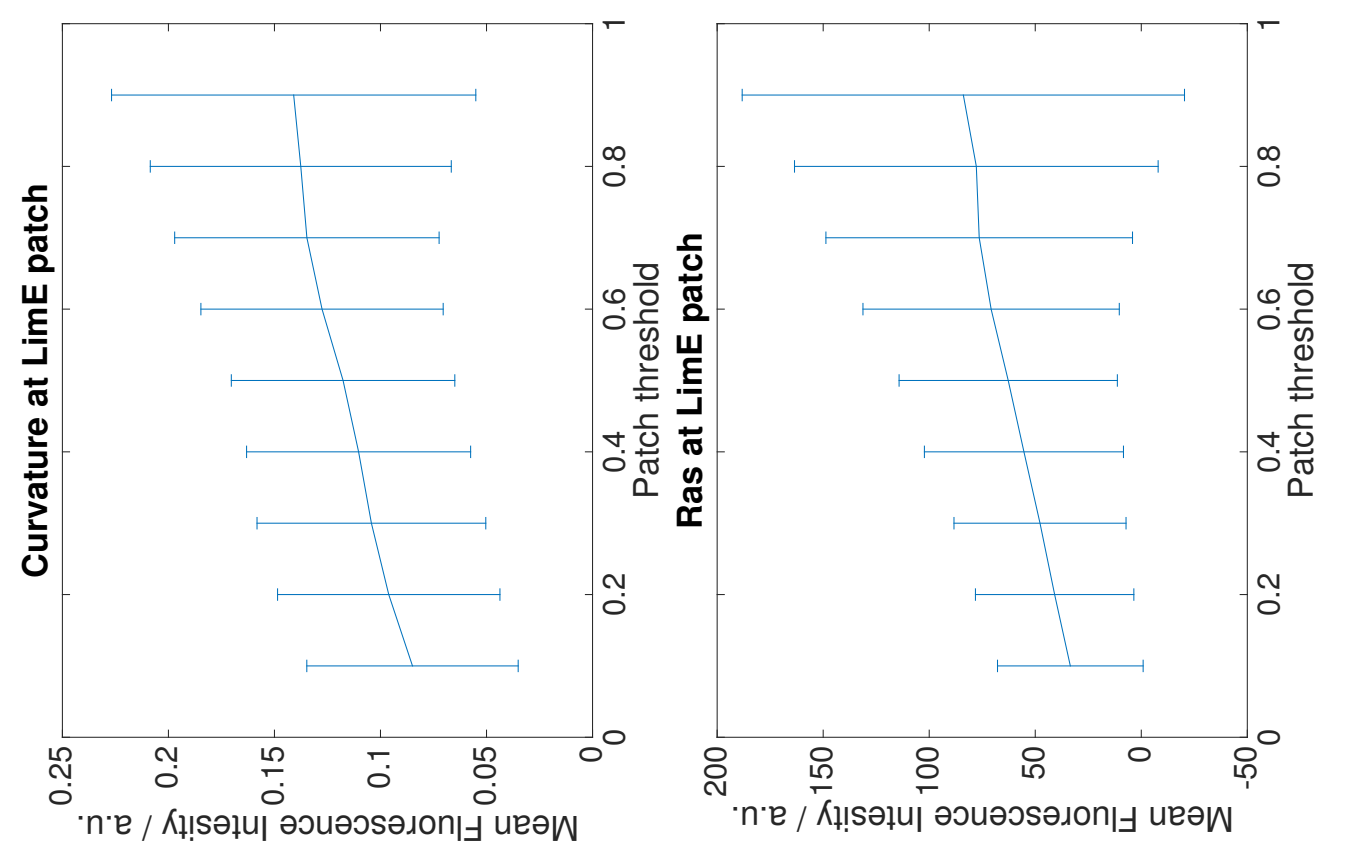

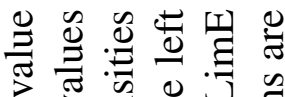
₹ 0 过

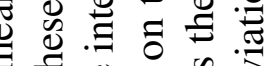
\& 응

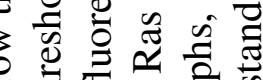

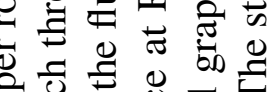

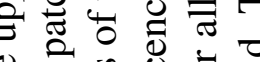
\&

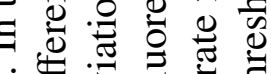

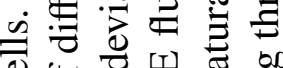

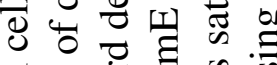

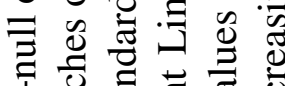
$\sum_{\mathrm{W}} \mathrm{w}_{\mathrm{W}}$

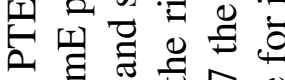
t)

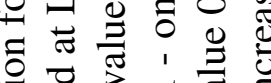

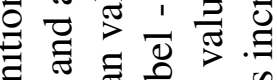
卷

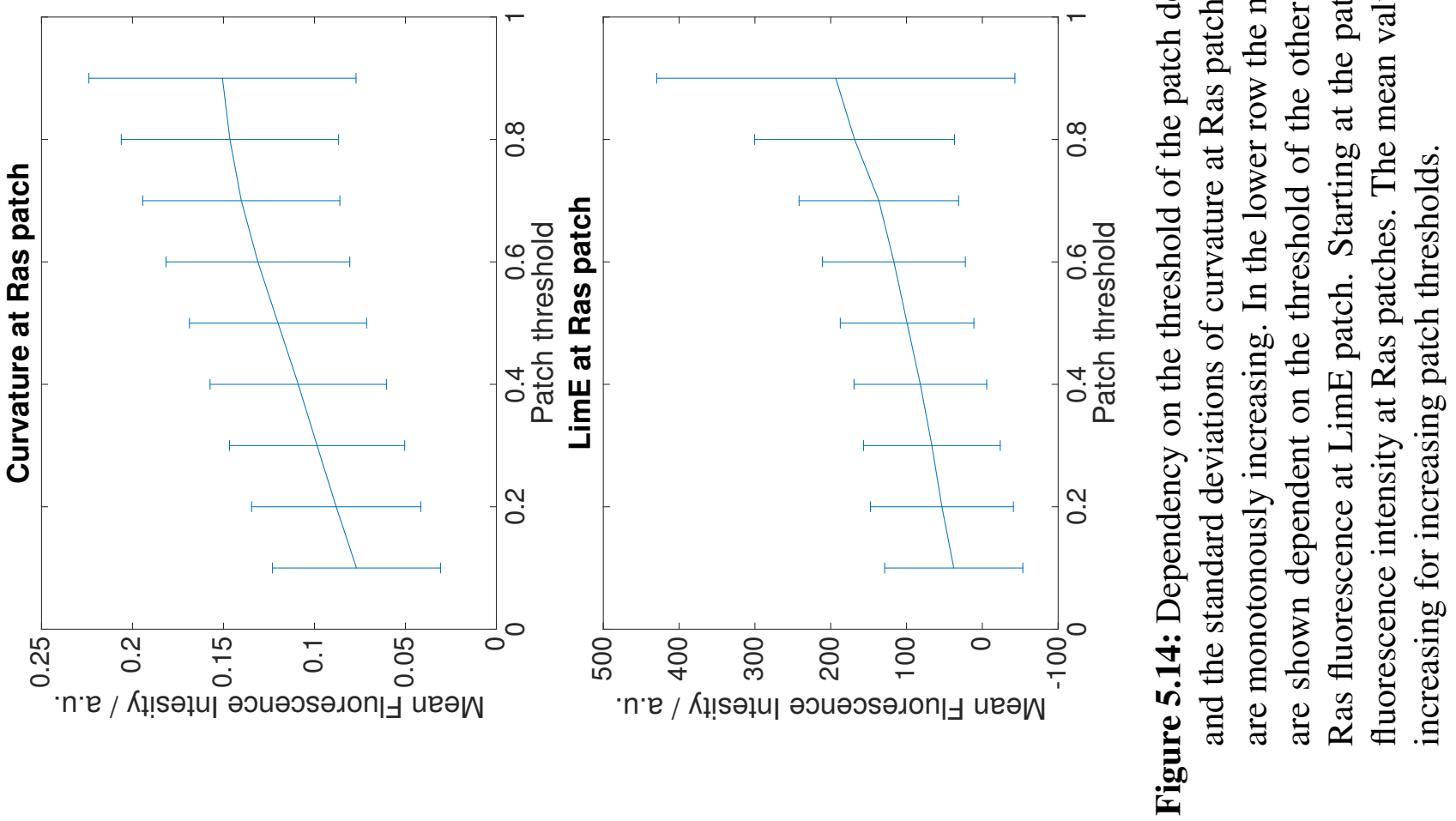



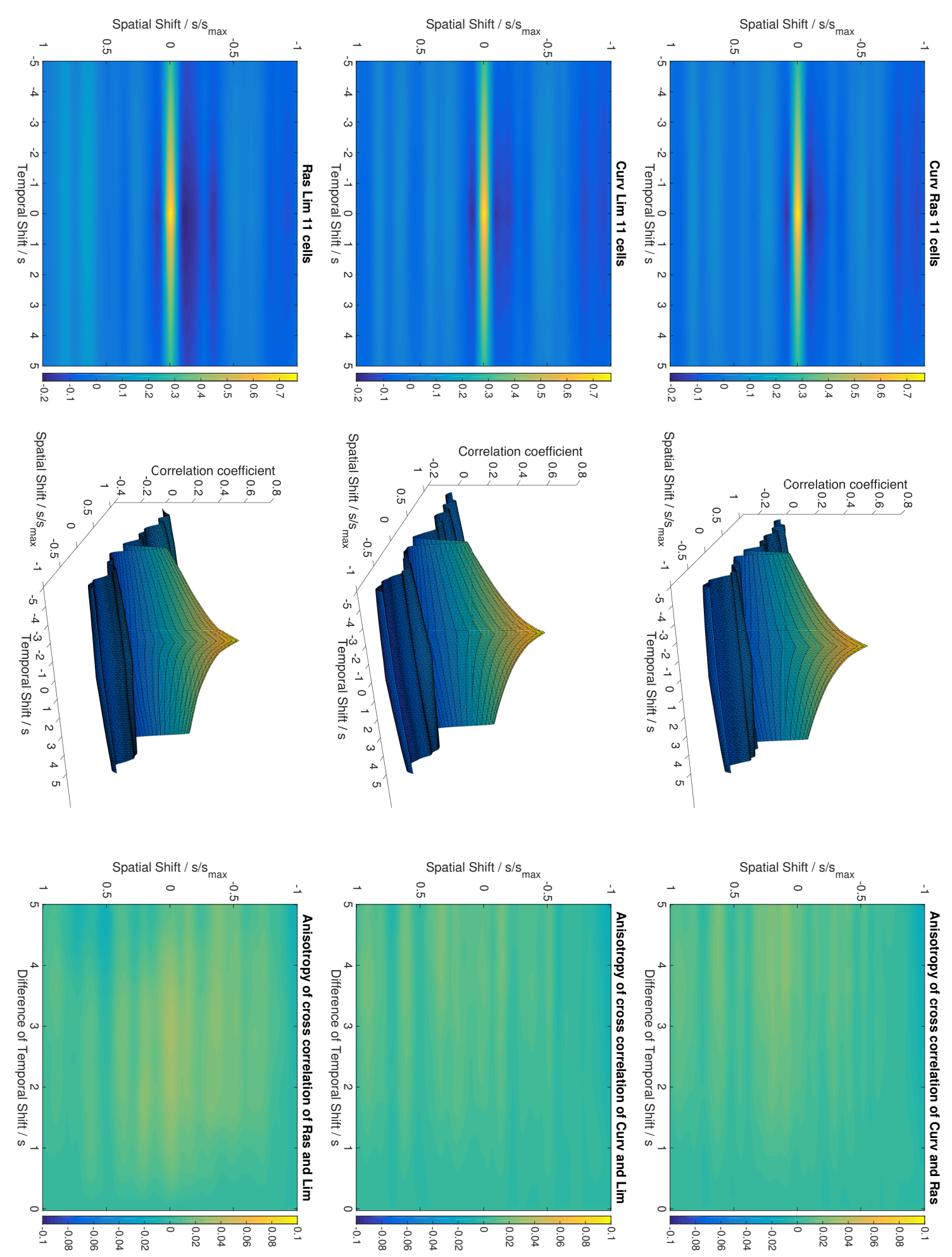

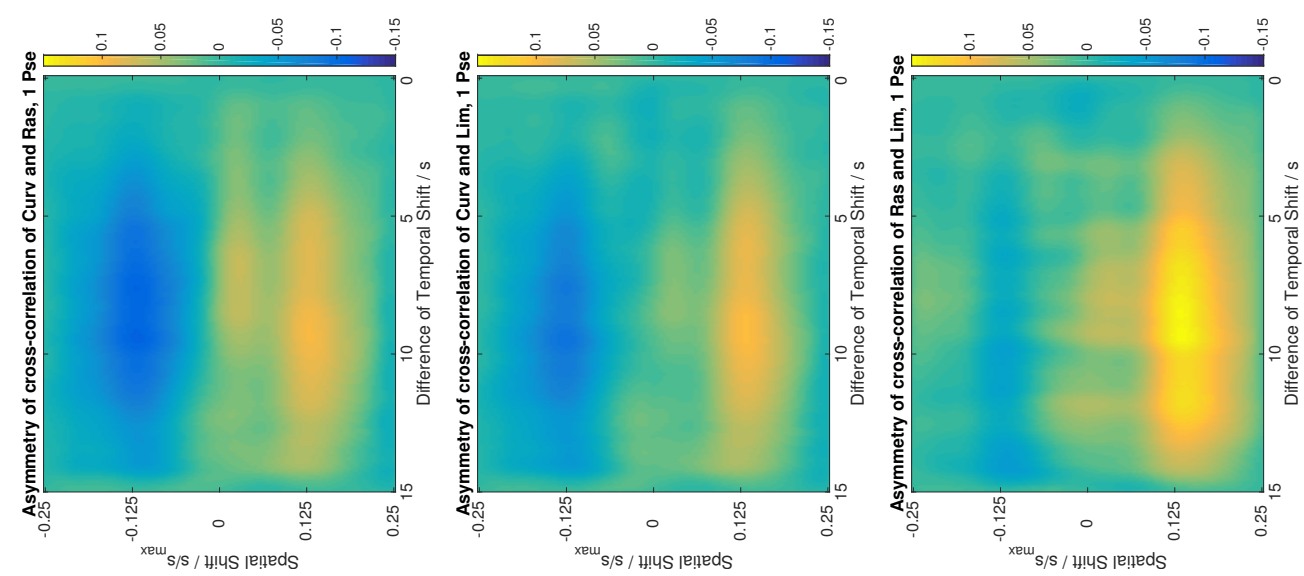

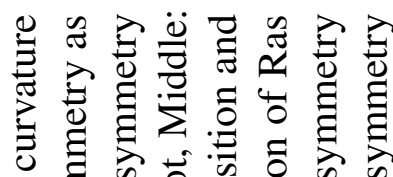

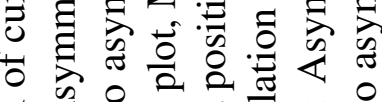

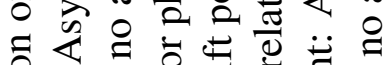

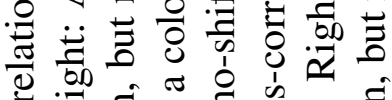

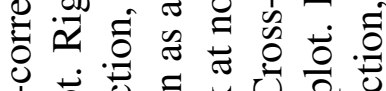
iे

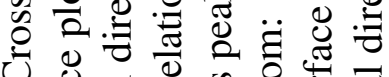
U. के $\dot{\Delta}$ के च ल

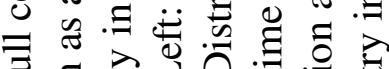

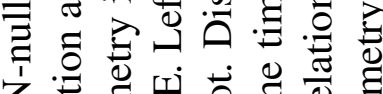
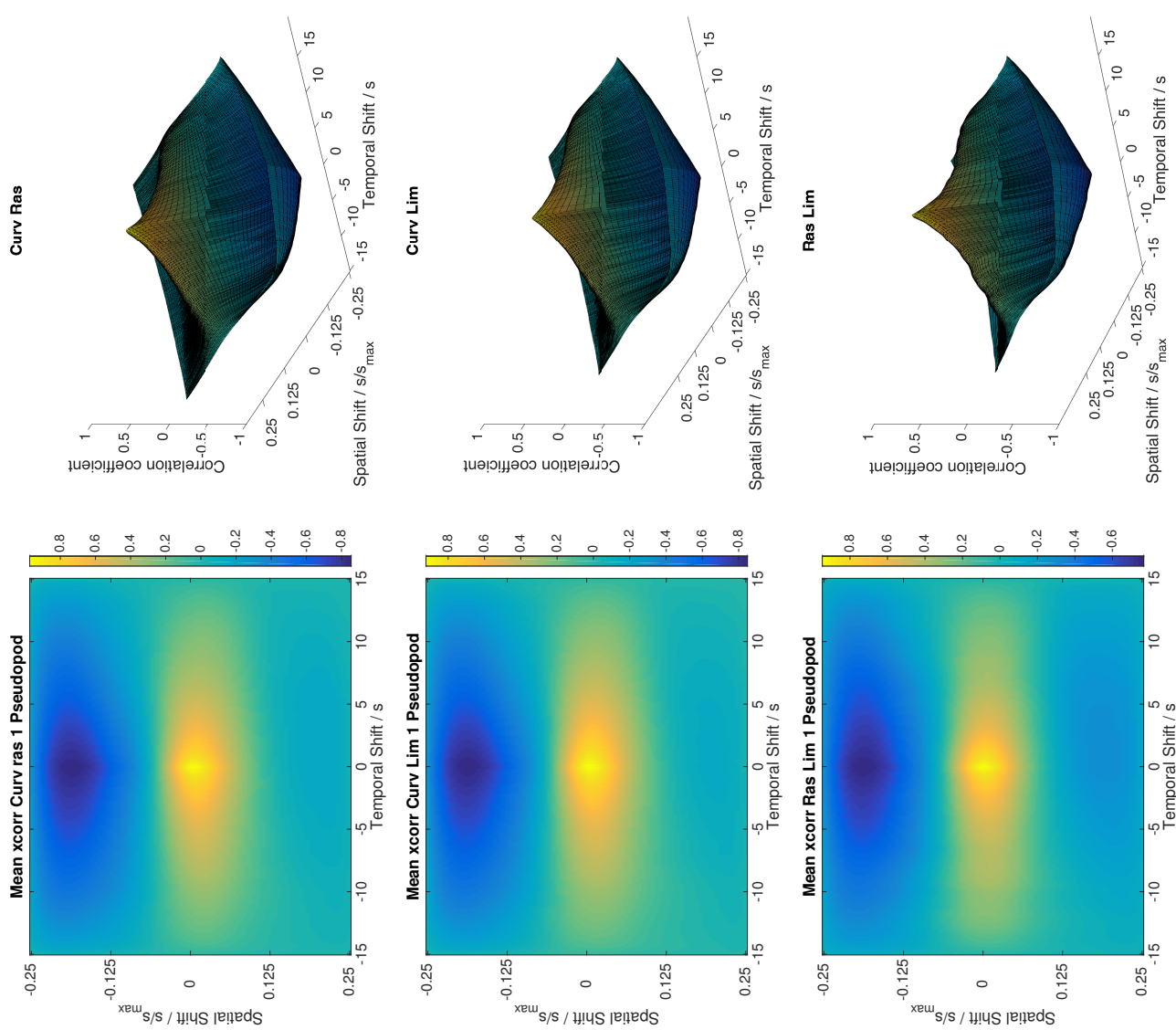

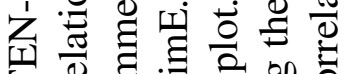
क 二 仓 छ ह

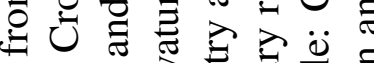
导导

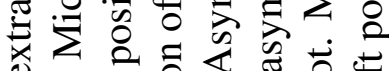
ரे

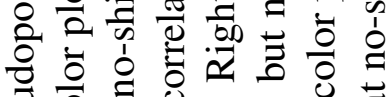
응

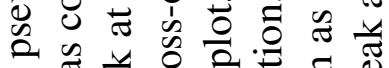

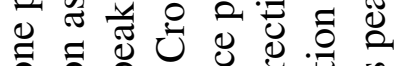
б.

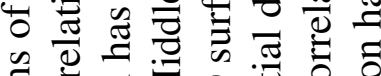

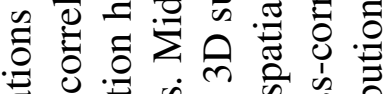

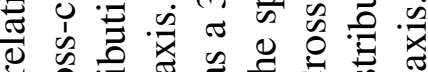

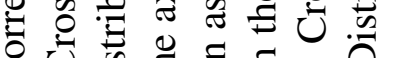

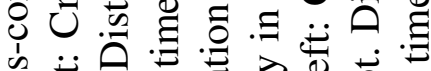

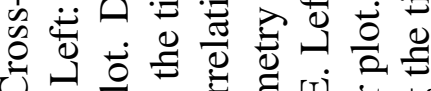

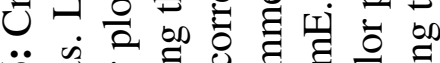

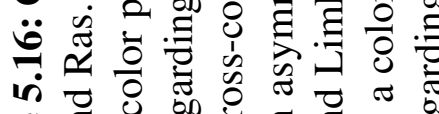
i⿱ $)$

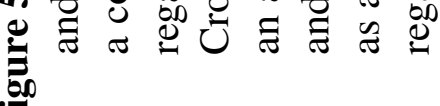




\subsubsection{Myosin-II Knockout Cells}

We performed further experiments with $D$. $d$. cells where myosin-II was knocked out to investigate the influence of the cytoskeleton on the spatiotemporal behavior of cell shape and Ras-G activity. Cells with weakened cytoskeletal contractility may show different behavior and may lead to a deeper understanding of the process. Hence we use the same experimental setup (microfluidic gradient mixer, concentration gradient $0.4 \frac{\mathrm{nM}}{\mu \mathrm{m}}$, sdCLSM) to investigate $16 D$. $d$. cells with a knockout of the myosin-II heavy chain that are labeled with the same fluorescent markers as before (Raf1-RBD-GFP+LimE-mRFP).

Due to the few available data the histograms of the distributions possess to few points and hence there is no reasonable fit possible. Therefore we do not show this data for the Myosin-II knockout cells.

\subsubsection{Cross-Correlations of kymographs}

In Figure 5.17 we show the cross-correlation results of 24 kymographs extracted from 16 different cells. Compared to the results shown in Figures 5.13 and 5.15, the myosin-II-null data is very similar to those results not showing a big difference. Similarly, the amount of extracted pseudopod splits that could be extracted is very small, namely three.

There is no significant asymmetry in the plotted cross-correlations. The crosscorrelation are rather high, without showing a hint to answer our question of the connection of Ras-G activity, actin polymerization and cell geometry. We can not tell temporal or spatial shifts between any two of the three signals. This can be explained by the few events of splitting pseudopods, the small amount of cells that could be 

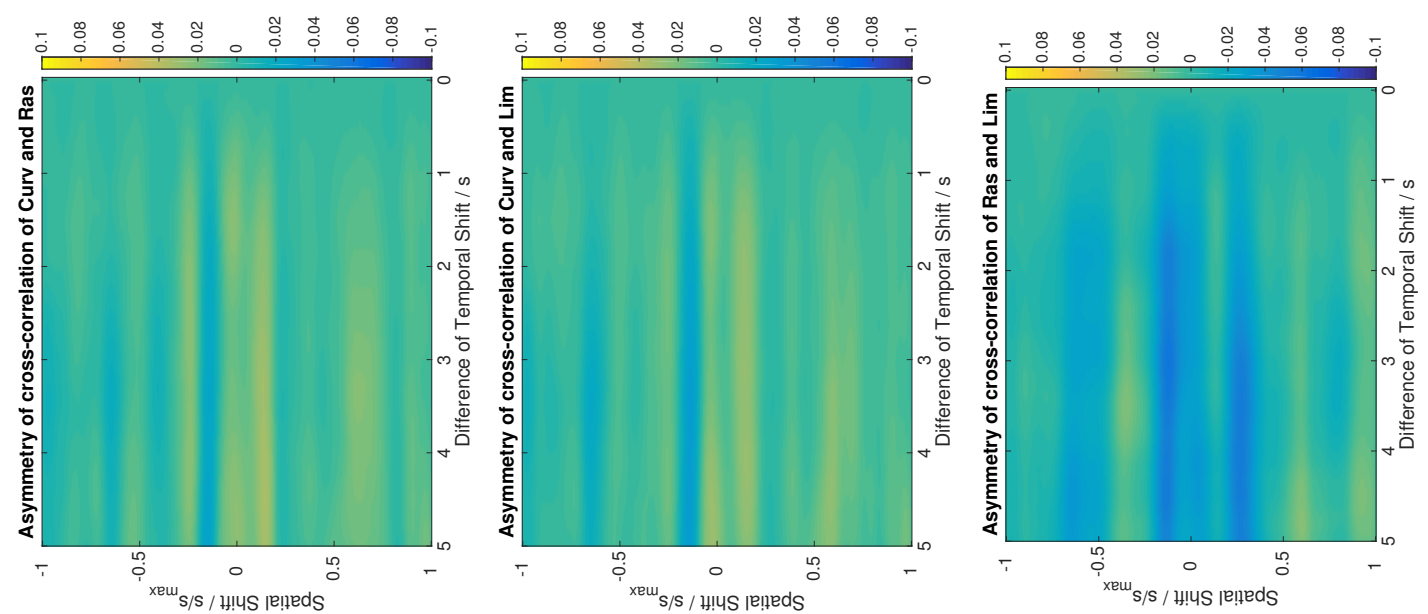

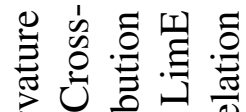

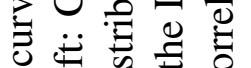
엉

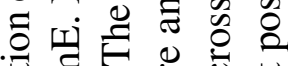
䒕 밈 을 可

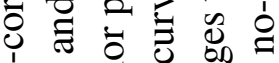
क ज 은 फั0

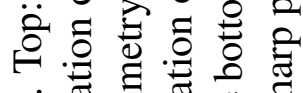
$\dot{0} \frac{\pi}{0}$ 过

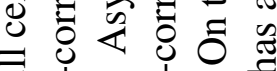
寻
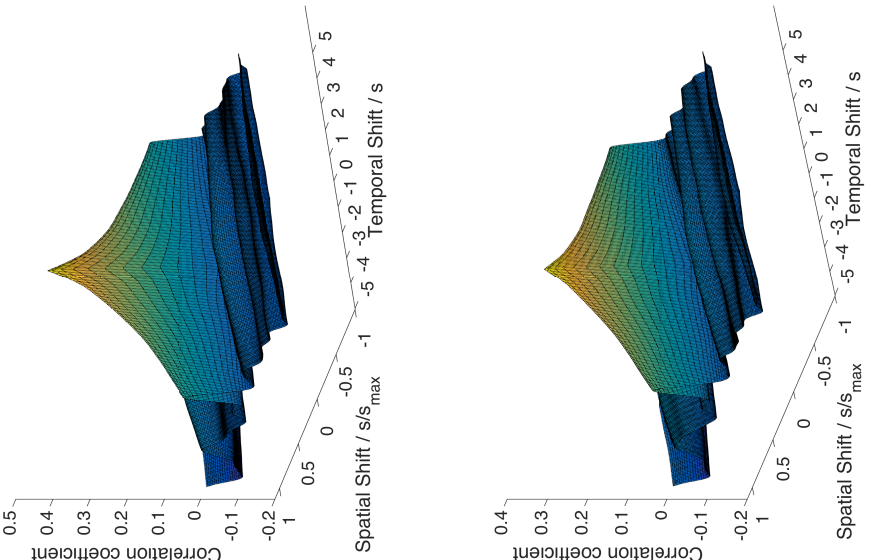

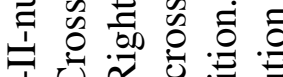
IUน U

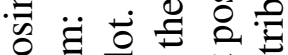

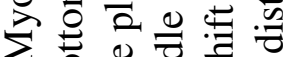

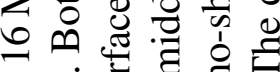
छ山嵌 ○ี

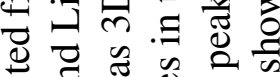

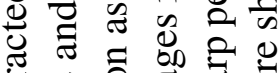

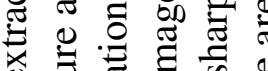

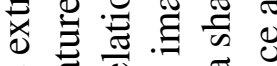

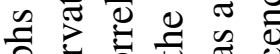

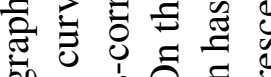

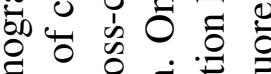
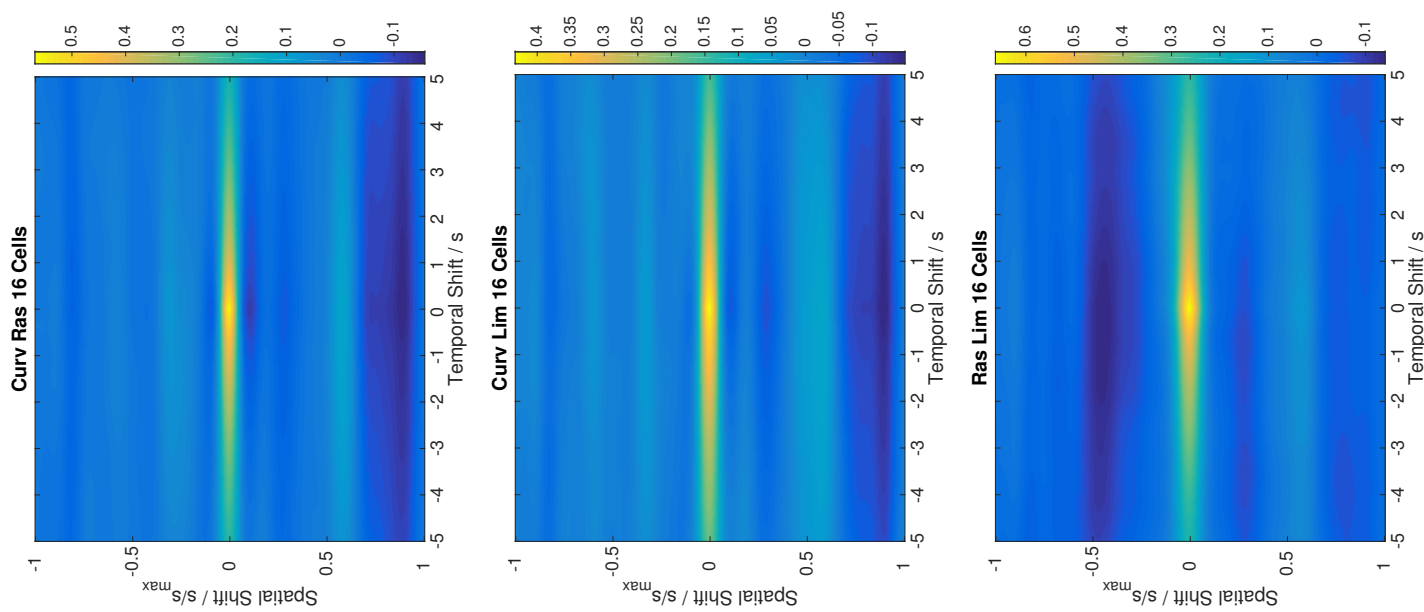
ह 응 흘 추음음 ป

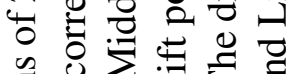
๘ 咅

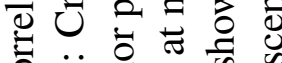

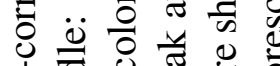
点 ○ं

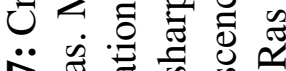

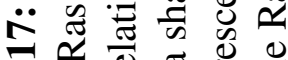
in 平

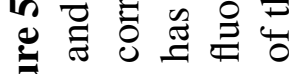
党 
analyzed and the big differences of the cell shape of the cells due to their genetical modification. As mentioned earlier the imaging with the sdCLSM has generally the problem of short imaging time and hence fewer splitting events. Also the amount of cells that showed the fluorescent label in both Ras-G and LimE signals was smaller, assumably due to the cell type, as the defect of missing myosin-II heavy chain is a severe problem of the cells. This causes also that these cells are usually very big and hence show fewer efficiency in chemotactical migration. This may be caused by the lack of regulation of the pseudopod splitting, due to missing myosin-II contraction.

\subsubsection{Cross-Correlations at Pseudopod Level}

In Figure 5.18 we show the average cross-correlation maps of those three pseudopod splits of myosin-II-null cells. As the amount of pseudopod splits is so small we can not be sure, how much this data is dependent on cell-to-cell variability and how genreal these findings are. On these maps there are anisotropies visible. We find an asymmetry for all three cross-correlation maps. In the case of the correlations of curvature with Ras and LimE there is a asymmetry for negative times yielding again that the two fluorescent signals lead to the curvature signal. In the case of the correlation between Ras and LimE, we find an asymmetry for positive times corresponding to the fact that Ras would lag behind LimE. This would be a difference of the myosin-II knockout cells compared to the WT cells where we could find that LimE lags behind Ras. This may be explained with the fact of a faster reaction of actin polymerization without contractile forces of myosin-II. 

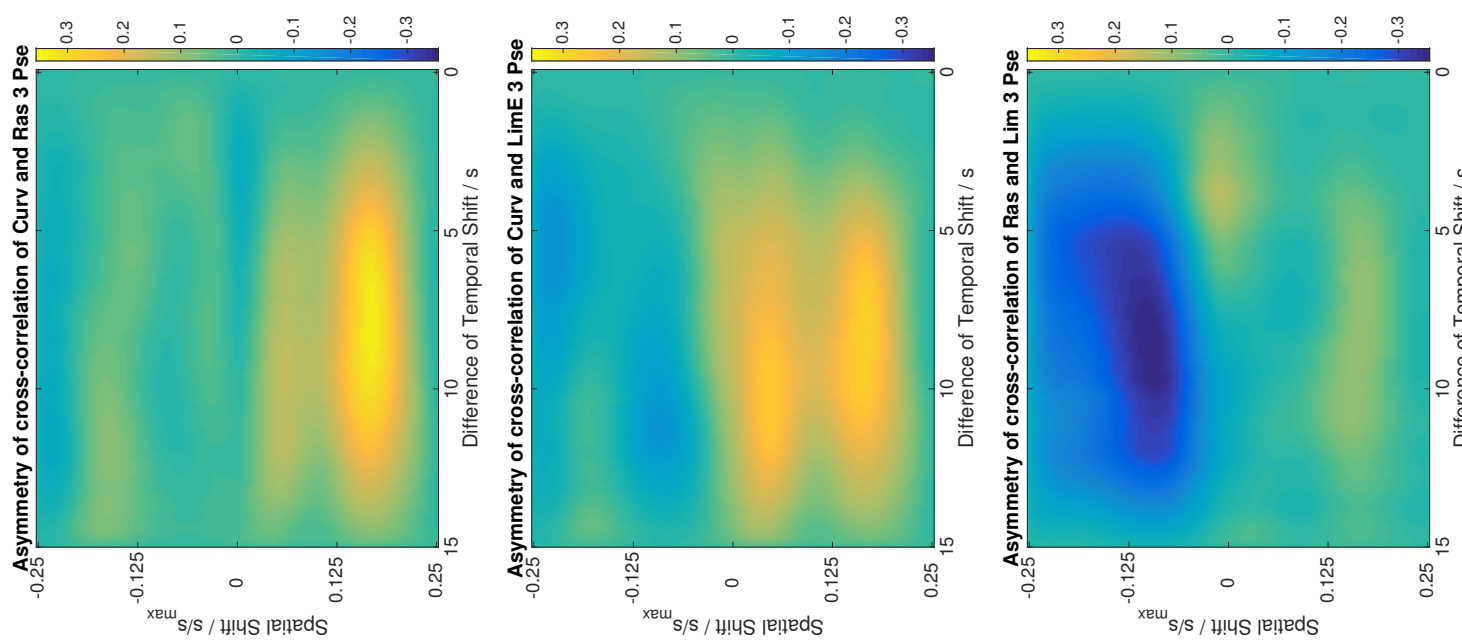

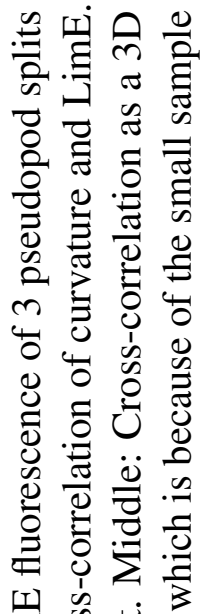

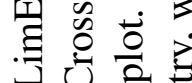

을 흥
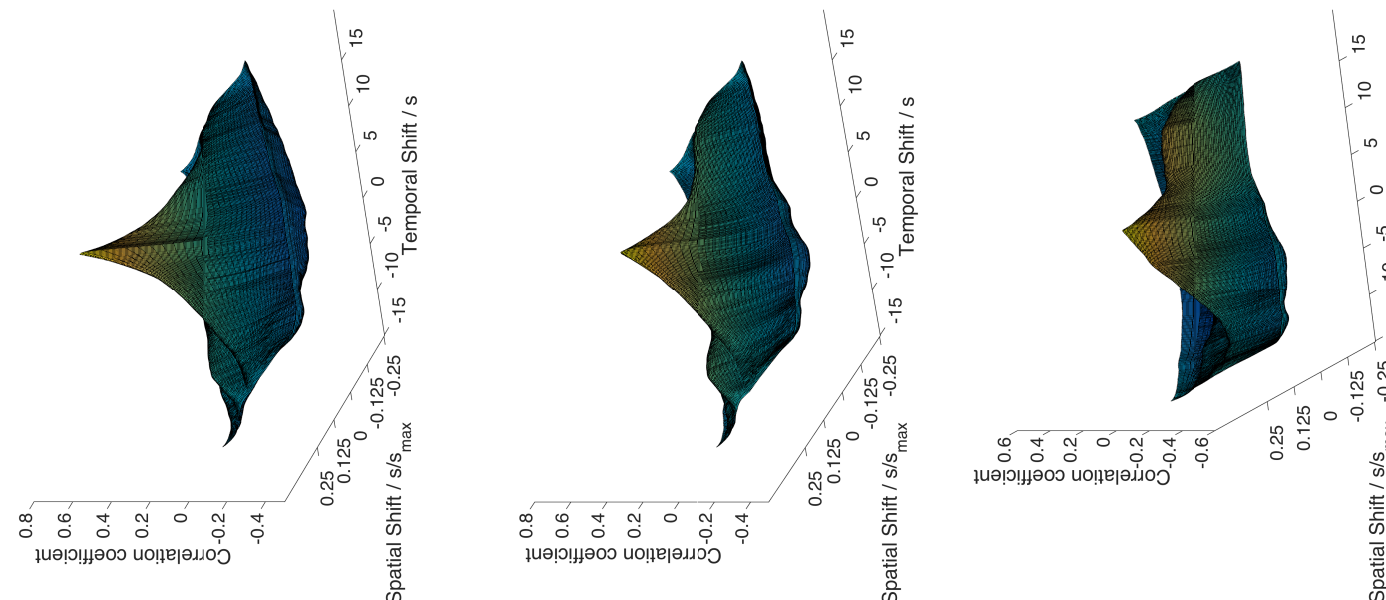

च 0 范

ส

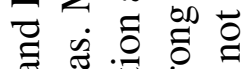

के

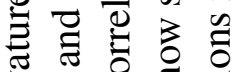

$\gtreqless$ 论

$\exists$

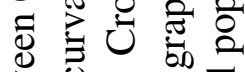

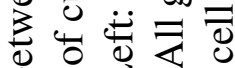

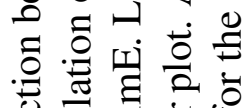

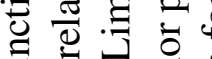

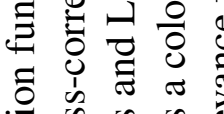
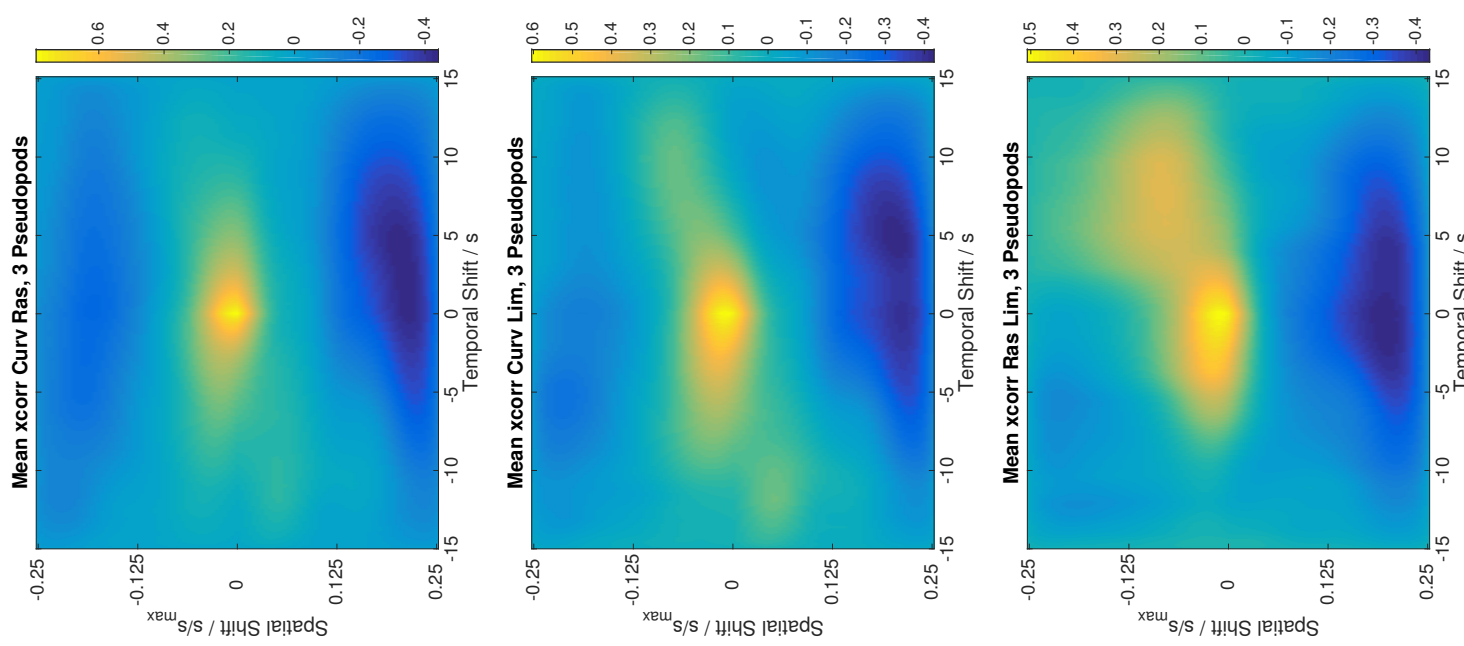

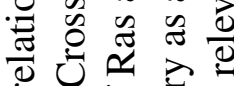

$\Rightarrow$ 능

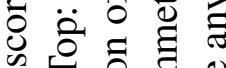

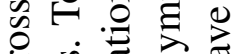

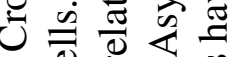

过

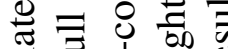

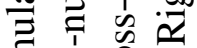

声声完苂

$\ddot{0} \ddot{\circ} \ddot{0}$

in

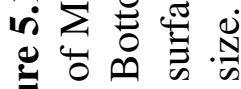

言范品言数 


\subsection{Discussion}

In this chapter, we investigated the spatiotemporal correlation of the cell shape, visualized by the membrane curvature, and the active biochemistry, visualized by fluorescent labels of the important signaling protein Ras-G and the cytoskeletal key player actin.

To find those correlations, we used double labeled $D . d$. cells inside a chemoattractant gradient, produced by a microfluidic gradient mixer. We were able to find a temporal asymmetry that showed us that the curvature lags behind the fluorescent signals. As the temporal resolution (2 frames/s) of the used conventional CLSM was not good enough to make more precise statements, we performed further experiments using a sdCLSM in cooperation with the group of Prof. Jörg Enderlein from the Third Institute of Physics of the University Göttingen.

Here, we reached a higher temporal resolutions (16.8979 frames/s) and, hence, more temporal details. Unfortunately, the imaging of around 1000 frames per minute led to the problem that the cells could not be observed longer than 1.5-2 minutes. Hence, the amount of single pseudopodia was lower than for the CLSM setup. For the data of single pseudopod splits, we could again find an asymmetry but for the whole cell level we could not.

We could proof that the curvature distribution at fluorescent patches is shifted to more positive values compared to all curvature values.

Regarding the splitting pseudopods, we find asymmetry of the curvature and the fluorescence in such a way that the curvature is lagging behind the fluorescent signals.

Due to the high temporal resolution data of the sdCLSM, I was able to find an 
asymmetry between Ras- $\mathrm{G}$ and actin, thus showing that actin is following Ras-G. These findings are shown in Figure 5.19 and the positions of these maxima can be found in Table 5.1. The maximal asymmetry for the cross-correlation of curvature and both fluorescent labels is very similar for each cell line (blue and yellow curves). I do not find a strong maximal asymmetry for Ras vs. LimE but for minimal asymmetry instead. Hence, we show that LimE is lagging behind Ras. On the level of splitting pseudopodia, the results are similar, besides the fact, that the values of the asymmetry are larger. As for the mutant cells, the amount of pseudopod data is small, so that it is not certain how significant the findings are. For PTEN-null cells, the values are comparable to the WT cells investigated by sdCLSM. Contrarily however the values for the Myosin-II-null cells are very high.

What we would need additionally is to increase the amount of pseudopod split data for the high temporal resolution and especially for the mutant data to able to make statements about the effects of the missing proteins.

Very interesting would be for further investigation to increase the analysis to three dimensions. Performing experiments with high temporal resolution in such a way that the fluorescence activity and the cell geometry could be analyzed in full three dimensions could help to give a more complete and volumetric image of the full process. 

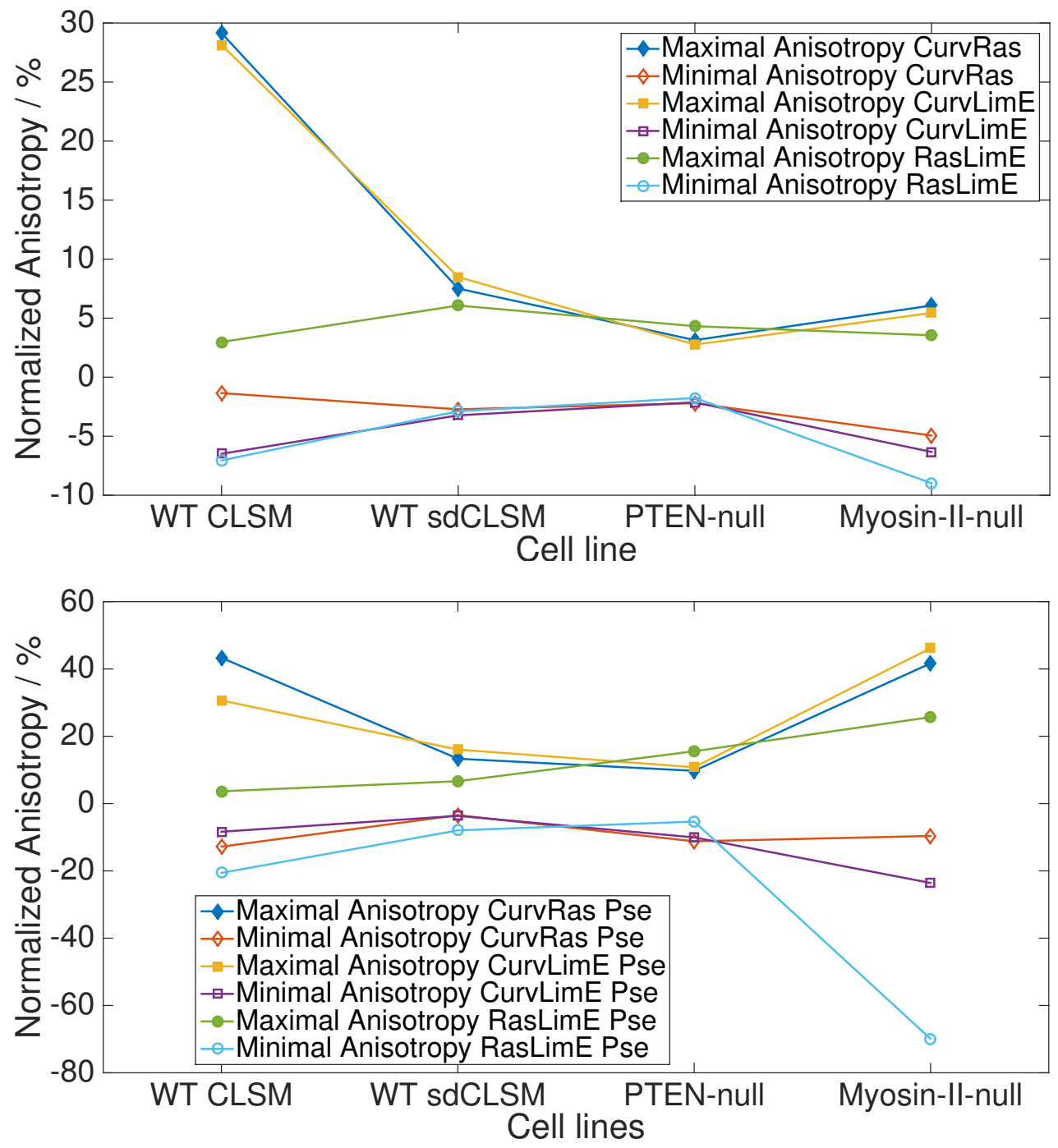

Figure 5.19: Overview of asymmetry of cross-correlation functions. Maximal asymmetry normalized to the maximum cross-correlation coefficients of the corresponding mean cross-correlation functions on the whole cell level. Filled symbols represent maxima while open symbols represent minima. Top: Analysis on whole cell level: Blue and yellow curves (CurvRas and CurvLimE) are in very good agreement. The smallest values for maxima between curvature and fluorescence intensity is found for PTEN-null cells, largest values for WT cells in conventional CLSM. The maximal asymmetry for RasLimE is quite small. The light blue and violet curve are in very good agreement. For WT CLSM cells, there is an asymmetry of more than 5\% for RasLimE and CurvLimE, while it smaller than $3 \%$ for CurvRas. As well the RasLimE data has an aisotropy for myosin-II-null cells of $8.98 \%$. Bottom: Analysis on level of splitting pseudopods: Compared to top graph the asymmetry is higher on the pseudopod level. The values for pseudopodia for the knockout mutants are gathered for 1(PTEN-null), respectively 3(Myosin-II-null), hence the significance of this data is not certain. The minimal asymmetry of RasLimE shows a strong asymmetry. 


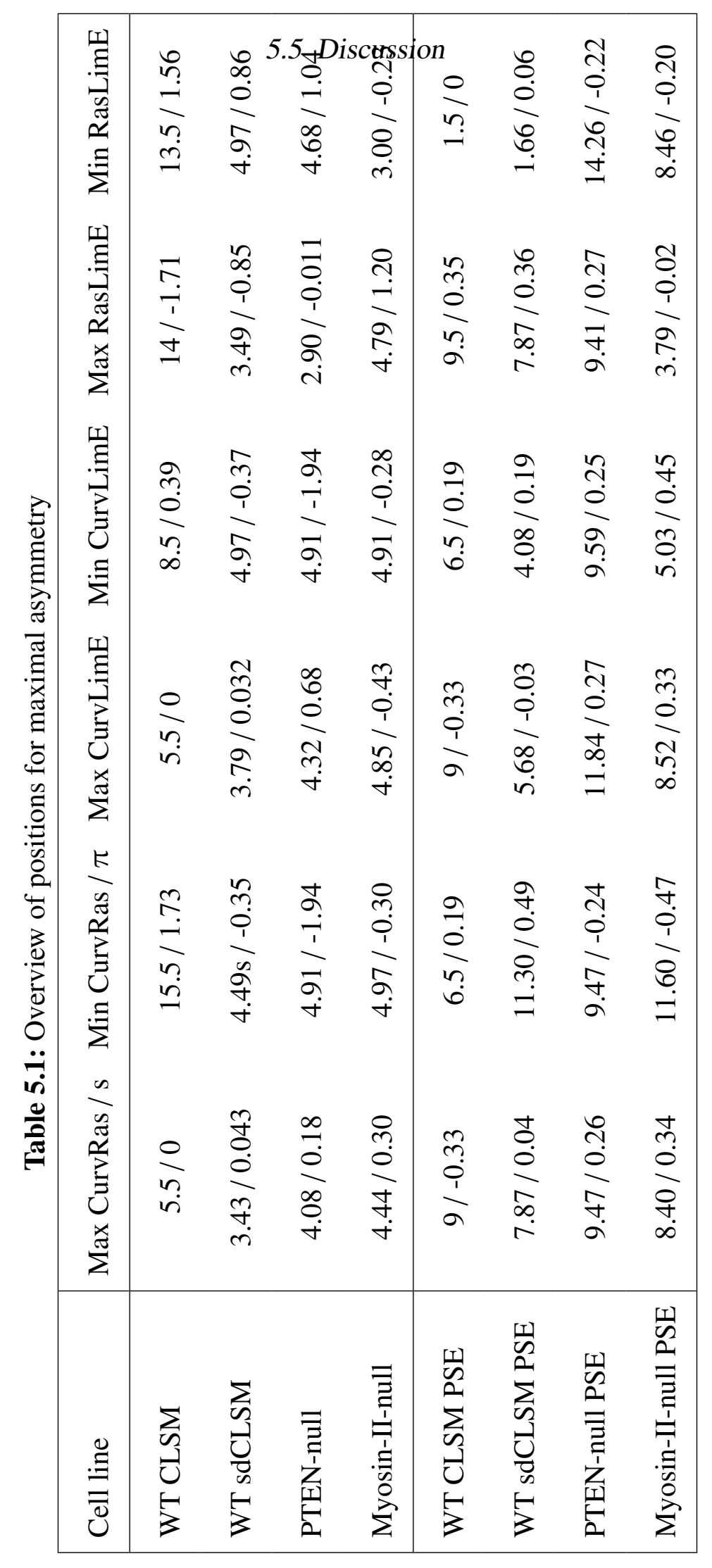




\section{CHAPTER 6}

\section{Conclusion and Outlook}

\subsection{Curvotaxis}

In the present work, I could prove that migration of $D . d$. cells is strongly dependent on the morphology of their substrate. A strong effect on curved substrates directing migration, hence called "Curvotaxis" was found while searching for a more realistic experimental setup. To get closer to the natural habitat of the amoeba, two steps regarding more complex substrate geometries were taken. The first step was to use a cylindrical optical fiber that has a non-vanishing constant curvature in one direction and zero curvature in the perpendicular direction. I found that cells show a preferred migration direction parallel to the direction of highest curvature.

The second step of increasing the complexity involved to mimic the $D . d$. cell migration on a wave-like substrate. Thereby, we also introduced areas with negative curvature. An interesting finding in this setup is that cells avoid to be curved 
positively and negatively at the same time, which would happen if they would migrate perpendicular to the wrinkles on the substrate. My hypothesis is that this way of holding the cell membrane curvature constant is one of the crucial driving forces of $D . d$. cell migration on curved substrates.

A possible way to explain the curvotaxis mechanism, is to regard the cell cortex in terms of the persistence length of filamentous actin. It denotes $10 \mu \mathrm{m}$ to $15 \mu \mathrm{m}$ as presented in literature by Salbreux et al. (66). On this length scale sensing of the curvature radii used in my experiments appears realistic. A proof of this hypothesis is still left for future work.

To further increase the complexity it would be possible to track $D$. $d$. cells migrating in real three-dimensional gels, and track them in all three dimensions. This kind of experiments have been started by (52). In this work of Mandeville and colleagues, neutrophils are migrating through a three-dimensional matrix of amniotic membrane due to a chemotactic gradient and are visualized with fluorescent labels, every $30 s-60 s$.

\subsection{Pattern Formation in the Actin Cortex}

The pattern formation processes in the actin cortex of $D . d$. cells are of uttermost importance for their migration and are conserved up to mammalian cells.

In this thesis I could show that the curvature, the activated patches of Ras-G and filamentous actin coincide well, during chemotactic cell migration. This was found in two different ways.

1) For defined patches of high fluorescence intensity of both labels, the mean value of the distribution of the curvature and as well of the respectively other fluorescent label 
increased linearly for increasing thresholds of the patch. This finding is consistent for both experimental setups (CLSM, sdCLSM) and it holds for wild type cells as well as for PTEN-null cells as it does for Myosin-II-null cells. This confirms the strong correlation, but not yet specifies any spatiotemporal dynamics.

2) To increase the understanding of the cause-and-effect relation between the three investigated signals (membrane curvature, Ras-G activity and actin polymerization), two-dimensional cross-correlation functions were derived. Thus it was proven by an anisotropy in the cross-correlation functions that the membrane curvature is following the dynamics of the chemotactic signaling cascade and the actin polymerization. Finally, my results suggest that the signaling protein Ras can be observed first. With just a small time lag we find the label for actin polymerization (LimE) and curvature follows both of those signals. Hence, I confirmed the cause-and-effect relation that was proposed earlier, and I could not find indications that the curvature of the cell membrane induces the signaling.

\subsection{Investigation of Signaling Dynamics in}

\section{Curvotactic Migration}

Even though the two projects presented in chapters 4 and 5 appear to be very separated at first glance, the upcoming steps of both projects lead into a combining of both ideas. The three-dimensional analysis of the signaling dynamics is the next step for the analysis of the pattern formation in the cortex. Doing so, I would like to investigate the active signaling patches in the whole cell using ultra-fast 3D imaging 

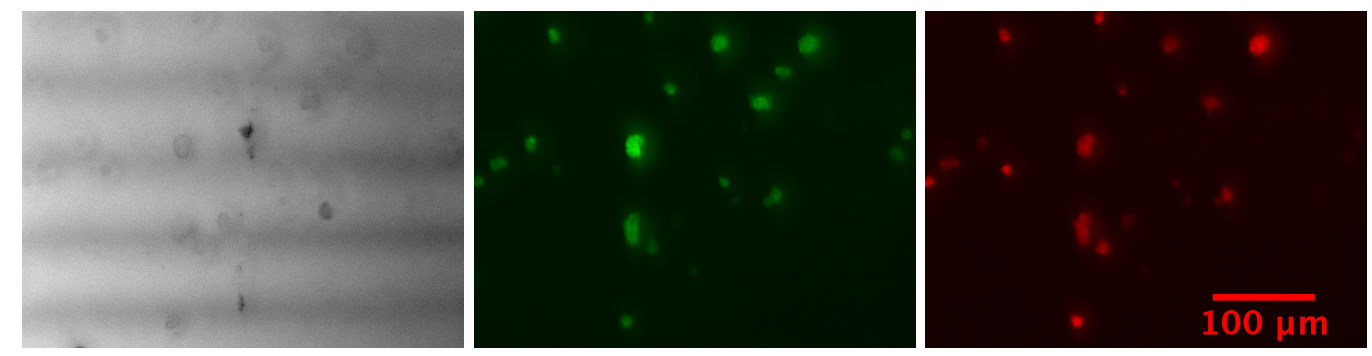

Figure 6.1: Micrographs of Myosin-II-GFP+LimE-mRFP labeled $D . d$. cells on wrinkled substrate. All images are recorded within $1.5 \mathrm{~s}$. Left: Brightfield image; the wrinkles are visible wtih the regular pattern of the black and white changes. The cells are visible. Center: Fluorescence image of GFP; Myosin-II is visualized inside the cells in green via GFP. Right: Fluorescence image of mRFP; Filamentous actin is visualized inside the cells in red via mRFP. Scale bar corresponds to $100 \mu \mathrm{m}$ in all three graphs.

with the sdCLSM, instead of individual confocal slices. The group of Danuser published work in a very similar direction recently (30). They investigated primary aortic endothelial cells in a three-dimensional collagen gel and in microfabricated agarose chambers. The investigation of the branching of the endothelial cells could be connected to cortical myosin-II association and dissociation which was found to be regulated in a negative manner by cell-surface curvature.

To include fluorescence microscopy of labeled signaling proteins in the curvotaxis investigation would help to distinguish the origin of curvotaxis and is easily possible using the wrinkled substrates, as is shown in Figure 6.1, where I present a proofof-concept image of the fluorescent imaging of wildtype $D . d$. cells labeled with Myosin-II-GFP and LimE-mRFP on the wrinkled substrate. The wrinkles are visible in the brightfield image but not in the fluorescence images. All three images are recorded within $1.5 \mathrm{~s}$. Hence it is certain that the project can progress further due to studying signaling dynamics of chemotactically and curvotactically migrating cells 
in full three dimensions.

Even though the BAR domain is unlikely to explain curvotaxis, it would be useful to investigate whether there is a connection of the BAR proteins with curvotaxis. To achieve that $D . d$. cells where a BAR protein, for instance IBAR, is tagged with a fluorescent marker could be investigated on the wrinkled substrate, as the imaging of fluorescent probes can be done easily with this setup.

A possible way to explain the curvotaxis mechanism, is to regard the cell cortex in terms of the persistence length of filamentous actin. It denotes $10 \mu \mathrm{m}$ to $15 \mu \mathrm{m}$ as presented in literature by Salbreux et al. (66). On this length scale sensing of the curvature radii used in my experiments appears realistic. A proof of this hypothesis is still left for future work.

\subsection{Outlook}

Besides the possible connection of the two fascinating subjects of this thesis, there is more further work to do. Maybe the most important and interesting question is to investigate the origin of the curvotaxis. This could involve visualization of important proteins during curvotactic migration. Another fascinating question is to ask about the interplay of curvotaxis with other directed migration mechanisms as chemotaxis or contact guidance. One could ask the question which of the three mentioned mechanisms is the strongest or "Can curvotaxis overrule chemotaxis?" To answer this question one would need a experimental setup, that allows to apply a well-controlled chemotactic gradient, to cells migrating on a cylinder. To compare curvotaxis and contact guidance, a possible way would be to construct cylinders, 
which have nanometer-sized topography along the flat axis. The line of progression to get close to a realistic experiment for curvotaxis would be to use three-dimensional gels like fibronectin-gels with different fiber radii and add a chemotactic gradient to this setup.

An important question, which should be further investigated is the interplay of Ras-G activity and actin polymerization. Are there ways to enable the $D$. $d$. cells, or other cells, which migrate in an amoeboid fashion, to migrate quicker or more efficient. As a long-term goal this could lead to improved medication. 


\section{Bibliography}

[1] Bruce Alberts, Alexander Johnson, Julian Lewis, Martin Raff, Keith Roberts, and Peter Walter. Molecular Biology of the Cell. Garland Science, 2002.

[2] Revathi Ananthakrishnan and Allen Ehrlicher. The forces behind cell movement. International Journal of Biological Sciences, 3(5):303-317, 2007.

[3] Bruno Antonny. Mechanisms of membrane curvature sensing. Annual Review of Biochemistry, 80(1):101-123, 2011. PMID: 21438688.

[4] Delphine Arcizet, Sofia Capito, Mari Gorelashvili, Carolin Leonhardt, Marion Vollmer, Simon Youssef, Susanne Rappl, and Doris Heinrich. Contact-controlled amoeboid motility induces dynamic cell trapping in $3 \mathrm{~d}-$ microstructured surfaces. Soft Matter, (5):1473-1481, 2012.

[5] Albert J Bae and Eberhard Bodenschatz. On the swimming of dictyostelium amoebae. Proc Natl Acad Sci U S A, 107(44):E165-6, Nov 2010.

[6] Christian Bär. Elementare Differentialgeometrie. Walter de Gruyter, 2001. 
[7] Christian F.W Becker, Christie L Hunter, Ralf P Seidel, Stephen B.H Kent, Roger S Goody, and Martin Engelhard. A sensitive fluorescence monitor for the detection of activated ras: total chemical synthesis of site-specifically labeled ras binding domain of c-raf1 immobilized on a surface. Chemistry $\mathcal{E}$ Biology, $8(3): 243-252,2001$.

[8] Carsten Beta, Danica Wyatt, Wouter-Jan Rappel, and Eberhard Bodenschatz. Flow photolysis for spatiotemporal stimulation of single cells. Analytical Chemistry, 79(10):3940-3944, 2007. PMID: 17432827.

[9] Eric Betzig, George H. Patterson, Rachid Sougrat, O. Wolf Lindwasser, Scott Olenych, Juan S. Bonifacino, Michael W. Davidson, Jennifer LippincottSchwartz, and Harald F. Hess. Imaging intracellular fluorescent proteins at nanometer resolution. Science, 313(5793):1642-1645, 2006.

[10] Parvin Bolourani, George B Spiegelman, and Gerald Weeks. Delineation of the roles played by rasg and rasc in camp-dependent signal transduction during the early development of dictyostelium discoideum. Molecular Biology of the Cell, 17(10):4543-4550, 102006.

[11] J. T. Bonner, A. Hay, D. G. John, and H. B. Suthers. ph affects fruiting and slug orientation in dictyostelium discoideum. Journal of Embryology and Experimental Morphology, 87(1):207-213, 1985.

[12] Leonard Bosgraaf and Peter J. M. Van Haastert. Navigation of chemotactic cells by parallel signaling to pseudopod persistence and orientation. PLOS ONE, 4(8):e6842, 082009. 
[13] Leonard Bosgraaf and Peter J. M. Van Haastert. The ordered extension of pseudopodia by amoeboid cells in the absence of external cues. PLOS ONE, 4(4):e5253, 042009.

[14] Leonard Bosgraaf and Peter J.M. van Haastert. The regulation of myosin ii in dictyostelium. European Journal of Cell Biology, 85(9-10):969 - 979, 2006. A special issue dedicated to Guenter Gerisch on the occasion of his 75th birthday.

[15] Till Bretschneider, Stefan Diez, Kurt Anderson, John Heuser, Margaret Clarke, Annette Müller-Taubenberger, Jana Köhler, and Günther Gerisch. Dynamic actin patterns and arp2/3 assembly at the substrate-attached surface of motile cells. Current Biology, 14(1):1-10, 2014/12/22 2004.

[16] E. Cerda and L. Mahadevan. Geometry and physics of wrinkling. Phys. Rev. Lett., 90:074302, Feb 2003.

[17] Alex Chattwood, Parvin Bolourani, and Gerald Weeks. Rasg signaling is important for optimal folate chemotaxis in dictyostelium. BMC Cell Biology, 15(1):13, 2014.

[18] R L Chisholm, S Hopkinson, and H F Lodish. Superinduction of the dictyostelium discoideum cell surface camp receptor by pulses of camp. Proceedings of the National Academy of Sciences of the United States of America, 84(4):1030-1034, 021987.

[19] Kristopher Clark, Michiel Langeslag, Carl G. Figdor, and Frank N. van Leeuwen. Myosin \{II $\}$ and mechanotransduction: a balancing act. Trends in Cell Biology, 17(4):178 - 186, 2007. 
[20] Dee N. Dao, Richard H. Kessin, and Herbert L. Ennis. Developmental cheating and the evolutionary biology of dictyostelium and myxococcus. Microbiology, 146(7):1505-1512, 2000.

[21] Marc A. de la Roche and Graham P. Côté. Regulation of dictyostelium myosin i and \{II \}. Biochimica et Biophysica Acta (BBA) - General Subjects, 1525(3):245 $-261,2001$.

[22] É. Debreuve, M. Gastaud, M. Barlaud, and G. Aubert. Using the shape gradient for active contour segmentation: from the continuous to the discrete formulation. Journal of Mathematical Imaging and Vision, 28(1):47-66, 2007.

[23] Emmanuel Décavé, Didier Rieu, Jérémie Dalous, Sébastien Fache, Yves Bréchet, Bertrand Fourcade, Michel Satre, and Franz Bruckert. Shear flowinduced motility of dictyostelium discoideum cells on solid substrate. Journal of Cell Science, 116(21):4331-4343, 2003.

[24] Dirk Dormann, Ji-Yun Kim, Peter N. Devreotes, and Cornelis J. Weijer. camp receptor affinity controls wave dynamics, geometry and morphogenesis in dictyostelium. Journal of Cell Science, 114(13):2513-2523, 2001.

[25] Meghan K. Driscoll, Colin McCann, Rael Kopace, Tess Homan, John T. Fourkas, Carole Parent, and Wolfgang Losert. Cell shape dynamics: From waves to migration. PLoS Comput Biol, 8(3):e1002392, 032012.

[26] Meghan K. Driscoll, Xiaoyu Sun, Can Guven, John T. Fourkas, and Wolfgang Losert. Cellular contact guidance through dynamic sensing of nanotopography. ACS Nano, 8(4):3546-3555, 2014. PMID: 24649900. 
[27] G.A. Dunn and J.P. Heath. A new hypothesis of contact guidance in tissue cells. Experimental Cell Research, 101(1):1 - 14, 1976.

[28] A F M Mar e, A V Panfilov, and P Hogeweg. Phototaxis during the slug stage of dictyostelium discoideum: a model study. Proceedings of the Royal Society B: Biological Sciences, 266(1426):1351-1351, 071999.

[29] L. Eichinger, J. A. Pachebat, G. Glockner, M. A. Rajandream, R. Sucgang, M. Berriman, J. Song, R. Olsen, K. Szafranski, Q. Xu, B. Tunggal, S. Kummerfeld, M. Madera, B. A. Konfortov, F. Rivero, A. T. Bankier, R. Lehmann, N. Hamlin, R. Davies, P. Gaudet, P. Fey, K. Pilcher, G. Chen, D. Saunders, E. Sodergren, P. Davis, A. Kerhornou, X. Nie, N. Hall, C. Anjard, L. Hemphill, N. Bason, P. Farbrother, B. Desany, E. Just, T. Morio, R. Rost, C. Churcher, J. Cooper, S. Haydock, N. van Driessche, A. Cronin, I. Goodhead, D. Muzny, T. Mourier, A. Pain, M. Lu, D. Harper, R. Lindsay, H. Hauser, K. James, M. Quiles, M. Madan Babu, T. Saito, C. Buchrieser, A. Wardroper, M. Felder, M. Thangavelu, D. Johnson, A. Knights, H. Loulseged, K. Mungall, K. Oliver, C. Price, M. A. Quail, H. Urushihara, J. Hernandez, E. Rabbinowitsch, D. Steffen, M. Sanders, J. Ma, Y. Kohara, S. Sharp, M. Simmonds, S. Spiegler, A. Tivey, S. Sugano, B. White, D. Walker, J. Woodward, T. Winckler, Y. Tanaka, G. Shaulsky, M. Schleicher, G. Weinstock, A. Rosenthal, E. C. Cox, R. L. Chisholm, R. Gibbs, W. F. Loomis, M. Platzer, R. R. Kay, J. Williams, P. H. Dear, A. A. Noegel, B. Barrell, and A. Kuspa. The genome of the social amoeba dictyostelium discoideum. Nature, 435(7038):43-57, 052005.

[30] Hunter Elliott, Robert S. Fischer, Kenneth A. Myers, Ravi A. Desai, Lin Gao, Christopher S. Chen, Robert S. Adelstein, Clare M. Waterman, and Gaudenz 
Danuser. Myosin ii controls cellular branching morphogenesis and migration in three dimensions by minimizing cell-surface curvature. Nat Cell Biol, 17(2):137-147, 022015.

[31] Petra Fey, Anthony S Kowal, Pascale Gaudet, Karen E Pilcher, and Rex L Chisholm. Protocols for growth and development of dictyostelium discoideum. Nat. Protocols, 2(6):1307-1316, 062007.

[32] Markus Fischer, Ilka Haase, Evelyn Simmeth, Günther Gerisch, and Annette Müller-Taubenberger. A brilliant monomeric red fluorescent protein to visualize cytoskeleton dynamics in dictyostelium. \{FEBS\} Letters, 577(1-2):227 - 232, 2004.

[33] Jonathan M. Flowers, Si I. Li, Angela Stathos, Gerda Saxer, Elizabeth A. Ostrowski, David C. Queller, Joan E. Strassmann, and Michael D. Purugganan. Variation, sex, and social cooperation: Molecular population genetics of the social amoeba Dictyostelium discoideum. PLoS Genet, 6(7):e1001013, 07 2010.

[34] Mari Gorelashvili, Martin Emmert, Kai F Hodeck, and Doris Heinrich. Amoeboid migration mode adaption in quasi-3d spatial density gradients of varying lattice geometry. New Journal of Physics, 16(7):075012, 2014.

[35] Inbal Hecht, Monica L. Skoge, Pascale G. Charest, Eshel Ben-Jacob, Richard A. Firtel, William F. Loomis, Herbert Levine, and Wouter-Jan Rappel. Activated membrane patches guide chemotactic cell motility. PLoS Comput Biol, 7(6):e1002044, 062011. 
[36] Stefan W. Hell and Jan Wichmann. Breaking the diffraction resolution limit by stimulated emission: stimulated-emission-depletion fluorescence microscopy. Opt. Lett., 19(11):780-782, Jun 1994.

[37] Samuel T. Hess, Thanu P.K. Girirajan, and Michael D. Mason. Ultra-high resolution imaging by fluorescence photoactivation localization microscopy. Biophysical Journal, 91(11):4258 - 4272, 2006.

[38] Choo B Hong, Donna R Fontana, and Kenneth L Poff. Thermotaxis of dictyostelium discoideum amoebae and its possible role in pseudoplasmodial thermotaxis. Proceedings of the National Academy of Sciences of the United States of America, 80(18):5646-5649, 091983.

[39] Yi Elaine Huang, Miho Iijima, Carole A Parent, Satoru Funamoto, Richard A Firtel, and Peter Devreotes. Receptor-mediated regulation of pi3ks confines pi $(3,4,5) \mathrm{p}(3)$ to the leading edge of chemotaxing cells. Molecular Biology of the Cell, 14(5):1913-1922, 052003.

[40] R Insall, A Kuspa, P J Lilly, G Shaulsky, L R Levin, W F Loomis, and P Devreotes. Crac, a cytosolic protein containing a pleckstrin homology domain, is required for receptor and g protein-mediated activation of adenylyl cyclase in dictyostelium. The Journal of Cell Biology, 126(6):1537-1545, 1994.

[41] Noo Li Jeon, Stephan K. W. Dertinger, Daniel T. Chiu, Insung S. Choi, Abraham D. Stroock, and George M. Whitesides. Generation of solution and surface gradients using microfluidic systems. Langmuir, 16(22):8311-8316, 2000. 
[42] Helmut Kae, Chinten James Lim, George B Spiegelman, and Gerald Weeks. Chemoattractant-induced ras activation during dictyostelium aggregation. EMBO reports, 5(6):602-606, 2004.

[43] Rama Kataria, Xuehua Xu, Fabrizia Fusetti, Ineke Keizer-Gunnink, Tian Jin, Peter J. M. van Haastert, and Arjan Kortholt. Dictyostelium ric8 is a nonreceptor guanine exchange factor for heterotrimeric $\mathrm{g}$ proteins and is important for development and chemotaxis. Proceedings of the National Academy of Sciences, 110(16):6424-6429, 2013.

[44] Richard H. Kessin. Dyctostelium. Cambridge University Press, 2001.

[45] J.Y. Kim, J.A. Borleis, and P.N. Devreotes. Switching of chemoattractant receptors programs development and morphogenesis in dictyostelium: Receptor subtypes activate common responses at different agonist concentrations. Developmental Biology, 197(1):117 - 128, 1998.

[46] PS Klein, TJ Sun, CL Saxe, AR Kimmel, RL Johnson, and PN Devreotes. A chemoattractant receptor controls development in dictyostelium discoideum. Science, 241(4872):1467-1472, 1988.

[47] Arjan Kortholt, Parvin Bolourani, Holger Rehmann, Ineke Keizer-Gunnink, Gerald Weeks, Alfred Wittinghofer, and Peter J.M. Van Haastert. A rap/phosphatidylinositol 3-kinase pathway controls pseudopod formation. Molecular Biology of the Cell, 21(6):936-945, 2010.

[48] Arjan Kortholt, Rama Kataria, Ineke Keizer-Gunnink, Wouter N Van Egmond, Ankita Khanna, and Peter J M Van Haastert. Dictyostelium chemotaxis: essen- 
tial ras activation and accessory signalling pathways for amplification. $E M B O$ reports, 12(12):1273-1279, 2011.

[49] Arjan Kortholt, Ineke Keizer-Gunnink, Rama Kataria, and Peter J. M. Van Haastert. Ras activation and symmetry breaking during dictyostelium chemotaxis. Journal of Cell Science, 126(19):4502-4513, 2013.

[50] Arjan Kortholt and Peter J.M. van Haastert. Highlighting the role of ras and rap during dictyostelium chemotaxis. Cellular Signalling, 20(8):1415 - 1422, 2008.

[51] Joern Linkner, Gregor Witte, Hongxia Zhao, Alexander Junemann, Benjamin Nordholz, Petra Runge-Wollmann, Pekka Lappalainen, and Jan Faix. The inverse bar domain protein ibara drives membrane remodeling to control osmoregulation, phagocytosis and cytokinesis. Journal of Cell Science, 127(6):1279$1292,2014$.

[52] J T Mandeville, M A Lawson, and F R Maxfield. Dynamic imaging of neutrophil migration in three dimensions: mechanical interactions between cells and matrix. Journal of Leukocyte Biology, 61(2):188-200, 1997.

[53] C.R. McCudden, M.D. Hains, R.J. Kimple, D.P. Siderovski, and F.S. Willard. G-protein signaling: back to the future. Cellular and Molecular Life Sciences, 62(5):551-577, 2005.

[54] Kota Miura. Phototaxis of Dictyostelium discoideum Slugs. PhD thesis, September 2000 .

[55] Oliver Nagel, Can Guven, Matthias Theves, Meghan Driscoll, Wolfgang Losert, 
and Carsten Beta. Geometry-driven polarity in motile amoeboid cells. PLoS ONE, 9(12):e113382, 122014.

[56] A.A. Noegel and M. Schleicher. The actin cytoskeleton of dictyostelium: a story told by mutants. Journal of Cell Science, 113(5):759-766, 2000.

[57] Danton H. O'Day and Alex Keszei. Signalling and sex in the social amoebozoans. Biological Reviews, 87(2):313-329, 2012.

[58] T.D. Pollard, W.C. Earnshaw, and J. Lippincott-Schwartz. Cell Biology. Elsevier Health Sciences, 2007.

[59] Thomas D. Pollard. The cytoskeleton, cellular motility and the reductionist agenda. Nature, 422(6933):741-745, 042003.

[60] Thomas D. Pollard. Regulation of actin filament assembly by arp2/3 complex and formins. Annual Review of Biophysics and Biomolecular Structure, 36(1):451-477, 2007. PMID: 17477841.

[61] Thomas D. Pollard and John A. Cooper. Actin, a central player in cell shape and movement. Science, 326(5957):1208-1212, 2009.

[62] Paolo P Provenzano, David R Inman, Kevin W Eliceiri, Steven M Trier, and Patricia J Keely. Contact guidance mediated three-dimensional cell migration is regulated by rho/rock-dependent matrix reorganization. Biophysical Journal, 95(11):5374-5384, 122008.

[63] YuA Rovensky and V.I. Samoilov. Morphogenetic response of cultured normal and transformed fibroblasts, and epitheliocytes, to a cylindrical substratum 
surface. possible role for the actin filament bundle pattern. Journal of Cell Science, 107(5):1255-1263, 1994.

[64] Michael J Rust, Mark Bates, and Xiaowei Zhuang. Sub-diffraction-limit imaging by stochastic optical reconstruction microscopy (storm). Nat Meth, 3(10):793-796, 102006.

[65] Juha Saarikangas, Hongxia Zhao, Anette Pykäläinen, Pasi Laurinmäki, Pieta K. Mattila, Paavo K. J. Kinnunen, Sarah J. Butcher, and Pekka Lappalainen. Molecular mechanisms of membrane deformation by i-bar domain proteins. Current Biology, 19(2):95-107, 2015/07/13.

[66] Guillaume Salbreux, Guillaume Charras, and Ewa Paluch. Actin cortex mechanics and cellular morphogenesis. Trends in Cell Biology, 22(10):536 - 545, 2012.

[67] Atsuo T. Sasaki, Cheryl Chun, Kosuke Takeda, and Richard A. Firtel. Localized ras signaling at the leading edge regulates pi3k, cell polarity, and directional cell movement. The Journal of Cell Biology, 167(3):505-518, 2004.

[68] Lynne J. Shanley, Petr Walczysko, Mary Bain, David J. MacEwan, and Min Zhao. Influx of extracellular ca2+ is necessary for electrotaxis in dictyostelium. Journal of Cell Science, 119(22):4741-4748, 2006.

[69] Monica Skoge, Haicen Yue, Michael Erickstad, Albert Bae, Herbert Levine, Alex Groisman, William F. Loomis, and Wouter-Jan Rappel. Cellular memory in eukaryotic chemotaxis. Proceedings of the National Academy of Sciences, 111(40):14448-14453, 2014. 
[70] L Song, E J Hennink, I T Young, and H J Tanke. Photobleaching kinetics of fluorescein in quantitative fluorescence microscopy. Biophysical Journal, 68(6):2588-2600, 061995.

[71] Loling Song, Sharvari M. Nadkarni, Hendrik U. Bödeker, Carsten Beta, Albert Bae, Carl Franck, Wouter-Jan Rappel, William F. Loomis, and Eberhard Bodenschatz. Dictyostelium discoideum chemotaxis: Threshold for directed motion. European Journal of Cell Biology, 85(9-10):981 - 989, 2006. A special issue dedicated to Guenter Gerisch on the occasion of his 75th birthday.

[72] David J. Stephens and Victoria J. Allan. Light microscopy techniques for live cell imaging. Science, 300(5616):82-86, 2003.

[73] Kristen F. Swaney, Chuan-Hsiang Huang, and Peter N. Devreotes. Eukaryotic chemotaxis: A network of signaling pathways controls motility, directional sensing, and polarity. Annual Review of Biophysics, 39(1):265-289, 2010. PMID: 20192768.

[74] Stephen P. Szabo, Danton H. O'Day, and Abdul H. Chagla. Cell fusion, nuclear fusion, and zygote differentiation during sexual development of dictyostelium discoideum. Developmental Biology, 90(2):375 - 382, 1982.

[75] Kosuke Takeda, Danying Shao, Micha Adler, Pascale G. Charest, William F. Loomis, Herbert Levine, Alex Groisman, Wouter-Jan Rappel, and Richard A. Firtel. Incoherent feedforward control governs adaptation of activated ras in a eukaryotic chemotaxis pathway. Science Signaling, 5(205):ra2-ra2, 2012.

[76] Takeo Tanaami, Shinya Otsuki, Nobuhiro Tomosada, Yasuhito Kosugi, Mizuho Shimizu, and Hideyuki Ishida. High-speed 1-frame/ms scanning confocal 
microscope with a microlens and nipkow disks. Appl. Opt., 41(22):4704-4708, Aug 2002.

[77] Matthias Theves. Quantitative study of eukariotic chemotaxis with microfluidic devices. Master's thesis, Georg-August Univerität Göttingen and Max-Planck Institut für Dynamik und Selbstorganisation, 2009.

[78] AM TURING. The chemical basis of morphogenesis. Philosophical Transactions of the Royal Society of London Series B-Biological Sciences, 237:37-72, 1952.

[79] Luke Tweedy, Born Meier, Jurgen Stephan, Doris Heinrich, and Robert G. Endres. Distinct cell shapes determine accurate chemotaxis. Sci. Rep., 3, 09 2013.

[80] Richard A. Tyson, Evgeny Zatulovskiy, Robert R. Kay, and Till Bretschneider. How blebs and pseudopods cooperate during chemotaxis. Proceedings of the National Academy of Sciences, 111(32):11703-11708, 2014.

[81] B. Utter, R. Ragnarsson, and E. Bodenschatz. Alternating tip splitting in directional solidification. Phys. Rev. Lett., 86:4604-4607, May 2001.

[82] Peter J. M. Van Haastert. Chemotaxis: insights from the extending pseudopod. Journal of Cell Science, 123(18):3031-3037, 092010.

[83] Peter J. M. Van Haastert. A stochastic model for chemotaxis based on the ordered extension of pseudopods. Biophysical Journal, 99(10):3345-3354, 2015/01/112010. 
[84] Peter J.M. van Haastert, Ineke Keizer-Gunnink, and Arjan Kortholt. Essential role of pi3-kinase and phospholipase a2 in dictyostelium discoideum chemotaxis. The Journal of Cell Biology, 177(5):809-816, 2007.

[85] Douwe M. Veltman, Gunkut Akar, Leonard Bosgraaf, and Peter J.M. Van Haastert. A new set of small, extrachromosomal expression vectors for dictyostelium discoideum. Plasmid, 61(2):110 - 118, 2009.

[86] Miguel Vicente-Manzanares, Xuefei Ma, Robert S. Adelstein, and Alan Rick Horwitz. Non-muscle myosin ii takes centre stage in cell adhesion and migration. Nat Rev Mol Cell Biol, 10(11):778-790, 112009.

[87] D. J. Watts and J. M. Ashworth. Growth of myxamoebae of the cellular slime mould dictyostelium discoideum in axenic culture. Biochemical Journal, 119(2):171-174, 091970.

[88] Danica Wyatt. CHEMOTAXIS IN MICROFLUID CHANNELS. PhD thesis, Cornell University, 2007.

[89] Younan Xia and George M. Whitesides. Soft lithography. Angewandte Chemie, International Edition, 37(5):550-575, 1998.

[90] Yuan Xiong, Chuan-Hsiang Huang, Pablo A Iglesias, and Peter N Devreotes. Cells navigate with a local-excitation, global-inhibition-biased excitable network. Proc Natl Acad Sci U S A, 107(40):17079-86, Oct 2010.

[91] Sheng Zhang, Pascale G. Charest, and Richard A. Firtel. Spatiotemporal regulation of ras activity provides directional sensing. Current Biology, 18(20):1587 1593, 2008. 


\section{List of Figures}

$2.1 \quad$ Overview of the three parts of the life cycle of $D . d . . \ldots \ldots \ldots$

2.2 Main properties of actin. . . . . . . . . . . . . . 17

$2.3 \quad$ Sketch of the actin machinery. . . . . . . . . . . . . . . 19

2.4 Sketch of myosin-II and its function. . . . . . . . . . . . . 21

2.5 Migration mechanism of an amoeboid cell. . . . . . . . . . 23

2.6 Overview of most important modules in $D . d$. chemotactic signaling cascade. . . . . . . . . . . . . . . . . 26

2.7 Sketch of transduction of cAMP-signal to Ras-GEF. . . . . . . . . . 27

$2.8 \quad$ Sketch of transduction of signal from $G \alpha$ to actin polymerization via Ras. . . . . . . . . . . . . . . . . . . 29

2.9 Sketch of signal transduction from RasG until actin polymerization via PIP3. . . . . . . . . . . . . . . . 30

2.10 Sketch of chemotaxis in $D . d . . \ldots \ldots \ldots$

$3.1 \quad$ Basic idea of soft lithography. . . . . . . . . . . . . . . 41 
3.2 This sketch shows the lightpath of a laser scanning confocal microscope. . . . . . . . . . . . . . . . . . . 44

3.3 Sketch of Spinning Disc operation mode. . . . . . . . . . . . . 46

3.4 Sketch of DIC light path. . . . . . . . . . . . . . 47

3.5 Sketch of the curvotaxis setup. . . . . . . . . . . . . . . 49

3.6 Photograph of perfusion chamber. . . . . . . . . . . 50

3.7 Sketch of the construction method of the wrinkled Curvotaxis device. 51

3.8 Sketch of the gradient mixer. . . . . . . . . . . . . 52

$4.1 \quad$ Sketch of a planar curve $c$ with its first derivative. . . . . . . . . . 58

4.2 Using two-dimensional projection as measure for three-dimensional morphology. . . . . . . . . . . . . . . . . . . . . . . . 59

4.3 Sketch of projection from three-dimensional fiber to two-dimensional microscope image. . . . . . . . . . . . . . . 61

4.4 Sketch of three different exemplary trajectories with identical $d_{\text {projection. }} 63$

$4.5 \quad$ Sketch of a migrating cell and its trajectory in direction perpendicular to the wrinkles. . . . . . . . . . . . . . . . 66

4.6 Sketch of convex hull of trajectory . . . . . . . . . . . . 68

4.7 Experimental results of AFM measurements. . . . . . . . . . 70

4.8 Height profile of a single wrinkle wave. . . . . . . . . . . . 71

4.9 Overlay of the height profile (black and white inlay) and the top view bright field image. . . . . . . . . . . . . . . . . . 72

4.10 Overlay of the first micrograph of a time series of migrating cells and the corresponding 43 trajectories extracted from this series. . . 73 
4.11 Histogram of migration angles of 42 motile $D . d$. cells on the fiber shown in Figure 4.10 for all steps of the trajectories that migrate a minimum distance of $10 \mu \mathrm{m}$. A very distinct peak at a migration direction of $90^{\circ}$ is found. . . . . . . . . . . . . . . 74

4.12 Histogram showing the orientation of $D . d$. cell trajectories on an optical fiber. . . . . . . . . . . . . . . . . . 75

4.13 This plot shows the migration velocity of $D . d$. cells dependent on the migration direction of the cells for the exemplary microfluidic experiment. ...................... 77

4.14 Migration angle histogram for all experimental setups. . . . . . . . 79

4.15 Histogram showing the orientation of $D . d$. cell trajectories on optical fibers of all four different experimental setups with fiber radius $r=80 \mu \mathrm{m} . \ldots \ldots \ldots \ldots \ldots$. . . . . . . . . 80

4.16 Angle dependence of cell migration velocity of all different experimental setups. . . . . . . . . . . . . . . . . 81

4.17 Velocities $v_{\perp}, v_{\|}$, and $v_{\text {total }}$ for all four experimental setups. . . . . 83

4.18 For the different experimental setups the values of $C A P$ differ. In no-flow conditions the anisotropy is most pronounced. . . . . . . . . 84

4.19 Plot of the Curvotactic Anisotropy Parameter $(C A P)$ against the fiber radii of optical fibers. . . . . . . . . . . . . . . 86

4.20 Mean migration velocity of cells before and after chemical inhibition and knockout mutants. . . . . . . . . . . . . . . 87

$4.21 \quad$ D. $d$. cells migrating on polystyrene wrinkles. . . . . . . . . . . 89 
4.22 Histograms of migration angles on wrinkles with $r_{\max }^{c}=85.8 \mu \mathrm{m}$

for all cells and divivded in migrating steps for cells in negatively respectively positively curved regions. . . . . . . . . . . . 90

4.23 Histogram showing the orientation of $D . d$. cell trajectories on a

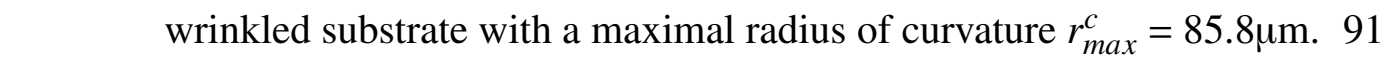

4.24 Cell orientation and direction dependence of velocity . . . . . . . . 92

4.25 Histogram of migration angle for motile cells for all different wrinkled radii. . . . . . . . . . . . . . . . . . . . . . . . . . . . . 94

4.26 Histogram showing the orientation of $D . d$. cell trajectories on all wrinkled substrates. . . . . . . . . . . . . . . . . 95

4.27 Histogram of the cell orientation angle of all different wrinkled substrate . . . . . . . . . . . . . . . 96

4.28 This plot shows the migration velocity of $D . d$. cells dependent on the migration direction of the cells for the cells migrating on all wrinkles substrates. The velocity shows no directional dependence. 96

4.29 Plot of the Curvotactic Anisotropy Parameter $(C A P)$ against the radii of maximal curvature of the wrinkled substrates. . . . . . . . . . 97

4.30 The left hand graph shows orientation and elongation of cells migrating on a wrinkled substrate. . . . . . . . . . . . . . . 98

$4.31 \quad D . d$. cell probing the direction to take on wrinkled substrate. . . . . 100

4.32 Plot of the Curvotactic Anisotropy Parameter $(C A P)$ against the radii of maximal curvature of the wrinkled substrates or fiber radius. . . . 102

5.1 Zoom on real raw data to visualise the pixel at cell membrane. . . 108

5.2 Sketch of the kymograph construction. . . . . . . . . . . . . . 109 
5.3 Sketch of principle of two-dimensional cross-correlation. . . . . . . 111

5.4 A single cell showing a pseudopod splitting. . . . . . . . . . . . . 114

5.5 Kymographs of Curvature, Ras-Fluorescence and LimE-Fluorescence 116

5.6 Dependency on the threshold of the patch definition. . . . . . 120

5.7 Distributions of curvature. . . . . . . . . . . . . . . . . . 121

5.8 Mean cross-correlations for 31 migrating cells. . . . . . . . . . . . 124

5.9 Cumulated cross-correlation function between Curvature and Ras and LimE fluorescence of 121 Pseudopods. . . . . . . . . . . . . 126

5.10 Curvature and Ras kymographs of two different WT cells with sdCLSM and CLSM. . . . . . . . . . . . . . . . 130

5.11 Dependency on the threshold of the patch definition at sdCLSM. . . 133

5.12 Cross-correlations of kymographs of 46 cells. . . . . . . . . . . . . 134

5.13 Cumulated Crosscorrelation function between Curvature and Ras and LimE fluorescence of 33 Pseudopods. . . . . . . . . . . . . 137

5.14 Dependency on the threshold of the patch definition for PTEN-null cells. . . . . . . . . . . . . . . . . . . 141

5.15 Cross-correlations of 29 kymographs extracted from 11 PTEN-null cells. . . . . . . . . . . . . . . . . . . 142

5.16 Cross-correlations of one pseudopod extracted from 11 PTEN-null cells. . . . . . . . . . . . . . . . . . . 143

5.17 Cross-correlations of 24 kymographs extracted from 16 Myosin-IInull cells. . . . . . . . . . . . . . . . . . . . . 145

5.18 Cumulated Crosscorrelation function between Curvature and Ras and LimE fluorescence of 3 pseudopod splits of Myosin-II-null cells. 147

5.19 Overview of asymmetry of cross correlation functions. . . . . . . 150 
$6.1 \quad$ Micrographs of Myosin-II-GFP+LimE-mRFP labeled $D . d$. cells on wrinkled substrate . . . . . . . . . . . . . 156 


\section{Acknowledgments}

To finish this thesis I received assistance of several people. First I want to thank Prof.

Dr. Eberhard Bodenschatz for his advice in the past four years and the possibility for me, as a theoretical physicist/mathematician, to get involved in the fascinating field of experimental biophysics and helped me to slowly develop into an experimentalist. An important person for this transformation was Dr. Marco Tarantola, who helped me a lot with the experimental aspects of the work, to understand both, the microscopy techniques and the handling of $D . d$. cells. This help combined with friendship and sharing an office for three years made Marco become the "Lord Hand" for this thesis. I like to thank Dr. Christian Westendorf for his help with the smaller and bigger problems and questions regarding the microscopes and regarding Dicties. José Negrete Jr., Hsin-Fang Hsu, Kaumudi Prabhakara, Laura Turco and Nadine Höppner have shared the experience of graduating with me, thank you for several experiments, plenty of discussions and excursions and conferences we had together. During my thesis I profited from the help of group members and visting scientist, namely Brian Utter, Albert Bae, Vladimir Zykov, Azam Gholami, Isabella Guido, Oliver 
Steinbock and Yong Wang. I also had the the pleasure to supervise the motivated student assistant and soon to be Bachelor student Nick Scholand. Without the help from our technical assistants in the cell lab life would be less easy and research would be much more difficult. Thank you to Tina Althaus, Maren Stella Müller, Marion Kunze and most of all a big THANK YOU to Katharina Schneider.

The Laboratory for Fluid Dynamics, Pattern Formation and Biocomplexity was a very nice place to graduate as all the members of the group are very nice, very helpful and very competent. I want to thank the whole team, including Angela Meister, Sabrina Volkmar, Gerhard Nolte, Andreas Kopp, Andreas Renner and Artur Kubitzek and various people of the turbulence group. Three special people of the turbulence group have to be mentioned separately: Jennifer Jucha, Michael Sinhuber and Fabio Di Lorenzo are not only colleagues but friends and helped me on a work related level, when I needed another point-of-view, and as well as personally and especially to spend hours without thinking about work.

I want to thank the members of my thesis committee Prof. Dr. Jörg Enderlein and Prof. Dr. Annette Zippelius and the members of the examination board Prof. Stephan Herminghaus and Dr. Michael Wilczek.

For the fruitful collaboration I want to thank Marius Priebe and Prof. Dr. Tim Salditt from the Institute of X-Ray Physics of the University of Göttingen, Anja Huss and Prof. Dr. Jörg Enderlein from the III. Institute of Physics of the University of Göttingen and Dr. Marco Rivetti, Dr. Oliver Bäumchen and Prof. Dr. Stephan Herminghaus from the Department of Complex Fluids of the Max Planck Institute for Dynamics and Self-Organization.

I want to thank DFG SFB 937 - "Collective behavior of soft and biological matter" for financial support. 
Additionally I want to thank the PBCS office for a lot of advice and unbureaucratic help, thanks to Michaela, Frauke, Tina and Antje.

Finally I want to thank my family for the support. Thanks to Till, who helped me improving my English skills. Last but most important I like to thank Freya and Mathis, the two most important persons in my life for everything and most for being with me. 
Acknowledgments 


\section{Curriculum Vitae}

\section{Personal Information}

Name Christoph Blum

Date of birth $\quad$ May 15, 1986

Nationality German

\section{Academic Background}

05/2011-dato Doctoral studies in Physics

Max Planck Institute for Dynamics and Self-Organization, Göttingen

Georg-August-Universität, Göttingen

International Max Planck Research School for Physics of Bio-

logical and Complex Systems (IMPRS PBC)

04/2008-04/2011 Diploma studies in Mathematics and Physics

Westfälische Wilhelms Universität, Münster 
10/2005-03/2008 Diploma studies in Mathematics and Physics

Technische Universität Clausthal.

$07 / 2005$

Abitur

Gymnasium Osterode, Osterode

\section{Publications}

M. Priebe, M. Bernhardt, C. Blum, M. Tarantola, E. Bodenschatz, and T. Salditt. Scanning X-Ray Nanodiffraction on D. $d$. . Biophys. J. 107, 2662-2673, 2014.

\section{Awards and Grants}

$02 / 2013$ Travel grant from the IMPRS PBC for the Geilo School, Soft Matter Confinement: From Biology to Physics, Geilo

$06 / 2012$ Travel grant from the IMPRS PBC for the Summer school SOFTFLOW 2012 Biological Complex Fluids, Cargèse

07/2005

School award e-fellows.net

\section{Teaching Experience}

10/2012-dato Lecturer, Georg-August-Universität, Göttingen

$\diamond$ Seminar "Pattern Formation in Physics and Biology" 
08/2011-10/2012 Teaching assistant, Georg-August-Universität, Göttingen

$\diamond$ Thermodynamics and statistical mechanics

$\diamond$ Biophysics and Complex Systems

10/2009-07/2010 Teaching assistant, Westfälische Wilhelms Universität, Münster

$\diamond$ Numerics I

$\diamond$ Numerics II

10/2007-03/2008 Teaching assistant, Technische Universität Clausthal, ClausthalZellerfeld

$\diamond$ Stochastics I

\section{Talks and Conferences}

09/2014 Dynamic Days Europe 2014, Bayreuth

Talk: "Amoeboid cell motion in complex geometry or 'Curvotaxis"'

547. WE-Heraeus-Seminar - Physics and Biology of Directed Movements of Cells and Organisms, Bad Honnef

Poster: "Dynamics and pattern formation in the actin cortex of D. d."

03/2013

Geilo School 2013 - Soft Matter Confinement: From Biology to Physics, Geilo

Poster: "Correlating signaling cascade with movement in $D . d$." 
physik, Hahnenklee

Talk: "Correlating signaling cascade with movement in $D . d . "$

$08 / 2012$

DICTY 2012 Annual International Dictyostelium Conference, Madrid

Poster: "Correlation of Ras with pseudopod fromation in $D . d . "$

$07 / 2012$

SOFTFLOW 2012 Biological Complex Fluids, Cargèse

Talk: "Pattern formation and contact angle dynamics in $D . d . "$

02/2012 Sechzehntes Harzseminar - Strukturbildung in Chemie und Biophysik, Hahnenklee

Talk: "Pattern Formation in the actin cortex of motile cells"

$11 / 2011$

JÜLICH SOFT MATTER DAYS 2011, Bonn

Poster: "Pattern Formation and Instabilities in the Actin Cortex of Motile Cells"

Göttingen, August 6, 2015 